\title{
Exploring the Potential Use of Telehealth Technology to Improve the Diagnostic Process of Autism Spectrum Disorder (ASD) in Wales, United Kingdom
}

\author{
Manahil Mohammed Alfuraydan \\ Submitted to Swansea University in fulfilment of the requirements for the \\ Degree of Doctor of Philosophy
}

Swansea University

2020

Copyright: The author, Manahil M. Alfuraydan, 2021. 


\begin{abstract}
There is a significant delay between parents initially seeking help and receiving a diagnosis of Autism Spectrum Disorder (ASD). Telehealth has the potential to accelerate the ASD diagnostic pathway. However, user acceptance is essential for the successful implementation of telehealth technology. This thesis therefore aims to examine whether telehealth can be used to increase access to ASD diagnostic services in Wales and reduce the time taken to receive a diagnosis. To achieve this, a multimethod approach was adopted, comprising of three studies. Initially, a scoping review (Study1) was conducted examining the use of telehealth in ASD diagnostic assessment. This identified two methods: (a) Real-Time (b) Store-and-Forward, particularly the Naturalistic Observation Diagnostic Assessment (NODA) system. Results also suggest that telehealth is feasible and acceptable to the families. However, findings indicate that no study had thus far examined the factors of telehealth acceptance, based on theoretical foundation. Therefore, Studies two and three were conducted, to explore the parental experience of the diagnostic process, and investigate the predictors of their acceptance to use NODA for ASD diagnostic assessment, using the Unified Theory of Acceptance and Use of Technology model. Data for both studies was collected via an online questionnaire, targeting parents of children with ASD in Wales. Descriptive and inferential statistics were applied to the closed-ended questions, and thematic analysis was used for openended questions. Study 2 findings revealed that the majority of parents were dissatisfied with the diagnostic process, with the time taken to obtain a diagnosis being a predictor of their satisfaction. Study 3 found that the majority of parents felt positively towards the NODA, with their willingness to use the system being predicted by their age, performance expectancy, social influence and comfort with using laptop and smartphone devices. Implications and recommendations are further discussed at the end of the thesis.
\end{abstract}




\section{Declarations and Statements}

\section{DECLARATION}

This work has not previously been accepted in substance for any degree and is not being concurrently submitted in candidature for any degree.

Signed

Date $. .16 / 12 / 2020$

\section{STATEMENT 1}

This thesis is the result of my own investigations, except where otherwise stated. Where correction services have been used, the extent and nature of the correction is clearly marked in a footnote(s).

Other sources are acknowledged by footnotes giving explicit references. A bibliography is appended.

Signed

Date $.16 / 12 / 2020$

\section{STATEMENT 2}

I hereby give consent for my thesis, if accepted, to be available for photocopying and for inter-library loan, and for the title and summary to be made available to outside organisations.

Signed

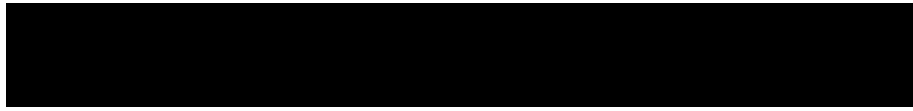

Date $16 / 12 / 2020$ 


\section{Acknowledgements}

This work would not have been possible without all the help and support I have received over several years. I would like to firstly take this opportunity to express my sincere gratitude to my supervisory team, Dr Jodie Croxall and Professor Sinead Brophy, for their insightful and invaluable contribution to my work. This $\mathrm{PhD}$ study would not have been possible without their unfailing support, guidance and encouragement throughout. Next, I am extremely grateful to all staff at the Swansea University Medical School for their considerate guidance at all times. I would like to extend a special thanks to all the parents of children with Autism Spectrum Disorder (ASD) in Wales, who took part in this study and made this research possible. I also would like to give great thanks to my parents Mohammed Alfuraydan and Ebtisam Alyaqoot, my brothers and sisters, who were a constant source of encouragement, in spite of the fact that I was studying far from home, in the UK. Their words never failed to move me and always served to strengthen my resolve to meet my $\mathrm{PhD}$ goal. Lastly, I would like to express my most warm thanks to my husband Abdullah Alkamis, and my daughters Lamar, Dalia and Tala who accompanied me on my $\mathrm{PhD}$ journeywithout their unconditional love, support and patience I could not have achieved this.

Many thanks to you all.

Manahil Alfurydan 


\section{List of Publications and Presentations}

Alfuraydan, M., Croxall, J., Hurt, L., Kerr, M., \& Brophy, S. (2020). Use of Telehealth for Facilitating the Diagnostic Assessment of Autism Spectrum Disorder (ASD): A Scoping Review. PloS one, 15(7), 1-16. (see Appendix 1).

Alfuraydan, M., Croxall, J., \& Brophy, S. (2018). Exploring the Potential Use of Telehealth to Improve the Diagnostic Process of Autism Spectrum Disorder (ASD) in Swansea, UK. Poster presented at the $13^{\text {th }}$ World Congress on Healthcare \& Technologies, Dublin, Ireland.

Alfuraydan, M. (2017). Bridging the Gap: Use of Telehealth to Support the Needs of Autistic People. Poster presented at the Postgraduate Research Students Annual Conference, Swansea, UK. 


\section{Table of Contents}

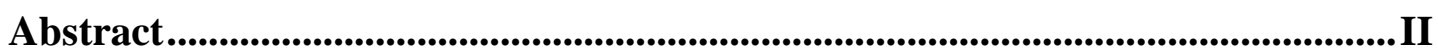

Declarations and Statements............................................................................... III

Acknowledgements...........................................................................................................................IV

List of Publications and Presentations ..........................................................

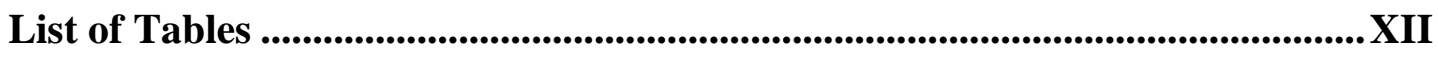

List of Figures ......................................................................................................................XIV

Abbreviations ......................................................................................................XVI

Chapter 1: Introduction .............................................................................................................1 1

1.1 Introduction ............................................................................................................................1 1

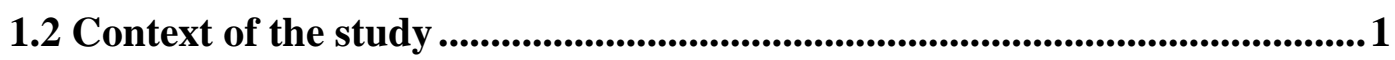

1.3 Study setting and population ...............................................................4

1.4 Statement of the problem ...........................................................................5

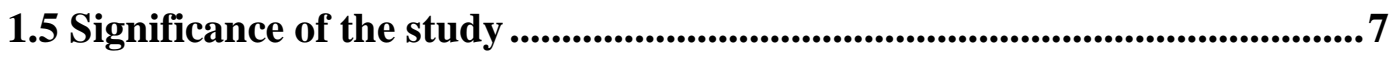

1.6 Aim and objectives ..................................................................................11

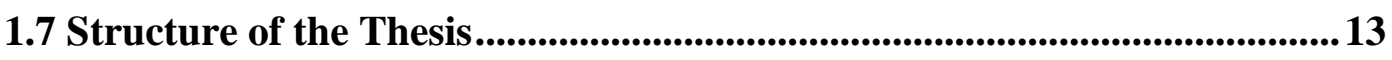

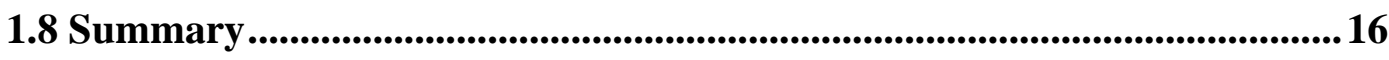

Chapter 2: Contributing Research Fields into ASD Diagnosis and Telehealth .17

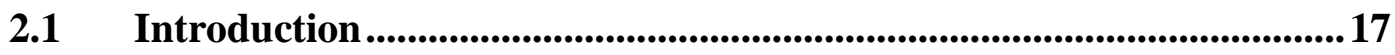

2.2 Definitions and diagnostic criteria of ASD .........................................17

2.3 Co-occurring conditions .............................................................................19

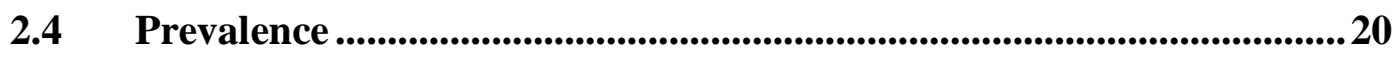

2.5 Diagnostic process of ASD in the UK ................................................21

2.6 Diagnostic process of ASD in Wales ....................................................22

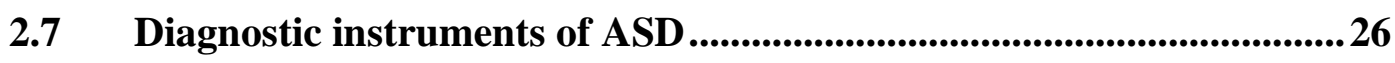

2.8 Difficulties in obtaining a diagnosis....................................................28

2.9 Parental satisfaction with the experience of receiving an ASD diagnosis and associated factors 
2.10 Telehealth definitions................................................................................38

2.11 Communication technologies .....................................................................40

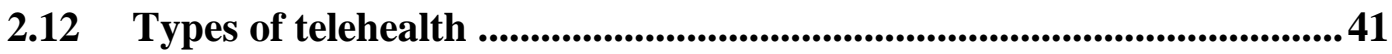

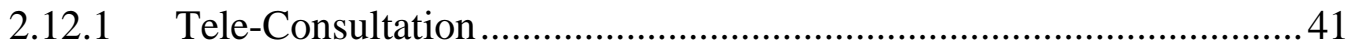

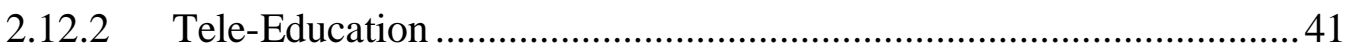

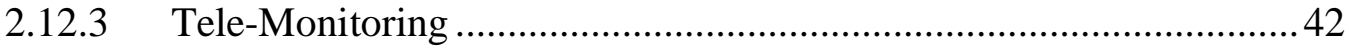

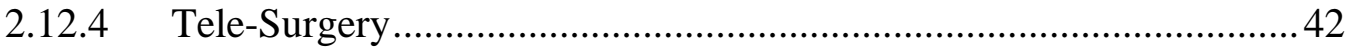

2.13 Use of telehealth with clinical specialties and ASD condition .............42

2.13.1 Use of telehealth within clinical specialties ...................................42

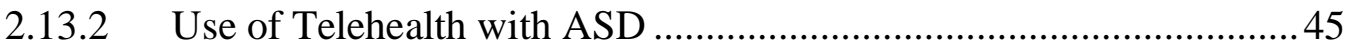

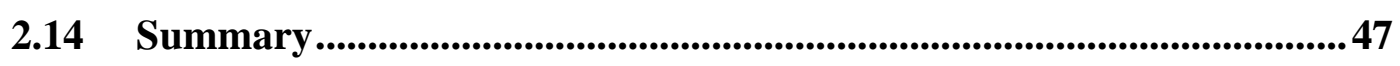

Chapter 3: (Study1) Use of Telehealth for Facilitating the Diagnostic Assessment of Autism Spectrum Disorder (ASD): A Scoping Review.................49

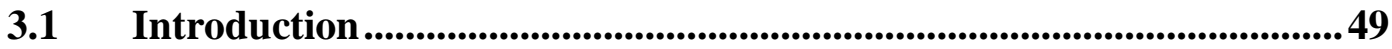

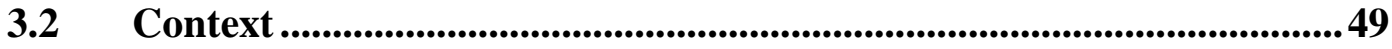

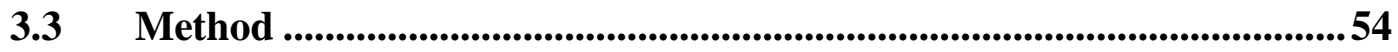

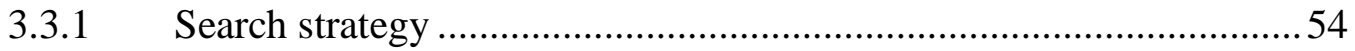

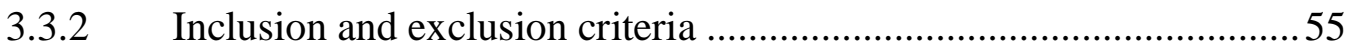

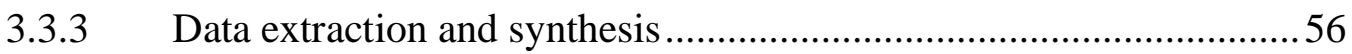

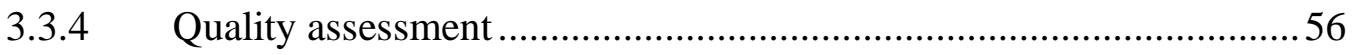

3.3.5 Publication and roles of the authors:................................................57

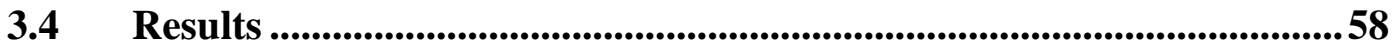

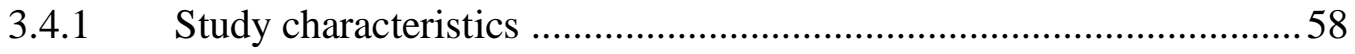

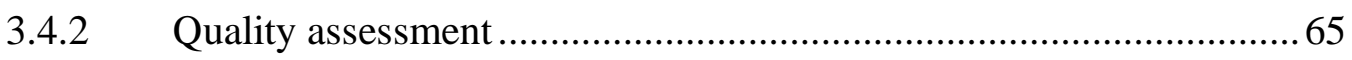

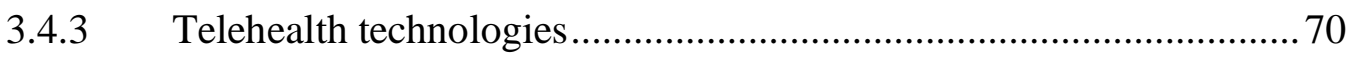

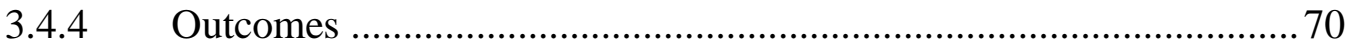

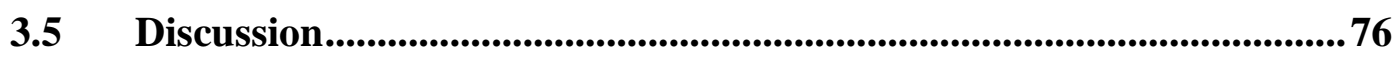

3.5.1 The strengths, limitations and future direction of this review ...........77

3.5.2 The implications of the findings for practice .................................. 78 
3.6 Conclusion........................................................................................

3.7 Findings to be taken forward .....................................................................79

$3.8 \quad$ Summary .....................................................................................................................8

Chapter 4: Technology Acceptance Models and Theories ........................................81

$4.1 \quad$ Introduction ......................................................................................................8

4.2 Technology acceptance ......................................................................................81

4.3 Technology acceptance models and theories ………………………..............83

4.3.1 Diffusion of Innovation Theory (DOI) ……………………………...83

4.3.2 Theory of Reasoned Action (TRA) ……………………………........ 84

4.3.3 Theory of Planned Behaviour (TPB) ……………………………….... 86

4.3.4 Technology Acceptance Model (TAM) ……………………………....8

4.3.5 Unified Theory of Acceptance and Use of Technology (UTAUT) ...90

4.4 Summary .................................................................................................................. 102

Chapter 5: Methodology of Study (2) and Study (3)..............................................103

$5.1 \quad$ Introduction .............................................................................................................. 103

$5.2 \quad$ Research design ................................................................................................. 103

5.3 Study (1): Use of Telehealth for Facilitating the Diagnostic Assessment of Autism Spectrum Disorder (ASD): A Scoping Review. ..................................107

5.4 Study (2): Exploring Parents/Carers Experience of Receiving a Diagnosis of Autism Spectrum Disorder (ASD) for their Children in Wales. 107

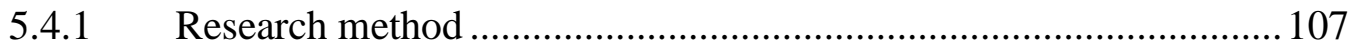

5.4.2 Population and sample ………………………………................... 108

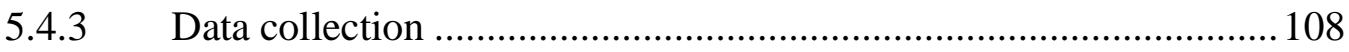

5.4.4 Encouraging participation ............................................................. 114

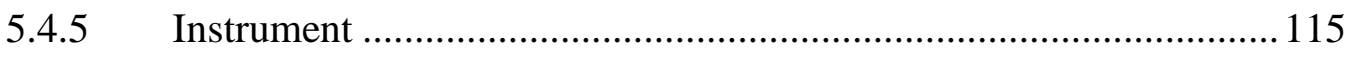

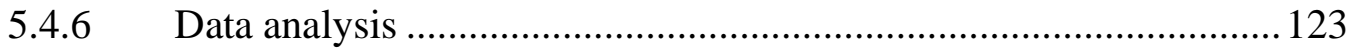

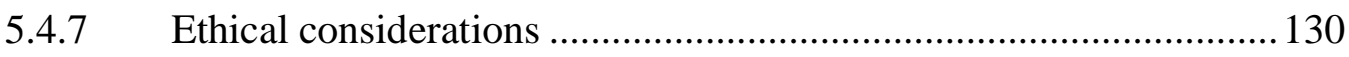

5.5 Study (3): Exploring Parents/Carers Acceptance of Using NODA for

ASD Diagnostic Assessment for their Children in Wales..................................132 
5.5.1 Developing a Hypothesised Model

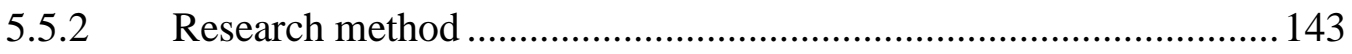

5.5.3 Population and sample .................................................................. 144

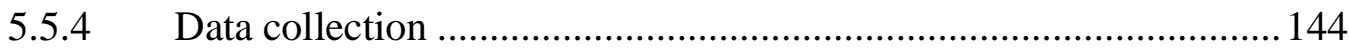

5.5.5 Encouraging Participation........................................................... 144

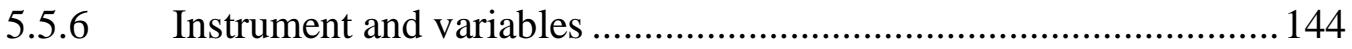

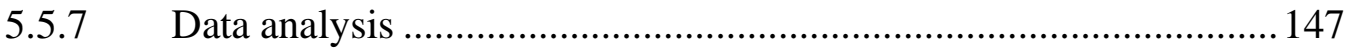

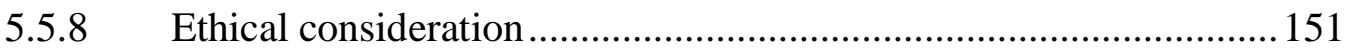

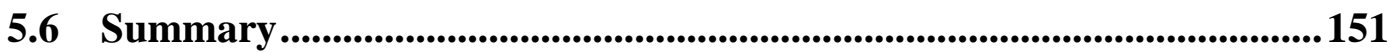

Chapter 6: Study (2) Results: Exploring the Parents/Carers Experience of Receiving a Diagnosis of Autism Spectrum Disorder for their Children in Wales.

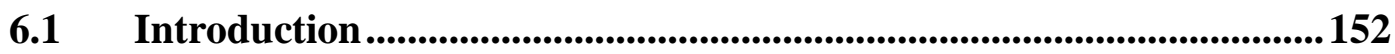

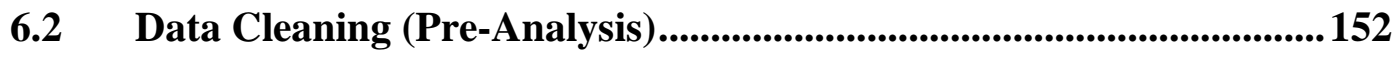

6.3 Demographics of the parents/carers ....................................................153

6.4 General information about the child with ASD ...............................155

6.5 The diagnostic process ........................................................................ 157

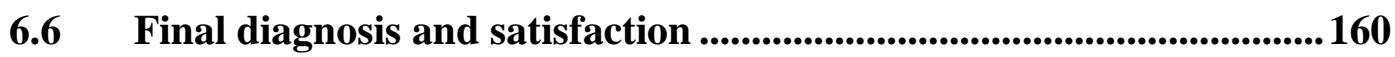

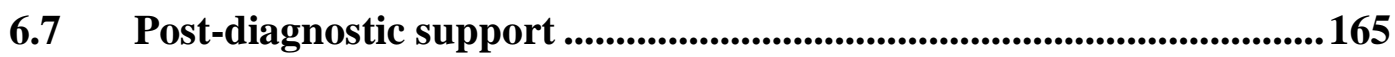

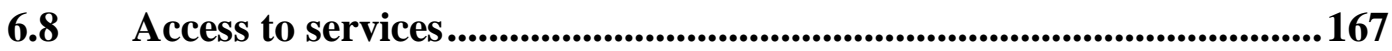

6.8.1 Reasons why parents/carers experienced difficulties/delays in accessing ASD related services:

6.9 Factors associated with parent/carer satisfaction with the overall diagnostic process on univariate analysis ..........................................................176

6.10 Predictors of parent/carer satisfaction with the diagnostic process.. 179

6.10.1 Assumptions of the binary logistic regression...... 179

6.10.2 Parent/carer satisfaction with the overall diagnostic process of ASD model (with missing data)

6.10.3 Parent/carer satisfaction with the overall diagnostic process of ASD model (after imputation) 
Chapter 7: Study (3) Results: Exploring Parents/Carers Acceptance of Using

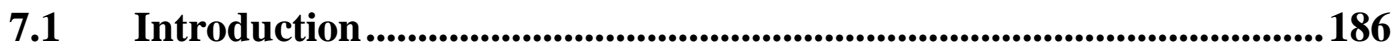

7.1 Demographics of the parents/carers .................................................. 187

7.2 General information about the child with ASD ...............................187

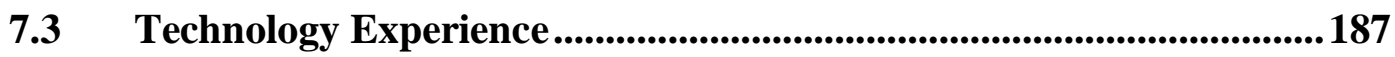

7.4 Parent/carer perceptions on NODA using UTAUT/added constructs 190

7.4.1 Potential reasons of parents/carers who felt they would have used the NODA system:

7.4.2 Potential reasons of parents/carers who felt they would not have used the NODA system: .....

7.5 Parents/carers anticipated usage of NODA ............................................208

7.6 Reliability of the constructs..............................................................209

7.7 Univariate analysis of the association of variables on behavioural

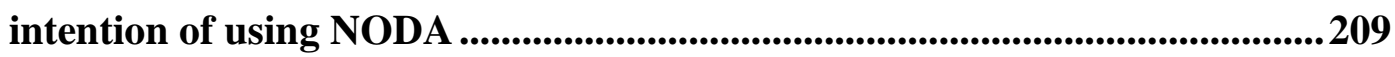

7.8 Determination of predictors of parent/carer intention to use the NODA for diagnostic assessment in Wales ..............................................212

7.8.1 Behavioural intention of parents/carers to use the NODA model (with missing data)

7.8.2 Parents/carers behavioural intention to use the NODA model (after

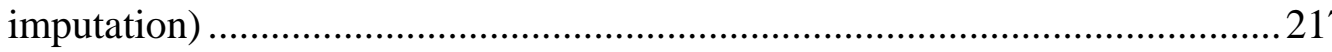

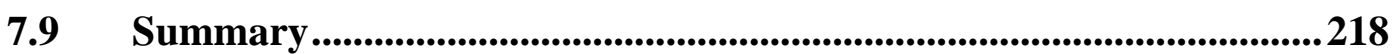

Chapter 8: Discussion ..........................................................................................221

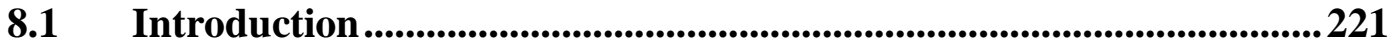

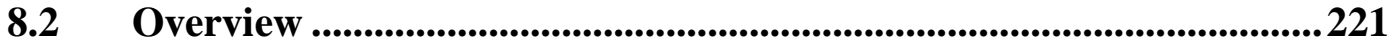

8.3 Study (2): Exploring the Parent/Carer Experience in Wales of Receiving a Diagnosis of Autism Spectrum Disorder for their Children .....223

8.3.1 Experience of parents/carers with the diagnostic process ...............223

8.3.2 Factors associated with the satisfaction levels of parents/carers with

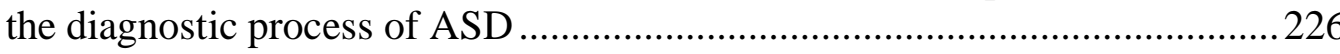


8.3.3 Predictors of parent/carer satisfaction with the diagnostic process of ASD 234

8.4 Study 3: Exploring the Acceptance Levels of Parents/Carers in Wales of Using NODA for ASD Diagnostic Assessment of their Children. ..............236

8.4.1 Existing direct determinants within UTAUT model ......................237

8.4.2 Added direct determinants in amended UTAUT model ..................241

8.4.3 Potential reasons for parent/carer perceptions toward the NODA system 247

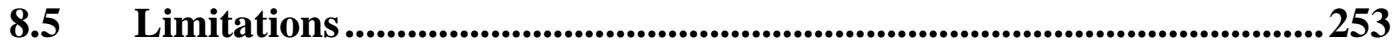

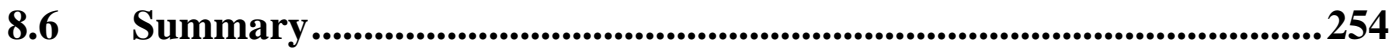

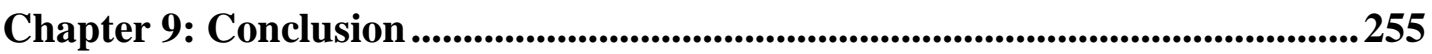

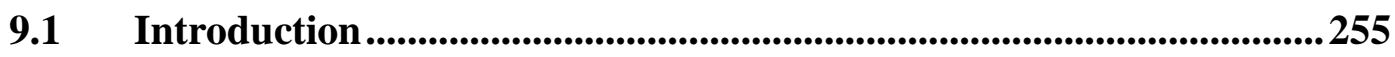

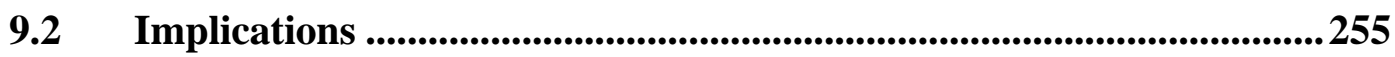

9.3 Recommendations for future research .................................................259

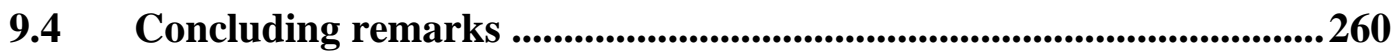

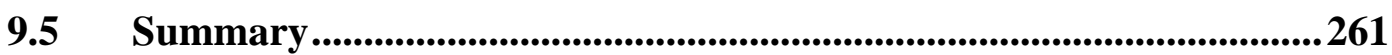

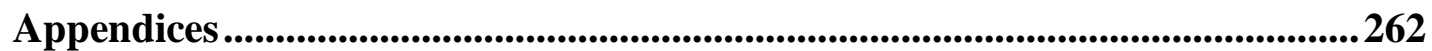

Appendix 1: Research Article: Use of Telehealth for Facilitating the Diagnostic Assessment of Autism Spectrum Disorder (ASD): A scoping

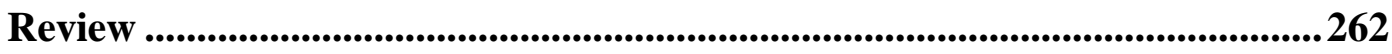

Appendix 2: (PRISMA-ScR) Checklist .....................................................2278

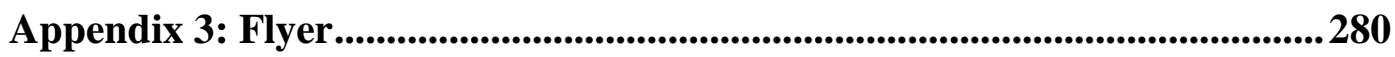

Appendix 4: Participant Information Sheet ...................................................281

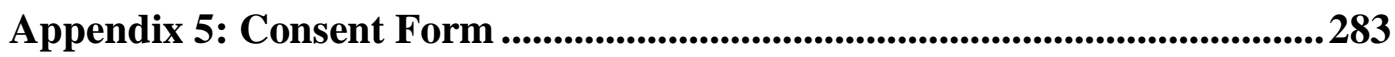

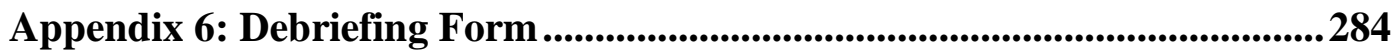

Appendix 7: Online Questionnaire ......................................................................2285

Appendix 8: Ethical Approval .............................................................................314

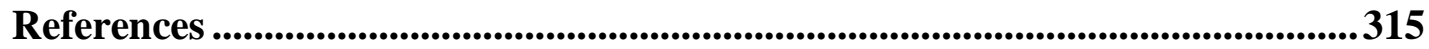




\section{List of Tables}

Table 3-1: Summary of study characteristics 60

Table 3-2: Results of quality assessment tool (from CASP) for diagnostic studies. ..66

Table 3-3: Results of quality assessment tool (from the NHLBI) for observational cohort and cross-sectional studies

Table 3-4: Results of quality assessment tool (from CASP) for qualitative study

Table 4-1: Frequency of theories and models used to predict end-user acceptance of telehealth use (Harst et al., 2019)

Table 4-2: The determinants of individual acceptance models and theories. Adapted from (Venkatesh et al., 2003)

Table 4-3: Explained Variance in the Models TAM, TAM2, TAM3, and UTAUT.....94

Table 4-4: Items used in estimating UTAUT (Venkatesh et al., 2003) 100

Table 5-1: The Autism-related Groups that were contacted in the Facebook.....

Table 5-2:Questionnaire Sections (Part 1) and Variables.

Table 5-3: The variables and items used in the questionnaire

Table 6-1: Demographics of the parent/carer 154

Table 6-2: General information about the child with ASD. 156

Table 6-3: The diagnostic process

Table 6-4: Final diagnosis and satisfaction 162

Table 6-5: Post-diagnostic support 166

Table 6-6: Access to services

Table 6-7: Univariate analysis of the association of variables on satisfaction with overall diagnostic process of ASD

Table 6-8: Binary Logistic Regression Model (with missing data) of Parents/carers satisfaction with the overall diagnostic process of ASD model

Table 6-9: Binary Logistic Regression Model (with imputed data) of parents/carers satisfaction with the overall diagnostic process of ASD model

Table 7-1: Parents'/carers' experience with technology 
Table 7-2: Perceptions of parents/carers on NODA using UTAUT/added constructs

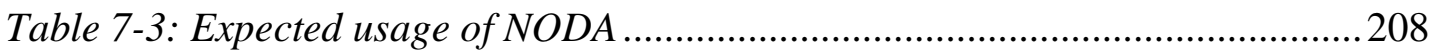

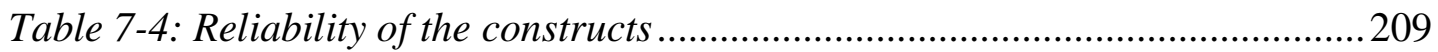

Table 7-5:Univariate analysis of the association of variables on Behavioural Intention of using NODA

Table 7-6: Binary Logistic Regression Model (with missing data) of the factors that associate with the parents'/carers' intention to use the NODA for diagnostic assessment in Wales

Table 7-7: Binary Logistic Regression Model (with imputed data) of the factors that associate with the parents'/carers' intention to use the NODA for diagnostic assessment in Wales 


\section{List of Figures}

Figure 1-1: Map of Wales, source: (WorldAtlas, 2020) ...........................................5

Figure 1-2: The percentage of people using digital technology to support their health in Wales (Davies et al., 2019).

Figure 1-3: The percentage of people using digital technology to monitor their health in Wales (Davies et al., 2019).

Figure 2-1: Estimated ASD population figures for the UK and the four nations based on 2011 census figures (National Autistic Society, 2017a)

Figure 2-2: Pathway for recognition, referral and diagnostic assessment of possible ASD of individuals under 19 years old (National Institute for Health and Care Excellence, 2017)

Figure 3-1: Flowchart of study selection process

Figure 4-1: Basic concept underlying user acceptance models and theories

(Venkatesh, Morris, Davis, \& Davis, 2003).

Figure 4-2: Innovation Decision Process (Rogers, 2003). 84

Figure 4-3: Theory of Reasoned Action (TRA) (Ajzen \& Fishbein, 1980; Fishbein \& Ajzen, 1975)

Figure 4-4: Theory of Planned Behaviour (TPB) (Ajzen, 1985) .86

Figure 4-5: Technology Acceptance Model (TAM) (Davis, 1989)

Figure 4-6: Technology Acceptance Model 2 (TAM2) (Venkatesh \& Davis, 2000)..89

Figure 4-7: Technology Acceptance Model 3(TAM3) (Venkatesh \& Bala, 2008) ....90

Figure 4-8: Unified Theory of Acceptance and Use of Technology (UTAUT)

(Venkatesh et al., 2003). .95

Figure 5-1: Research Design 106

Figure 5-2: Proposed Model of Parents/Carers Behavioural Intention to Use NODA

System based on the UTAUT Model 141

Figure 5-3: NODA SmartCapture: Home screen showing 4 NODA scenarios

(Nazneen et al., 2016)

Figure 5-4: NODA SmartCapture: Each scenario includs a 'sample video' and recording instructions about desired interactions with the child during recording (Nazneen et al., 2016) 
Figure 5-5: NODA SmartCapture: after going throughout instructions, parents can start video recording. Video can be stopped any time or it stops automatically after 10 minutes (Nazneen et al., 2016)

Figure 5-6: NODA SmartCapture: Home screen shows status of the recorded scenario with options to view, delete or send video to diagnostician. Once video is sent it uploads to NODA (Nazneen et al., 2016) ......................................... 146

Figure 5-7: NODA Connect: Diagnostic report (Nazneen et al., 2016).................. 146

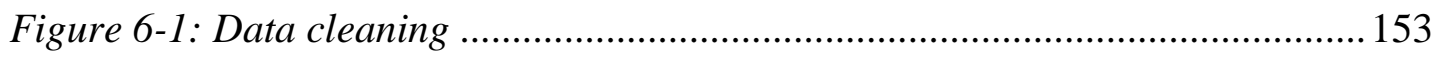

Figure 6-2: Professional who had been seen by parent/carer at the first time ....... 158

Figure 6-3: Parents/carers satisfaction with the time taken between first seeking help and receiving a diagnosis of ASD ................................................................. 164

Figure 6-4:Parents/carers satisfaction with the diagnostic process as whole ......... 164

Figure 6-5: Reasons why parents/carers experienced difficulties/delays in accessing

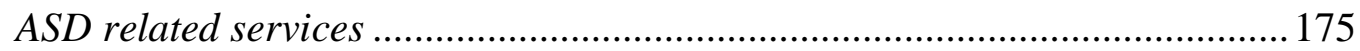

Figure 7-1:Parents/carers comfort with using a Desktop Computer ...................... 188

Figure 7-2: Parents/carers comfort with using a Laptop Computer ....................... 188

Figure 7-3: Parents/carers comfort with using a Tablet Computer ........................ 189

Figure 7-4: Parents/carers comfort with using a Mobile Phone/Smartphone ........ 189

Figure 7-5: Potential reasons of parents/carers who felt they would have used the

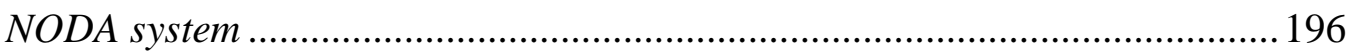

Figure 7-6: Potential reasons of parents/carers who felt they would not have used the NODA system ....................................................................................... 203

Figure 7-7: Parents/Carers Behavioural Intention to Use the NODA Model. .........216

Figure 8-1: Summary of the main results. 252 


\section{Abbreviations}

ABA: Applied Behaviour Analysis.

ADDM: Autism and Developmental Disabilities Monitoring.

ADHD: Attention Deficit Hyperactivity Disorder.

ADI-R: Autism Diagnostic Interview - Revised.

ADOS-2: Autism Diagnostic Observation Schedule, Second Edition.

ADOS: Autism Diagnostic Observation Schedule.

AIMS: Anaesthesia Information Management System.

ASD: Autism Spectrum Disorder.

ATA: American Telemedicine Association.

CI: Confidence Interval.

CT: Computerised Tomography.

DF: Degrees of Freedom

DOI: Diffusion of Innovation Theory.

DSM-5: Diagnostic and Statistical Manual of Mental Disorders, 5th Edition.

DSM-IV: Diagnostic and Statistical Manual of Mental Disorders: Text Revision.

ECG: Electrocardiograph.

ECHO: Echocardiography.

EHR: Electronic Health Record.

EMR: Electronic Medical Record.

ESDM: Early Start Denver Model.

FA: Functional Analysis.

FCT: Functional Communication Training.

FII: Fabricated or Induced Illness.

GP: General Practitioner.

ICD-10: International Classification of Diseases, 10th Revision.

ICD-11: International Classification of Diseases, 11th Revision.

ICIS: Intensive Care Information System.

ICTs: Information Communication Technologies.

ISUT: Integrated System Using Telemedicine.

ITV: Interactive Tele-videoconferencing.

IVC: Interactive Videoconferencing.

M: Mean. 
MAR: Missing at Random.

MI: Multiple Imputation.

MPCU: Model of Personal Computer Utilization (MPCU).

NHS: National Health Service.

NICE: National Institute for Health and Care Excellence.

NODA: Naturalistic Observation Diagnostic Assessment.

OR: Odds Ratio.

PDDNOS: Pervasive Developmental Disorder-Not Otherwise Specified.

PTSD: Post-Traumatic Stress Disorder.

SD: Standard Deviation.

SEN: Special Educational Needs.

TAM: Technology Acceptance Model.

TPB: Theory of Planned Behaviour.

TRA: Theory of Reasoned Action.

UTAUT: Unified Theory of Acceptance and Use of Technology.

VISYTER: Versatile and Integrated System for Tele-rehabilitation.

WHO: World Health Organisation. 


\section{Chapter 1: Introduction}

\subsection{Introduction}

This chapter provides an introduction to the current research study. It begins by presenting the context of the study, the study setting and population, the specific problem to be investigated and the overall significance of the study. This is followed by the aim and objectives and a clear outline of the thesis structure. Finally, a summary of this chapter is presented.

\subsection{Context of the study}

Autism Spectrum Disorder (ASD) is a lifelong neurodevelopmental condition, characterised by impairment in terms of social communication and social interaction and a repetitive and restricted pattern of interest, behaviour and activity (American Psychiatric Association, 2013b). Autism is a spectrum condition. This means that while all individuals with ASD may share many similar difficulties, the condition impacts upon each one in a different way, meaning that they need varying levels of support (Happé \& Ronald, 2008; Lai, Lombardo, Chakrabarti, \& Baron-Cohen, 2013). Although any psychiatric disorder typically includes heterogeneity, the amount of heterogeneity in ASD surpasses those found in other psychiatric disorders (Waterhouse, 2013).

The prevalence of ASD appears to be increasing globally (Centers for Disease Control and Prevention, 2018; Hansen, Schendel, \& Parner, 2015; Lai, Lombardo, \& BaronCohen, 2014). Around 1 in 54 children in the United States has been diagnosed with ASD, according to estimates from the Centers for Disease Control and Prevention's Autism and Developmental Disabilities Monitoring (ADDM) Network (Maenner, Shaw, \& Baio, 2020). With an estimated number of 700,000 people in the United Kingdom, diagnosing the condition of autism represents a pressing public health and clinical care challenge (Crane, Chester, Goddard, Henry, \& Hill, 2015; National Autistic Society, 2018a). 
It has been shown that ASD begins in childhood and typically persists into adolescence and adulthood (World Health Organisation, 2019). While ASD is a condition that commonly impacts an individual from childhood through to adulthood, etiological aspects and therapeutic strategies have not yet been fully identified or understood (Baudouin, 2014; Gottfried, Bambini-Junior, Francis, Riesgo, \& Savino, 2015). Most importantly, ASD has a significant effect on several areas of a child's development and functioning. All this means that the impact of ASD on a child and family is considerable.

Research has shown that there is a substantial delay, approximately 20 months to 5 years, between parents' first noticing concerns about the development of their child and receiving a confirmed diagnosis of ASD (Crane et al., 2015; Howlin \& Asgharian, 1999; Howlin \& Moore, 1997; Nazneen, Matthews et al., 2015; Wiggins, Baio, \& Rice, 2006). This may result in delayed access to early intervention services, which are critical for ensuring positive outcomes (Matson, 2008; McEachin, Smith, \& Ivar Lovaas, 1993; Rattazzi, 2014; Siegel, 2008; Smith et al., 2017). Long wait times are also the cause of significant stress to individuals and families (Crane et al., 2015a; Hayes \& Watson, 2013; Siklos \& Kerns, 2007). Some of the reasons for delays in diagnosis include the overall shortage of healthcare professionals (Thomas, Ellis, McLaurin, Daniels, \& Morrissey, 2007) and several specialist and treatment appointments are often needed to make a diagnosis. Multiple meetings with a variety of professionals in different locations can also be very stressful for individuals who might later be diagnosed with ASD, as people with ASD are very sensitive to any change in their situations and extremely reliant on routines (American Psychiatric Association, 2013a). Some families have to travel long distances in order to access these services (Oberleitner, Elison-Bowers, Reischl, \& Ball, 2007). This means that there is not only a cost to the family but also to the specialist healthcare teams who must travel in order to reach different areas. Overall, there seems to be some significant evidence to indicate that parents of children with ASD face considerable difficulty in obtaining a timely diagnosis of ASD for their child. Therefore, there is a pressing need for any potential solutions that could support parents and their children who are in need of such services. 
Since the 1960s, Information Communication Technologies (ICTs) have been identified as a means of improving not only the quality of healthcare services offered, but also facilitating access to such services (Fichman, Kohli, \& Krishnan, 2011; Ortiz \& Clancy, 2003). In particular, telehealth can provide help and support to people with specific health and well-being needs. Telehealth is defined as a mechanism that allows people to receive professional help and support from a distance (Vismara, McCormick, Young, Nadhan, \& Monlux, 2013). Telehealth approaches have recently been explored as a way of supporting the delivery of a range of services for people with ASD, including remote parent training and behavioural intervention and treatment (Ingersoll \& Berger, 2015; Pickard, Wainer, Bailey, \& Ingersoll, 2016; Vismara et al., 2013; Wacker, Lee, Dalmau, Kopelman, Lindgren, Kuhle, Pelzel, Dyson, et al., 2013; Wainer \& Ingersoll, 2015). Telehealth can offer a possible solution to improve the diagnostic pathway of ASD and reduce the time taken to receive a final diagnosis (Nazneen, Matthews et al., 2015; Reese, Jamison et al., 2015).

The successful implementation of clinical information technology systems, including telehealth, is highly reliant on user acceptance (Davis, 1993; Kowitlawakul, 2011). Research has indicated that the way in which the potential user perceives telehealth is a key factor in its successful adoption (Hu, Chau, Sheng, \& Tam, 1999; Kim, Gellis, Bradway, \& Kenaley, 2018). New technology may not be successful if potential users are dissatisfied with it. Therefore, it is critical to understand whether parents are willing to use telehealth as a means of diagnostic assessment of ASD. In spite of the obvious importance of parents' perceptions towards telehealth as a mechanism for remote ASD diagnosis, to the researcher's knowledge, no study has been conducted that examines telehealth adoption for ASD diagnostic assessment in Wales. On account of this knowledge gap, the current thesis examines the predictors of parental acceptance around the use of telehealth for ASD diagnosis. In order to determine the acceptance of telehealth amongst parents/carers in Wales, it was crucial to initially explore their experiences whilst obtaining a confirmed diagnosis for their child. These findings will highlight the existing challenges that face parents/carers throughout the diagnostic process and help in understanding their openness towards the use of telehealth as a means of diagnostic assessment. Furthermore, it is hoped that this could help in presenting some useful recommendations for improving the diagnostic pathway of ASD in Wales. 


\subsection{Study setting and population}

This research was conducted in Wales. Wales is located on the island of Great Britain, and lies to the west of England. It covers an area of 20,779 square kilometres (8,030 square miles) (see Figure 1-1) (WorldAtlas, 2020). The horizontal width is $149 \mathrm{~km}$ (92 miles) from St. David's east towards Hay on Wye. The vertical length is $201 \mathrm{~km}$ (124 miles) from Rhyl, directly south to the capital city of Cardiff (WorldAtlas, 2020). In 2018, Wales was home to 3,138,000 residents. Roughly half of the Welsh population centered in South East Wales, covering just 14 percent of the land area (Welsh Government, 2020). The most populated authority in Wales is Cardiff, with approximately 364,000 residents in 2018, representing almost $24 \%$ of the population of the South-East Wales region. This region also comprises the authority with the smallest population in Wales, which is Merthyr Tydfil, with nearly 60,000 residents (Welsh Government, 2020). In 2018, Wales had an average population density of 151.4 persons per square kilometre (Welsh Government, 2020).

Wales is subdivided into 22 unitary authorities (Office for National Statistics, 2016; WorldAtlas, 2020). They are Blaenau Gwent, Bridgend, Caerphilly, Cardiff, Carmarthenshire, Ceredigion, Conwy, Denbighshire, Flintshire, Gwynedd, Isle of Anglesey, Merthyr Tydfil, Monmouthshire, Neath Port Talbot, Newport, Pembrokeshire, Powys, Rhondda Cynon Taf, Swansea, Torfaen, Vale of Glamorgan, and Wrexham. Eight of the unitary authorities (Bridgend, Cardiff, Merthyr Tydfil, Neath Port Talbot, Newport, Torfaen, Wrexham and Rhondda Cynon Taf) have county borough status (reflecting the fact that they are large centres of population). The other 14 have county status (reflecting some characteristic of rurality).

However, these definitions do not impact upon authority structures (Office for National Statistics, 2016). The following section outlines the problem. 


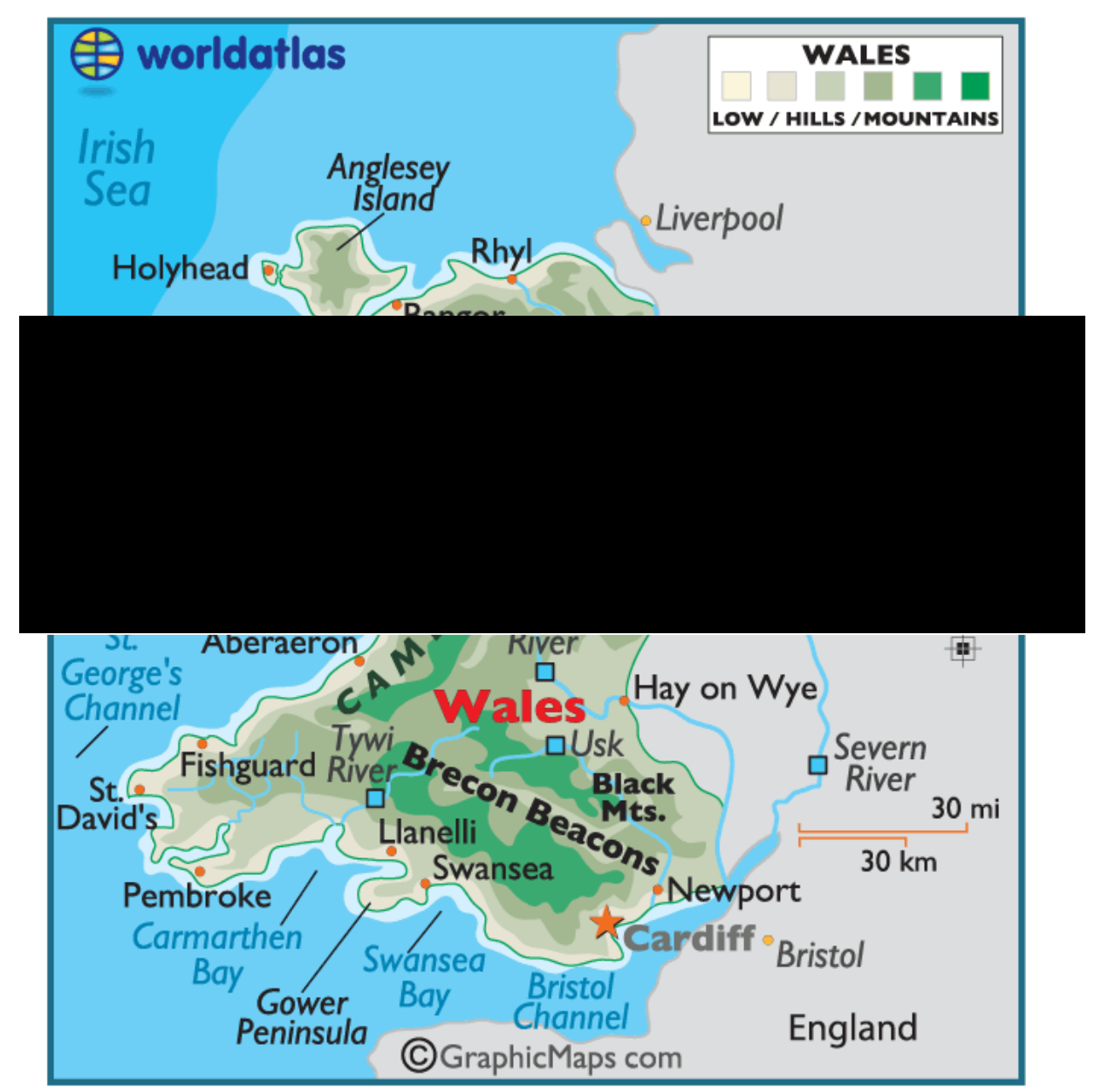

Figure 1-1: Map of Wales, source: (WorldAtlas, 2020)

\subsection{Statement of the problem}

Research has shown that parents of children with ASD face long delays between seeking professional help and obtaining a diagnosis of ASD (Crane et al., 2015; Howlin \& Asgharian, 1999; Howlin \& Moore, 1997; Nazneen, Matthews et al., 2015; Wiggins et al., 2006). The situation in Wales is no exception, with parents facing welldocumented difficulties around ASD assessment and diagnosis. For example, during the consultation on the Refreshed Autistic Spectrum Disorder Strategic Action Plan, parents voiced their concerns over the challenges they faced when seeking a referral to diagnostic assessment services through either primary healthcare services or schools (Welsh Government, 2016). They were concerned that they could not obtain a speedy referral for diagnostic assessment for their child, and without a confirmed diagnosis they were unable to receive the necessary help and support services (Welsh Government, 2016). 
Furthermore, Wales comprises of several large rural areas, which is home to approximately one third of the population (The Welsh NHS Confederation, 2018). Access and delivery of healthcare services are particularly challenging in such rural areas (Norton, Longley, \& Ponton, 2012). People living in these communities must often travel much further to access healthcare services, than those who live in urban areas (The Welsh NHS Confederation, 2018). For those people without their own method of transport, this is even bigger issue. Around 11\% of households in rural Wales do not have the use of a motor vehicle (Norton et al., 2012). It has been shown that more than twice the number of people without a car have difficulty accessing healthcare services when compared with those with private transport (Norton et al., 2012). Moreover, there is significantly higher costs to providing healthcare services for large, sparsely populated rural areas (The Welsh NHS Confederation, 2018). In an attempt to decrease travel times for patients in rural areas, some healthcare services are offered in several locations, whereas in more populated areas the same services could be provided in just one location. This could mean that a greater number of healthcare professionals are needed to deliver healthcare services in rural communities (The Welsh NHS Confederation, 2018).

There is clearly a pressing need to support those parents and children in need of ASD diagnostic services in Wales, particularly to enable them to obtain earlier diagnoses which would in turn make access to early intervention services easier. Previous research suggests that telehealth could be a possible means of addressing the need for improved access to services for individuals with ASD (Boisvert, Lang, Andrianopoulos, \& Boscardin, 2010). In particular, telehealth may help to improve the diagnostic process of ASD and support diagnostic assessment (Nazneen, Matthews, et al., 2015; Reese, Jamison, et al., 2015).

However, there is limited existing research regarding the degree to which parents will accept telehealth as a means of remote ASD diagnosis. Lack of acceptance by the user is the main barrier to the success of any new information systems (Davis, 1993; Dillon \& Morris, 1996). User acceptance is considered as the essential factor that can determine either the success or failure of any information system schemes, including telehealth (Davis, 1993; Hu et al., 1999). There is obviously a clear need to investigate the acceptance of parents/carers towards telehealth technology, which could facilitate 
access to diagnostic services and receive an earlier diagnosis for their child. A clear understanding of such adoption is essential in order to encourage the widespread acceptance and use of digital technologies in Wales, that could improve access to diagnostic services. Otherwise, if no such understanding is obtained, any telehealth technology implemented may not succeed, its efficacy could decline and not be sustainable long term (Davis, 1993; Dillon \& Morris, 1996; Hu et al., 1999). Furthermore, in time this may lead to the loss of important resources such as time and money.

In order to examine the predictors of parents/carers acceptance of using telehealth for diagnostic assessment, it is important to firstly explore their experience with the diagnostic process in Wales, specifically following the Refreshed Autistic Spectrum Disorder Strategic Action Plan (Welsh Government, 2016). The assessment and diagnosis of ASD was one of the plan's main priority areas for action. The intention was to deliver improvements in the assessment and diagnostic services for children, by creating a national standardised assessment pathway, in order to ensure consistent provision for all children. In addition, so as to improve timeliness of access, they set a target of 26 weeks waiting time from initial referral to the first assessment appointment (Welsh Government, 2016).

Therefore, in light of such supposed improvements to the ASD diagnostic services in Wales, it was timely to conduct an exploration into parental experiences of obtaining an ASD diagnosis in Wales. The aim of this was to investigate their various experiences with the diagnostic process, their levels of satisfaction, and whether children were being diagnosed more rapidly and at an earlier age.

\subsection{Significance of the study}

Although the use of telehealth in the assessment and diagnosis of some conditions is well established (Bowman, Kennedy, Kirwan, Sze, \& Murdoch, 2003; McCrossan et al., 2008; Shores et al., 2004), the use of telehealth to support access to the assessment and diagnosis of ASD is in its infancy. This means that there is a need to understand the degree to which potential users will accept such technology, before it is fully implemented. Identification of the factors that may influence this acceptance is 
necessary in order to ensure success (Davis, 1993; Hu et al., 1999). The determinants of the levels of acceptance towards using telehealth as a method of ASD diagnosis, have not yet been well studied, with only a small body of research currently in existence. A review of these studies indicated that, to date, no study has examined the factors based on the theoretical foundation that drives the levels of acceptance of such telehealth (More information on this will be presented in Chapter 3).

This study intends to fill that knowledge gap and builds on previous work by investigating the driving factors of the acceptance of the use telehealth for remote diagnosis. This study will use the Unified Theory of Acceptance and Use of Technology (UTAUT) model (Venkatesh et al., 2003) as a theoretic framework to examine the determinants that may influence the intention to use telehealth. The outcomes of this study will contribute to a comprehensive model that can facilitate a better understanding of the parents/carers perceptions toward the use of telehealth for remote diagnostic assessment. This research will make a significant contribution to the literature, as no prior study has measured the parents intention to use telehealth for remote diagnosis using theoretical model. The predictors of acceptance must be investigated in order to increase the use of the telehealth diagnostic approach. This can then to promote best practice, enhancing the quality of life of autistic children and their families by enabling them to obtain easier and quicker access to ASD diagnostic services in Wales. The outcomes of this research would be of great value to parents and their children, diagnosticians and other professionals.

Such an approach would fit in with Wales' vision of using digital technology to support the health and well-being of the population. The National Strategy for Welsh Government, Prosperity for All (Welsh Government, 2017) highlights the importance of harnessing technology in order to improve access to health and social care services. The Welsh Government's Plan for Health and Social Care, A Healthier Wales (Welsh Government, 2018), also emphasises that delivering such a vision will include using technology to support high quality, sustainable services, including accessing health information and health services, monitoring and diagnosing symptoms. The strategy aims to ensure "a future where staff, service users, patients and carers are empowered and better informed, where organisational boundaries are not a barrier to effective care, as information is available electronically and joined-up, and where innovation and 
improvement can flourish built on a solid technical infrastructure and a safe environment for those who work in or are served by health and care services in Wales" (Welsh Government, 2015, p. 5).

Recent research suggests that the Welsh population may be receptive to the use of telehealth. For example, the first national representative survey was carried out in Wales in 2018 with the aim of exploring social patterns of interaction with digital technologies for health purposes (Davies, Sharp, Homolova, \& Bellis, 2019). It revealed that two thirds $(66 \%)$ of the population in Wales aged 16 years and over, have used digital technology to support their health. The most common actions involved obtaining information about general health (57\%), about health services (53\%) about activities to support health (43\%), and to self-diagnose a health condition (34\%) (see Figure 1-2). Furthermore, one third (34\%) of the population in Wales who are aged 16 years and over, have used digital technology to monitor their health. The most commonly reported activities involved monitoring physical health such as step counting (28\%), fitness (27\%) and nutrition/food intake (21\%) (see Figure 1-3). The findings indicate that certain groups within the general population of Wales are already engaged in the use of digital technology to manage and monitor their health. Therefore, exploring parents/carers acceptance of the use of telehealth for remote ASD diagnosis of their children would align with Wales' overall strategy of using digital technology for improving access to health information and services.

In order to measure the degree to which parents/carers might accept the use of telehealth for diagnosis of ASD, was first necessary to understand the current situation and identify the parents/carers experience including any issues and barriers they are currently facing in relation to diagnostic services. There is a clear knowledge gap around this, particularly in Wales. This part of study will therefore aim to address this issue, to hopefully reduce this knowledge gap and obtain much-needed information. The following section outlines the study's aim and objectives. 


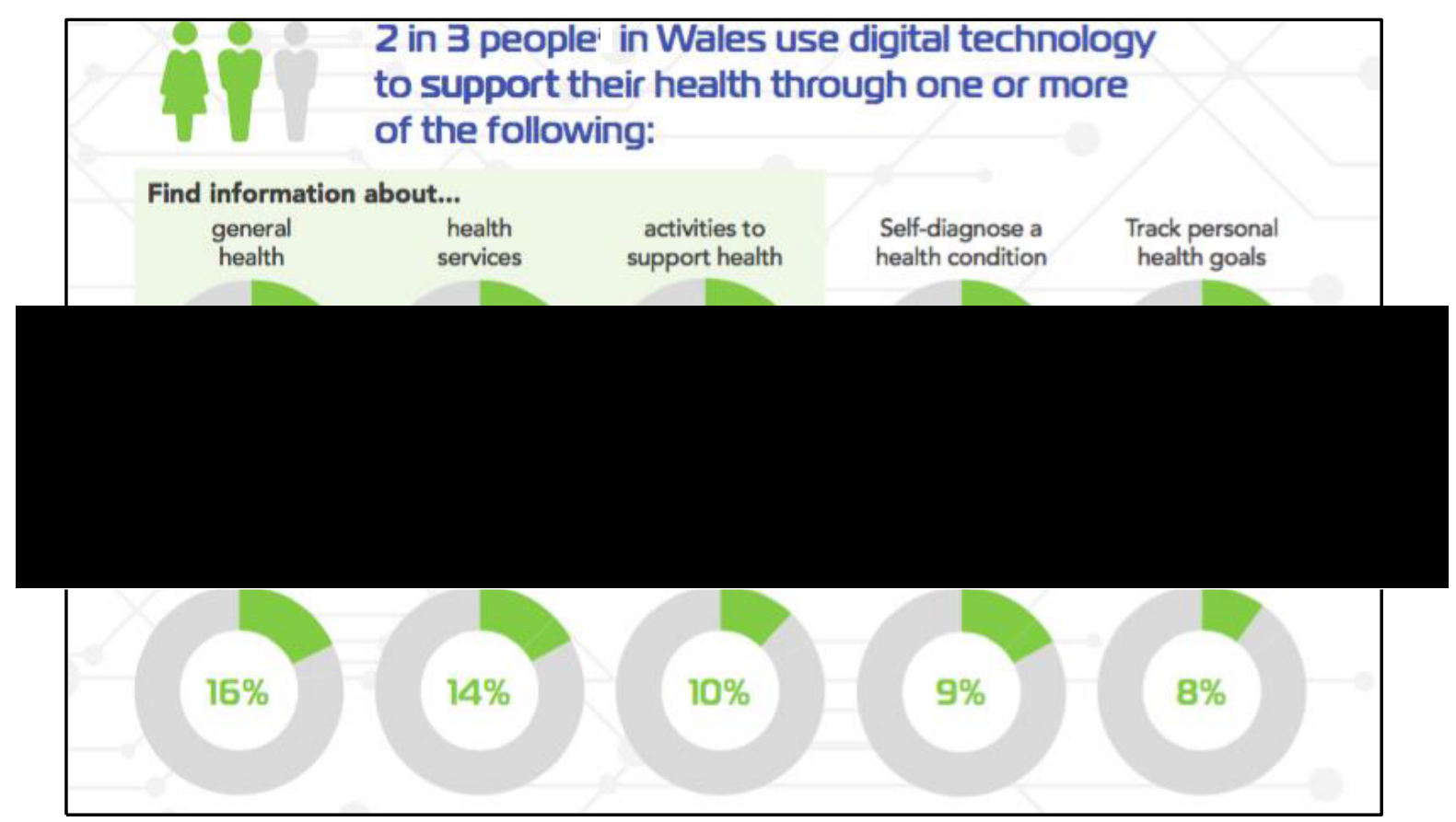

Figure 1-2: The percentage of people using digital technology to support their health in Wales (Davies et al., 2019)

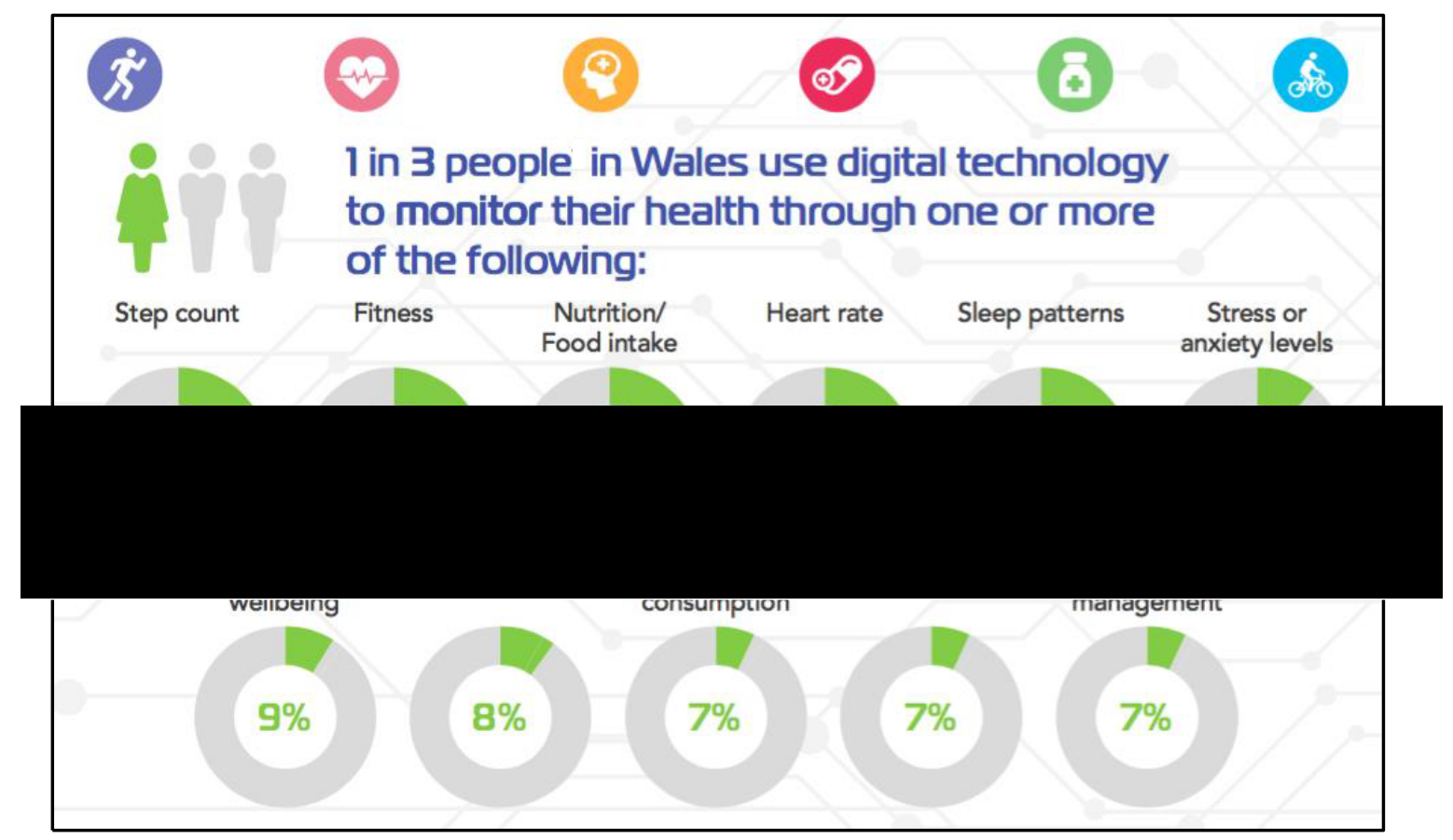

Figure 1-3: The percentage of people using digital technology to monitor their health in Wales (Davies et al., 2019) 


\subsection{Aim and objectives}

The literature indicates that there is a significant time lag between the age at which parents first become concerned about their child's development and the age at which the child obtains a final diagnosis (Crane et al., 2015; Howlin \& Asgharian, 1999; Howlin \& Moore, 1997; Nazneen, Matthews et al., 2015; Wiggins, Baio, \& Rice, 2006). It has been suggested that the use of telehealth could be a viable means of improving access to diagnostic ASD services for children, particularly those living in rural and underserved areas (Nazneen, Matthews et al., 2015; Reese, Jamison et al., 2015). However, studies have found that the overall attitude toward telehealth is a key indicator to telehealth adoption $(\mathrm{Hu}$, Chau, Sheng, \& Tam, 1999; Kim, Gellis, Bradway, \& Kenaley, 2018). Therefore, this research used a multimethod approach to examine whether telehealth technology can be used to increase access to ASD diagnostic services in Wales, thereby reducing the time taken to receive a confirmed diagnosis. It was important to conduct a scoping review at the outset, in order to identify the telehealth approaches currently used in the diagnosis and assessment of ASD, the feasibility and acceptability of those approaches, as well as the accuracy of remote diagnosis when compared with in-person methods (Objective 1). Then, in order to determine whether parents/carers of children with ASD are likely to adopt telehealth, it was vital to examine some of the broader issues and barriers they currently face in relation to the ASD diagnostic process and investigate the variables related to parental satisfaction that might impact upon their willingness to use telehealth. Little is known about these variables and most of the studies reviewed have only examined these factors independently (Objectives 2, 3, 4). Following that, the study sought to explore whether parents/carers would accept the use of telehealth as a means of remote diagnosis for their child by using the Unified Theory of Acceptance and Use of Technology (UTAUT) model (Objective 5). Specifically, the main aim of the research is:

Aim: to examine if telehealth technology can be used (e.g. the degree of acceptance by parents/carers) to increase access to ASD diagnostic services in Wales, UK and reduce the time taken to receive a diagnosis. 
To achieve this aim, the following objectives were defined:

1. To examine how telehealth is used in the diagnosis and assessment of ASD for children and adults, the feasibility and acceptability and the accuracy of the remote diagnosis when compared with face to face diagnosis and assessment methods.

2. To explore the experience of parents/carers in Wales when obtaining a confirmed diagnosis of ASD for their child.

3. To examine the significant factors associated with the parent/carer levels of satisfaction with the overall ASD diagnostic process in Wales.

4. To examine the predictors of parent/carer satisfaction with the overall ASD diagnostic process in Wales.

5. To examine the predictors of parent/carer intention to use telehealth for ASD diagnostic assessment in Wales. 


\subsection{Structure of the Thesis}

The thesis is structured as follows:

\section{Chapter 1: Introduction.}

The introductory chapter presents a general outline of the thesis. It includes the context of the study, a study setting and population, a statement of the problem, the significance of the study, the research's aim and objectives, and provides a summary of the thesis chapters.

\section{Chapter 2: Contributing Research Fields into ASD Diagnosis and Telehealth.}

This chapter presents a background of Autism Spectrum Disorder (ASD), its definitions and diagnostic criteria, co-occurring conditions, prevalence, the diagnostic process of ASD in the UK, and particularly in Wales and the ASD diagnostic instruments. Furthermore, it highlights the difficulties experienced by individuals and their families in obtaining a diagnosis of ASD, including reasons for the long delay between the seeking help and receiving a diagnosis. The factors associated with parental levels of satisfaction with the overall diagnostic process are also reviewed. This chapter also provides a background of telehealth definitions, communication technologies and various types of telehealth. It then also considers the use of telehealth within clinical specialities other than ASD.

\section{Chapter 3: Study (1) Use of Telehealth for Facilitating the Diagnostic Assessment of Autism Spectrum Disorder (ASD): A Scoping Review.}

This chapter presents a scoping review of the use of telehealth in ASD diagnostic assessment. The review's context, objectives, methods, results, discussion and conclusion are provided. This scoping review chapter appears before the methodology chapter as it was initially important to identify the telehealth approaches used for the diagnosis and assessment of ASD. One telehealth approach (NODA system) was specifically identified for exploring the attitudes of parents/carers toward receiving a remote diagnosis via telehealth. Chapter (4) then goes on to review the most widely used technology acceptance models and theories in order to identify one theoretical base model with which to study parents/carers willingness to use the NODA system for ASD diagnostic assessment. The methodology chapter refers to both the NODA system and the UTAUT model, making it necessary to present the scoping review and 
the technology acceptance chapters before the methodology, so as clarify the correct information and ensure the sequence amongst the chapters.

\section{Chapter 4: Technology Acceptance Models.}

The most influential technology acceptance theories and models are discussed and any limitations and shortcomings highlighted. The chapter ends with justification for the selection of the Unified Theory of Acceptance and Use of Technology (UTAUT) as a base model to study parents/carers acceptance of using the NODA system for ASD diagnostic assessment.

\section{Chapter 5: Methodology of Study (2) and Study (3).}

Chapter five presents the methodology of this research. It begins by describing the research design. This thesis includes three different but interrelated studies with different objectives. Because of this, the method for each one is described separately. Firstly, the scoping review (Study 1) is conducted in order to examine the use of telehealth approaches in the diagnostic assessment of Autism Spectrum Disorder (ASD). The method of scoping review is only alluded to briefly here, as it is fully described in Chapter 3. Then, Study (2) and Study (3) are conducted. The second study is related to exploring the parent/carer experience of the diagnostic process of ASD in Wales. The third study is related to exploring the degree to which parents/carers accept the use of telehealth for an ASD diagnostic assessment. The target population of the questionnaire were the parents/carers of children with ASD who live in Wales. It is important to explore the perspectives of all stakeholders including adults and health professionals towards using remote diagnostic of ASD. However, because of the limitations of both time and resources, it was decided to limit the research to parents/carers. In the future, research could be conducted amongst other populations in order to obtain a clearer overall picture regarding the acceptance of using telehealth for remote ASD diagnosis. Both studies begin by explaining the methods used and the data collection process. In Study (3) section, a hypothesised model development is presented. Both studies then consider the variables and instrument used and finally the ethical considerations and data analysis. 
Chapter 6: Study (2) Results. Exploring Parents/Carers Experience of Receiving a Diagnosis of Autism Spectrum Disorder for their Child in Wales.

This chapter presents the Study (2) findings of a survey which examines the experience of parents/carers in Wales of the diagnostic process of ASD, as outlined in Chapter 5. Descriptive and inferential findings are presented. Findings of open-ended questions are also provided.

Chapter 7: Study (3) Results. Exploring Parents/Carers Acceptance of Using Naturalistic Observation Diagnostic Assessment (NODA) System for ASD Diagnostic Assessment of their Child in Wales.

This chapter presents the Study (3) findings of a survey exploring the perceptions of parents/carers in Wales towards the use of NODA system for diagnostic assessment of ASD, as outlined in Chapter 5. Descriptive and inferential findings are presented. Findings of open-ended questions are also provided.

\section{Chapter 8: Discussion.}

This chapter presents a comprehensive discussion around the findings of the studies, both in terms of the research questions and also in relation to previous studies.

\section{Chapter 9: Conclusion.}

In this chapter, limitations of the thesis are highlighted, with an explanation of some theoretical and practical implications. Finally, it offers some recommendations for future research and concluding remarks.

A bibliography and appendixes related to this research is included at the end of the thesis. 


\subsection{Summary}

This introductory chapter has initially outlined the context of this research followed by the study setting and population, the statement of the research problem and the significance of the study. The study's aim and objectives were then discussed, followed by the structure of the thesis. The next chapter will provide a background on ASD. It will also emphasise the difficulties experienced by individuals and their families in obtaining a diagnosis of ASD. The factors contributing to parents' satisfaction with the overall diagnostic process will also be reviewed. This will then be followed with a background on telehealth and a review of its usage within different clinical specialties in addition to ASD. 


\section{Chapter 2: Contributing Research Fields into ASD Diagnosis and Telehealth}

\subsection{Introduction}

This chapter begins by providing an overview of Autism Spectrum Disorder (ASD), its definitions and diagnostic criteria, co-occurring conditions, prevalence, the diagnostic process of ASD in the UK, particularly Wales and the autism diagnostic instruments that are used to structure the information collected within a diagnostic assessment. It then goes on to highlight the difficulties in obtaining a diagnosis of ASD experienced by individuals and their families, including exploring the reasons for a long delay between seeking help and receiving a diagnosis. The factors contributing to the level of parent/carer satisfaction with the overall diagnostic process are then reviewed. Then follows a background of telehealth definitions, communication technologies and the various types of telehealth. It examines the use of telehealth in different clinical specialties, other than ASD. Finally, there is a summary for the chapter.

\subsection{Definitions and diagnostic criteria of ASD}

Autism is identified as being a developmental disability that impacts on how individuals interact and communicate with the world (National Autistic Society, 2018a). Autism was initially defined by Leo Kanner in 1943 as an inherent inability to interact normally with other individuals in a biologically predetermined way (Kanner, 1943). There have since been great advances in the concept of autism. In the third edition of the Diagnostic and Statistical Manual of Mental Disorders (DSM-III), autism was characterised by the absence of responsiveness to other individuals, language deficits, difficulties in communicating and inexplicable reactions to the different environmental stimuli, all of which begins during the first 30 months of life (United Psychiatric Association, 1980). 
The following modifications occurred in the fourth edition (DSM-IV), in which 'Pervasive Developmental Disorders' was an umbrella term including five diagnoses: Autistic Disorder, Asperger disorder, Pervasive Developmental Disorder Not Otherwise Specified (PDD-NOS), Rett's Disorder and Childhood Disintegrative Disorder (American Psychiatric Association, 2000). This list is similar to the International Classification of Diseases (ICD-10), in which a 'Pervasive Developmental Disorder' was a main heading including probable autism profiles, such as Atypical Autism, Childhood Autism and Asperger syndrome (World Health Organisation, 1993). The diagnostic criteria in DSM-IV and ICD-10 were identified by three features: impairments in patterns of communication, impairments in social interaction, and restricted, stereotyped, repetitive patterns of interests, behaviour and activities (American Psychiatric Association, 2000; World Health Organisation, 1993).

The latest revision of the Diagnostic and Statistical Manual of Mental Disorders "DSM-5", published in May 2013, approved the umbrella term Autism Spectrum Disorder (ASD) without any subtypes (American Psychiatric Association, 2013a). Accordingly, persons with approved DSM-IV diagnosis of Asperger disorder, Autistic Disorder, or Pervasive Developmental Disorder Not Otherwise Specified should be given the diagnosis of ASD. The reason for this change is that although these disorders have similar vital symptoms they occur with different degrees of severity (American Psychiatric Association, 2013a). Autism Spectrum Disorder (ASD) is defined by the American Psychiatric Association (2013b) as "a complex developmental condition that involves persistent challenges in social interaction, speech and nonverbal communication, and restricted/repetitive behaviours" (American Psychiatric Association, 2013b, para.4). The effects of ASD as well as the severity of symptoms are not the same in each individual (American Psychiatric Association, 2013b). The diagnostic criteria in DSM-5 are organised into two domains instead of three: deficits in social communication and social interaction and restricted and repetitive behaviour, interests, or activities (American Psychiatric Association, 2013a). 
The symptoms that describe the persistent deficits in social interaction and communication are as follow: (1) problems in reciprocating emotional or social interaction; (2) problems in nonverbal communication such as abnormal eye contact, facial expressions, posture and tone of voice; (3) problems in maintaining, understanding and developing relationships (American Psychiatric Association, 2013a). Two of the four subsequent symptoms related to restricted and repetitive behaviour must also be present for a diagnosis of ASD to be made: (1) stereotyped or repetitive speech, motor movements, or use of objects; (2) excessive adherence to routines, excessive resistance to change, or ritualised patters of verbal or nonverbal behaviour; (3) extremely restricted interests that are abnormal in intensity or concentration; (4) hyper-reactivity or hypo-reactivity to sensory input or infrequent interest in sensory features of the environment (American Psychiatric Association, 2013a). Symptoms must have been existent since early childhood in all cases, although they might not become fully clear until later on in life (American Psychiatric Association, 2013a; Ziats \& Rennert, 2016). The new International Classification of Diseases (ICD-11) was released by the World Health Organisation (WHO) in 2018, and it will come into effect in 2022 (World Health Organisation, 2018). The ICD-11 is now more aligned with DSM-5, as it involves Atypical Autism, Childhood Autism and Asperger syndrome as well as some other general developmental disorders, in the 'Autism Spectrum Disorder' category. It also involves the same two categories of autism diagnostic criteria as the DSM-5: difficulties in social communication and interaction, and restricted and repetitive behaviours and interests (World Health Organisation, 2018).

\subsection{Co-occurring conditions}

Many individuals diagnosed with ASD have co-occurring conditions (Lai et al., 2014). The most common of these conditions generally fall into four groups: developmental conditions, such as intellectual disability, language disorders or attention deficit hyperactivity disorder (ADHD); general medical problems, such as epilepsy, gastrointestinal problems or sleep disorders; psychiatric conditions, such as anxiety, obsessive-compulsive disorder or depression; and ASD- associated genetic conditions, including fragile X syndrome and Down syndrome (Lai et al., 2014; Levy et al., 2010; Simonoff et al., 2008; Soke, Maenner, Christensen, Kurzius-Spencer, \& Schieve, 
2018). Using the data from the Autism and Developmental Disabilities Monitoring (ADDM) Network, Levy et al. (2010) found that $83 \%$ of individuals with an ASD diagnosis had as a minimum one concurrent developmental condition, 16\% as a minimum one neurological condition and $10 \%$ as a minimum one psychiatric condition. Boulet, Boyle, and Schieve (2009) reported that $96 \%$ of children with ASD had one or more concurrent developmental conditions. Similarly, Simonoff et al. (2008) found that $70 \%$ of children with ASD had one concurrent developmental, psychiatric or medical condition, whereas $41 \%$ has two or more such conditions. The co-occurring conditions that exist in childhood tend to continue into adolescence. However, some of co-occurring conditions such as depression and epilepsy might appear in either adolescence or adulthood for the first time (Lai et al., 2014). The types of co-occurring conditions, and how they are manifested differ from one individual with ASD to another (Lai et al., 2014; Soke et al., 2018). In general, the higher the number of co-occurring conditions, the greater the developmental disability of the individual (Lai et al., 2014). Hence, co-occurring conditions may contribute to the differentiation among individuals with ASD.

\subsection{Prevalence}

The literature indicates that there has been an increase in the numbers of individuals being diagnosed with ASD in recent years (Centers for Disease Control and Prevention, 2018; Hansen et al., 2015; Lai et al., 2014). Recent results were presented in the USA by the Autism and Developmental Disabilities Monitoring (ADDM) Network, which show that the diagnosis of ASD affected around 1 in 54 children (Maenner et al., 2020). The ADDM also reported that the average prevalence of autism in Asia, Europe and North America is between $1 \%$ and $2 \%$ of the respective population (Centers for Disease Control and Prevention, 2018). In the UK, around 700,000 people have an ASD diagnosis, which is greater than 1 in 100 of the population (National Autistic Society, 2018a). Taking in to account the family of a person with an ASD diagnosis, around 2.8 million individuals live with autism on a daily basis (National Autistic Society, 2017a). No register or accurate statistics are kept in the UK, and any data regarding the probable number of people with ASD in the community is derived from epidemiological surveys (National Autistic Society, 2017a). The 2011 census provides the estimated number of individuals with ASD in England, Scotland, 
Northern Ireland and Wales (see Figure 2-1) (National Autistic Society, 2017a).

Overall, as the prevalence of autism is increasing, this might lead to limited access to both the diagnostic services and those professionals working in the field of ASD.

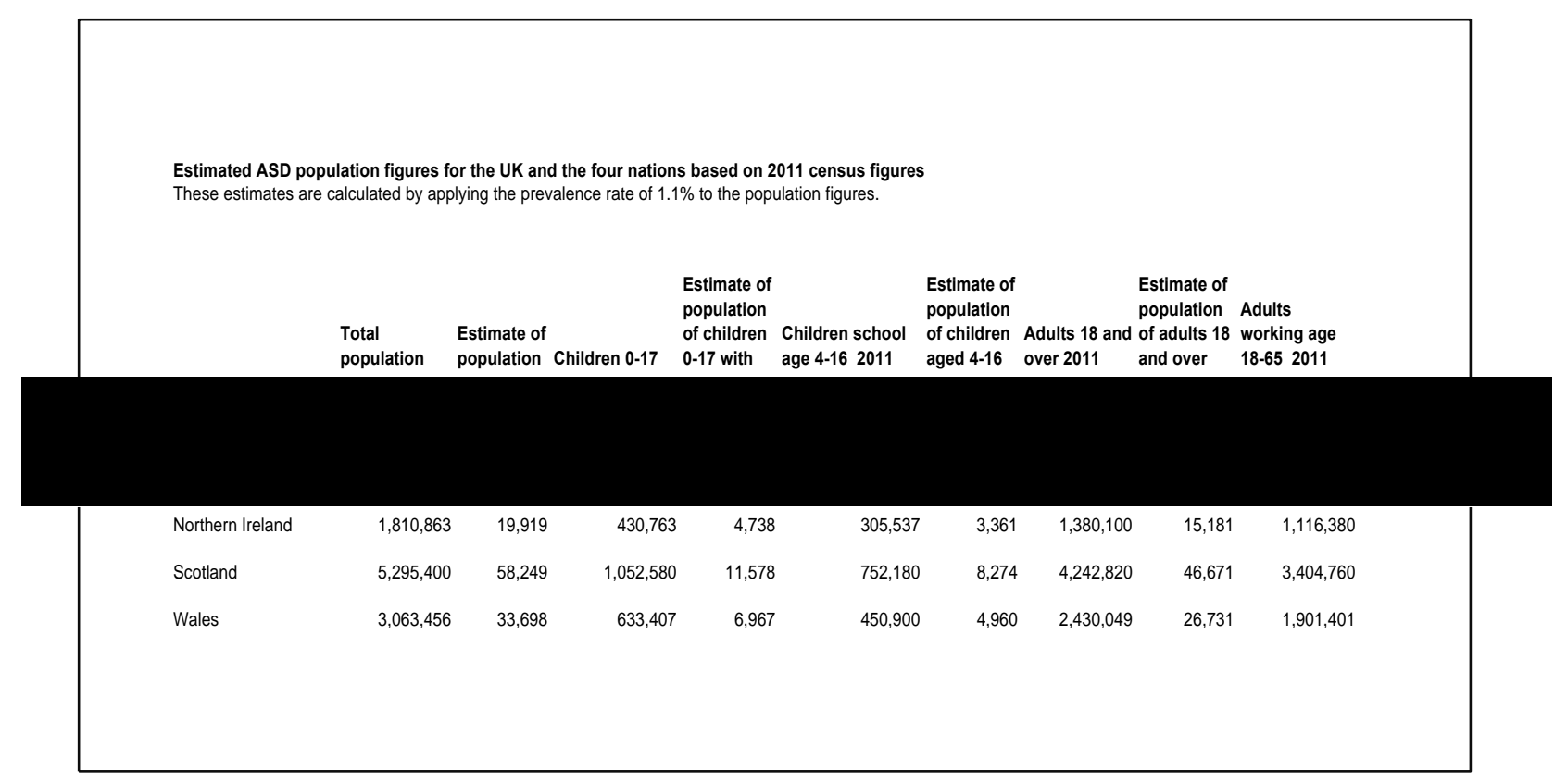

Figure 2-1: Estimated ASD population figures for the UK and the four nations based on 2011 census figures (National Autistic Society, 2017a)

\subsection{Diagnostic process of ASD in the UK}

The precise nature of the diagnostic process of autism differs from region to region in the world (Reed \& Osborne, 2012). Within the UK, the diagnostic process frequently begins when the parent/carer becomes concerned about a child' development, and seeks help from either a GP, a health visitor (for children under 5), another health professional such as a doctor or therapist or a special educational needs co-ordinator (SENCO) at the child's school, depending on where the problem is first identified (National Autistic Society, 2018b; National Health Service, 2019). The professional then typically makes a referral to an autism specialist or a multidisciplinary team to conduct assessment. The multidisciplinary team is comprising a combination of specialists who work together to make an autism assessment. Subsequently, when the assessment is complete, the parents/carers will be given a final report which includes the diagnosis. However, each diagnostic process can differ greatly and could be 
conducted in NHS or in private clinics (National Autistic Society, 2018b; National Health Service, 2019). As this thesis is focusing particularly on the situation in Wales, the diagnostic process of ASD in Wales will be reviewed briefly in the next section.

\subsection{Diagnostic process of ASD in Wales}

The Welsh government has emphasised the fact that professionals in Wales should follow the diagnostic pathway of ASD suggested by the National Institute for Health and Care Excellence (NICE) (National Institute for Health and Care Excellence, 2017; Welsh Government, 2016). NICE is a widely recognised body that provides official advice and information on a variety of different health concerns (National Institute for Health and Care Excellence, 2005). Recently, NICE has created many guidelines that make recommendations concerning the diagnosis and management of autism for children and adults (National Institute for Health and Care Excellence, 2017). As this thesis focuses on parents/carers of children and young people up to 18 years of age, the pathway created by NICE for the identification, referral and diagnostic assessment of individuals under 19s is described briefly below (see Figure 2-2).

\section{Identifying children and young people with probable autism}

If any parents/carers are concerned about the behaviour or development of a child, they should consider the possibility of ASD.

\section{Referring children and young people}

The professional (e.g. health visitor, GP) should refer children younger than 3 years of age to the multidisciplinary autism team when they have developmental delay in either social skills or language. The autism team should involve all relevant professionals, or, if any professionals cannot be part of the team the parents should be able to regularly access those professionals.

- Paediatrician or paediatric Neurologist.

- Child and adolescent psychiatrist.

- Educational psychologist.

- Clinical psychologist.

- Speech and language therapist.

- Occupational therapist. 
While, the professional should refer any children who are, either older than 3 years with deficiencies in language or of any age but with regression in motor skills to a paediatrician or paediatric neurologist, who can then make a referral to the multidisciplinary autism team if required.

\section{Post referral}

After referral to the autism team, at least one of the professionals should consider whether to conduct an ASD diagnostic assessment and/or a different assessment. The paediatrician or paediatric neurologist can also make a referral back to the team if necessary.

\section{Autism diagnostic assessment}

- The autism diagnostic assessment should be begin within 3 months of the referral to the autism team.

- The autism diagnostic assessment should include:

- Detailed inquiries about the concerns of parents/carers as well as the individual's education, social care and home life.

- A developmental history, focusing on features of the child's development and behaviours in accordance with ICD-10 or DSM-IV criteria (at the current time the professionals can use DSM-5 as these NICE guidelines were published in 2011 and DSM-5 published in 2013). The professional may consider using an autism diagnostic instrument to collect this information. This will be discussed more in the following section.

- An assessment of social and communication skills and behaviours acquired through observation of and interaction with child, centering on features in line with ICD-10 or DSM-IV criteria. Professionals might also consider using an autism diagnostic tool to collect this information.

○ A physical examination as well as a medical history, including all previous and existing health conditions

- The professional should use this information, along with their clinical judgment, in order to make a diagnosis based on ICD-10 or DSM-IV criteria. 


\section{Post the diagnostic assessment}

If there is still doubt regarding the diagnosis after the diagnostic assessment, the child should be kept under review.

\section{Medical investigations}

Any medical investigations should not be performed as part of the diagnostic assessment, however based on the individual's personal circumstances and their own clinical judgment should consider the following:

- Genetic tests, if there are particular "dysmorphic features, congenital anomalies and/or evidence of intellectual disability".

- Electroencephalography, if there is a possibility of epilepsy.

\section{Transferring the results after the autism diagnostic assessment}

After the diagnostic assessment, the professional should discuss the findings with the parent/carer without delay, providing them with a report even if the diagnosis is still under consideration. 


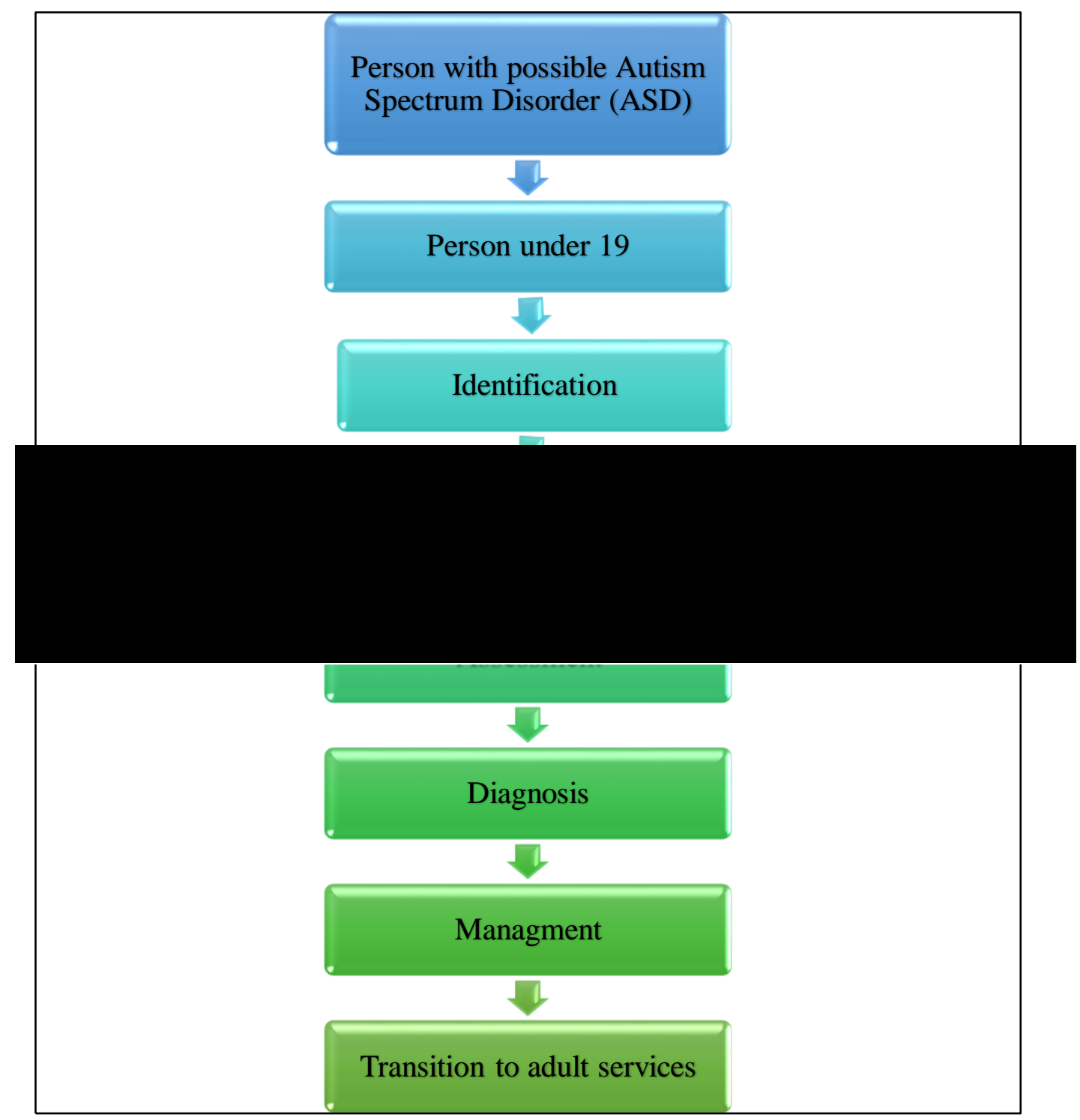

Figure 2-2: Pathway for recognition, referral and diagnostic assessment of possible $A S D$ of individuals under 19 years old (National Institute for Health and Care Excellence, 2017) 


\subsection{Diagnostic instruments of ASD}

Within a diagnostic assessment, a number of autism diagnostic instruments are frequently used to structure the information collected from parents and the individual suspected of having ASD (Charman \& Gotham, 2013). Some instruments used are based on reviews with parents/carers or adult such as the Developmental, Dimensional, and Diagnosis Interview (3di), The Autism Diagnostic InterviewRevised (ADI-R), and The Diagnostic Interview for Social and Communication Disorders (Randall et al., 2018), while others are based on direct observation such as Behaviour Observation Scale for Autism, the Autism Diagnostic Observation Schedule (ADOS) and the Autism Spectrum Disorder-observation for Children (Randall et al., 2018). However, the Autism Diagnostic Interview-Revised (ADI-R) and the Autism Diagnostic Observation Schedule (ADOS) are considered as the "gold standard" tools for ASD diagnosis (Falkmer, Anderson, Falkmer, \& Horlin, 2013), although clinical judgment is still considered vital for a diagnosis of ASD (Falkmer et al., 2013). As emphasised by NICE (2011), clinicians should not rely solely on a specific autism diagnostic tool for an autism diagnosis, but should rather use information from all sources along with their clinical judgment, in order to make a diagnosis of ASD. The ADI-R and the ADOS are reviewed briefly below.

\section{The Autism Diagnostic Interview-Revised (ADI-R)}

The Autism Diagnostic Interview-Revised (ADI-R) (Charman \& Gotham, 2013; Lord, Rutter, \& Le Couteur, 1994; Randall et al., 2018) is a standardised, semistructured interview that is conducted by a clinician with a parent or carer who is aware with the current behaviour and developmental history of the person thought to have ASD. It evaluates behaviour by working through 93 items centering on (a) Communication and Language; (b) Reciprocal Social Interaction; and (c) Restricted and Repetitive, Stereotyped Interests and Behaviours. This diagnostic instrument is suitable for adults as well as children with a mental age of 18 months and above. Conducting and scoring this interview takes around 1.5-3 hours. Scores are allocated on a $0-3$ scale with higher numbers indicating stronger symptoms. 
Cut-off scores are 10 points for the social interaction subscale, 8 points for language and communication if the child is verbal and 7 points if the child is non-verbal, 3 points for restricted and repetitive behaviour (Lord et al., 1994).

\section{The Autism Diagnostic Observation Schedule (ADOS)}

The Autism Diagnostic Observation Schedule (ADOS) (Lord et al., 2000) is a semistructured, standardized observation of the individual referred for ASD assessment. ADOS is appropriate for children and adults with no language, limited language or those who are verbally fluent. A revised version of the ADOS was developed and published in 2012, known as (ADOS-2) (Lord et al., 2012). ADOS-2 includes updated modules 1, 2, 3, and 4 as well as a new module entitled Toddler Module, giving it five modules based on language and development. These models are protocols of activities that take between 30-60 minutes of play-based interaction and/or talking.

- Toddler Module - for children aged between 12 and 30 months of age who do not consistently use speech.

- Module 1 - for children aged 31 months and older who do not consistently use speech.

- Module 2 - for children of any age who can use speech but are not verbally fluent.

- Module 3 - for verbally fluent young adolescents (around 10 to 13 years old) and children.

- Module 4 - for verbally fluent older adolescents (around 14 to 17 years old) and adults.

Scores are assigned on a 0-3 scale, with higher scores indicating higher symptoms. Scores are given directly after completing the module (Charman \& Gotham, 2013). 


\subsection{Difficulties in obtaining a diagnosis}

Several studies have revealed that families with a child with ASD may experience difficulties in accessing autism related services (Kohler, 1999; Ruble, Heflinger, Renfrew, \& Saunders, 2005; Simacek, Dimian, \& McComas, 2017; Thomas et al., 2007). Furthermore, parents/carers of children with ASD may experience more difficulty accessing services when compared with parents/carers of children with developmental disabilities (DDs) (such as cerebral palsy, intellectual disability, or Down syndrome), mental health conditions (MHCs) (such as attention deficit hyperactivity disorder (ADHD), anxiety, or depression) and even more than those parents of children with both DDs and MHCs (Vohra, Madhavan, Sambamoorthi, \& St Peter, 2014).

In respect of parents/carers access to the diagnostic serveries of ASD, research reveals that there is a significant delay of approximately 20 months to 5 years between the first concerns of the parents/carers being noticed and a confirmed diagnosis of ASD and around 3.5 years between them seeking help and a confirmed ASD diagnosis (Crane et al., 2015; Howlin \& Asgharian, 1999; Howlin \& Moore, 1997; Nazneen, Rozga, et al., 2015; Wiggins et al., 2006). This is in spite of the fact that autism symptoms can often be detected as early as 12-18 months of age (Baghdadli, Picot, Pascal, Pry, \& Aussilloux, 2003; Howlin \& Asgharian, 1999; Osterling, Dawson, \& Munson, 2002) with a confirmed diagnosis being given at the age of 30 months (Gillberg, Nordin, \& Ehlers, 1996).

Delay in diagnosis may result in a delay in receiving early intervention services, which are critical for positive outcomes (Matson, 2008; McEachin et al., 1993; Rattazzi, 2014; Siegel, 2008; Smith et al., 2017). Early interventions can take place as early as 2 or 3 years of age, at which time the brain of the child is still developing and more malleable. Treatments undertaken at this time may have a better chance of being effective over a longer period (Dawson et al., 2010; Zwaigenbaum et al., 2015). It has been maintained that identifying autism at an early age and providing early interventions e.g. Applied Behaviour Analysis (ABA), speech therapy, physical therapy and occupational therapy may reduce developmental delays and enhance future learning capabilities, thereby improving the quality of life as a whole (Dugger, 
2012; Nazneen, Matthews et al., 2015). Corsello (2005) found that the age at which a child starts intervention, coupled with the strength of the intervention programme are of paramount importance within the early intervention programmes designed for ASD. This view is supported by Wong and Kwan (2010) when they conducted research on children who were diagnosed early, aged between 17-36 months, and revealed there to be significant improvements in communication, social interaction and language after a therapeutic intervention. Any delay in accessing early intervention services has serious consequences not only for the child, but also the entire family (McMorris, Cox, Hudson, Liu, \& Bebko, 2013). Therefore, the earlier a diagnosis of autism is obtained, the better the long-term outcomes (Wetherby \& Woods, 2006). The long wait times involved in obtaining a diagnosis may also cause stress for the individuals and their families. Previous research has indicated that if parents experience stress during this time, it might lead on to anxiety and depression as well (Hayes \& Watson, 2013; Siklos \& Kerns, 2007).

The delay in obtaining an early diagnosis may be on account of a number of reasons. One possible reason is a shortage of healthcare professionals (Simacek et al., 2017; Thomas et al., 2007). It is well documented that rural communities have particular difficulties accessing autism specialists, resulting in delays in diagnosis (Mandell, Novak, \& Zubritsky, 2005; Thomas et al., 2007) Nevertheless, there are still long waiting lists in urban communities, where there is usually easier access to autistic diagnostic services (Nazneen, Rozga et al., 2015). Furthermore, children with more severe symptoms of ASD, for example, severe speech and language impairments and obvious behavioural deficits, such as toe walking (an inability to make heel-to-ground contact in the first stages of the gait cycle) and hand flapping (moving and raising both hands and rapidly shaking them), could obtain an earlier diagnosis than children with less obvious deficits (Mandell et al., 2005; Siklos \& Kerns, 2007). This might be due to the fact that symptoms of later group less noticeable, and this makes a diagnosis more difficult and delayed (Siklos \& Kerns, 2007). 
Another reason of the delay in receiving a diagnosis might be because of the many specialist appointments and additional evaluation sessions that are often needed to make a diagnosis (Moh \& Magiati, 2012; Siklos \& Kerns, 2007; Wiggins et al., 2006; Wiggins, Baio, \& Rice, 2006b). The requirement to meet with a variety of professionals for several times in different locations can also be very stressful for those individuals who might later be diagnosed with autism (Crane et al., 2015). People with ASD are very sensitive to changes in their situations and excessively reliant on routines (American Psychiatric Association, 2013a). Some families have to travel long distances to access these services (Oberleitner et al., 2007). This is a cost not only to the family but also to the specialist healthcare teams who have to travel to reach different areas (Reese, Braun, et al., 2015). Recent research in the UK found that healthcare and educational systems work independently from each other with little crossover of activity (Hurt et al., 2019). Therefore, it could prove be difficult to get multi-disciplinary teams together, especially when they span different disciplines.

Moreover, the "Wait-and-See" approach that is often taken by primary healthcare providers (Klin, Klaiman, \& Jones, 2015; Siklos \& Kerns, 2007), an absence of knowledge regarding autism symptoms, uncertainty about the correct diagnosis and an insufficient awareness of the significance of early diagnosis might also be seen as reasons for delayed diagnosis (Book, 2009; Siklos \& Kerns, 2007).

The delay in receiving an early diagnosis of ASD could occur because of any of the aforementioned reasons. Still, this substantial delay should be considered to be an issue that may increase stress for the individuals and their families, as well as obstructing early interventions, which have been proven to have positive effects on long-term outcomes. The following section will consider the degree of parental satisfaction with the diagnostic process of ASD, and the factors associated with this. 


\subsection{Parental satisfaction with the experience of receiving an ASD diagnosis and associated factors}

Research has shown that undergoing a positive experience when obtaining an ASD diagnosis has a considerable effect on the initial reaction of parents toward a developmental disability diagnosis (Leff \& Walizer, 1992; Woolley, Stein, Forrest, \& Baum, 1989). Despite this, the overall process of receiving a diagnosis of ASD has often been identified as unsatisfactory for parents (e.g. Crane et al., 2015; GoinKochel, Mackintosh, \& Myers, 2006; Howlin \& Moore, 1997; Potter, 2017; Siklos \& Kerns, 2007) for which there could be several factors.

One consistent factor reported by parents/carers is the long delay between seeking help and receiving a confirmed diagnosis (Crane et al., 2015; Howlin \& Moore, 1997; Siklos \& Kerns, 2007). For example, a study of around 1000 UK parents (Crane et al., 2015) found there to be an average 3.5 years between an initial appointment with a health professional to a confirmed diagnosis of autism. Parents who received a diagnosis later were more dissatisfied with the diagnostic process. Somewhat surprisingly, this diagnostic delay has not decreased greatly since the previous largescale UK survey of almost 1300 parents of children with autism (Howlin \& Moore, 1997). This study found that children were diagnosed on average at 6.11 years of age, although parents noticed concerns about their child's development and behaviour on average at 1.69 years of age and sought help on average at 2.3 years of age indicating a delay of 3.81 years since parents sought professional help. Similarly, in a small-scale Canadian study of fifty-six parents of children with autism (Siklos \& Kerns, 2007) the average age of the child when parents first sought help was 2 years of age and the average age when final diagnosis was received was 5 years, thereby indicating that waited around 3 years to obtain a diagnosis. Even though some research has found that the age of the child at time of diagnosis earlier than other reported results, the average delay between parents seeking help and receiving a diagnosis was still around 2 years (Chamak, Bonniau, Oudaya, \& Ehrenberg, 2011; Goin-Kochel et al., 2006). Indeed, the level of parental dissatisfaction with the diagnostic process is still high, irrespective of some suggestions that it is taking less time overall to obtain a final diagnosis. Therefore, a delay in obtaining a diagnosis could lead to increased dissatisfaction on the part of the parents/carers. 
Moreover, it has been found that parents/carers were particularly dissatisfied when they had to consult several professionals in order to receive the diagnosis. For example, Goin-Kochel et al. (2006) conducted a study based on an online survey of 494 parents of children with ASD from five countries. The greatest number of parents was from the US ( $n=377,76.3 \%)$, followed by parents from England/Ireland ( $n=32$, $6.5 \%)$, Canada $(n=30,6.1 \%)$, Australia/New Zealand $(n=18,3.6 \%)$, and other, unlisted places $(n=6,1.2 \%)$. Parents reported that they had to visit an average of between four and five professionals before receiving a diagnosis (range 1 to 29). Although, seven parents $(1.4 \%)$ reported that there were "too many to count". This same research found that by the time parents had seen several professionals, the child was older receiving the diagnosis which resulted in parental dissatisfaction with the diagnostic process. Siklos and Kerns (2007) reported that parents visited an average of 4.46 (range 2-14) professionals throughout the ASD diagnostic process; 23\% of parents visited two professionals, $14 \%$ visited three professionals, $21 \%$ visited four professionals, $41 \%$ visited more than five professionals and 5\% visited more than 10 professionals before receiving a confirmed diagnosis of ASD all of which led to parental dissatisfaction. Therefore, the number of professionals seen during the diagnostic process may contribute to making the overall diagnostic process a more negative experience.

The period following diagnosis is mainly one of seeking appropriate help and support (Mansell \& Morris, 2004). Professional help and support after a final diagnosis is very important to parents, such as arranging a referral to a local service, arranging a follow up appointment with a speech and language therapist, establishing contact with other parents, offering personal support/counselling and contact with a charity (Crane et al., 2015; Mansell \& Morris, 2004; Siklos \& Kerns, 2007). However, parents also continue to express dissatisfaction with the overall diagnostic process because of their dissatisfaction with the help and support they have been provided after the confirmed diagnosis of their child. It has been found that parents of children with ASD are less satisfied with the support services offered after receiving diagnosis than the parents of children with other developmental disorders such as Down syndrome (Siklos \& Kerns, 2006). Crane et al. (2015) found that parental satisfaction with post diagnostic support was a strong predictor of the satisfaction with the ASD diagnostic process as whole. This research reported that $61 \%$ of their sample was dissatisfied with the post- 
diagnostic help offered as they were not given enough information, an even greater number than the 35\% suggested by Howlin and Moore (1997) and the 53\% suggested by Siklos and Kerns (2007). The Crane et al. (2015) study suggested that parents, particularly those who had had a good experience of the diagnostic process, might well have higher expectations of the level of care offered post diagnosis.

A study by Crane et al, (2015) found that less than a quarter of parents were offered help and support directly after diagnosis (e.g. arranging an appointment with occupational therapist) and around $40 \%$ of parents were not provided any postdiagnostic support in any way. Out of a study of 306 fathers of children with ASD who were living in the UK (Potter, 2017) 184 (60\% of the total) replied to the open-ended question related to perceptions on the diagnostic process. More than a fifth of those fathers $(n=39)$ referred to substantial absence of post-diagnostic services. One of the participants shared the fact that following his son's diagnosis, he just received a leaflet. He then chose to pay to obtain private help and support services. Another participant stated that he received the final diagnosis without any advice regarding where to go for help and support, making the first months after diagnosis very difficult. Clearly, post-diagnostic help and support may associate with parents/carers satisfaction with the overall diagnostic process.

Furthermore, it has been indicated that the stress of the diagnostic process is one of the factors that associated with parental dissatisfaction overall (Crane et al., 2015; Moh \& Magiati, 2012; Siklos \& Kerns, 2007). The process of receiving an ASD diagnosis is very stressful for parents, with the possibility that it could lead to anxiety and depression (Hayes \& Watson, 2013). However, it has been noted that stress levels for parents of those children with greater communication deficiencies are reportedly lower, maybe indicating a greater degree of parental satisfaction with overall diagnostic process (Siklos \& Kerns, 2007). This is possibly because of the fact that in such cases, ASD symptoms are more obvious to parents from the outset (e.g. language fails to develop), clearly indicting that there is an obvious issue with their child's development which then means a swifter diagnosis overall (Crane et al., 2015; Howlin \& Moore, 1997; Siklos \& Kerns, 2007). Crane et al. (2015) reported that a high proportion (84\%) of their sample found the process of obtaining an ASD diagnosis to 
be stressful, an even higher figure than the $82 \%$ reported by Siklos and Kerns (2007). Many of the parents cited that the long wait times for examining and diagnosis as being the main reason of their stress because they were wondering what might be wrong. Parental stress around the diagnostic process may clearly lead to lower levels of satisfaction with the process overall.

It has also become apparent that when families were provided with information at diagnosis regarding the nature of autism, were directed towards places where they might obtain support and given some indication as to how autism could potentially impact the life of the child, they reported greater levels of satisfaction with the diagnostic process (Crane et al., 2015; Osborne \& Reed, 2008). During 15 focus group discussions that were conducted with the parents of children with ASD at a variety of locations throughout England (Osborne \& Reed, 2008), a high percentage (87\%) of parents revealed that they had not been provided with any information about ASD and they did not know what it meant or how it was.

These parents also requested that training be given to professionals, specifically regarding the information they should provide to parents of children diagnosed with ASD and that information be provided to them immediately and completely upon receiving a diagnosis (Osborne \& Reed, 2008).

If clear, comprehensive information from the correct sources were to be offered at the time of diagnosis, the parents would be less likely to listen to misinformation offered by others. Hasnat and Graves (2000) also found that parents were more likely to feel satisfied if they were given a great amount of information, even if they did not ultimately use it all:

"I don't think it's the responsibility of the paediatrician or neurologist to judge what parents can and can't handle. If I'm sitting there and saying 'give it to me" and I cry when they tell me, that's my problem . . it should be up to the parents to say how much information they want and it should be freely given." (Hasnat \& Graves, 2000, p. 34)

More satisfaction with the information provided at diagnosis might well contribute to a more positive experience overall. 
In addition, it has been found that parental satisfaction with the diagnostic process was highest amongst those who had higher levels of education. Going-Kochel et al. (2006) found that level parental education was associated with obtaining an earlier diagnosis, resulting in greater satisfaction with the overall diagnostic process. Going-Kochel et al. (2006) emphasised that professionals have to make every possible effort in order to ensure that pathways of diagnosis are equitable for all parents. Those parents with lower levels of education might well have less opportunities to obtain information and support, and could be less able to explain their concerns about their child development. It has also been indicated that levels of satisfaction with the overall diagnostic process were highest amongst those parents whose children obtained a diagnosis before school ages (Goin-Kochel et al., 2006; Howlin \& Moore, 1997). This might well be related to the fact that a great number of these children were diagnosed with 'classic' autism. Children with what was called 'high functioning autism or Asperger syndrome' typically receive a diagnosis at a later age, because signs such as repetitive patterns of behaviour and impaired social interaction generally manifest more clearly later in life (Howlin \& Asgharian, 1999; Hyman, Rodier, \& Davidson, 2001). The parents also reported that a longer delay could increase frustration, resulting in a negative experience of the overall diagnostic process (Howlin \& Asgharian, 1999). Therefore, a child's age at time of diagnosis may be associated with parental satisfaction with the overall ASD diagnostic process.

To sum up, the previously reviewed studies have revealed that most of parents of children with ASD were not satisfied with the overall diagnostic process. Although some research has addressed possible factors for this (such as time taken to get diagnosis, age of child at diagnosis, information given at diagnosis, post-diagnostic support, stress of the diagnostic process, number of professionals involved, parent's education level), understanding of the factors is still emerging. Furthermore, the majority of the studies reviewed above have typically examined these factors independently. To the researcher's knowledge, no study has been conducted in Wales which aims to explore the parents/carers experience of the ASD diagnostic process as well as the associated variables. Such study would be particularly relevant following the implementation of the Refreshed Autistic Spectrum Disorder Strategic Action Plan 2016 that was developed by Welsh Government (Welsh Government, 2016). 
As cited previously, this plan aimed to offer improvements in the assessment and diagnostic services of children, by creating a national standardised assessment pathway, thereby ensuring consistent provision for all children. In addition, to improve the access time for children and young people, a target of 26 weeks waiting time was set, from initial referral to first assessment appointment (Welsh Government, 2016). During 2012-2013 a nationwide survey was carried out across the UK (Crane et al., 2015 ), but only $3 \%$ of the respondents were from Wales. This previous study also was not able to ask the parents if they had more than one child with ASD. If they had, the diagnostic process for youngest child could have been more positive. Therefore, in view of these recent survey limitations and supposed improvements in the ASD diagnostic services in Wales brought about by the Refreshed Autistic Spectrum Disorder Strategic Action Plan, it was timely to conduct a study of parental experiences around obtaining an ASD diagnosis in Wales. Their experience with the diagnostic process will be explored and also whether children are being diagnosed earlier, thereby increasing the level of parental satisfaction. The study will also examine the factors associated with parental satisfaction with the overall diagnostic process.

There seems to be considerable evidence to indicate that parents of children with ASD face difficulties when accessing diagnostic services. The fact that there is inevitably a long delay between the parent seeking help from professionals and receiving a confirmed diagnosis of ASD and most of the parents of children with ASD are dissatisfied with the diagnostic process. Many rural communities lack access to health care professionals with autism specific expertise that may hinder timely ASD diagnosis (Mandell, Novak, \& Zubritsky, 2005; Thomas et al., 2007). Research has indicated that children living in rural and underserved areas are diagnosed at a later age than those living in urban areas (Wiggins, Baio, \& Rice, 2006). Delays in diagnosis may also result in delayed access to early intervention services which are crucial for improvement and achieving successful outcomes. As a result, families from rural areas who are concerned about their child's development frequently travel long distances for an evaluation, incurring additional travel costs and receiving recommendations from healthcare professionlas unfamiliar with resources in the families' communities (Reese et al., 2013). 
There is clearly a pressing need for a solution which can support parents and children who are in need of such services. Information Systems and Communication Technologies (ICTs) for example the Internet, computers and mobile phones, could address some of health care related challenges currently faced in both developed and developing countries by delivering high quality, accessible, and effective services (World Health Organisation, 2010). Telehealth uses communication technologies to facilitate communication between health professionals and patients, as well as between health professionals at distance. This may increase access to health care services and overcome geographical barriers (World Health Organisation, 2010). Therefore, telehealth might be used to improve access to the ASD diagnostic services and also the parent/carer experience with the diagnostic process as a whole. In particular, telehealth might be used as a potential solution to support diagnostic assessment of ASD and reduce the time taken to obtain a diagnosis (Reese, Braun et al., 2015; Reese, Jamison et al., 2015; Smith et al., 2017).

Telehealth may give an opportunity to reach families in rural and underserved areas, and even families in urban areas, where the diagnostic services are often impacted by long waiting lists, because of the shortage of autism-related professionals (Nazneen, Rozga et al., 2015). There is also growing evidence to suggest that telehealth approaches could decrease costs for both providers and patients (e.g. travel time, transportation expenses, work absence) and increase coverage area to providers (Dunn, Choi, Almagro, Recla, \& Davis, 2000; Gros et al., 2013; Trott \& Blignault, 1998). By offering families the opportunity to play a significant role in the development of their child, telehealth technology could expedite both the diagnostic process and early intervention services (Nazneen, Matthew et al., 2015; Wainer \& Ingersoll, 2015).

Telehealth technology may be most useful to be used during the early stages of the ASD diagnostic process, specifically with the diagnostic assessment. Remote diagnostic assessment can take place by connecting clinicians with individuals with ASD and their families, whilst they remain in their regional clinics or homes (Reese, Jamison et al., 2015). Telehealth enables different professionals involved in a diagnosis to meet with the family in real time in order to assess the child, thereby tackling the issues associated with geographical spread. It also allows videos of the child's behaviours and interactions to be uploaded, so that the clinicians can make an 
assessment remotely and share it with parents/carers or other health professionals (Nazneen, Rozga et al., 2015). The fact that health professionals can conduct remote observations in the family home, eliminates the need for the family to travel and means that observations can take place in more naturalistic settings (Reese, Jamison et al., 2015). Consequently, the diagnostic process can be improved and the time taken to get a diagnosis of ASD accelerated (Smith et al., 2017). This will be reviewed in next chapter (Study1), to better understand how telehealth has been used for diagnostic assessment, which telehealth approaches are used in the diagnosis of ASD, whether they are acceptable and feasible and how they compare with more traditional face to face methods. The following section describes the definitions of telehealth, the various types of telehealth and communication technologies, and how it can be used within the field of ASD and other medical conditions.

\subsection{Telehealth definitions}

There is no common definition of telehealth which is used across all literature (Fatehi \& Wootton, 2012; Sood et al., 2007). The terms 'telemedicine' and 'telehealth' are often used interchangeably (American Telemedicine Association, 2017; Fatehi \& Wootton, 2012; World Health Organisation, 2010). However, some studies consider telemedicine and telehealth to be different terms, which can have different meanings. For instance, Katharaki (2006) identified 'telemedicine' as "the use of electronic medical information and communication to provide and support healthcare when distance separates the participants" (p. 353). Bashshur, Bashshur, Shannon and Sanders (1997) suggest a definition of 'telemedicine' that involves communication only between physicians and patients: telemedicine is about use of information and biomedicine technology in order to help, enhance, or assist collaboration and delivery of healthcare related services between physicians and patients at distance locations.

It has been considered that 'telehealth' is a broader, more general term than 'telemedicine' term. For example, Lovell, Redmond, Basilakis, Shany and Celler (2010) highlighted that "Telehealth incorporates a wider range of health-related activities, including patient and provider education, point-of care diagnostics, clinical decision support services, and most importantly, provides tools for self-management 
of disease and wellness" (p. 5267). This is in contrast to 'telemedicine', which they defined it as "a system of healthcare delivery in which physicians examine patients through the use of telecommunications technologies" (Lovell et al., 2010, p. 5267). Similarly, Koch (2006) described 'telemedicine' as 'the use of audio, video and other telecommunications and electronic information processing technologies for the transmission of information relevant to the diagnosis and treatment of medical conditions, or to provide health services or aid healthcare personnel at distant sites" (p. 566). He also considered that 'telemedicine' part of 'telehealth' that has 'broader scope towards health promotion and disease prevention" (Koch, 2006, p. 566).

However, as revealed previously, the terms 'telemedicine' and 'telehealth' are often used interchangeably. The World Health Organisation (WHO) considers the terms telehealth and telemedicine to be synonymous (World Health Organisation, 2010). WHO defines telehealth in the following way:

The delivery of health care services, where distance is a critical factor, by all health care professionals using information and communication technologies for the exchange of valid information for diagnosis, treatment and prevention of disease and injuries, research and evaluation, and for the continuing education of health care providers, all in the interests of advancing the health of individuals and their communities (World Health Organisation, 2010, p. 9).

Similarly, the American Telemedicine Association (ATA) considers 'telemedicine' and 'telehealth' to be the same thing. The ATA defined 'telemedicine' as "the remote delivery of health care services and clinical information using telecommunications technology. This includes a wide array of clinical services using internet, wireless, satellite and telephone media" (American Telemedicine Association, 2017, para 1). Following the example of WHO and ATA, the terms 'telemedicine' and 'telehealth' were used interchangeably throughout the searching process, and 'telehealth' term was used across the thesis in order to have consistency amongst the included chapters. 


\subsection{Communication technologies}

Telehealth includes diverse forms of information transmission (such as sound, voice, text, picture, and video) and user interfaces (such as desktop computers, laptop computers, personal digital assistants, fax machines, telephones, and mobile/smart phones) (Miller, 2007). Telehealth also includes communication technologies that can be classified into two groups, namely Real-Time and Store-and-Forward (Maheu, Whitten, \& Allen, 2001). Real-Time technologies are also known as Synchronous technologies. Real-Time technologies allow simultaneous interaction and discussion, either between patient and healthcare provider or between two or more healthcare providers (Maheu et al., 2001). The telephone is considered to be the most common type of Real-Time technology, yet is often ignored when discussing technology, in spite of its many benefits for both patients and healthcare providers (Maheu et al., 2001). Additionally, Internet audio services have become widespread methods of synchronous communication technology, as a means of avoiding the high cost of regular telephone services. Interactive tele-videoconferencing (ITV) is considered to be the main form of synchronous communication for telehealth programmes. Using ITV, two or more individuals can see and hear each other and can even remotely share documents in real time, by using video equipment (Maheu et al., 2001).

In contrast, Store-and-Forward technologies (Asynchronous technologies) do not depend on both the sender and the receiver (e.g. healthcare provider and patient) being presented at the same time (Maheu et al., 2001). Store-and-Forward technologies allow health information to be gathered and sent to another party, who can retrieve it whenever suitable (Maheu et al., 2001). For example, a health professional could use electronic mail to send information to a patient or to another health professional. 


\subsection{Types of telehealth}

Khan, Qurashi and Hayee (2007) and Norrise (2002) have devised a clear classification of telehealth that involves four different types: Tele-Consultation, TeleEducation, Tele-Monitoring and Tele-Surgery.

\subsubsection{Tele-Consultation}

The medical consultation is vital within clinical practice. The use of teleconsultation to support clinical decision making is therefore considered to be the most common type of the telehealth. The patient is able to consult the health professionals who are located elsewhere. This can improve access to the healthcare services as well as reducing waiting times.

Tele-consultation could be done in two ways:

- Real-Time Tele-consultation: this might be conducted between two or more health care providers with/ or without the patient, in real time.

- Store-and-Forward Tele-consultation: the information related to the patients is saved and then transferred electronically to the healthcare professional or team of professionals, for them to provide the consultation.

\subsubsection{Tele-Education}

A wide range of information can be provided online via the Internet. Any information that is related to healthcare or medicine can be considered as a recourse for teleeducation. The health-related sources might be used to educate healthcare providers as well as healthcare/medical students. These sources can offer educational material at a distance, particularly for those living in rural areas, and along with the benefits of being low cost and easy to access. There are various types of tele-education depending on who is receiving the information and what the ultimate goal is:

- Clinical education from tele-consultation;

- Clinical education through the use of Internet;

- Academic study through the use of Internet;

- Public education through the use of Internet. 


\subsubsection{Tele-Monitoring}

Tele-monitoring is identified as being the use of telecommunication technologies to collect repeated or routine information on a patient's condition. The process might be manual with the patient recording the data and sending it by telephone or computer. Alternatively, the process could be completely automated. Tele-monitoring is crucial in order to carry out continued observation on the health of the patient and can help in deciding if any changes in treatment or prescription are necessary. It has been reported that tele-monitoring has the potential to improve the conditions of home based patients with illnesses such as diabetes (Lee, Greenfield, \& Pappas, 2018). Furthermore, it could also be successful for those with heart conditions (Hsieh, Li, \& Yang, 2013). For instance, Electrocardiograph (ECG) readings can be transmitted by elderly patients at home.

\subsubsection{Tele-Surgery}

Tele-Surgery refers to surgery being conducted at a distance using telecommunication technologies. There are two main areas of tele-surgery, namely tele-monitoring, when a specialist remotely supports the surgeon who is actually doing the surgery via audio and video interaction. Another area is called tele-presence surgery, when the surgeon can direct the robotic arms to perform the surgery at a remote location.

\subsection{Use of telehealth with clinical specialties and ASD condition}

The following sections discuss the use of telehealth within different clinical specialties, including ASD.

\subsubsection{Use of telehealth within clinical specialties}

Research indicates that telehealth has the potential to be used within a variety different specialties, giving positive results. For example, Store-and-Forward technology enables healthcare professionals to send digitized medical images, such as X-rays and Computerised Tomography (CT) scans to another healthcare professional at remote location (Al-Safadi, 2016). It has been indicated that using tele-radiology has the potential to facilitate clinical decision making regarding the patient's diagnosis 
thereby improving patient care (Al-Safadi, 2016; Kammerer, Hammon, Schlechtweg, Uder, \& Schwab, 2015).

Telehealth can also be used to provide remote interactive monitoring services to people living with diabetes (Guljas, Ahmed, Chang, \& Whitlock, 2014; Lee et al., 2018). Telehealth is a cost-effective and beneficial way of reducing the follow-up visits necessary for diabetes management (Montani, Bellazzi, Quaglini, \& D'Annunzio, 2001). Diabetes is treated by keeping glycaemic control, as measured by glycosylated haemoglobin (HbA1c) (Inzucchi et al., 2012) although it is challenging for many patients to maintain the required levels (Stone et al., 2013). Research suggests that telehealth might be more effective at ensuring glycaemic control than typical care (Steventon, Bardsley, Doll, Tuckey, \& Newman, 2014) with the patient regularly sending physiological information, including blood glucose, to a healthcare professional working at a remote location who can then immediately send feedback (Steventon et al., 2014). Lee et al.'s (2018) systematic review of systematic reviews of randomised controlled trials indicated that telehealth intervention made significant improvement in glycosylated haemoglobin (HbA1c) levels, when compared with typical care received. Results such as this could mean fewer complications, as well as fewer expensive unplanned admissions to the hospital (Steventon et al., 2014).

Furthermore, research consistently reports that the use of telehealth might be useful in the distance diagnosis and monitoring of patients with cardiac diseases, known as telecardiology (Brunetti et al., 2015). A review of various studies has indicated that electrocardiograph (ECG), echocardiography $(\mathrm{ECHO})$ and other related images can be transferred to the cardiologist at a distance, who can read the data and give feedback to the patient (Hsieh et al., 2013). This could take place before the patient enters hospital, detecting the problem whilst they are still in the ambulance; in-hospital (Teleconsultation); when transferring patients' data from a small hospital to the cardiologist at a remote location so that he can interpret and form a diagnosis; post-hospital (Home monitoring); enabling the patient to transmit data (e.g. ECG data) to the hospital by using technologies such as a mobile phone, so the cardiologist can review the data and give feedback. A recent systematic review also found that tele-cardiology is associated with an improvement in health outcomes and reduction in hospitalisations, readmissions and mortality (Kruse, Soma, Pulluri, Nemali, \& Brooks, 2017). 
Telehealth might also be used to deliver mental health services remotely, including diagnosis, assessment, symptom tracking, and treatment for individuals with mental disorders such as depression, Post-Traumatic Stress Disorder (PTSD), substance abuse, dementia and panic disorder (Aboujaoude, Salame, \& Naim, 2015; Langarizadeh et al., 2017). A review by Hilty et al. (2013) found that telemental has the potential to provide diagnosis and assessment for many different population, including adults, children and elderly, in a way which is quite similar to the traditional face to face approach. Telemental is considered to be an essential part of telehealth development and may advance faster than other medical and/or healthcare specialties (Aboujaoude et al., 2015) possibly due to the "hands off" services generally related to mental health, along with the fact that investigative tools such as physical examination, radio-imaging and lab tests are generally less required (Aboujaoude et al., 2015).

Overall, telehealth has the potential to be used with many different health/clinical specialties, offering positive results for both adults and children. On account of the apparent benefits of such systems, there has been mounting interest in the use of telehealth in ASD (Aresti-Bartolome \& Garcia-Zapirain, 2014). A systematic review has reported that telehealth was not included within the field of ASD before 2004 (Aresti-Bartolome \& Garcia-Zapirain, 2014). So, it is still in its infancy but is growing steadily. Telehealth approaches have recently been explored as a way of supporting the delivery of a range of services for people with ASD and their families. A systematic review conducted by Boisvert et al. (2010) revealed that telehealth has a promise to be a feasible means to address the need for improved access to services for autistic individuals. The following section summarises the previous use of telehealth within the field of ASD. 


\subsubsection{Use of Telehealth with ASD}

Children with ASD may show challenging behaviours, such as aggression and selfinjury (Oliver, Petty, Ruddick, \& Bacarese-Hamilton, 2012). It has been proven that a treatment approach including two steps, firstly the conducting of a functional analysis (FA) followed by a functional communication training (FCT) is effective in reducing problem behaviour in children (Tiger, Hanley, \& Bruzek, 2008; Wacker et al., 1998). FCT is classified as behavioural and evidence based intervention that replaces unsuitable behaviours, such as self-injurious and aggressive behaviours with alternative communicative responses (Carr \& Durand, 1985). FA is conducted to inform planning of intervention for FCT, by detecting environmental events that acts as reinforcers of challenging behaviour (Carr \& Durand, 1985). FA, in addition to FCT, is well documented as an effective means of intervention to reduce challenging behaviour amongst people with disabilities, such as ASD (Tiger et al., 2008). However, many families experience difficulties in accessing behaviour analysis services (Wacker, Lee, Dalmau, Kopelman, Lindgren, Kuhle, Pelzel, Dyson, et al., 2013; Wacker, Lee, Dalmau, Kopelman, Lindgren, Kuhle, Pelzel, \& Waldron, 2013).

Burgeoning research suggests that telehealth could be used to improve individual access to such services. Several studies have used telehealth to coach parents of children with ASD how to conduct behavioural assessments, such as FA and FCT, via the use of videoconferencing, with parents and child located at either regional clinic, home or school (Lindgren et al., 2016; Machalicek et al., 2016; Simacek et al., 2017; Suess et al., 2014; Suess, Wacker, Schwartz, Lustig, \& Detrick, 2016; Wacker, Lee, Dalmau, Kopelman, Lindgren, Kuhle, Pelzel, Dyson, et al., 2013). These studies have found that when a behaviour analyst can offer remote consultations, parents can successfully use telehealth to conduct FA and FCT assessments, leading to a reduction in problem behaviour. Wacker, Lee, Dalmau, Kopelman, Lindgren, Kuhle, Pelzel, Dyson, et al. (2013) found the average reduction in problem behaviour to be $93.5 \%$. Similarly, Suess et al. (2016) identified that problem behaviour was decreased by an average of $65.1 \%$ and Lindgren (2016) found the mean percentage reduction in problem behaviour to be greater than $90 \%$. Therefore, telehealth shows much promise for conducting remote behavioural and functional assessments. 
Telehealth has also been used effectively in the delivery of parent mediated interventions. Such interventions intend to increase parental knowledge and the use of ASD behavioural intervention strategies with children in their daily life. For example, some studies focus on the use of telehealth to deliver the parent curriculum of the Early Start Denver Model (ESDM), an early behavioural intervention programme for toddler and preschool-aged children with ASD (Vismara et al., 2013; Vismara, Young, \& Rogers, 2012). Web-based instructional content, in combination with weekly videoconferencing coaching sessions or only weekly video-conferencing coaching sessions were identified as potential ways in which to deliver parent training. Results indicated that parents were able to use the intervention strategies with dependability. In addition, their children demonstrated improvements in social communicative behaviours (e.g., language, imitative behaviours). Parents also indicated that the website was an important source of information, to support what they had learned during the weekly session. However, they still believed sessions with therapists were crucial for understanding how to use the interventions with their children on a daily basis (Vismara et al., 2013; Vismara, Young, \& Rogers, 2012).

Similarly, some studies examined the use of ImPACT telehealth programmes, intended to teach parents to support the social communication of their children within the environment of play and day-to-day routines (Ingersoll \& Berger, 2015; Brooke Ingersoll, Wainer, Berger, Pickard, \& Bonter, 2016; Pickard et al., 2016). Either webbased lessons alone, or in combination with weekly videoconferencing coaching sessions delivered by a therapist were identified as a potential means for delivering parent training. Results suggested that children improved their social communication skills. Furthermore, parents improved their use of the intervention practices and indicated that telehealth as service delivery model was useable, although who received therapist assistance saw greater improvements.

Overall, although literature regarding the use of telehealth in this area is limited and still in its infancy, studies exploring whether telehealth can improve access to and delivery of behavioural interventions for autistic people, have been promising. Therefore, the use of telehealth as a service delivery model to coach parents/carers how to conduct behavioural and functional assessments, has the potential to be both achievable and effective. In addition, the use of telehealth has the potential to support 
parents/carers to fully understand, as well make use of early intervention practices, during their daily interactions with their children. Because of this, telehealth might well be used as means of improving access to ASD diagnostic services for parents/carers and warrants further investigation.

Telehealth has started to be used in remotely conducting the diagnostic assessment of ASD in the United States (Reese, Jamison et al., 2015; Smith et al., 2017). This gives an opportunity to reach families in rural and underserved areas, and even families in urban areas, where the diagnostic services are often impacted by long waiting lists, because of the shortage of autism-related professionals (Nazneen, Rozga et al., 2015). Remote diagnostic assessment can take place by connecting clinicians with individuals with ASD and their families, whilst they remain in their regional clinics or homes (Reese, Jamison et al., 2015). Consequently, the diagnostic process might be improved and the time taken to get a diagnosis of ASD could be accelerated (Smith et al., 2017). This will be reviewed in next chapter (Study1), in order to better understand how telehealth has been used for diagnostic assessment, which telehealth approaches are used in the diagnosis of ASD, whether they are acceptable and feasible, and how they compare with more traditional face to face methods.

\subsection{Summary}

This chapter began by exploring the background of ASD in terms of definitions, diagnostic criteria, co-occurring conditions, diagnostic process in the UK (particularly in Wales) and the diagnostic tools used for collecting information within a diagnostic assessment. Then, it presented the difficulties that are faced by individuals and families in obtaining a diagnosis. Satisfaction of parents/carers with the diagnostic process and its associated factors was then reviewed. Overall, there seems to be some evidence to indicate that the parents of children with ASD face difficulties in accessing diagnostic services, particularly the long delay between seeking help from professionals and receiving a confirmed diagnosis of ASD. Unsurprisingly, most of the parents of children with ASD are dissatisfied with the diagnostic process. 
One possible solution to this problem is to use telehealth in order to support diagnostic assessment regardless of geographic location. This might well reduce the time it takes to get a diagnosis. Background information about telehealth with definitions, communication technologies and telehealth types was then provided. The use of telehealth within clinical specialities other than autism was also highlighted. From this, it was found that as telehealth has the potential to be used within many clinical specialities and used to improve access to and the delivery of behavioural interventions for autistic people, with positive results, it could particularly be used to support the diagnostic assessment. The following chapter (Study1) will present a scoping review that examines the use of telehealth methods in the remote diagnostic assessment of ASD. 


\section{Chapter 3: (Study1) Use of Telehealth for Facilitating the Diagnostic Assessment of Autism Spectrum Disorder (ASD): A Scoping Review.}

\subsection{Introduction}

This chapter presents a scoping review that examines the use of telehealth approaches in the diagnostic assessment of Autism Spectrum Disorder (ASD). The chapter begins by defining ASD and scoping its global prevalence. Some of the issues/challenges associated with current systems of diagnosis of ASD are also presented with highlighting the importance of timely diagnosis and early intervention. The importance of telehealth, along with its associated advantages and challenges are presented briefly, before introducing the objectives and methodology that were employed in this review. Key findings of the review are then discussed ending with the conclusion and summary.

\subsection{Context}

Autism Spectrum Disorder (ASD) is a developmental condition characterised by impairment in terms of social communication and social interaction and a repetitive and restricted pattern of interest, behaviour and activity (American Psychiatric Association, 2013b). Around 1 in 54 children in the United States has been diagnosed with ASD (Maenner et al., 2020) (for more information see Section1.2). With regard to the diagnostic criteria, the latest revision of the Diagnostic and Statistical Manual of Mental Disorders "DSM-5”, published in May 2013, approved the umbrella term ASD, with no subtypes. It divided the impairments into two domains: difficulties in social communication and social interaction; and restricted and repetitive behaviour, interests, or activities (American Psychiatric Association, 2013a). The diagnosis of ASD may include physical examinations which are designed to rule out any possible physical causes of difficulties, speech and language assessment, observation, and a history of the child's development and behaviour, focusing on features consistent with the DSM-5 or ICD-10 criteria (National Health Service, 2019; National Institute for Health and Care Excellence, 2017). Commonly used tools to aid diagnosis include the Autism Diagnostic Interview -Revised (ADI-R) and Autism Diagnostic Observation 
Schedule (ADOS-2) (National Institute for Health and Care Excellence, 2017). The ADI-R is a semi-structured interview that is conducted by a clinician along with a parent/carer who is familiar with the developmental history and the current behaviour of the child (Lord et al., 1994). The ADOS-2 has 5 modules which involve 40-60 minute protocols of activities that are based on playing, communication and social interactions (Lord et al., 2012).

Research shows that there is typically a delay of approximately 20 months to 5 years between parents' first noticing concerns about the development of their child and receiving a confirmed diagnosis of ASD (Crane et al., 2015; Howlin \& Asgharian, 1999; Nazneen, Matthews et al., 2015; Wiggins et al., 2006). This may result in delayed access to early intervention services, which are critical to ensure positive outcomes (Matson, 2008; McEachin et al., 1993; Rattazzi, 2014; Siegel, 2008; Smith et al., 2017). The long waiting times can also cause stress to individuals and families (Crane et al., 2015; Zwaigenbaum et al., 2016). Some of the reasons for the delays in diagnosis include a shortage of healthcare professionals (Thomas et al., 2007) and several specialist and treatment appointments are often needed to make a diagnosis. Multiple meetings with a variety of professionals in different locations can also be very stressful for individuals who might later be diagnosed with ASD. This is because people with ASD are very sensitive to changes in their situation and excessively reliant on routines (American Psychiatric Association, 2013a). Some families also have to travel long distances to access these services (Oberleitner et al., 2007) meaning both a cost to the family and also to the specialist healthcare teams who must travel to different areas. Recent research indicates that healthcare and educational systems work independently of each other, with little crossover of activity (Hurt et al., 2019). It could therefore be difficult to get multi-disciplinary teams together, especially when they span several different disciplines. Overall, there seems to be some evidence to indicate that parents of children with ASD and adults face difficulties in getting an ASD diagnosis. Therefore, more information is needed about how diagnostic services could be improved upon, to address the well-being of the child and improve the experiences of the parents and adults with ASD. 
Telehealth can provide help and support to people with specific health and well-being needs. Telehealth is identified as a mechanism that allows people to obtain professional help and services at a distance (Vismara et al., 2013). This may involve a Real-Time or Store-and-Forward methods. Real-Time interaction allows the patient to communicate in real time with a health care provider. Videoconferencing is viewed as one of the main forms of Real-Time communication for telehealth programs. Storeand-Forward interaction on the other hand, does not depend on the simultaneous presence of parties (e.g. healthcare provider and patient) (Maheu et al., 2001).

Telehealth may have advantages over traditional face to face approaches, including the fact that it could particularly improve access to healthcare services for individuals and families living in rural and underserved areas (Reese, Jamison et al., 2015). There is growing evidence to suggest that telehealth approaches could decrease costs for both providers and patients (e.g. travel time, transportation expenses, work absence) and increase coverage area to providers (Dunn, Choi, Almagro, Recla, \& Davis, 2000; Gros et al., 2013; Trott \& Blignault, 1998). Users of such programs can also interact directly with clinicians as well as with instructional content via video, email and apps systems (Brooke Ingersoll \& Wainer, 2013; Wainer \& Ingersoll, 2015). By offering families the opportunity to play a significant role in the development of their child, telehealth technology could expedite both the diagnostic process and early intervention services (Nazneen, Matthew et al., 2015; Wainer \& Ingersoll, 2015).

However, limited access to the necessary technologies, such as computers and mobile phones, could hamper the widespread use of telehealth (Alkhalifah \& Aldhalaan, 2018). In previous telehealth evaluations for children with ASD, good internet access was required (Vismara et al., 2013; Vismara et al., 2012; Wainer \& Ingersoll, 2015). This could be challenging for some communities, especially rural and underserved areas may be disadvantaged by a lack of mobile coverage (Iacono et al., 2016). Slow internet speed, poor quality and unreliable connectivity problems may result in frustration and lead to a general unwillingness to use telehealth approaches (Alkhalifah \& Aldhalaan, 2018). Some families were apparently worried that their children may change their behaviours, if they realised that they were being observed (Chedid, Dew, \& Veitch, 2013; Nazneen, Matthews et al., 2015). A further challenge to the use of telehealth is the apparent lack of skills and confidence when using technology amongst 
some individuals (Iacono et al., 2016). Service providers themselves might also be reluctant to engage with technology for several reasons. They might have limited experience in using remote communication systems, and could also be concerned about the impact this might have on their interactions with adults, children and their families (Chedid et al., 2013; Dunkley, Pattie, Wilson, \& McAllister, 2010).

The previous literature reported that telehealth is currently used by numerous clinical applications with positive results, such as tele-radiology (Al-Safadi, 2016), telecardiology (Hsieh et al., 2013), and monitoring the condition of home-based patients with diabetes and hypertension (Maheu et al., 2001) in addition to tele-mental health services (Hilty et al., 2013). Prior studies also showed that telehealth may be a promising way in which to provide a diagnosis in different conditions. For instance, when determining a diagnosis of dementia, it has been found that telehealth was as accurate as a face to face clinical examination with a high degree of satisfaction correlated with using telehealth (Shores et al., 2004). The research also demonstrates that using telemedicine for a congenital heart disease diagnosis is both accurate and safe (McCrossan et al., 2008). Furthermore, telehealth which used slit lamp images was suggested to be a reliable and accurate way in which to diagnose eye problems within accident and emergency departments (Bowman et al., 2003).

On account of such apparent benefits for these other clinical applications, telehealth was considered in the field of ASD (Aresti-Bartolome \& Garcia-Zapirain, 2014). The position of telehealth in the field of ASD is small but growing. Telehealth approaches have recently been explored as a way of supporting the delivery of a range of services for people with ASD and their families. Telehealth could be used to improve access to behavioural intervention services. Several studies have used telehealth to coach the parents of children with ASD how to conduct behavioural assessments such as functional analyses (FA) and functional communication training (FCT) via the use of videoconferencing. The parents and child could be located at a regional clinic, home or school (Lindgren et al., 2016; Machalicek et al., 2016; Simacek et al., 2017; Suess et al., 2014; Suess et al., 2016; Wacker, Lee, Dalmau, Kopelman, Lindgren, Kuhle, Pelzel, Dyson, et al., 2013; Wacker, Lee, Dalmau, Kopelman, Lindgren, Kuhle, Pelzel, \& Waldron, 2013). The findings suggested that parents can successfully conduct FA and FCT through telehealth when a behaviour analyst can offer consultation remotely. 
It has also been found to lead to reductions in the problem behaviour of children. For example, Wacker, Lee, Dalmau, Kopelman, Lindgren, Kuhle, Pelzel, Dyson, et al. (2013) found the average reduction in problem behaviour to be $93.5 \%$ when telehealth used. Similarly, Lindgren et al. (2016) revealed that problem behaviour decreased by an average of $90 \%$. The preliminary efficacy of telehealth-delivered parent mediated interventions has also been demonstrated. These are intended to increase parental knowledge and the use of ASD behavioural intervention strategies with the child in their daily life. Several studies suggested that use of a telehealth program, combining web-based instructional content with weekly video-conferencing coaching sessions may support parental learning and improve a child's social communication skills (Ingersoll \& Berger, 2015; Pickard et al., 2016; Vismara et al., 2013; Vismara et al., 2012; Wainer \& Ingersoll, 2015). Parents also indicated that such systems were effective, acceptable and useable.

Findings from these studies provide initial evidence for the feasibility, acceptability and effectiveness of telehealth technologies to serve as models for delivering parental training. Such training would support conducting the behavioural interventions and generally help parents better understand and use intervention practices in their daily interactions with children. Given this success, it is possible that telehealth may also be useful in improving the diagnostic pathway of ASD.

It would appear that so far, very limited research has been conducted in the literature regarding the use of telehealth in ASD. For example, a review by Boisvert et al. (2010) included only those studies that used telepractice directly with the individuals with ASD, but did not include studies that involved others such as parents of children with ASD. Other previous reviews (Michelle Boisvert \& Hall, 2014; Meadan \& Daczewitz, 2015) have focused on studies that centred around the use of telehealth and Internetbased interventions for training for parents or caregivers. However, to date, no previous literature review has specifically examined the use of telehealth in supporting ASD diagnostic assessment. Our review aims to fill this gap and builds upon previous work by providing a scoping review to examine which telehealth approaches have been used in the diagnosis and assessment of ASD in children and adults, whether they are feasible and acceptable, and how they compare with face to face diagnosis and assessment methods. 


\subsection{Method}

A scoping review was conducted to broadly find out what is known about using telehealth for ASD diagnostic assessment. There is a knowledge gap about the methods that used for ASD remote diagnosis, the feasibility, acceptability and accuracy of such approaches when compared with in-person methods. Contrary to a systematic review, which mainly focuses on identifying and appraising the quality of the evidence that is relevant to a particular question/questions (Munn et al., 2018), a scoping review investigates an area of literature more broadly, regardless of methodological quality, with the intention of examining the extent, nature, and range of research activity (Arksey \& O'Malley, 2005; Munn et al., 2018). Therefore, a scoping review was considered to be preferable to a systematic review as a means of achieving the study aim. The PRISMA extension for scoping reviews (PRISMA-ScR) checklist was used to ascertain the key items required to report for a scoping review (see Appendix 2) (Tricco et al., 2018).

\subsubsection{Search strategy}

The following electronic bibliographic databases were used: MEDLINE, CINAHL Plus with Full text, Business Sources Complete, Web of Science, Scopus and PsycINFO and trial and systematic review databases including Cochrane Library, Health Technology Assessment, Database of Abstracts and Reviews of Effectiveness and NHS Economic Evaluation. Online sources searched included guidelines, government health sites, and ASD related associations. Searches were limited to the English Language from January 2000 to December 2019 inclusive. The rationale for not including studies prior to 2000 is that telehealth technology was not introduced to the ASD field until 2004 (Aresti-Bartolome \& Garcia-Zapirain, 2014). However, the literature search was covered the period from January 2000 to ensure that there were no studies that could be consistent with our inclusion criteria. The terms for autism were combined with terms for telehealth to identify eligible studies (see Box 1 an example of an actual full search in MEDLINE database). 


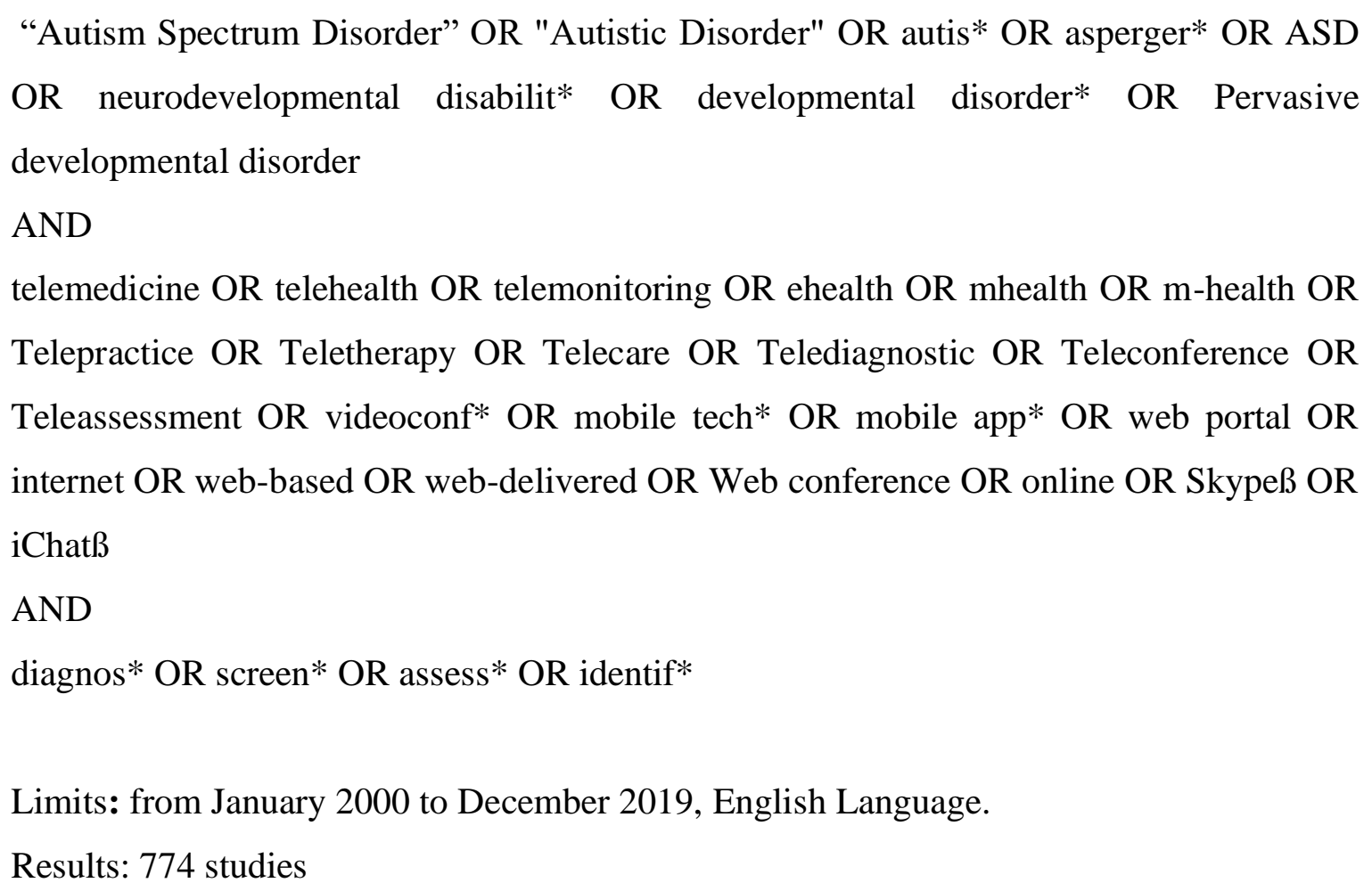

\subsubsection{Inclusion and exclusion criteria}

The inclusion criteria specified that studies should involve telehealth technology and should report outcomes (not merely describe a service). We define telehealth technology as being any technology that provides ASD diagnostic assessment services at a distance. With regards to the outcomes, as this review is comprehensive, we are considering all of the outcomes reported when telehealth has been used in the diagnosis and assessment of ASD in children and adults, e.g. the feasibility and acceptability of telehealth approaches in the diagnosis and assessment of ASD, as well as the accuracy of remote diagnosis when compared with in-person methods. As this is a developing field, we included all study designs so as to fully understand the current state of the literature. Only primary studies were included. The study population included anyone going through an ASD diagnosis process with any type of healthcare practitioner. Any study that considered only technical issues, editorials, opinions or those concerned with medical education, were excluded. In the case of studies involving children include (younger than 18 years old), information was usually 
reported by parents/carers, and in the case of studies involving adults (18 years old and older), the information could either be given by the adults themselves or their carers.

\subsubsection{Data extraction and synthesis}

Articles, abstracts or titles identified from the search strategy were reviewed by MA to determine if they met the inclusion criteria. In cases where the eligibility of the study was unclear, it was reviewed by a second reviewer (SB or JC). The full texts were retrieved and reviewed in full by two independent researchers (MA $+\mathrm{SB} / \mathrm{JC}$ ). There was no disagreement in terms of agreement and a third person did not need to adjudicate. Author, year of publication, country, participant characteristics, study design, technology utilised, services delivered, and outcomes were extracted for each paper using a standard form in Excel.

\subsubsection{Quality assessment}

Independent assessment of the methodological quality was conducted by two authors (MA, SB or JC) for the included studies. In the event of any differences, consensus was reached by discussion or through consulting (SB or JC). The National Heart, Lung, and Blood Institute (NHLBI) Quality Assessment for Observational Cohort and Cross-Sectional Studies tool (NHLBI, n.d.) was used for five studies (Nazneen, Rozga et al., 2015; Parmanto, Pulantara, Schutte, Saptono, \& McCue, 2013; Reese, Braun et al., 2015; Schutte et al., 2015; Stainbrook et al., 2018). Each item was evaluated by using "yes", "no", "not applicable", "cannot determine", or "not reported". This was used to guide the overall rating for the quality of each study as "good", "fair", or "poor". The Diagnostic research Critical Appraisal Skills Programme (CASP) tool (Critical Appraisal Skills Programme, 2018) was also used for four studies (Juarez et al., 2018; Reese, Jamison et al., 2015; Reese et al., 2013; Smith et al., 2017) and the Qualitative research Critical Appraisal Skills Programme (CASP) tool (Critical Appraisal Skills Programme, 2018) for one study (Nazneen, Matthews et al., 2015). Each item was assessed by using "yes", "no", or "cannot tell". As there are no quality scores in the CASP tool, it has decided to use the same quality scores of the NHLBI quality assessment tools ("good", "fair”, or "poor”) (NHLBI, n.d.) for each study. 


\subsubsection{Publication and roles of the authors:}

This scoping review has been published in PLOS ONE Journal as shown below:

"Alfuraydan, M., Croxall, J., Hurt, L., Kerr, M., \& Brophy, S. (2020). Use of Telehealth for Facilitating the Diagnostic Assessment of Autism Spectrum Disorder (ASD): A Scoping Review. PloS one, 15(7), 1-16. “

The researcher Manahil Alfuraydan was the first author who made the most contribution to this scoping review. The roles of the researcher include the conceptualisation and designing of the study and writing the original draft, including reviewing and editing. Moreover, conducting the methods, including performing the search strategy, identifying the eligible studies, screening and reviewing the titles, abstracts and articles that identified from the search strategy, in order to determine if they met the inclusion criteria. In cases where the eligibility of a study was unclear, it was reviewed by a second reviewer ( $\mathrm{SB}$ or $\mathrm{JC}$ ), and an assessment of the methodological quality of all 10 included studies was conducted. The authors Dr Jodie Croxall and Prof. Sinead Brophy provided supervision and offered some feedback on the drafts of the scoping review. They also have contribution with the first author in the parts of the screening process and quality assessment, in order to make independent assessment and comparison. Regarding the data extraction and synthesis, the full texts were retrieved and reviewed in full by two independent authors (MA, SB/JC). There was no disagreement during this process and it was not necessary for a third person to adjudicate. Furthermore, independent assessment of the methodological quality of the included studies was conducted by two authors (MA, SB/JC). So, the first author worked on 10 articles and Prof. Sinead Brophy worked on 5 articles and Dr Jodie Croxall worked on the other 5 articles. In the event of there being any differences, consensus was reached either by discussion or through consulting (SB or JC). The other authors Dr Lisa Hurt and Prof. Mike Kerr provided feedback and comments on the first draft of the scoping review. 


\subsection{Results}

\subsubsection{Study characteristics}

A total of 3698 articles were identified using the search strategy, of which 124 were selected for abstract screening based on title screening. Of these, 37 were selected for full text review and 10 papers were selected for inclusion in the review and data extraction (see Figure 3-1). The studies that were rejected had mainly examined treatment of ASD using telehealth rather than diagnosis. In addition, studies that were reviews, clinical letters, or including a non ASD population or not using telehealth technologies were rejected. All 10 studies were based in the USA and published between 2013 and 2018 (see Table 3-1). There were 5 independent research teams as 3 papers were by Reese et al. (Reese, Braun et al., 2015; Reese, Jamison et al., 2015; Reese et al., 2013), 2 were by Nazeen et al. (Nazneen, Matthews et al., 2015; Nazneen, Rozga et al., 2015), 2 were with Parmanto and team (Parmanto et al., 2013; Schutte et al., 2015), 2 were with Juarez and team (Juarez et al., 2018; Stainbrook et al., 2018) and one study was by Smith et al. (Smith et al., 2017). A total of 997 people participated across the 10 papers and of these 629 were parents, 291 were children (with ASD) 33 were adults (with ASD) and 44 were clinicians. 


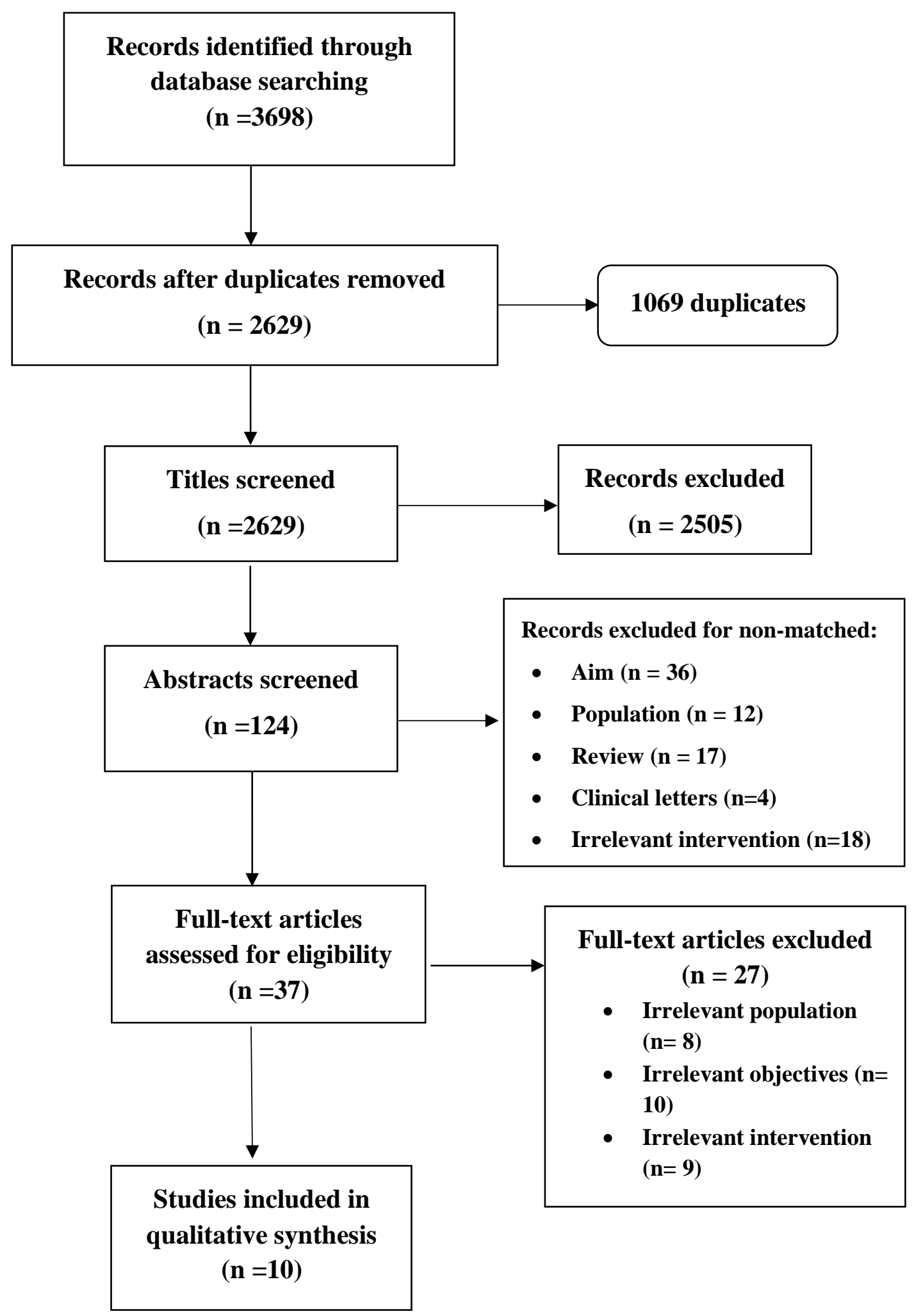

Figure 3-1: Flowchart of study selection process 
Table 3-1: Summary of study characteristics

\begin{tabular}{|c|c|c|c|c|c|c|c|}
\hline $\begin{array}{c}\text { Author, } \\
\text { year }\end{array}$ & Country & $\begin{array}{l}\text { Participants } \\
\text { Characteristics }\end{array}$ & Study design & Categorisation & $\begin{array}{c}\text { Technology } \\
\text { utilized }\end{array}$ & $\begin{array}{c}\text { Service } \\
\text { delivered }\end{array}$ & Outcomes \\
\hline $\begin{array}{l}\text { Nazneen, } \\
\text { Matthews } \\
\text { et al., 2015) }\end{array}$ & USA & $\begin{array}{l}43 \text { parents, } 51 \\
\text { target children } \\
\text { and their } \\
\text { siblings, and } 10 \\
\text { diagnosticians. } \\
\text { Children were } \\
\text { between the } \\
\text { ages of } 18 \\
\text { months and } 6 \\
\text { years, } 11 \\
\text { months. }\end{array}$ & $\begin{array}{l}\text { Qualitative study } \\
\text { Interview }\end{array}$ & Qualitative & NODA & $\begin{array}{l}\text { Remote ASD } \\
\text { diagnosis }\end{array}$ & $\begin{array}{l}\text { Parents and } \\
\text { diagnosticians } \\
\text { found that NODA } \\
\text { is easy to use and } \\
\text { effective approach } \\
\text { to address the } \\
\text { obstacles to } \\
\text { autism diagnosis. } \\
\text { They reported } \\
\text { possible barriers } \\
\text { such as privacy } \\
\text { concerns and } \\
\text { reactivity of } \\
\text { behaviour. }\end{array}$ \\
\hline $\begin{array}{l}\text { (Nazneen, } \\
\text { Rozga et } \\
\text { al., 2015) }\end{array}$ & USA & $\begin{array}{l}\text { Stage 1: one- } \\
\text { on-one } \\
\text { interview: } 7 \\
\text { parents of } \\
\text { children with } \\
\text { autism and } 11 \\
\text { clinicians. } \\
\text { Stage 2: } \\
\text { iterative } \\
\text { development: } 8 \\
\text { families, } 18 \\
\text { children and } \\
\text { their siblings }\end{array}$ & $\begin{array}{l}\text { Pilot study } \\
\text { Interview, } \\
\text { development, and } \\
\text { evaluation. }\end{array}$ & Observational & NODA & $\begin{array}{l}\text { Remote ASD } \\
\text { diagnosis }\end{array}$ & $\begin{array}{l}\text { Parents can easily } \\
\text { record videos of } \\
\text { their children } \\
\text { behaviours. The } \\
\text { clinical validity } \\
\text { ratings of the } \\
\text { collected videos } \\
\text { were } 96 \% \text {. For } 4 \\
\text { out of } 5 \text { children } \\
\text { were remotely } \\
\text { diagnosed by both } \\
\text { diagnosticians that } \\
\text { matched with the }\end{array}$ \\
\hline
\end{tabular}




\begin{tabular}{|c|c|c|c|c|c|c|c|}
\hline & & $\begin{array}{l}\text { Stage3: in-field } \\
\text { evaluation: } 4 \\
\text { families with at } \\
\text { least } 1 \text { child } \\
\text { with previous } \\
\text { ASD diagnosis } \\
\text { and } 1 \text { family } \\
\text { with a typically } \\
\text { developing } \\
\text { child and } 2 \\
\text { diagnosticians. } \\
\text { Children were } \\
\text { between } 2 \text { and } \\
6 \text { years of age. }\end{array}$ & & & & & $\begin{array}{l}\text { child's actual } \\
\text { diagnosis. }\end{array}$ \\
\hline $\begin{array}{l}\text { (Smith et } \\
\text { al., 2017) }\end{array}$ & USA & $\begin{array}{l}40 \text { families } \\
\text { seeking an ASD } \\
\text { assessment for } \\
\text { their children } \\
\text { and } 11 \text { families } \\
\text { of typically } \\
\text { developing } \\
\text { children. } \\
\text { Children were } \\
\text { between the } \\
\text { ages of } 18 \\
\text { months and } 6 \\
\text { years } 11 \\
\text { months }\end{array}$ & $\begin{array}{l}\text { Diagnostic } \\
\text { accuracy study (In- } \\
\text { person } \\
\text { assessment/NODA) }\end{array}$ & Diagnostic & $\begin{array}{c}\text { NODA } \\
.\end{array}$ & $\begin{array}{l}\text { ASD remote } \\
\text { diagnosis }\end{array}$ & $\begin{array}{l}\text { There was } \\
\text { substantial } \\
\text { agreement } \\
\text { between NODA } \\
\text { and in-person } \\
\text { assessment } \\
\text { (IPA) }\end{array}$ \\
\hline $\begin{array}{l}\text { (Reese et } \\
\text { al., 2013) }\end{array}$ & USA & $\begin{array}{l}11 \text { children } \\
\text { with autism and } \\
10 \text { with an } \\
\text { existing }\end{array}$ & $\begin{array}{l}\text { Pilot study } \\
\text { Diagnostic } \\
\text { accuracy study }\end{array}$ & Diagnostic & $\begin{array}{l}\text { Video- } \\
\text { conferencing }\end{array}$ & $\begin{array}{l}\text { Conducting } \\
\text { ASD } \\
\text { assessment }\end{array}$ & $\begin{array}{l}\text { No significant } \\
\text { difference in the } \\
\text { diagnostic }\end{array}$ \\
\hline
\end{tabular}




\begin{tabular}{|c|c|c|c|c|c|c|c|}
\hline & & $\begin{array}{l}\text { diagnosis of } \\
\text { developmental } \\
\text { delay }(n=21) \text {. } \\
\text { Ages: 3-5 years } \\
\text { old. }\end{array}$ & & & & $\begin{array}{l}\text { (ADOS-2 } \\
\text { and ADI-R) } \\
\text { Diagnosis }\end{array}$ & $\begin{array}{l}\text { accuracy, ratings } \\
\text { for ADI-R, } \\
\text { ADOS } \\
\text { observations, } \\
\text { and parent } \\
\text { satisfaction } \\
\text { between IVC } \\
\text { and InP } \\
\text { conditions. }\end{array}$ \\
\hline $\begin{array}{l}\text { (Reese, } \\
\text { Jamison et } \\
\text { al., 2015) }\end{array}$ & USA & $\begin{array}{l}17 \text { families of } \\
\text { young children } \\
\text { requesting an } \\
\text { evaluation for } \\
\text { ASD. } \\
\text { Ages:2.5-6 } \\
\text { years old. }\end{array}$ & $\begin{array}{l}\text { Diagnostic } \\
\text { accuracy study }\end{array}$ & Diagnostic & $\begin{array}{l}\text { Video- } \\
\text { conferencing }\end{array}$ & $\begin{array}{l}\text { Conducting } \\
\text { ASD } \\
\text { assessment } \\
\text { (ADOS-2 } \\
\text { and ADI-R) } \\
\text { Diagnosis }\end{array}$ & $\begin{array}{l}\text { Results revealed } \\
\text { excellent } \\
\text { interrater } \\
\text { agreement on } \\
\text { diagnoses } \\
\text { between } \\
\text { clinicians in the } \\
\text { VC setting and } \\
\text { interdisciplinary } \\
\text { assessment team. }\end{array}$ \\
\hline $\begin{array}{l}\text { (Reese, } \\
\text { Braun et } \\
\text { al., 2015) }\end{array}$ & USA & 370 families & Retrospective study & Observational & ISUT & $\begin{array}{l}\text { Examining } \\
\text { telehealth's } \\
\text { impact on } \\
\text { families' } \\
\text { access to } \\
\text { diagnostic } \\
\text { services } \\
\end{array}$ & $\begin{array}{l}\text { ISUT provided } \\
\text { families increased } \\
\text { access to } \\
\text { diagnostic } \\
\text { services. }\end{array}$ \\
\hline $\begin{array}{r}\text { (Parmanto } \\
\text { et al., 2013) }\end{array}$ & USA & $\begin{array}{l}5 \text { clinicians } \\
10 \text { adults with } \\
\text { disability }\end{array}$ & Case study & Observational & Videoconferencing & $\begin{array}{l}\text { Remote } \\
\text { assessment of } \\
\text { adults with }\end{array}$ & $\begin{array}{l}\text { The patients were } \\
\text { satisfied with the } \\
\text { developed } \\
\text { telehealth system }\end{array}$ \\
\hline
\end{tabular}




\begin{tabular}{|c|c|c|c|c|c|c|c|}
\hline & & $\begin{array}{l}\text { Age: } 17 \text { years } \\
\text { old }\end{array}$ & & & & $\begin{array}{l}\text { ASD (ADOS } \\
\text { Module 4) }\end{array}$ & $\begin{array}{l}\text { and provided } \\
\text { feedback that it } \\
\text { was easy to use }\end{array}$ \\
\hline $\begin{array}{l}\text { (Schutte et } \\
\text { al., 2015) }\end{array}$ & USA & $\begin{array}{l}23 \text { adults with } \\
\text { an ASD } \\
\text { diagnosis. } \\
\text { Ages: } 22 \text { years } \\
\text { old }\end{array}$ & $\begin{array}{l}\text { Within-subject } \\
\text { crossover study. } \\
\text { Questionnaire. }\end{array}$ & Observational & $\begin{array}{l}\text { Versatile and } \\
\text { Integrated System } \\
\text { for Tele- } \\
\text { rehabilitation } \\
\text { (VISYTER). It } \\
\text { consists of } \\
\text { videoconferencing } \\
\text { and web portal. }\end{array}$ & $\begin{array}{l}\text { Remote } \\
\text { assessment of } \\
\text { adults with } \\
\text { ASD (ADOS } \\
\text { Module 4) }\end{array}$ & $\begin{array}{l}\text { Participant } \\
\text { satisfaction } \\
\text { was high. It } \\
\text { demonstrated that } \\
\text { an adult autism } \\
\text { assessment can be } \\
\text { administered } \\
\text { distantly with high } \\
\text { levels of } \\
\text { reliability. }\end{array}$ \\
\hline \multirow[t]{2}{*}{$\begin{array}{l}\text { (Juarez et } \\
\text { al., 2018) }\end{array}$} & \multirow[t]{2}{*}{ USA } & $\begin{array}{l}\text { Study1: } 20 \\
\text { children and } \\
\text { their caregivers } \\
\text { (16 boys, } 4 \\
\text { girls) } \\
\text { Ages: between } \\
20 \text { and } 34 \\
\text { months. }\end{array}$ & \multirow[t]{2}{*}{$\begin{array}{l}\text { Study1: Diagnostic } \\
\text { accuracy study } \\
\text { Study2: Feasibility } \\
\text { study }\end{array}$} & \multirow[t]{2}{*}{$\begin{array}{c}\text { Diagnostic/ } \\
\text { Observational }\end{array}$} & \multirow[t]{2}{*}{$\begin{array}{l}\text { Video- } \\
\text { conferencing }\end{array}$} & \multirow[t]{2}{*}{$\begin{array}{l}\text { ASD remote } \\
\text { diagnosis }\end{array}$} & $\begin{array}{l}\text { Study1: all of the } \\
\text { children }(\mathrm{n}=15) \\
\text { who received } \\
\text { ASD diagnosis via } \\
\text { telehealth were } \\
\text { confirmed by face } \\
\text { to face } \\
\text { assessment. }\end{array}$ \\
\hline & & $\begin{array}{l}\text { Study2: } 45 \\
\text { children and } \\
\text { their caregivers } \\
(35 \text { boys, } 10 \\
\text { girls) } \\
\text { Ages: between } \\
19 \text { and } 32 \\
\text { months. }\end{array}$ & & & & & $\begin{array}{l}\text { Study2: clinicians } \\
\text { were satisfied with } \\
\text { the telemedicine } \\
\text { in } 80 \% \text { of cases ( } \mathrm{n} \\
=36 \text { ) but would } \\
\text { rather to assess a } \\
\text { child in person } \\
24 \% \text { of the time ( } \mathrm{n} \\
=11 \text { ). }\end{array}$ \\
\hline
\end{tabular}




\begin{tabular}{|c|c|c|c|c|c|c|}
\hline $\begin{array}{l}\text { (Stainbrook } \\
\text { et al., 2018) }\end{array}$ & 63 families & Retrospective study & Observational & $\begin{array}{l}\text { Video- } \\
\text { conferencing }\end{array}$ & $\begin{array}{l}\text { Measuring } \\
\text { Telediagnosis } \\
\text { impact on } \\
\text { referrals }\end{array}$ & $\begin{array}{l}\text { Telemedicine } \\
\text { diagnostic } \\
\text { consultation may } \\
\text { positively impact } \\
\text { referrals for } \\
\text { diagnostic } \\
\text { evaluation }\end{array}$ \\
\hline
\end{tabular}

Note. $\mathrm{ASD}=$ Autism Spectrum Disorder, USA= United States of America, NODA= Naturalistic Observation Diagnostic Assessment, ADOS =Autism Diagnostic Observation Schedule, ADOS-2= Autism Diagnostic Observation Schedule, Second Edition, ADI-R= Autism Diagnostic Interview - Revised, IVC=Interactive

Videoconferencing, ISUT= Integrated System Using Telemedicine, VISYTER= Versatile and Integrated System for Tele-rehabilitation. 


\subsubsection{Quality assessment}

Quality assessment tools from the NHLBI and CASP were used to assess the methodological quality of the included studies. The overall quality was rated as good, fair, or poor. Eight studies were rated as being of "fair" quality (Juarez et al., 2018; Nazneen, Rozga et al., 2015; Reese, Braun et al., 2015; Reese, Jamison et al., 2015; Reese et al., 2013; Schutte et al., 2015; Smith et al., 2017; Stainbrook et al., 2018) whereas, two studies were rated as being poor-quality assessment (Nazneen, Matthews et al., 2015; Parmanto et al., 2013). In general, most of the studies had a small sample size. There were also issues with a lack of clarity around research questions and objectives in some studies; insufficient details were provided about sampling, recruitment strategies and inclusion and exclusion criteria; there was a lack of clarity in the rigor of the data analysis and reporting of ethics (see Table 3-2, Table 3-3, Table 3-4). 
Table 3-2: Results of quality assessment tool (from CASP) for diagnostic studies.

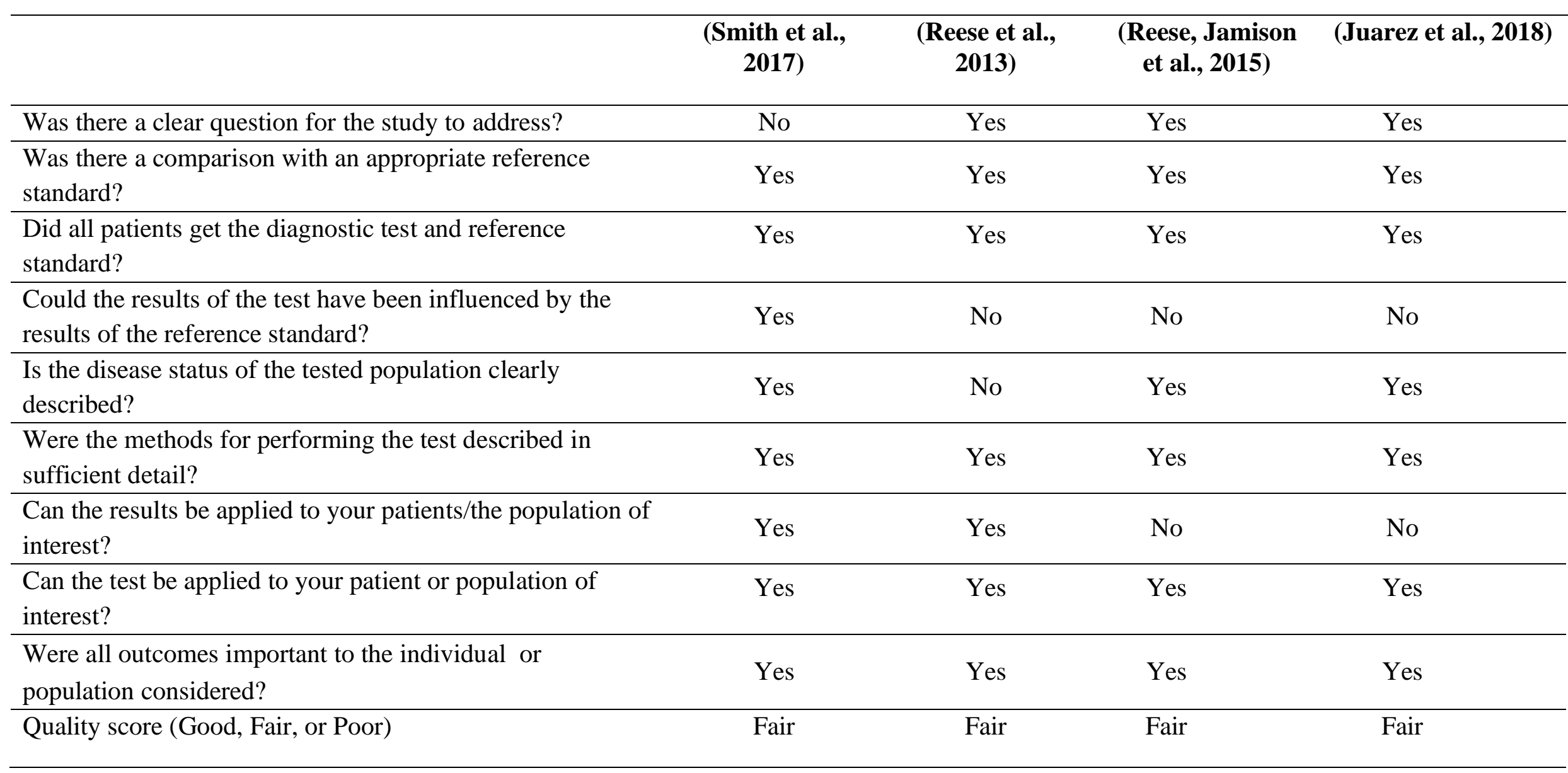

Available at: https://casp-uk.net/casp-tools-checklists/ 
Table 3-3: Results of quality assessment tool (from the NHLBI) for observational cohort and cross-sectional studies

\begin{tabular}{|c|c|c|c|c|c|}
\hline & $\begin{array}{l}\text { (Reese, } \\
\text { Braun et } \\
\text { al., 2015) }\end{array}$ & $\begin{array}{l}\text { (Parmanto } \\
\text { et al., 2013) }\end{array}$ & $\begin{array}{l}\text { (Stainbrook et } \\
\text { al., 2018) }\end{array}$ & $\begin{array}{l}\text { (Schutte et } \\
\text { al., 2015) }\end{array}$ & $\begin{array}{l}\text { (Nazneen, } \\
\text { Rozga et al., } \\
\text { 2015) }\end{array}$ \\
\hline $\begin{array}{l}\text { Was the research question or objective in this paper clearly } \\
\text { stated? }\end{array}$ & Yes & No & Yes & Yes & Yes \\
\hline \multicolumn{6}{|l|}{ Was the study population clearly specified and defined? } \\
\hline & Yes & Yes & $\mathrm{CD}$ & Yes & No \\
\hline Was the participation rate of eligible persons at least $50 \% ?$ & No & NA & $\mathrm{CD}$ & Yes & NA \\
\hline $\begin{array}{l}\text { Were all the subjects selected or recruited from the same or } \\
\text { similar populations (including the same time period)? Were } \\
\text { inclusion and exclusion criteria for being in the study } \\
\text { prespecified and applied uniformly to all participants? }\end{array}$ & No & NR & Yes & Yes & NR \\
\hline $\begin{array}{l}\text { Was a sample size justification, power description, or variance } \\
\text { and effect estimates provided? }\end{array}$ & Yes & No & Yes & NA & No \\
\hline $\begin{array}{l}\text { For the analyses in this paper, were the exposure(s) of interest } \\
\text { measured prior to the outcome(s) being measured? }\end{array}$ & Yes & NA & Yes & Yes & NA \\
\hline $\begin{array}{l}\text { Was the timeframe sufficient so that one could reasonably } \\
\text { expect to see an association between exposure and outcome if it } \\
\text { existed? }\end{array}$ & Yes & $\mathrm{CD}$ & Yes & Yes & $\mathrm{CD}$ \\
\hline
\end{tabular}


For exposures that can vary in amount or level, did the study examine different levels of the exposure as related to the outcome (e.g., categories of exposure, or exposure measured as

NA

No

NA

NA

NA

continuous variable)?

Were the exposure measures (independent variables) clearly defined, valid, reliable, and implemented consistently across all study participants?

Was the exposure(s) assessed more than once over time?

?

Were the outcome measures (dependent variables) clearly
defined, valid, reliable, and implemented consistently across all

study participants?

Were the outcome assessors blinded to the exposure status of participants?

Was loss to follow-up after baseline $20 \%$ or less?

Yes

Yes

Yes

Yes

Yes

No

NA

NA

No

NA

Were key potential confounding variables measured and adjusted statistically for their impact on the relationship between exposure(s) and outcome(s)?

Quality score (Good, Fair, or Poor)

Fair

Poor

Yes

Yes

Yes

Yes

No CD

$\mathrm{CD}$

CD

No

Yes

NA

NA

NA

NA

Available at: https://www.nhlbi.nih.gov/health-topics/study-quality-assessment-tools 
Table 3-4: Results of quality assessment tool (from CASP) for qualitative study

(Nazneen, Matthews et al., 2015)

\begin{tabular}{ll}
\hline Was there a clear statement of the aims of the research? & Yes \\
\hline Is a qualitative methodology appropriate? & Yes \\
\hline $\begin{array}{l}\text { Was the research design appropriate to address the aims of the } \\
\text { research? }\end{array}$ & Can't tell \\
\hline $\begin{array}{l}\text { Was the recruitment strategy appropriate to the aims of the } \\
\text { research? }\end{array}$ & No \\
\hline $\begin{array}{l}\text { Was the data collected in a way that addressed the research } \\
\text { issue? }\end{array}$ & No \\
\hline $\begin{array}{l}\text { Has the relationship between researcher and participants been } \\
\text { adequately considered? }\end{array}$ & No \\
\hline Have ethical issues been taken into consideration? & No \\
\hline Was the data analysis sufficiently rigorous? & Can't tell \\
\hline Is there a clear statement of findings? & Poor \\
\hline Quality score (Good, Fair, or Poor) & \\
\hline
\end{tabular}

Available at: https://www.nhlbi.nih.gov/health-topics/study-quality-assessment-tools $\mathrm{CD}$, cannot determine. 


\subsubsection{Telehealth technologies}

Regarding the assessed technologies, 7 papers used the Real-Time method with 5 of these (Parmanto et al., 2013; Reese, Braun et al., 2015; Reese, Jamison et al., 2015; Reese et al., 2013; Schutte et al., 2015) using Videoconferencing and with 2 of them (Juarez et al. 2018; Stainbrook et al., 2018) using the Versatile and Integrated System for Tele-rehabilitation (VISYTER) system. A further 3 papers (Nazneen, Matthews et al., 2015; Nazneen, Rozga et al., 2015; Smith et al., 2017) used the Store-and-Forward method, particularly the Naturalistic Observation Diagnostic Assessment (NODA) system.

\subsubsection{Outcomes}

The outcomes are structured and reported according to the telehealth methods (RealTime/ Store-and-Forward) as each study had different results.

\subsubsection{Real-Time method}

Videoconferencing. Videoconferencing software enables the families, medical teams and educational teams to meet and discuss care in real time from multiple locations. Three of the studies were by Reese et al. (Reese, Braun et al., 2015; Reese, Jamison et al., 2015; Reese et al., 2013) and this series of studies made a connection via videoconferencing between the specialist hospital and the local health centre or school. Similarly, two studies (Parmanto et al., 2013; Schutte et al., 2015) used videoconferencing. The three studies by Reese et al. (Reese, Braun et al., 2015; Reese, Jamison et al., 2015; Reese et al., 2013) used simple videoconferencing to examine (1) the ability of the clinician to assess autism using videoconferencing, (2) the feasibility of clinicians being able to coach the patients using videoconferencing so they can correctly complete the assessment activities with their child and (3) the satisfaction of the families with the telemedicine assessment, as compared to standard face to face assessment. In terms of diagnostic accuracy, these studies found that the diagnosis assessment agreed with the evaluation team diagnosis; $82 \%(n=28 / 34)$ of the time (for the face to face assessment) and $86 \%(\mathrm{n}=24 / 28)$ (for the videoconference assessment) (Reese, Braun et al., 2015; Reese et al., 2013). There was no significant difference in 
the ratings for the ADI-R and ADOS observations between those conducted face to face and those conducted online (Reese et al., 2013). There was also excellent agreement between the clinicians across the diagnostic DSM-5 ASD criteria both in person and when using the videoconferencing approaches (Reese, Jamison et al., 2015). Percent agreement scores equal to or $>80 \%$ have been considered "excellent" agreement, with scores between 70 and 80\% "adequate" and scores under $70 \%$ considered inadequate. Therefore, these results indicate that there were no significant differences between the face to face and videoconferencing conditions.

The final study by Reese, Braun et al. (2015) found that access was improved and that there was an increase in the diagnostic services for families living in rural and underserved areas. There were 405 families who went through the standard face to face assessment and 370 who went through the telemedicine assessment. Only $16 \%$ returned the satisfaction survey. The children involved in the telemedicine method were from a wider area (74 counties compared to 35 involved in the face to face standard method), were diagnosed 3 years earlier with this method also identifying older children who had not been previously diagnosed. The travel costs for those who used the telemedicine system would have been substantially more if they had needed to travel for each visit ( $\$ 100$ average cost if the videoconferencing families had needed to travel versus $\$ 35$ average cost for face to face family). The parents were equally satisfied with both the videoconferencing and the face to face approaches.

Two studies by Juarez et al. (2018) and Stainbrook et al. (2018) examined the accuracy of diagnosis when using telehealth (videoconferencing) compared to the in-person assessment in 20 cases and feedback from both the families and clinicians on the acceptability of telemedicine (Juarez et al., 2018). The impact that telehealth had on the tertiary care referrals was also examined (Stainbrook et al., 2018). In terms of the accuracy of the diagnosis, using remote telemedicine procedures, the clinicians identified $75 \%$ of cases $(n=15 / 20)$ as having ASD. The clinicians rated themselves as "certain" or "very certain" about the classification for $75 \%$ of cases. Nineteen out of 20 children were diagnosed with ASD by the in-person clinician. A face-to-face assessment confirmed the diagnosis of all of the children $(100 \%, \mathrm{n}=15)$ who received the ASD diagnosis via telehealth. However, $20 \%$ of cases $(n=4)$ diagnosed by an in- 
person assessment were not diagnosed remotely. In a study of the feasibility and acceptability of obtaining an ASD diagnosis through telehealth ( $\mathrm{n}=45$ children), clinicians reported being 'certain' or 'very certain' of their assessments in $86.67 \%$ of cases $(n=39)$. The clinicians were 'satisfied' or 'very satisfied' with the telemedicine in $80 \%$ of cases $(n=36)$ but they would rather assess a child using an in-person assessment $24 \%$ of the time $(n=11)$, mainly for those children who were then referred for a full evaluation $(n=6)$. This was because of either some psychosocial factors including trauma history and complicated diagnostic profiles or technical issues with the telehealth platform ( $23.8 \%$ audio, $4.8 \%$ video quality concerns). However, $98 \%$ of caregivers were very satisfied with the telemedicine experience. The telehealth assessment saved an average of 3.92 hours anticipated travel time when compared to the families attending the hospital-based ASD clinic.

The study examining the impact of telehealth (videoconferencing) on tertiary care referrals (Stainbrook et al., 2018) found that the telehealth diagnostic consultation could positively impact referrals for the diagnostic assessment. Telehealth accelerated access to the diagnostic assessment when compared to waiting at the tertiary centre. For example, in 2017, the system referred 323 children for evaluation with a 15-month waiting time. In contrast, families waited 11 weeks for a telemedicine visit and kept more appointments, as they were able to schedule and attend their own appointments. Most families (56 out of 63) preferred the remote method rather than travelling to the tertiary centre. The referrals to the centre for a complete evaluation generally either involved those children with different presentations or was down to family preference.

Versatile and Integrated System for Tele-rehabilitation (VISYTER). Two studies (Parmanto et al., 2013; Schutte et al., 2015) used VISYTER, which incorporates videoconferencing, layout control, image and video presentations, an electronic scoring system and session recording/archiving. This was developed to support the diagnosis of adolescents and adults with possible ASD. It is a piece of "off the shelf" hardware with its own secure server system. There is one desk at the clinician's site and another at the local clinic (so the patient needs to attend the clinic in order to use the system). Two cameras are on the patient's side to capture their eye contact and hand movements. The clinician can zoom and control the camera and take snap shots. These two studies (Parmanto et al., 2013; Schutte et al., 2015) examined the use of the 
VISYTER system for the assessment of adults. The participants were between the ages of 19 to 30; 26 out of 46 met the inclusion/exclusion criteria. Three dropped out because they were absent for the second ADOS $(n=23)$. Of these, 14 used the remote method after using face to face method (Schutte et al., 2015). The majority had no preference and found both methods to be equal, 5 preferred face to face and 2 preferred remote. In this study, 17(74\%) of 23 individuals were comfortable using the remote system, $18(78 \%)$ of 23 individuals felt that the online system correctly represented their typical behaviours and interactions with others. The results supported the use of telehealth to conduct ADOS remotely. The majority of observations were comparable. However, there were differences in the "Empathy/Comments on others' Emotions", "Hand and Finger and other Complex Mannerisms" and "Asks for Information" which may be harder to assess remotely. In the team's other study (Parmanto et al., 2013), they developed Telehealth Usability Questionnaire by combining questions from three questionnaires relevant to telehealth systems: the Technology Acceptance Model's Perceived Usefulness/Ease of Use, the Computer System Usability Questionnaire, and the Telemedicine Satisfaction Questionnaire. A 7-point Likert scale has been used with 1 representing disagreement and 7 representing agreement. They found that the patients $(n=10)$ were satisfied with the remote system (average score of 6.5 out of 7) and that it scored high for learnability (6.14 out of 7), interface quality (6.5), interaction quality (6.3), that they were very comfortable with the system (6.5) and that they would use it again (6.17). However, the acceptability of telehealth as a substitute for face to face was lower (5.86 out of 7). 


\subsubsection{Store-and-Forward method}

Naturalistic Observation Diagnostic Assessment (NODA). Three studies (2 by the same author (Nazneen) (Nazneen, Matthews et al., 2015; Nazneen, Rozga et al., 2015; Smith et al., 2017) used the NODA system which includes NODA SmartCapture and NODA connect (Nazneen, Matthews et al., 2015). NODA SmartCapture enables the parents to upload short videos from their smart phones of their child's behaviour in specific situations (e.g. family meal, play time with others, playtime alone and a situation of parental concerns) and remotely share them with a clinician. The first three situations offered opportunities for the child to show play-based behaviours and socialcommunication skills. There are instructions for filming each separate situation, containing recording instructions and a sample video to suggest to the parents how the environment should be setup. The medical professionals can also send additional recording instructions to the parents, with it all being uploaded to a webportal. The developmental history of the child is included in the webportal as well. NODA Connect has a list of tags that allows the clinician to determine the presence or absence of behaviours (e.g. no eye contact). These tags are auto-mapped to the (DSM-5) diagnostic criteria. The assessment can be shared with other clinicians and the diagnostic report can be shared with the parents.

The two studies by Nazneen (Nazneen, Matthews et al., 2015; Nazneen, Rozga et al., 2015) both evaluate the system for development. They involved interviewing both clinicians and families and also an in-field evaluation, which aimed to describe the experience of the clinicians and parents as they use NODA. The NODA development study (Nazneen, Rozga et al., 2015) found that parents reported that it was easy to use, giving it a score of 4 on a 5-point scale. The parents were able to follow the instructions and collected videos of the correct length, with clinicians stating that $96 \%$ of videos recorded by the parents were clinically useful. In total, 10 out of 11 of the assessments were given a diagnostic outcome that matched the child's previous diagnosis. The experience of the stakeholder's study (Nazneen, Matthews et al., 2015) reported very positive findings from both the parents and clinicians. Videos recorded at home were particularly useful in that they allowed the clinicians to observe evidence of naturalistic behaviours in children, something which is not possible otherwise in clinical settings. Clinicians reported that a complete assessment of one child would 
take one hour. They felt that the children with classical ASD symptoms would be easily and successfully diagnosed using NODA, but that children with different presentations would need an additional assessment. It was suggested that NODA could be an effective early screener in such cases (Nazneen, Matthews et al., 2015). Cases where NODA would be most useful were identified as being with rural families and divorced or separated families, as both parents could upload and share videos separately. The studies examining the usability of NODA were positive overall, with 40 out of 44 parents feeling that they could use the system and that it would improve the time taken to obtain a diagnosis.

A study by Smith et al. (2017) included 51 participants (full sample) and compared NODA (remote approach) with the gold standard (in person assessment) for 40 families with ASD children (subsample) and 11 with non-ASD children. There was substantial agreement in the diagnosis, with a diagnostic agreement of $88.2 \%(n=45)$ in the full sample and $85 \%(\mathrm{n}=34)$ in the subsample. The sensitivity was $84.5 \%$ and the specificity was between $94.4 \%$ (full sample) and $85.7 \%$ (subsample). The Kappa coefficients for inter-rater reliability showed $85 \%$ to $90 \%$ accuracy between the raters. Therefore, NODA was felt to be a reliable system that performed well compared to the gold standard. It was further stated that NODA is not aimed at removing the need for upcoming assessments but that it is aimed to accelerate the pathway to early interventions and treatment. Collectively, these studies concluded that NODA has the potential to improve the efficiency of the ASD diagnostic process. 


\subsection{Discussion}

The literature review found 10 papers (5 independent research teams) that used telehealth in the diagnosis of ASD. Eight of the studies were rated to be of "fair" quality, whereas two studies were rated "poor", using the NHLBI and CASP assessment tools. Most of the studies had small sample sizes (with the range of samples being 10 to 45) with descriptive analysis, including a survey design and qualitative experience of the systems. Therefore, it seems obvious that more high-quality studies are needed in the future, to shed more light on this important area. Referring back to the Objective (1) of the thesis, there were 2 methods of using telehealth in the diagnosis of ASD; (1) Real-Time method e.g. videoconferencing that enables a range of health professionals in different areas to meet in real time with the family to assess the child or adult, thus tackling the issues associated with geographical spread. This means that access to specialists might be better facilitated. (2) A Store-and-Forward method with parents/carers using a NODA system to upload videos of child's behaviours and interactions, in order to enable the clinicians making an assessment remotely and sharing with parents/carers or other health professionals. This means that health professionals could conduct remote observations in the family home, thereby eliminating the need for the family to travel and also enabling observations to be made in more naturalistic settings. Nearly all the systems identified are designed to enable the clinicians to undertake an assessment for ASD, e.g. allowing the ability to score or mark videos, the ability to zoom in or out to observe facial expressions. This review of the literature found evidence that telehealth methods allow for collaboration and the sharing of experiences between the family, education establishments, ASD experts and the university medical centres. Furthermore, they can be regarded as equal to face to face methods in terms of satisfaction for the patient, family and clinician. They enable rural families to be seen at lower cost and reduce the time required to make a diagnosis, particularly for those with more obvious symptoms of autism, where there is in good agreement in terms of the diagnosis compared to the face to face methods. The findings from the review suggest that telehealth can potentially improve the efficiency of the diagnosis process for ASD. 
All of the studies originated in the USA. However, implementing telehealth systems may facilitate the ASD assessment and diagnosis in other settings with different healthcare systems. In the UK, the multi-disciplinary team approach is one of the assessment methods used for ASD diagnosis, although it is not available in all areas (National Autistic Society, 2018b; National Health Service, 2019). Research has shown that it is difficult to get a multi-disciplinary team together, especially when they span different disciplines, such as education and health (Hurt et al., 2019). Therefore, telehealth may provide support to a specialist ASD team, allowing them to meet remotely and provide a diagnosis, regardless of their locations. Moreover, there are critical consequences of a delay in diagnosis as there has been reported a delay of approximately 3.5 years between initially seeking help and receiving a confirmed diagnosis of ASD (Crane et al., 2015). Telehealth technology may help to provide a timely diagnosis and, although it may not be appropriate for complicated cases, it could be used as a screening tool for ASD. Consequently, this may increase the opportunity for early intervention as well as reducing stress levels for individuals and their families.

\subsubsection{The strengths, limitations and future direction of this review}

The strength of this review was in its comprehensive search strategy, intending to find all published studies regardless of method and research quality in order to broadly find out what is known about using telehealth for ASD diagnostic assessment. As part of this thesis, this was important to be reviewed in such approach, particularly before consideration was given to parents/carers perceptions towards the remote diagnostic assessment. However, there were some inconsistencies in the terminology across the studies and a general lack of precision in the descriptions of the telehealth technologies. Furthermore, this review only included papers that were published or written in English. This field is very new and only early design evaluations of the software and the clinician and family experiences of the system were available. There is a need for future high-quality studies using large sample sizes. More clarity in research questions, adequate details about sampling and inclusion and exclusion criteria, more clarity in the rigor of data analysis and reporting of ethics should also be taken in the consideration. However, at this early stage, telehealth appears to offer the 
potential to improve the time required to obtain a diagnosis of ASD. To date, the evidence suggests that the Real-Time and Store-and-Forward methods such as videoconferencing and NODA system: (1) are acceptable to both the families and clinicians; (2) have good diagnostic accuracy; (3) enable families from a wider area to access professionals; (3) reduce costs for accessing care; (4) enable natural behaviours in the home setting to be observed; and (5) may enable both parents in divorced families to contribute to the diagnostic process. This approach of diagnosis is complementary to other developments in this field such as the DAWBA (Development and Well-Being Assessment), which is a computerised assessment using the Strengths and Difficulties Questionnaire to reduce waiting times and inappropriate referrals (Goodman, Ford, Richards, Gatward, \& Meltzer, 2000). The use of the existing telehealth methods could also greatly improve the validity of diagnosis in trials of interventions for ASD, by enabling the same team of health professionals to see every patient in the trial, regardless of location. Finally, the use of telehealth methods seems to be most helpful for those who have clear ASD characteristics as opposed to those whose characteristics may be borderline, and may be useful for the diagnosis of adults with ASD.

\subsubsection{The implications of the findings for practice}

This is the first step in examining the potential of using telehealth in conjunction with the current practices, in order to improve the time required to obtain a diagnosis for people with ASD. Larger studies with a randomised design will be needed to confirm that telehealth can improve the diagnosis times.

\subsection{Conclusion}

Early evidence about the use of telehealth for ASD diagnosis suggests that the systems available to date are feasible and acceptable to families and clinicians. These systems have the potential to improve the time required to obtain a diagnosis for the families and people with ASD, but do warrant further study. 


\subsection{Findings to be taken forward}

This scoping review suggests that the telehealth ASD diagnostic approaches available to date are feasible and acceptable to families and clinicians. However, findings from this nascent body of literature indicate that, to date, no study has examined the factors based on the theoretical foundation that drives the acceptance of telehealth for diagnostic assessment, by the parents of children with ASD. Therefore, after exploring the parents/carer experience of the diagnostic process of ASD (Study 2), Study (3) aimed to fill this knowledge gap and build upon prior work, by investigating the driver factors of the acceptance of telehealth amongst parents/carers of children with ASD.

As we have seen, this review of the literature found there to be two methods of using telehealth: (a) Real-Time method and (b) A Store-and-Forward method. However, as this thesis is focusing on the parents/carers of children with ASD perceptions, the NODA system was chosen from amongst other telehealth technologies, with the aim of examining the factors that influence the degree to which parents/carers accept the use of this system for diagnostic assessment. This choice was on account of the fact that Store-and-Forward telehealth approaches such as NODA allows parents to easily record videos of the behaviour of their children in their home. This can be done at any time suitable for them, as it does not need parents and diagnosticians to both be available at the same time (Nazneen, Oberleitner, \& Reischl, 2016; Smith et al., 2017). Alternatively, with Real-Time telehealth approaches such as videoconferencing, parents may be required to travel to a diagnostic centre or regional clinic to access the technology and where the setting is prepared with different probes (e.g., toys, books) in order to engage the child, according to the instructions from the diagnostician in the remote location (Nazneen et al., 2016). Home recording of the videos over the course of day-to-day activities may also help in observing the natural expressions of the child's behaviour within their natural environment, something which is essential in order to obtain a complete and accurate diagnostic assessment (Matson, 2008; McLeod, Jensen-Doss, \& Ollendick, 2013; Nazneen, Rozga et al., 2015; Smith et al., 2017). Hence, the NODA system was selected as the preferred method of telehealth technology for this research, in order to determine the predictors of parents/carers acceptance to use such a system in Wales. 


\subsection{Summary}

This chapter presented a scoping review that was aimed to examine those telehealth approaches which have been used in the diagnosis and assessment of ASD in children and adults, whether they are feasible and acceptable, and how they compare with face to face diagnosis and assessment methods. A search for all peer-reviewed articles dating from 2000 to 2019, combining the terms of autism and telehealth was conducted. A total of 10 studies were identified for inclusion in the review. This review of the literature found there to be two methods of using telehealth: (a) Real-Time method e.g. videoconferencing (b) A Store-and-Forward method e.g. Naturalistic Observation Diagnostic Assessment (NODA) system. The findings were positive, with an apparently high level of agreement in terms of the diagnosis between remote methods and in-person methods, and with high levels of acceptability among both families and clinicians. This field is in the very early stages but the findings suggest that there is potential for telehealth methods to improve access to assessment and diagnosis of ASD, particularly for those with clear autism traits. Larger randomised controlled trials of this technology are warranted. The next chapter will discuss the most influential technology acceptance theories and models, including any limitations. It will end with justification for the selection of the Unified Theory of Acceptance and Use of Technology (UTAUT) as a base model to study the degree to which parents/carers in Wales will accept the use of NODA system for ASD diagnostic assessment. 


\section{Chapter 4: Technology Acceptance Models and Theories}

\subsection{Introduction}

This chapter covers two main areas. Firstly, the most influential technology acceptance models and theories are described. Secondly, the model that was used to explore parents/carers acceptance of using the NODA system for ASD diagnostic assessment is reviewed, including the reasons for choosing this particular model from amongst the various technology acceptance models.

\subsection{Technology acceptance}

Research has shown that acceptance by the user is imperative for the development of any new technology (Davis, 1993; Taherdoost, 2018). According to Dillon (2001), technology acceptance is about the willingness within a user group to use information technology for its intended purpose. User acceptance of technology has also been referred to as the psychological state of a person with regard to his or her voluntary or intended use of a specific technology (Chau \& Hu, 2001). Policymakers and researchers who are trialling a new information system need to understand the degree of users acceptance and the issues that may impact upon their decision whether to use a system or not (Garavand et al., 2016; Taherdoost, 2018). This will provide noteworthy help in the successful technologies' implementation (Garavand et al., 2016; Momani, Jamous, \& Hilles, 2017; Taherdoost, 2018). Technical and nontechnical factors should be clearly identified before the implementation of any such system (Garavand et al., 2016). Lack of user acceptance is a main barrier to the success of new information systems (Davis, 1993; Dillon \& Morris, 1996). Therefore, user acceptance is considered to be the essential factor that can determine either the success or failure of any information system schemes, including telehealth (Davis, 1993; Hu et al., 1999). To ensure a successful implementation of telehealth, researchers are seeking a deeper understanding of the predictors that make telehealth technology acceptable for the users in different contexts (Cimperman, Brenčič, \& Trkman, 2016; Hoque \& Sorwar, 2017; Kim et al., 2018; Kowitlawakul, 2011). 
Numerous theoretical models attempt to describe an individual's decision to accept new technology and introduce factors that might affect that acceptance. In this chapter, we review the most widely used technology acceptance theories and models: Theory of Reasoned Action (TRA) (Ajzen \& Fishbein, 1980; Fishbein \& Ajzen, 1975), Theory of Planned Behaviour (TPB) (Ajzen, 1985, 1991), Diffusion of Innovation Theory (DOI) (Rogers, 1995, 2003), Technology Acceptance Model (TAM) (Davis, 1989; Davis, Bagozzi, \& Warshaw, 1989), and Unified Theory of Acceptance and Use of Technology (UTAUT) (Venkatesh et al., 2003). Recent systematic review (Harst, Lantzsch, \& Scheibe, 2019) revealed that these theories and models are applicable to be used on telehealth adoption. This review has also reported the frequencies of these theories and models that were used to predict end-user acceptance of telehealth within the included studies (see Table 4-1).

Table 4-1: Frequency of theories and models used to predict end-user acceptance of telehealth use (Harst et al., 2019)

\begin{tabular}{lcc}
\multicolumn{1}{c}{ Model/Theory } & Frequency of use & Year \\
\hline $\begin{array}{l}\text { Theory of Reasoned Action } \\
\text { (TRA) }\end{array}$ & 2 & 2011,2016 \\
$\begin{array}{l}\text { Theory of Planned Behaviour } \\
\text { (TPB) }\end{array}$ & 2 & 2014,2016 \\
$\begin{array}{l}\text { Diffusion of Innovation } \\
\text { Theory (DOI) }\end{array}$ & 3 & $2005,2012,2015$ \\
$\begin{array}{l}\text { Technology Acceptance } \\
\text { Model (TAM); (TAM2) }\end{array}$ & 11,2 & $2009,2010,2011$, \\
& & $2012(2), 2014,2016$, \\
& & $2017(3), 2018 ; 2010$, \\
$\begin{array}{l}\text { Unified Theory of Acceptance } \\
\text { and Use of Technology }\end{array}$ & 9 & 2017 \\
(UTAUT) & & $2013(2), 2015(4), 2016$, \\
\hline
\end{tabular}

Figure 4-1 presents the basic concept of the user acceptance models and theories, in which the intention to use any technology as a predictor of usage behaviour is significant, and has been entrenched in many information systems (Ajzen, 1991; Sheppard, Hartwick, \& Warshaw, 1988; Taylor \& Todd, 1995). The next section discusses the five theories and models. 


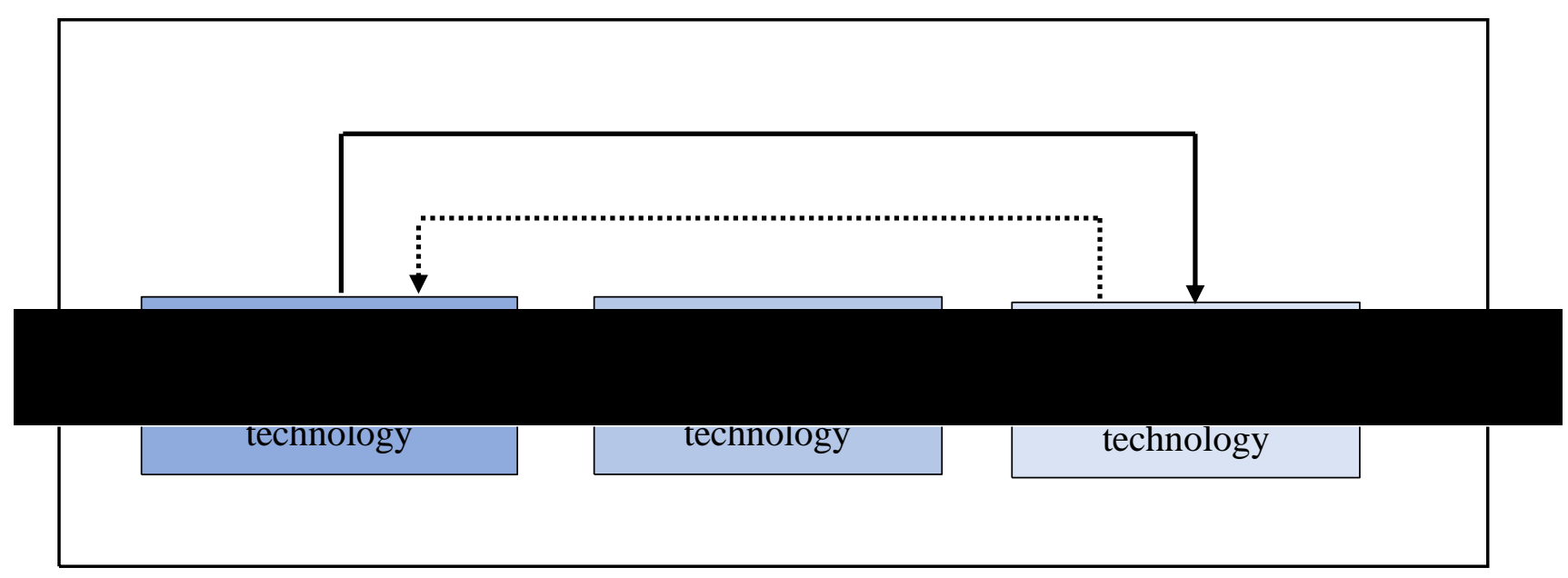

Figure 4-1: Basic concept underlying user acceptance models and theories (Venkatesh, Morris, Davis, \& Davis, 2003)

\subsection{Technology acceptance models and theories}

\subsubsection{Diffusion of Innovation Theory (DOI)}

The Diffusion of Innovation Theory (DOI) has been used since the 1960's (Rogers, 1995). The theory helped to explain various studies on innovation diffusion, from agricultural studies to information systems (Greenhalgh, Robert, Bate, Macfarlane, \& Kyriakidou, 2005; Moore \& Benbasat, 1991; Rogers, 2003). Diffusion of innovation is identified as occurring within a five-step process (Rogers, 2003), namely that the individual passes from knowledge about the innovation, to attitude regarding the innovation, to a decision of either adoption or rejection, to implementation of the innovation, and to confirmation of the decision (see Figure 4-2). The five-step innovation decision process is as follows:

- Knowledge is obtained when the individual discovers the presence of an innovation, and gains more understanding of the innovation.

- Persuasion takes a place when the individual forms an attitude about the innovation, either favourable or unfavourable.

- Decision occurs when the individual undertakes the activities that lead to a decision, whether that be accepting or rejecting the innovation. 
- Implementation takes place when the individual uses the innovation.

- Confirmation occurs when the individual tries to identify means of strengthening the decision that he or she has already made about the innovation. However, the previous decision could be reserved if they are exposed to conflicting message regarding the innovation, meaning either rejection of the adopted innovation or replacement with a better innovation.

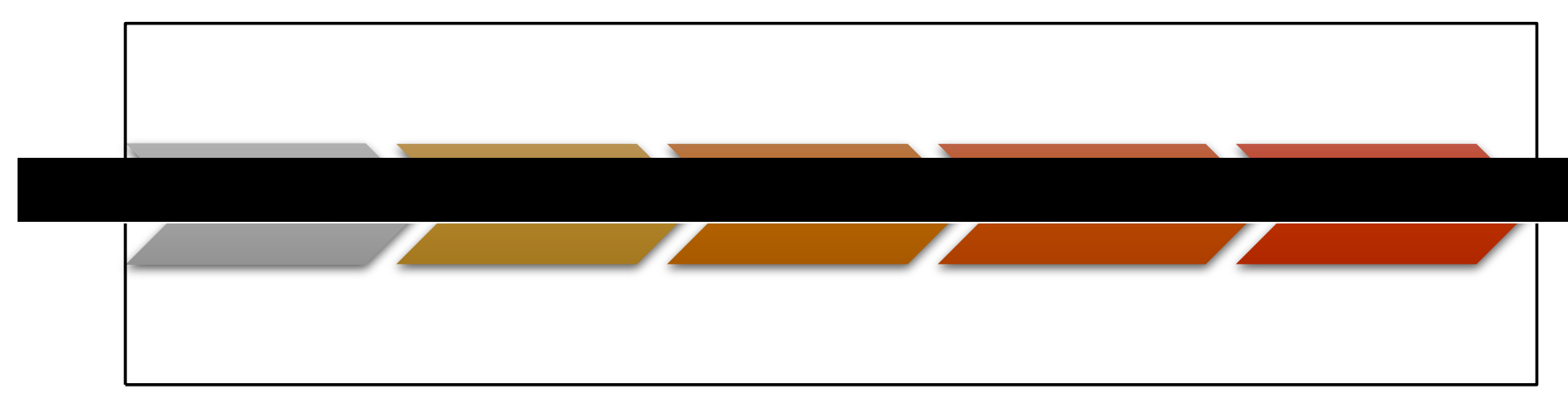

Figure 4-2: Innovation Decision Process (Rogers, 2003)

It has been suggested that DOI focuses more on the organisational characteristics, environmental aspects and system features. However, compared to other acceptance models and theories, it has less power in explanatory and less practicable for outcomes prediction (Taherdoost, 2018).

\subsubsection{Theory of Reasoned Action (TRA)}

The Theory of Reasoned Action (TRA) is one of the basic theories in psychology (Ajzen \& Fishbein, 1980; Fishbein \& Ajzen, 1975). TRA is very general model that has been designed to predict and explain any individual's behaviour (Ajzen \& Fishbein, 1980). According to the TRA, a person's actual behaviour is determined by his or her behavioural intention to perform the behaviour, and behavioural intention is determined by the person's attitude toward behaviour (A) and subjective norm (SN) (see Figure 4-3) (Ajzen \& Fishbein, 1980; Fishbein \& Ajzen, 1975). TRA is an influential theory of individual behaviour that has been used in predicting and understanding behaviours across different domains such as sociology, information technologies and marketing (Davis et al., 1989; Venkatesh et al., 2003). A meta- 
analysis that was conducted by Sheppard et al. (1988) found strong support for the predictive utility of the TRA. However, TRA has been criticised. In particular, it has shown that TRA failed to predict behaviour of individuals in situations in which they have an incomplete volitional control (Ajzen, 1991). Volitional control (the ability to decide whether or not to perform the behaviour) is predicted to moderate the relation between intention and behaviour such that the impact of intention on behaviour is stronger when control is high rather than low. That is, people tend to act in accordance with their intentions when they have control over the performance of a behaviour. When the behaviour is not completely under volitional control, evaluating insights of behavioural control can help improve prediction. For example, when parents/carers have control over performance of a using remote ASD diagnostic technology, intention by itself should permit good prediction. Furthermore, TRA does not indicate the beliefs that are operative for a specific behaviour (Davis et al., 1989).

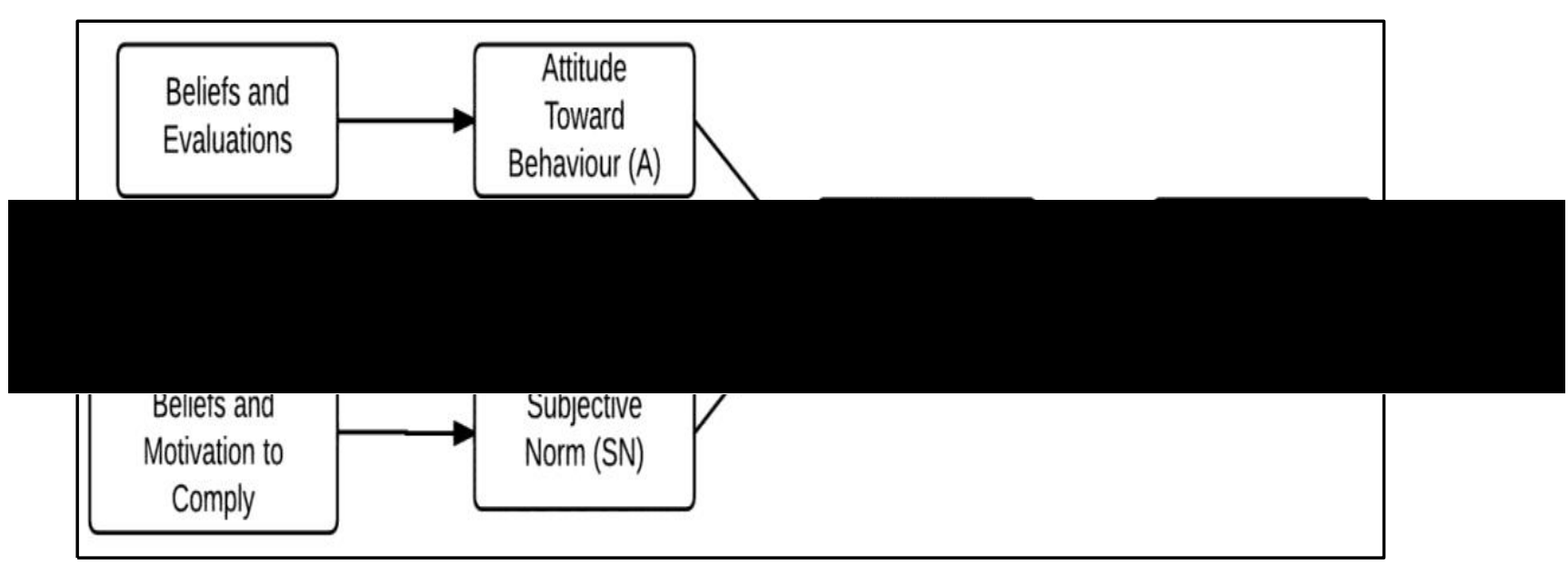

Figure 4-3: Theory of Reasoned Action (TRA) (Ajzen \& Fishbein, 1980; Fishbein \& Ajzen, 1975) 


\subsubsection{Theory of Planned Behaviour (TPB)}

The Theory of Planned Behaviour (TPB) was developed by Ajzen (1985) as an extension of the Theory of Reasoned Action (TRA). In the TRA model, behavioural intention is determined by two constructs; attitude toward behaviour and subjective norm whilst Ajzan (1985) added an additional construct in TPB, called perceived behavioural control (PBC), in order to overcome a weakness of the TRA model, namely that it lacked the social factors that may influence the individual's behaviour (see Figure 4-4). Similar to TRA, TPB was used by numerous studies in order to predict intention as well as behaviour in a variety of settings (Ajzen, 1991). However, it has been indicated that constructs of TPB (attitude toward behaviour, subjective norms, and perceived behavioural control) are insufficient to predict the behavioural intention of an individual (Ajzen \& Fishbein, 2005).

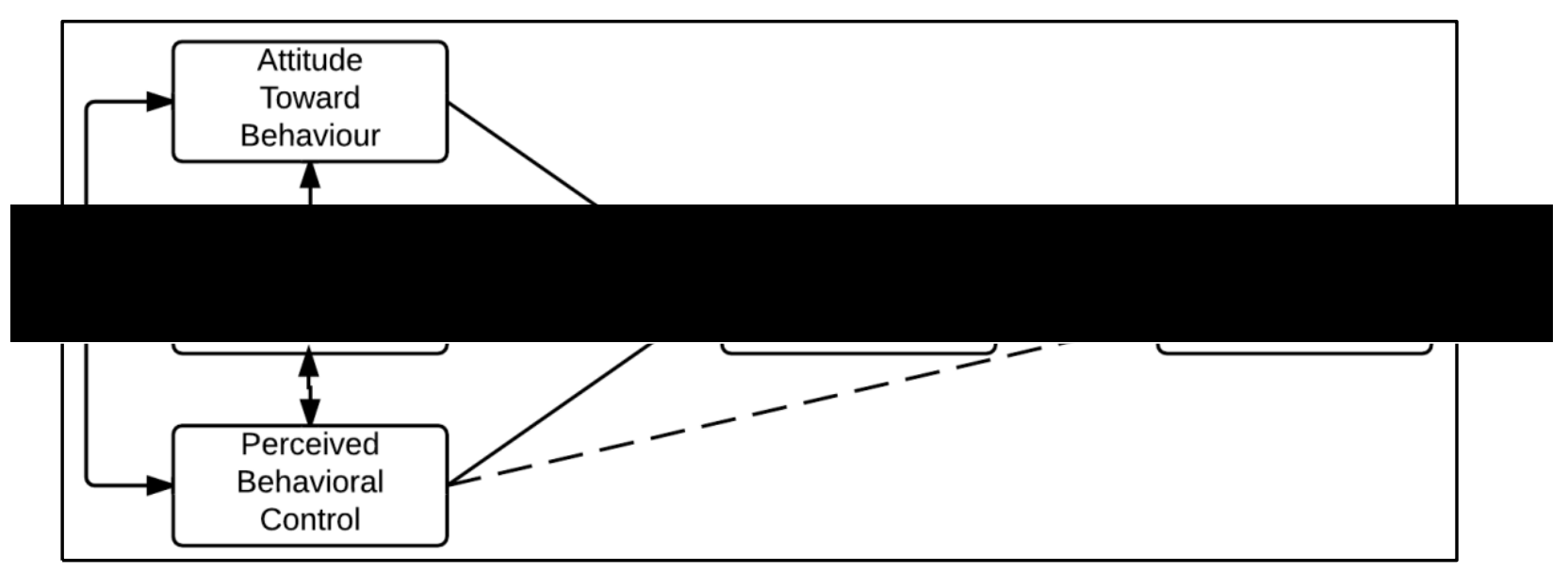

Figure 4-4: Theory of Planned Behaviour (TPB) (Ajzen, 1985)

\subsubsection{Technology Acceptance Model (TAM)}

The Technology Acceptance Model (TAM), developed by Davis (1989), was adapted from the Theory of Reasoned Action (TRA), especially designed for modelling user acceptance of information systems. The main purpose of the model is to explain the determinants of computer acceptance, with the added ability of providing an explanation of individual behaviour across different populations in relation to a wideranging of technologies (Davis, 1989). Similar to TRA, TAM suggests that computer usage is determined by behavioural intention. However, unlike TRA, TAM doesn't include the subjective norm (SN) as a determinant of intention. Furthermore, unlike 
the TRA that is designed to describe any human behaviour (Ajzen \& Fishbein, 1980), TAM is considered more specific, intended to be applied to behaviour associated to computer usage (Davis et al., 1989).

TAM speculates that two constructs, namely perceived ease of use (PEOU) and perceived usefulness (PU), together help to determine a person's behavioural intention to use a system (Venkatesh \& Davis, 2000). Perceived ease of use (PEOU) refers to "the degree to which a person believes that using a particular system would be free of effort" (Davis, 1989, p. 320), while perceived usefulness is defined as "the degree to which a person believes that using a particular system would enhance his or her job performance" (Davis, 1989, p. 320). Many empirical studies reported that behavioural intention is a good predictor of actual usage of a technology (Davis et al., 1989; Venkatesh \& Davis, 2000). The TAM model is shown below in Figure 4-5.

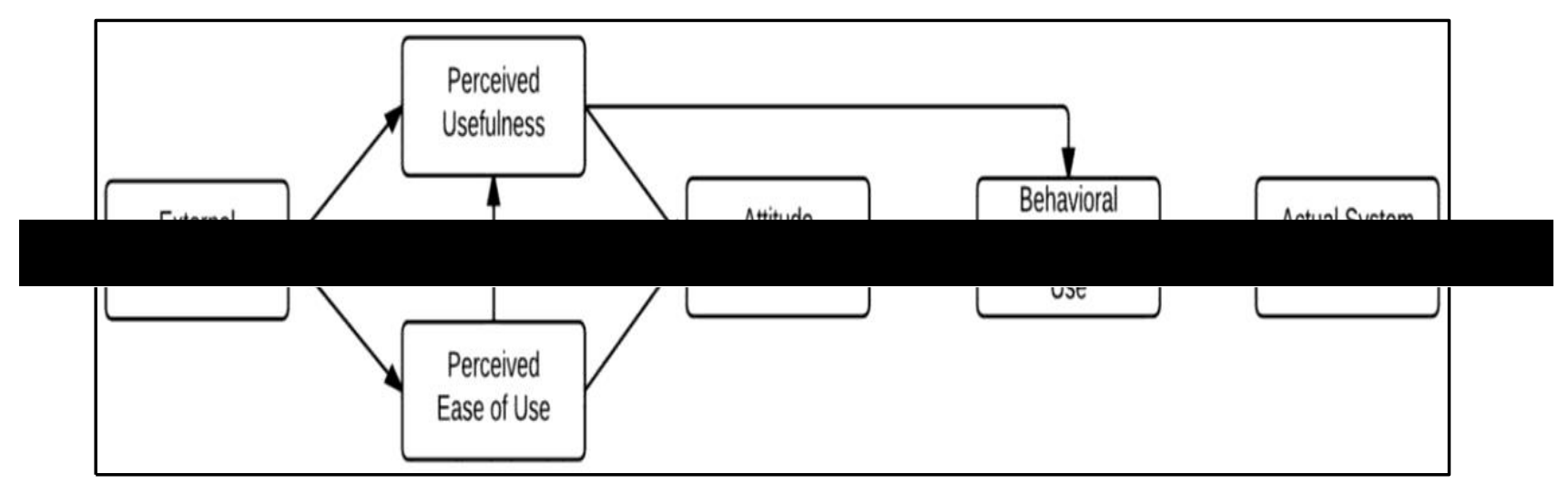

Figure 4-5: Technology Acceptance Model (TAM) (Davis, 1989)

TAM is an influential and robust model with which to predict user acceptance (Venkatesh \& Davis, 2000). It has been used in different domains such as healthcare, education and information management to assess user acceptance, identifying the range of usage behaviours and intentions (Esmaeilzadeh, Sambasivan, Kumar, \& Nezakhati, 2011; Ghazizadeh, Lee, \& Boyle, 2012; Granić \& Marangunić, 2019; Opoku \& Francis, 2020). It has also been found that TAM can explain around 40\%$60 \%$ of the variance in behavioural intention (Tang \& Chen, 2011; Venkatesh et al., 2003). 
Although TAM is widely used by different studies, it has previously been criticised. Although perceived ease of use and usefulness are important factors, predicting individual attitudes towards technology using only these two factors ignores several other possible factors (Straub, 2009). TAM lacks the individual differences. Individual differences are those user factors that involve characteristics such as demographic and personality variables, in addition to the situational variables that may account for variances attributable to circumstances (Agarwal \& Prasad, 1999). The original TAM does not consider such factors including age, gender, training and prior experience, that might influence user attitude, which in turn might influence the user intention to use the technology (Agarwal \& Prasad, 1999; Ajibade, 2018; Straub, 2009). Moreover, Ghazizadeh et al., (2012) indicate that the original TAM did not include any social and control factors, as used in the Theory of Planned Behaviour (TPB), such as subjective norms and perceived behavioural control.

Venkatesh and Davis (2000) suggested an extension of TAM, referred to as TAM2 (see Figure 4-6) in order to improve the adaptability of the original TAM (Venkatesh \& Davis, 2000). The model was tested using longitudinal data collected from four different systems at four different organisations $(n=156)$ (Tang \& Chen, 2011; Venkatesh \& Davis, 2000). TAM2 includes additional constructs: subjective norm, voluntariness, image, experience, job relevance, output quality and result demonstrability. TAM2 has been used to understand acceptance of using technology amongst different populations. For example, Zhang, Cocosila and Archer (2010) examined adoption factors of mobile information technology by homecare nurses. Dou et al. (2017) also intended to explore patients' acceptance of using smartphone health technology for chronic disease management. The extended model accounted for $40 \%$ $-60 \%$ of the variance in usefulness and $34 \%-52 \%$ of the variance in intention to use. Although these results are useful, further research is still required to validate the model, involving larger sample sizes in different contexts. 


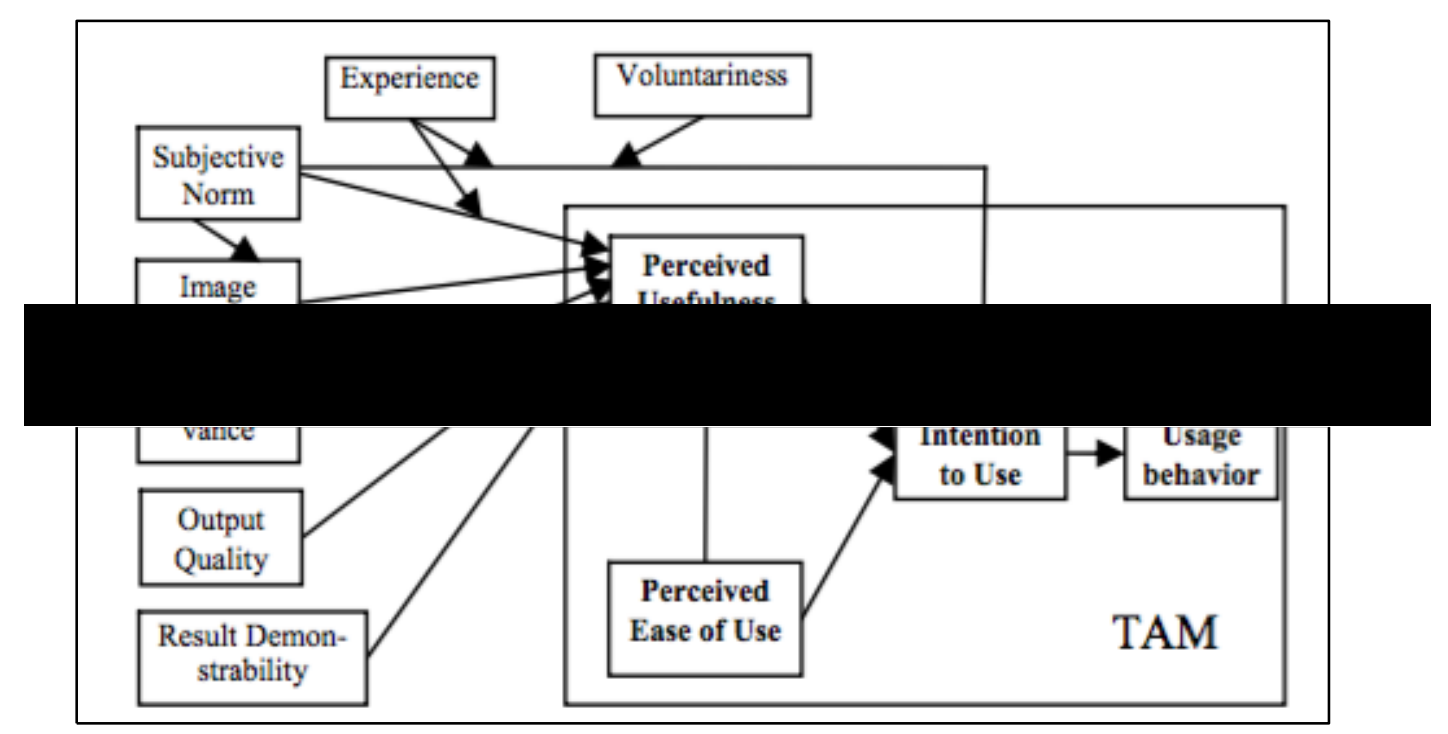

Figure 4-6: Technology Acceptance Model 2 (TAM2) (Venkatesh \& Davis, 2000)

TAM2 has also been extended to TAM3 (see Figure 4-7) (Venkatesh \& Bala, 2008). The model was empirically tested over a 5-month period with four points of measurements with a sample of 156 at every time period, using longitudinal data collected from four different organisations. The findings explained $53 \%$ of the variance in intention to use (Tang \& Chen, 2011; Venkatesh \& Bala, 2008). However, TAM3 was mostly used to explore how managers make decisions regarding new interventions, which can then result in higher acceptance levels and utilisation of Information Technology (IT) applications (Venkatesh \& Bala, 2008). The next section describes the Unified Theory of Acceptance and Use of Technology (UTAUT) model. 


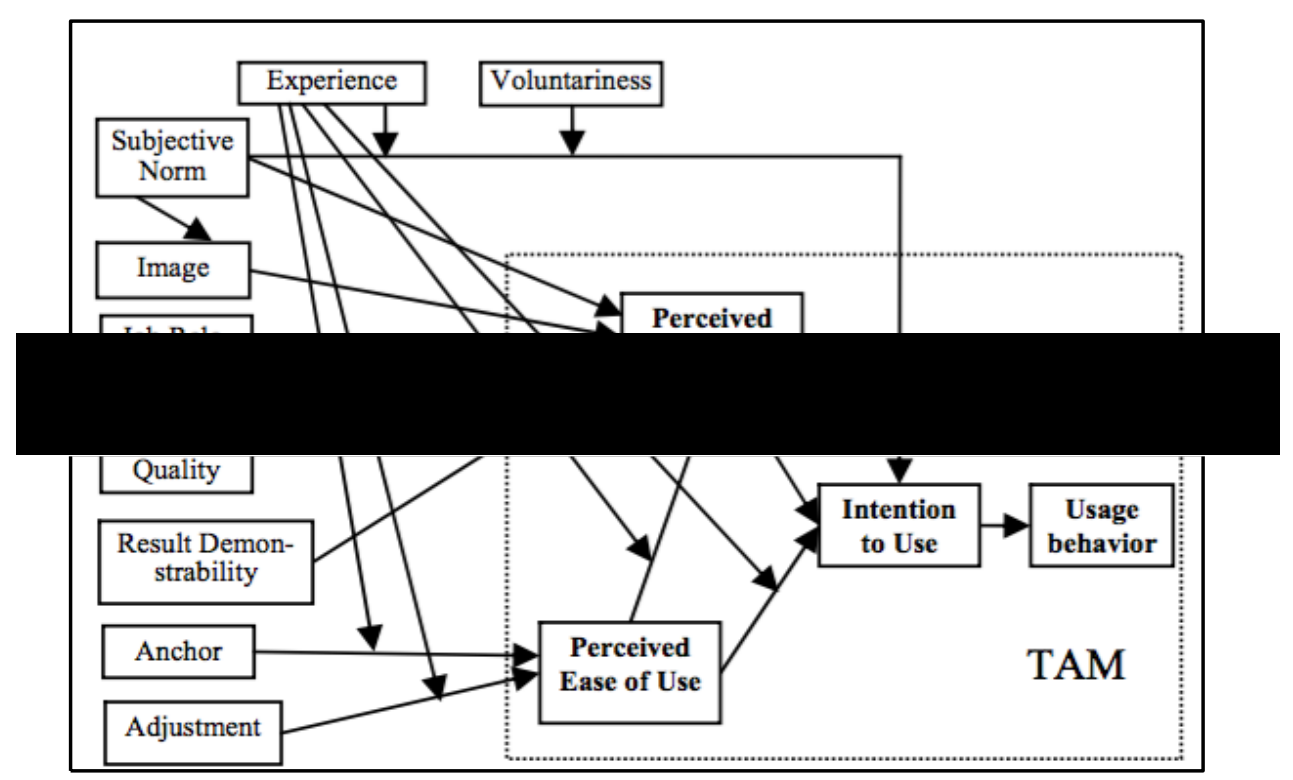

Figure 4-7: Technology Acceptance Model 3(TAM3) (Venkatesh \& Bala, 2008)

\subsubsection{Unified Theory of Acceptance and Use of Technology (UTAUT)}

The Unified Theory of Acceptance and Use of Technology (UTAUT) is a model that was developed by Venkatesh et al. (2003) and based on combining the constructs of eight established research models, including:

- Diffusion of Innovation Theory (DOI)

- Theory of Reasoned Action (TRA)

- Theory of Planned Behaviour (TPB)

- Technology Acceptance Model (TAM)

- Motivational Model (MM)

- Combined Theory of Planned Behaviour - Technology Acceptance Model (CTAMTPB)

- Model of Personal Computer Utilization (MPCU)

- Social Cognitive Theory (SCT). 
The Unified Theory of Acceptance and the Use of Technology has several advantages over other models. The UTAUT is the most comprehensive theory amongst the technology acceptance models (Venkatesh et al., 2003). In order to overcome deficiencies, it incorporates various determinants from several models of individual acceptance (see Table 4-2) (Venkatesh et al., 2003). The UTAUT was specifically developed to explain the behaviour of organisations or individuals relating to their use of information systems (Venkatesh et al., 2003). A review indicated that UTAUT has been extensively used as theoretical framework in technology acceptance research, used in conducting studies of individual behavioural intention as well as behaviour (Williams, Rana, \& Dwivedi, 2015). UTAUT was also being discussed with regard to different technologies such as the Internet, Hospital Information Systems, Web Sites, and Mobile Technology, and focusing on different user groups including professionals, patients, students and general users to understand the degree of their acceptance (Williams et al., 2015).

Table 4-2: The determinants of individual acceptance models and theories. Adapted from (Venkatesh et al., 2003)

\begin{tabular}{|c|c|c|}
\hline Models/Theories & $\begin{array}{c}\text { Core } \\
\text { Constructs } \\
\end{array}$ & Definitions \\
\hline \multirow[t]{2}{*}{$\begin{array}{l}\text { Theory of } \\
\text { Reasoned Action } \\
\text { (TRA) }\end{array}$} & $\begin{array}{l}\text { Attitudes } \\
\text { towards the } \\
\text { behaviour }\end{array}$ & $\begin{array}{l}\text { "an individual's positive or negative feelings } \\
\text { about performing the target behaviour" (Fishbein } \\
\text { \& Ajzen, 1975, p. 216). }\end{array}$ \\
\hline & $\begin{array}{l}\text { Subjective } \\
\text { Norm }\end{array}$ & $\begin{array}{l}\text { "the person's perception that most people who are } \\
\text { important to him think he should or should not } \\
\text { perform the behaviour in question" (Fishbein \& } \\
\text { Ajzen, 1975, p. 302). }\end{array}$ \\
\hline \multirow[t]{2}{*}{$\begin{array}{l}\text { Technology } \\
\text { Acceptance } \\
\text { Model (TAM) }\end{array}$} & $\begin{array}{l}\text { Perceived } \\
\text { Usefulness }\end{array}$ & $\begin{array}{l}\text { "the degree to which a person believes that using } \\
\text { a particular system would enhance his or her job } \\
\text { performance" (Davis, 1989, p. 320). }\end{array}$ \\
\hline & $\begin{array}{l}\text { Perceived Ease } \\
\text { of Use }\end{array}$ & $\begin{array}{l}\text { "the degree to which a person believes that using } \\
\text { a particular system would be free of effort" } \\
\text { (Davis, } 1989, \text { p. } 320 \text { ). }\end{array}$ \\
\hline \multirow[t]{2}{*}{$\begin{array}{l}\text { Motivational } \\
\text { Model (MM) }\end{array}$} & $\begin{array}{l}\text { Extrinsic } \\
\text { Motivation }\end{array}$ & $\begin{array}{l}\text { "the perception that users will want to perform an } \\
\text { activity because it is perceived to be instrumental } \\
\text { in achieving valued outcomes that are distinct } \\
\text { from the activity itself" (Davis, Bagozzi, \& } \\
\text { Warshaw, 1992, p. 1112). }\end{array}$ \\
\hline & $\begin{array}{l}\text { Intrinsic } \\
\text { Motivation }\end{array}$ & $\begin{array}{l}\text { "the perception that users will want to perform an } \\
\text { activity for no apparent reinforcement other than } \\
\text { the process of performing the activity per se" } \\
\text { (Davis et al., 1992, p. 1112). }\end{array}$ \\
\hline
\end{tabular}




\begin{tabular}{|c|c|c|}
\hline \multirow[t]{3}{*}{$\begin{array}{l}\text { Theory of } \\
\text { Planned } \\
\text { Behaviour(TPB) }\end{array}$} & $\begin{array}{l}\text { Attitude } \\
\text { towards } \\
\text { behaviour }\end{array}$ & Adapted from TRA \\
\hline & Subject Norm & Adapted from TRA \\
\hline & $\begin{array}{l}\text { Perceived } \\
\text { Behavioural } \\
\text { Control }\end{array}$ & $\begin{array}{l}\text { "the perceived ease or difficulty of performing } \\
\text { the behaviour" (Ajzen, 1991, p. 188). }\end{array}$ \\
\hline \multirow{4}{*}{$\begin{array}{l}\text { Combined } \\
\text { Technology } \\
\text { Acceptance } \\
\text { Model and } \\
\text { Theory of } \\
\text { Planned } \\
\text { Behaviour (C- } \\
\text { TAM-TPB) }\end{array}$} & $\begin{array}{l}\text { Attitude } \\
\text { Toward } \\
\text { Behaviour }\end{array}$ & Adapted from TRA/TPB \\
\hline & $\begin{array}{l}\text { Subjective } \\
\text { Norm }\end{array}$ & Adapted from TRA/TPB \\
\hline & $\begin{array}{l}\text { Perceived } \\
\text { Behavioural } \\
\text { Control }\end{array}$ & Adapted from TRA/TPB \\
\hline & $\begin{array}{l}\text { Perceived } \\
\text { Usefulness }\end{array}$ & Adapted from TAM \\
\hline \multirow{6}{*}{$\begin{array}{l}\text { Model of } \\
\text { Personal } \\
\text { Computer } \\
\text { Utilization } \\
\text { (MPCU) }\end{array}$} & Job-fit & $\begin{array}{l}\text { "the extent to which an individual believes that } \\
\text { using [a technology] can enhance the } \\
\text { performance of his or her job" (Thompson, } \\
\text { Higgins, \& Howell, 1991, p. 129). }\end{array}$ \\
\hline & Complexity & $\begin{array}{l}\text { "the degree to which an innovation is perceived } \\
\text { as relatively difficult to understand and use } \\
\text { (Thompson et al., 1991, p. 128). }\end{array}$ \\
\hline & $\begin{array}{l}\text { Long-term } \\
\text { Consequences }\end{array}$ & $\begin{array}{l}\text { "outcomes that have a pay-off in the future" } \\
\text { (Thompson et al., 1991, p. 129) }\end{array}$ \\
\hline & $\begin{array}{l}\text { Affect Towards } \\
\text { Use }\end{array}$ & $\begin{array}{l}\text { "feelings of joy, elation, or pleasure, or } \\
\text { depression, disgust, displeasure, or hate } \\
\text { associated by an individual with a particular act" } \\
\text { (Thompson et al., 1991, p. 127). }\end{array}$ \\
\hline & Social Factors & $\begin{array}{l}\text { "the individual's internalization of the reference } \\
\text { group's subjective culture and specific } \\
\text { interpersonal agreements that the individual has } \\
\text { made with others, in specific social situations" } \\
\text { (Thompson et al., 1991, p. 126). }\end{array}$ \\
\hline & $\begin{array}{l}\text { Facilitating } \\
\text { Conditions }\end{array}$ & $\begin{array}{l}\text { "objective factors in the environment that } \\
\text { observers agree make an act easy to accomplish" } \\
\text { (Venkatesh et al., 2003, p. 430). }\end{array}$ \\
\hline \multirow[t]{5}{*}{$\begin{array}{l}\text { Diffusion of } \\
\text { Innovation } \\
\text { Theory (DOI) }\end{array}$} & $\begin{array}{l}\text { Relative } \\
\text { Advantage }\end{array}$ & $\begin{array}{l}\text { "the degree to which an innovation is perceived } \\
\text { as being better than its precursor" (Moore \& } \\
\text { Benbasat, 1991, p. 195). }\end{array}$ \\
\hline & Ease of Use & $\begin{array}{l}\text { "the degree to which an innovation is perceived } \\
\text { as being difficult to use" (Moore \& Benbasat, } \\
\text { 1991, p. 195). }\end{array}$ \\
\hline & Image & $\begin{array}{l}\text { "the degree to which use of an innovation is } \\
\text { perceived to enhance one's image or status in } \\
\text { one's social system" (Moore \& Benbasat, 1991, } \\
\text { p. 185). }\end{array}$ \\
\hline & Visibility & $\begin{array}{l}\text { "the degree to which one can see others using the } \\
\text { system in the organisation" (Venkatesh et al., } \\
2003, \text { p. } 431 \text { ) }\end{array}$ \\
\hline & Compatibility & $\begin{array}{l}\text { "the degree to which an innovation is perceived } \\
\text { as being consistent with the existing values, }\end{array}$ \\
\hline
\end{tabular}




\begin{tabular}{|c|c|c|}
\hline & & $\begin{array}{l}\text { needs, and past experiences of potential adopters" } \\
\text { (Moore \& Benbasat, 1991, p. 195). }\end{array}$ \\
\hline & Results & "the tangibility of the results of using the \\
\hline & Demonstrability & $\begin{array}{l}\text { innovation, including their observability and } \\
\text { communicability" (Moore \& Benbasat, 1991, p. } \\
\text { 203). }\end{array}$ \\
\hline & $\begin{array}{l}\text { Voluntariness } \\
\text { of Use }\end{array}$ & $\begin{array}{l}\text { "the degree to which use of the innovation is } \\
\text { perceived as being voluntary, or of free will" } \\
\text { (Moore \& Benbasat, 1991, p. 195). }\end{array}$ \\
\hline Social Cognitive & Outcome & "the performance-related consequences of the \\
\hline Theory (SCT) & $\begin{array}{l}\text { Expectations - } \\
\text { Performance }\end{array}$ & $\begin{array}{l}\text { behaviour. Specifically, performance expectations } \\
\text { deal with job-related outcomes" (Venkatesh et al., } \\
\text { 2003, p. 432). }\end{array}$ \\
\hline & Outcome & "the personal consequences of the behaviour. \\
\hline & $\begin{array}{l}\text { Expectations- } \\
\text { Personal }\end{array}$ & $\begin{array}{l}\text { Specifically, personal expectation deal with the } \\
\text { individual esteem and sense of accomplishment" } \\
\text { (Venkatesh et al., 2003, p. 432). }\end{array}$ \\
\hline & Self-efficacy & $\begin{array}{l}\text { "Judgment of one's ability to use a technology to } \\
\text { accomplish a particular job or task" (Venkatesh et } \\
\text { al., 2003, p. 432). }\end{array}$ \\
\hline & Affect & $\begin{array}{l}\text { "an individual's liking for a particular behaviour } \\
\text { (e.g. computer use or internet use)" (Venkatesh et } \\
\text { al., 2003, p. 432). }\end{array}$ \\
\hline & Anxiety & $\begin{array}{l}\text { "evoking anxious or emotional reactions when it } \\
\text { comes to performing a behaviour (e.g., using a } \\
\text { computer or using the internet)" (Venkatesh et al., } \\
2003 \text {, p. 432). }\end{array}$ \\
\hline
\end{tabular}

Furthermore, UTAUT model is considered to be a validated model. Several studies have adopted and tested the UTAUT in diverse contexts. For example, Oshlyansky, Cairns, and Thimbleby (2007) aimed to cross-culturally validate the UTAUT among nine different countries, including the United States, United Kingdome, Czech Republic, Greece, India, Malaysia, New Zealand, Saudi Arabia and South Africa. The results suggested that UTAUT Model is sufficiently robust and could be used outside of its original country as well as outside of its original language. Moreover, Tang and Chen (2011) reviewed the evolution of research on information technology acceptance models and made a comparative analysis across four main models including: TAM, TAM2, TAM3, and UTAUT. They found that the UTAUT model can explain (70\%) of the variance in usage intention (see Table 4-3). This is considered to be a significant improvement over other models (Venkatesh et al., 2003). 


\begin{tabular}{cc}
\hline & Explained Variance (Adjusted $\mathbf{R}^{\mathbf{2}}$ ) \\
\cline { 2 - 2 } & Intention to use \\
\hline TAM & $45 \%-61 \%$ \\
TAM2 & $34 \%-52 \%$ \\
TAM3 & $53 \%$ \\
UTAUT & $70 \%$ \\
\hline
\end{tabular}

Previous reviews have highlighted the fact that UTAUT is used within different domains such as telecommunication, education, banking and health (Attuquayefio \& Addo, 2014). In particular, it has been revealed that the UTAUT model is used in research designed to investigate the level of user acceptance when using telehealth applications. Li, Talaei-Khoei, Seale, Ray, and MacIntyre (2013) conducted a systematic literature review that aimed to explore the health care providers' acceptance of using different eHealth applications, in line with the Unified Theory of Acceptance and Use of Technology (UTAUT). They found that 93 papers studied different eHealth applications. Fifty-seven studies were related to Electronic Health Record (EHR)/ Electronic Medical Record (EMR). The second most popular application studied was around Telemedicine/Telehealth (by 7/93 studies). The remaining studies addressed the user acceptance of other eHealth applications such as Anaesthesia Information Management System (AIMS), Electronic Logistics Information System, Intensive Care Information System (ICIS) and e-discharge systems. Furthermore, as mentioned previously, that recent systematic review found that UTAUT is used 9 times within the included telehealth studies (Harst et al., 2019).

With respect to exploring user acceptance of using telehealth, particularly in the field of ASD, Zagzoug (2015) used the UTAUT model to determine the factors that influence the behavioural intention of the parents of children with ASD, of using the web platform for Applied Behaviour Analysis (ABA) intervention training (Zagzoug, 2015). However, to the researcher knowledge, no previous study has used the UTAUT model to explore the acceptance levels of parents/carers of children with ASD, of using the telehealth (NODA system) for ASD diagnostic assessment. Overall, several theories and models sought to describe the user's intention to accept and use new technologies. However, because of the reasons stated previously, the UTAUT model appeared to be the most appropriate model to use to explore parent/carer acceptance 
of using the NODA system for ASD diagnostics assessment. Thus, UTAUT will be used as the theoretical model in Study (3) with some modification. These modifications will be presented in Methodology Chapter (5). The following section will discuss the determinants and moderators of the original UTAUT model.

\subsubsection{Determinants and moderators of UTAUT model}

The UTAUT model includes four constructs as direct determinants of behavioural intention: Performance Expectancy, Effort Expectancy, Social Influence and Facilitating Conditions (see Figure 4-8) (Venkatesh et al., 2003). While, Self-efficacy, Anxiety, and Attitude were hypothesised not to be direct constructs of the intention (Venkatesh et al., 2003). The theory also suggests gender, age, experience, and voluntariness of use as moderators for the four key constructs. All of the constructs and moderators are discussed below. The items that were used for each determinant in estimating UTAUT (Venkatesh et al., 2003) are presented in Table 4-4.

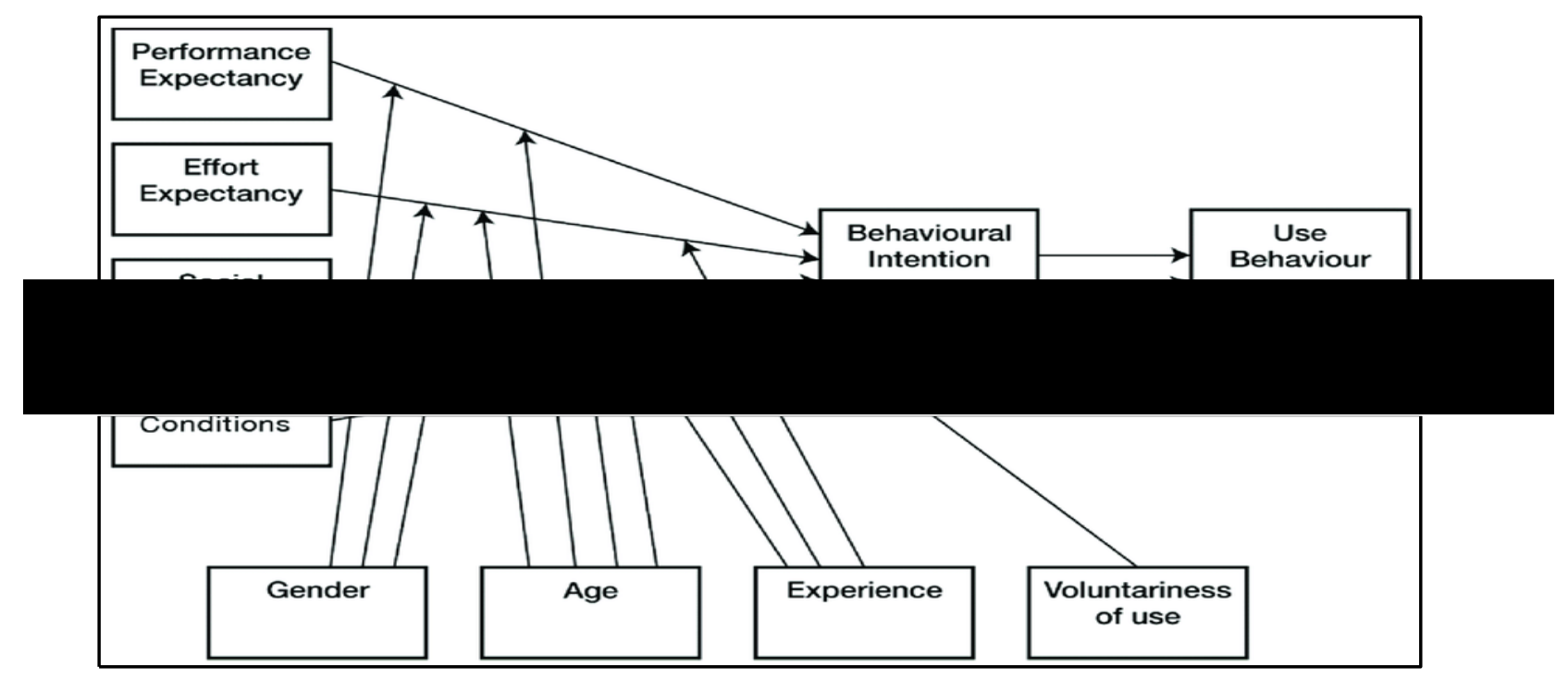

Figure 4-8: Unified Theory of Acceptance and Use of Technology (UTAUT) (Venkatesh et al., 2003) 


\section{Behavioural Intention}

Behavioural intention is identified as a person's readiness to perform a certain behaviour or action (Davis et al., 1989). Consistent with previous models TRA, TPB, TAM, Combined TAM-TPB and MM, behavioural intention has a significant positive influence on technology usage (Venkatesh et al., 2003). Ajzen (1991) suggested that the stronger the intention to perform specific behaviour, the more likelihood of that behaviour being performed (Ajzen, 1991). Behavioural intention might also be used as a dependent variable and independent variable within the studies of technology acceptance models.

\section{Actual Use}

Actual use is the adoption and use of the technology. Many studies revealed that actual use either correlated with behavioural intention or was significantly affected by it (Davis, 1989; Turner, Kitchenham, Brereton, Charters, \& Budgen, 2010; Venkatesh et al., 2003).

\subsection{Constructs as direct determinants of behavioural intention or usage behaviour}

\section{Performance Expectancy}

Venkatesh et al. (2003) defined performance expectancy as the "degree to which an individual believes that using the system will help him or her to attain gains in job performance" (Venkatesh et al., 2003, p. 447). Five constructs from the previous models capture the performance expectancy through: perceived usefulness (TAM/TAM2 and C-TAM-TPB), extrinsic motivation (MM), job-fit (MPCU), relative advantage (IDT), and outcome expectations (SCT) (see Table 4-2) (Venkatesh et al., 2003). They showed that performance expectancy construct was the strongest predictor of intention and was significant in both mandatory and voluntary settings. They expected that performance expectancy is moderated by both age and gender. 


\section{Effort Expectancy}

Venkatesh et al. (2003) defined effort expectancy as the "degree of ease associated with the use of the system" (Venkatesh et al., 2003, p. 450). The three constructs from the other previous models that related to effort expectancy are perceived ease of use (TAM/TAM2), complexity (MPCU) and ease of use (IDT) (see Table 4-2). Effort expectancy construct was significant in both mandatory and voluntary settings (Venkatesh et al., 2003). They suggested that effort expectancy is moderated by age, gender and experience.

\section{Social Influence}

Social influence refers to the "degree to which an individual perceives that important others believe he or she should use the new system" (Venkatesh et al., 2003, p. 451). Social influence captures thoughts represented by the following constructs: subjective norm (TRA, TAM2, TPB/DTPB and C-TAM-TPB), social factors (MPCU), and image (IDT) (see Table 4-2) (Venkatesh et al., 2003). They suggested that social influence construct was non-significant in voluntary settings, whereas it was significant in mandatory settings. They proposed that social influence is moderated by age, gender and experience.

\section{Facilitating Conditions}

Facilitating conditions are defined as the "degree to which an individual believes that an organisational and technical infrastructure exists to support use of the system" (Venkatesh et al., 2003, p. 453). Facilitating conditions construct is embodied as perceived behavioural control in TPB/DTPB and C-TAM-TPB, facilitating conditions in MPCU, and compatibility in IDT (see Table 4-2) (Venkatesh et al., 2003). Facilitating conditions construct becomes non-significant in predicting behavioural intention when performance expectancy and effort expectancy are present. However, they indicated that facilitating conditions will have a significant influence on the actual usage when moderated by experience and age (Venkatesh et al., 2003). 


\title{
4.3.5.1.2 Constructs hypothesised not to be direct determinants of behavioural intention or actual usage
}

\section{Self-Efficacy}

Self-efficacy is defined as “judgment of one's ability to use a technology (e.g. computer) to accomplish a particular job or task" (Venkatesh et al., 2003, p. 432). Venkatesh et al. (2003) found that self-efficacy did not have any impact on behavioural intention. Thus, self-efficacy was dropped from the model.

\begin{abstract}
Anxiety
Anxiety is referring to "evoking anxious or emotional reactions when it comes to performing a behaviour (e.g., using a computer or using the internet)" (Venkatesh et al., 2003, p. 432). Anxiety was initially considered within UTAUT. But, Venkatesh et al. (2003) found that anxiety did not have any effect on the behavioural intention to use the technology, so because of this, it was removed from the final version of UTAUT.
\end{abstract}

\section{Attitude toward using technology}

Attitude toward using technology is identified as "an individual's overall effective reaction to using a system" (Venkatesh et al., 2003, p. 455). Venkatesh et al. 2003 found that attitude toward using technology was a non-significant determinant, hence it was absent from the final model. 


\subsection{Moderators}

\section{Gender}

It was suggested that gender might moderate the influence of performance expectancy, effort expectancy and social Influence on behavioural intention (Venkatesh et al., 2003).

Age

Findings from Venkatesh et al. (2003) suggest that age moderates the effects of performance expectancy, effort expectancy and social influence on behavioural intention.

\section{Experience}

Venkatesh et al. (2003) suggested that limited experience with the system would increase the influence of effort expectancy and social influence on behavioural intention to use the system.

\section{Voluntariness}

Voluntariness is identified as being whether a person has the choice to use the system or not (Rawstorne, Jayasuriya, \& Caputi, 2000). Finding from Venkatesh et al. (2003) suggest that voluntariness moderates the effect of social influence on behavioural intention to use the system. 
Table 4-4: Items used in estimating UTAUT (Venkatesh et al., 2003)

\section{Performance Expectancy}

I. I would find the system useful in my job.

II. Using the system enables me to accomplish tasks more quickly.

III. Using the system increases my productivity.

IV. If I use the system, I will increase my chances of getting a raise.

\section{Effort Expectancy}

I. My interaction with the system would be clear and understandable.

II. It would be easy for me to become skilful at using the system.

III. I would find the system easy to use.

IV. Learning to operate the system is easy for me.

\section{Attitude toward using Technology}

I. Using the system is a bad/good idea.

II. The system makes work more interesting.

III. Working with the system is fun.

IV. I like working with the system.

\section{Social Influence}

I. People who influence my behaviour think that I should use the system.

II. People who are important to me think that I should use the system.

III. The senior management of this business has been helpful in the use of the system.

IV. In general, the organisation has supported the use of the system. 


\section{Facilitating Conditions}

I. I have the resources necessary to use the system.

II. I have the knowledge necessary to use the system.

III. The system is not compatible with other systems I use.

IV. A specific person (or group) is available for assistance with system difficulties.

\section{Self-Efficacy}

I could complete a job or task using the system...

I. If there was no one around to tell me what to do as I go.

II. If I could call someone for help if I got stuck.

III. If I had a lot of time to complete the job for which the software was provided.

IV. If I had just the built-in help facility for assistance.

\section{Anxiety}

I. I feel apprehensive about using the system.

II. It scares me to think that I could lose a lot of information using the system by hitting the wrong key.

III. I hesitate to use the system for fear of making mistakes I cannot correct.

IV. The system is somewhat intimidating to me.

\section{Behavioural Intention to use the System}

I. I intend to use the system in the next $<\mathrm{n}>$ months.

II. I predict I would use the system in the next $<\mathrm{n}>$ months.

III. I plan to use the system next in the $<\mathrm{n}>$ months. 


\subsection{Summary}

The purpose of this chapter was to explore a number of technology acceptance theories and models and then, to describe the theoretical model that will be used to examine the behavioural intention amongst parents/carers of using telehealth for ASD diagnostic assessment, including the reasons for choosing this model from amongst several technology acceptance models. The key theories and models of technology acceptance were discussed including, Theory of Reasoned Action (TRA), Theory of Planned Behaviour (TPB), Diffusion of Innovation Theory (DOI), Technology Acceptance Model (TAM) and Unified Theory of Acceptance and Use of Technology (UTAUT). Many of the technology acceptance theories and models that were discussed have previously been used by other researchers, who attempted to extend and examine them within various contexts. Some of these theories and models were able to explain good part of the variance in the behavioural intention of the users. However, it was decided that the Unified Theory of Acceptance and Use of Technology (UTAUT) should be the base theoretical model for the Study (3). This choice was ascribable to the fact that UTAUT is a global and comprehensive model that combines different founded models and theories explaining technology acceptance. UTAUT has also been tested in many empirical studies and is the subject of multiple extensions. Furthermore, it was found that the UTAUT model can explain $(70 \%)$ of the variance in usage intention, considerably more than other technology acceptance theories and models. Therefore, the UTAUT has a potential to be a useful theoretical model when attempting to understand the behavioural intention of parents/carers of using the NODA system for diagnostic assessment. All of the constructs that were hypothesised in the original UTAUT model as being both direct and indirect determinants of behavioural intention or actual usage were also discussed. The following chapter will discuss the methodology. 


\section{Chapter 5: Methodology of Study (2) and Study (3)}

\subsection{Introduction}

This chapter will present the methodology of this research. The chapter begins by describing the research design. Then, as this research includes three separate but interrelated studies each with different objectives, each study is described separately. The scoping review is the first study and its method is explained briefly as it is described more fully in Chapter 3. The second study relates to exploring the experience of the diagnostic process of ASD in Wales, from the perspective of parents/carers. While, the third study is about exploring the willingness of parents/carers to accept the use of the NODA system for ASD diagnostic assessment. Both Study (2) and Study (3) explain the methods used and data collection process. In Study (3) a hypothesised model development is presented, including the dependent and independent variables. Then the variables and data gathering instrument are presented, and finally the ethical considerations and data analysis.

\subsection{Research design}

It is essential to describe the chosen design for a research, without which there is no clear strategy to either address the research problem or answer the research question. According to Nachmias and Nachmias (1996), research design is considered to be a programme which directs the researcher in the process of collection, analysis and interpretation of observations. The research design is also identified as being the overall approach chosen by the investigator to integrate the varied components of the research in a consistent and rational manner, ensuring that the research problem is effectively addressed (De Vaus \& de Vaus, 2001). The research designs can be classified as quantitative, qualitative, mixed method or multimethod (Creswell, 2014; Morse, 2003). Qualitative and quantitative designs may appear to be contrary methodologies. Qualitative design can be described as a research strategy that places emphasis on the words of individuals or groups rather than quantification through data collection and analysis (Bryman, 2012; Creswell, 2009). Quantitative design is a means of testing theories by examining the association between variables (Cohen, Manion, \& Morrison, 2007; Creswell, 2009). Mixed method design presents a hybrid 
of these two approaches, combining elements of both qualitative and quantitative methodologies within the same research (Creswell, 2009).

However, multimethod design is used when the research involves two or more interrelated studies within an overall topic and aims to solve a major research problem (Morse, 2003). According to Brewer and Hunter (1989), the multimethod approach incorporates any research which plays a part in any way to obtaining a multimethod view of social phenomenon. Multimethod research may be broadly identified as the practice of using two or more methods within the same research, instead of restricting it to the use of one method (Brewer \& Hunter, 1989, 2006). The major difference between multimethod and mixed methods designs is that multimethod design is not limited to combining qualitative and quantitative methods, rather it is open to a diverse range of potential methodological combinations (Hesse-Biber \& Johnson, 2015). Whereas a mixed methods design contains both qualitative and quantitative components that must be combined to create the correct mix of information, data is not usually integrated within studies in multimethod design. Instead each study is designed and conducted to answer a specific sub-question (Anguera, BlancoVillaseñor, Losada, Sánchez-Algarra, \& Onwuegbuzie, 2018; Morse, 2003)

To achieve the main aim of this thesis which is "to examine if telehealth technology can be used (e.g. the degree of acceptance by parents/carers) to increase access to ASD diagnostic services in Wales, UK and reduce the time taken to receive a diagnosis", it was necessary to conduct three different studies, with each study having its own particular objective/objectives. Having considered the specific needs of this research and the differences of various designs, a multimethod approach (see Figure 5-1) was considered to be the most rational choice, dividing this thesis into three studies. Initially, a scoping review was conducted to better understand how telehealth has been used for diagnostic assessment. The intention was then to examine the degree to which parents/carers in Wales accepted the use of telehealth technology (NODA system) for ASD diagnostic assessment. However, as mentioned previously, it became apparent that before examining their levels of acceptance, it was imperative to investigate their overall experience with the ASD diagnostic process in Wales, specifically whether the Refreshed Autistic Spectrum Disorder Strategic Action Plan 2016 (Welsh Government, 2016) had had an impact on whether children were being diagnosed more rapidly and at earlier ages, and if that meant that parents were generally more satisfied 
with the process as a whole. Therefore, three studies were conducted within this piece of research as detailed below:

\section{Study (1): Use of Telehealth for Facilitating the Diagnosis of Autism Spectrum Disorder (ASD): A Scoping Review.}

A scoping review was undertaken in order to meet objective 1, specifically to examine which telehealth approaches were being used in the diagnosis and assessment of autism in both children and adults, whether these are feasible and acceptable, and how they compare with face to face diagnosis and assessment methods (See Chapter 3: Study 1).

\section{Study (2): Exploring Parents/Carers Experience of Receiving a Diagnosis of Autism Spectrum Disorder for their Children in Wales.}

Following the scoping review, a quantitative survey method was used in order to meet objectives 2, 3 and 4 which were to provide an overview of the parents'/carers' journey in obtaining a confirmed diagnosis of ASD for their child in Wales, examine the significant factors associated with the parents/carers degree of satisfaction with the overall ASD diagnostic process in Wales and examine the predictors of their satisfaction with the overall ASD diagnostic process.

\section{Study (3): Exploring Parents/Carers Acceptance of Using Telehealth for ASD Diagnostic Assessment for their Children in Wales.}

In order to meet objective 5, namely to examine the predictors of parents/carers intention to use the telehealth for ASD diagnostic assessment in Wales, a quantitative survey method was used, based on the Unified Theory of Acceptance and Use of Technology (UTAUT) (Venkatesh et al., 2003). The next sections describe the methods used for each study. 
Reserach aim (to examine if telehealth technology can be used (e.g. the degree of acceptance by parents/carers) to increase access to ASD diagnostic services in Wales, UK and reduce the time taken to receive a diagnosis)

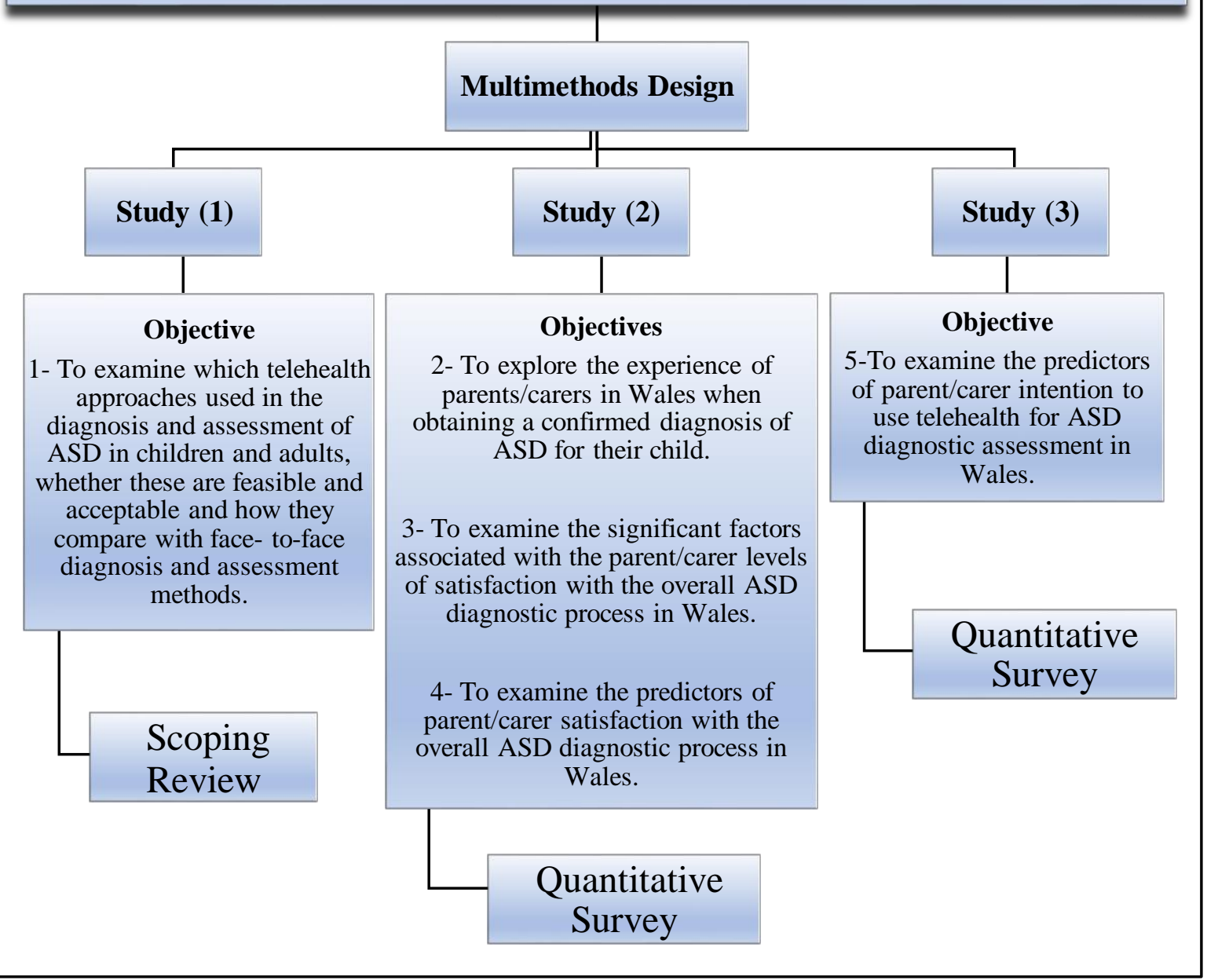

Figure 5-1: Research Design 


\subsection{Study (1): Use of Telehealth for Facilitating the Diagnostic Assessment of Autism Spectrum Disorder (ASD): A Scoping Review.}

See Chapter 3: (Study 1).

\subsection{Study (2): Exploring Parents/Carers Experience of Receiving a Diagnosis of Autism Spectrum Disorder (ASD) for their Children in Wales.}

\subsubsection{Research method}

A quantitative survey design was chosen for this study, in order to explore the experience of parents/carers in Wales with the diagnostic process of ASD. Furthermore, the aim was also to examine the factors associated with their overall satisfaction level with the ASD diagnostic process in Wales, as well as the predictors of their satisfaction. According to Creswell (2014) and Bryman (2012), a survey design provides a quantitative or numerical explanation of the population's trends, views, or attitudes by studying of a statistical sample. The researcher uses sample findings to generalize or draw inferences to the larger population.

It was decided that a quantitative survey research approach for this study was more appropriate than other approaches, as it allows access to a breadth of information that can support in investigating relationships between different variables (Bryman, 2012; Cohen et al., 2007; Creswell, 2009). In addition, such an approach is consistent with other studies that aim to offer an overview of the journey that families experience when seeking to obtain an ASD diagnosis for their children and/or to identify the factors that may be associated with the degree of parent/carer satisfaction with the diagnostic process (Chamak et al., 2011; Crane et al., 2015a; Goin-Kochel et al., 2006; Howlin \& Moore, 1997; Moh \& Magiati, 2012; Siklos \& Kerns, 2007). Thus, a quantitative survey design was used in this study. 


\subsubsection{Population and sample}

The target population of the questionnaire were the parents/carers of children with ASD who live in Wales, UK. The research sample is the segment of the population which is chosen for examination (Bryman, 2012). It was difficult to determine the number of parents/carers of children with ASD in Wales, due to the fact that the UK lacks a register or other accurate record of statistics regarding the probable number of people with ASD (National Autistic Society, 2017a). Furthermore, as time and resources are limited, a nonprobability, convenience sampling technique was used in the current study (Daniel, 2012). Parents/carers were eligible to participate in this study if they: (a) were living in Wales, (b) their child had received a diagnosis of ASD/Autism, (c) and their child was 18 years of age or younger.

\subsubsection{Data collection}

Based on the available time and resources, a cross-sectional approach was used. This meant that data was collected at one point in time (Bryman, 2012). Data was collected in Wales, between around May and July 2018. An online questionnaire was used to gather the data by using online survey software called Survey Monkey. According to Wilson and Mclean (1994), the questionnaire is described as a commonly used useful tool that consists of a series of questions being asked in order to collect information from respondents (Wilson \& McLean, 1994 ). As mentioned earlier, in order to determine the acceptance of telehealth amongst parents/carers (Study 3), it was crucial to initially explore their experiences whilst obtaining a confirmed diagnosis for their child (Study 2). These findings will highlight the existing challenges currently faced by parents/carers throughout the diagnostic process and help in better understanding their attitudes towards the use of telehealth as a means of diagnostic assessment. The questions relating to Study (2) and Study (3) were included together in one questionnaire. So that when the participants finished Study (2) part, they could straight away start on Study (3). This is because it was necessary that the same participants responded to both parts and it was easier for them to fill in both questionnaires at the same time. This therefore allows for simultaneous exploration of the experience those parents/carers had with ASD diagnosis in Wales as well as indicating their acceptance to use NODA as a means of ASD diagnostic assessment. 
Moreover, this research aims to examine the impact of some the main diagnostic process of ASD related variables (Study 1) on whether or not the parent/carer intends to use the NODA system for diagnostic assessment (Study 2). These variables include satisfaction with the overall diagnostic process (it is a dependent variable in Study 1) and the time taken to obtain a diagnosis (it is an independent variable in Study 1). In other words, to understand if parents/carers decisions to either accept or reject the NODA system as a remote diagnostic approach would be influenced by parental satisfaction with the diagnostic process and the time taken to obtain a diagnosis. These variables were therefore added to the proposed model of Study (3) as direct constructs in the UTAUT Model (see Section 5.5.1.2.2, Figure 5-2).

The use of an online questionnaire helps identify a number of benefits (Gillham, 2008; Sarantakos, 2012; Sue \& Ritter, 2012; Walliman, 2006) such as:

- Data could be collected 24 hours a day because the online questionnaire being always open. That meant that the parents/carers of children with ASD were able to complete the questionnaires at a time that best suited their busy lifestyles.

- A link to the online questionnaire can be easily sent to a large number of individuals who may be geographically dispersed.

- Using the online questionnaire can save time and costs, as it is cheap and quick to administer.

- Responses can be stored automatically in a data file. This reduces the amount of time required for manually entering the data for each case.

- The researcher can exert no personal influence when participants complete an online questionnaire.

- Participants have time to consider their responses, which can result in more accurate information being obtained.

- The online questionnaire in this study was not connected to the participants' email addresses therefore guaranteeing them anonymity and enabling them to more truthfully and freely answer the questions. 
The use of the questionnaire has also some disadvantages (Bryman, 2012; Cohen et al., 2007; Walliman, 2006) such as:

- It is not possible to ascertain whether the right person has answered the questionnaire.

- The response rates can be low. If, as is likely, there are differences between those who participate and those who do not, it is possible that the risk of bias in the findings will be greater.

- Simplicity in format and clarity is required in the questions asked, as there is no one present to help respondents if they are having difficulty answering a question.

- There might be a risk that some questions will not be answered. If questions are not answered, this may generate a problem of missing data for the variables that are created

- Not everyone is able to complete questionnaires, particularly the respondents with limited literacy.

\subsubsection{Pre-test (Pilot study)}

To validate the questionnaire instrument, a pilot study was conducted. A pilot study is considered to be a pre-test for the questionnaire, carried out on a small number of individuals of a similar type to those of the proposed sample, before embarking on the actual questionnaire (Walliman, 2006). The purpose of pre-testing is to identify any possible issues in understanding the questionnaire or other problem areas before beginning the research study (Walliman, 2006). A pre-testing study was conducted on 8 parents of children with ASD in Tomms Care (Autism Day Centre) in Port Talbot, Wales. They were asked to complete the questionnaire and comment on the design and format of the questionnaire, the length of the questionnaire, whether the questions were easy to read and understand and any other comments. The entire process was managed by the researcher, who was responsible for providing any explanations raised by any participant, capturing reactions, and administering the duration of the time that needed to fill in the questionnaire. All the participants agreed that the questionnaire was of an appropriate length, format and was easy to read and understand. However, a few 
suggestions were given for alternating phrasing of some questions, to ensure better clarity. All suggestions from the participants were taken into consideration and the necessary modifications were completed in the final version of the questionnaire. The data from pilot study were not included with the study data when the results were analysed as they were not collected using the same standardised methods as the actual study data (Peat, 2002).

\subsubsection{Recruitment process}

In order to reach the parents/carers of children with ASD in Wales, social media (Facebook and Twitter) were used to disseminate the online questionnaire. Facebook was very useful as it has many autism-related groups in Wales, that include parents/carers of children with ASD and autism charities. The admin for each group was contacted via a Facebook message and given full information about the nature of the research, assured that the study had gained approval from the Ethics Committee and that all the participants in the study would be anonymised, provided with the flyer (see Appendix 3) and asked to forward it to their group members. Some administrators allowed the researcher to join the group to introduce herself and post the flyer. The groups that were contacted are shown below with some brief information (see Table

\section{5-1).}


Table 5-1: The Autism-related Groups that were contacted in the Facebook

Facebook group Goal

Number

of

members

Autism Swansea, This group aims to raise awareness of Autism and Asperger

810

Wales \& Beyond Syndrome. It is a place of support where people can chat, exchange information, share details of events, or ask for advice from others.

\begin{tabular}{ll}
\hline MAGIC - Parent & They are an independent parent-led group running social \\
and Carers & groups, activities and events and providing a support \\
Network & network for parents/carers and children with disabilities \\
& across the South Wales area.
\end{tabular}

1000

Life with Autism This group aims to provide support and services to children and their families with a pre/post ASD diagnosis. They are running weekly soft play sessions, hydrotherapy sessions, sensory play, coffee mornings/monthly meetings, fundraising events and training opportunities for parents.

Wales - Act Now It aims to post and share with others news, articles and other 98 For Autism stories of interest about Autism. In addition, to identify the services, provisions and support that have been lost in some areas. This group is also a place for members to chat, share and support each other.

\begin{abstract}
Autism Flintshire This is a group for those who have or care for anyone with Autism. They are providing regular activities such as simple meetings where they can meet others in a relaxed atmosphere, fundraising activities, fun activities and trips to local venues.
\end{abstract} 544

Newport Autism This group includes families, carers and adults with a 400

Support

Group(NAG) diagnosis of ASD or who are on the clinical path to an ASD diagnosis. They meet usually each month at Serennu Children's Centre Newport, where they have talks from professionals, guest speakers, share their experiences, signpost to available resources and offer support to each other.

\begin{tabular}{ll}
\hline Porthcawl & They are a self-funding organisation aiming to raise \\
Aspergers \& & awareness of Asperger Syndrome and Autism. They \\
Autism & encourage group to post articles about ASD and to talk to \\
Community & other group members about their experiences.
\end{tabular}




\begin{tabular}{ll}
\hline Aspergers and & It is identified as a support place where people can chat, \\
Autism in North & $\begin{array}{l}\text { exchange information or ideas. Furthermore, for sharing } \\
\text { Wales Support }\end{array}$ \\
details of events and therapies. \\
Group
\end{tabular}
Autism support A local support group for parents, relatives and carers of for Parents in children with Autism. It aims to support families and provide Cardiff activities.
Autism Act This group is for supporting an Autism Act for Wales. Wales

HAIPAC
It is a support and information service built by parents/carers of children and adults that aims to minimise pressure on parents/carers of individuals living in Carmarthenshire who have additional needs.
The family adviser service is an information service for parent/carers, people with a learning disability, professionals and anyone else with an interest in learning disability and Autism.

\begin{tabular}{ll}
$\begin{array}{l}\text { Carmarthenshire } \\
\text { Family Adviser } \\
\text { Service }\end{array}$ & $\begin{array}{l}\text { The family adviser service is an information service for } \\
\text { parent/carers, people with a learning disability, professionals } \\
\text { and anyone else with an interest in learning disability and } \\
\text { Autism. }\end{array}$ \\
\hline $\begin{array}{l}\text { Friends of heron } \\
\text { heros kids club }\end{array}$ & $\begin{array}{l}\text { They are a newly developed special needs kids club and have } \\
\text { lots of trips and activities throughout the year. }\end{array}$
\end{tabular}

Not mentioned Not mentioned 
The branches also run support, information and social activities for autistic adults, children and their families in their local area such as swimming, athletics and playtime sessions. The following branches of the National Autistic Society that were emailed are:

- NAS Blaenau Gwent Branch

- NAS Bridgend and District Branch

- NAS Caerphilly Branch

- NAS Cardiff and The Vale Branch

- NAS Carmarthenshire Branch

- NAS Cymru Outreach - social group for young people

- NAS Denbighshire and Conwy Branch

- NAS Merthyr Tydfil Branch

- NAS Neath and Port Talbot Branch

- NAS Newport Branch

- NAS Pembrokeshire Branch

- NAS Powys Branch

- NAS Rhondda Cynon Taf Branch

- NAS Swansea Branch

- NAS Wrexham Branch

\subsubsection{Encouraging participation}

Parents/carers of children with ASD are generally very busy people with little time to participate in research projects. Therefore, in order to encourage their participation and improve the survey response rate (Dillman, Smyth, \& Christian, 2014), incentives were used for completing the questionnaires. Use of incentives in this research was approved ethically by the Research Committee in Swansea University Medical School. All completed questionnaires were entered into a prize draw, with $8 £ 25$ Amazon vouchers to be won. If any participant wanted to be entered, he/she had to supply his/her email at the end of the completed questionnaire. This was indicated in the flyer as well as on the welcome page of the survey. All of the winners received online Amazon vouchers via e-mail. 


\subsubsection{Instrument}

The questionnaire began by welcoming the participant and providing general information about the study. Then the Participant Information Sheet (see Appendix 4) and Consent Form (see Appendix 5) were shown. Therefore, the participant was unable to participate without reading the Participant Information Sheet then giving their consent to participate in the study. At the end of the questionnaire, the debriefing was done through the debriefing form (see Appendix 6). They were asked if they would like to receive a copy of the results at the end of the study, and if so to contact the researcher via email. As stated previously, the questionnaire consisted of two studies (see Appendix 7). The sections of Study (2) part are presented below. Study (3) will be presented later in this chapter. Study (2) sections which related to exploring the parent/carer experience of receiving an ASD diagnosis for their children in Wales, were adopted from the studies that conducted by (Centers for Disease Control and Prevention, 2011; Crane et al., 2015a; Daniels et al., 2017; Goin-Kochel et al., 2006; Howlin \& Moore, 1997) (see Table 5-2). 


\subsubsection{Sections of Study (2) part:}

\section{Section (1): Demographic of the respondent (parent/carer)}

This section was designed to capture demographic-related information from respondents. It included questions concerning their gender, age, marital status, highest level of education, where in Wales they lived, their relationship to the child with ASD, the number of overall children and the number of children with ASD.

\section{Section (2): Information about the child with ASD}

Parents/carers were asked to provide some information about their child with ASD including their current age, gender, verbal ability, the age at which parents/carers began to have concerns about their development and the nature of their initial concerns.

\section{Section (3): Diagnostic process}

This section was designed to gather information about the child's age when parents/carers first contacted a health professional and the subsequent role of the health professional (e.g. GP, health visitor, paediatrician, child psychiatrist, psychologist, neurologist, teacher, nurse, or social worker). They were asked to provide information regarding what happened when they contacted a health professional, for example was a diagnosis made, were they referred to another professional, were they sent for tests, were they told that there was "no problem" and not to worry (e.g. "they will grow out of it") or that they should return if things did not improve, the number of professionals that they discussed their concerns with before receiving a diagnosis and the role of these professionals. 


\section{Section (4): Final diagnosis and satisfaction}

Parents/carers were asked to provide information relating to their child's current diagnosis, the role of the person who diagnosed the child, the child's age at the time of diagnosis and the time that elapsed between them first seeking help and finally receiving a diagnosis. Parents/carers were also asked if they received a written report on their child's diagnosis, a follow up appointment with the same professional following the diagnosis, any practical help/ support from health professionals either during the process of seeking a diagnosis or during any follow up appointments after diagnosis.

\section{- Satisfaction with the diagnostic process}

Parents/carers were also asked to indicate their satisfaction with the time taken to obtain a diagnosis, the information they received at the diagnosis and with the overall diagnostic process, by using the Likert scale. Likert scale is identified as one of the common techniques for investigation of participants' attitudes. It is principally a multiple-item measure of a set of attitudes relating to a specific area (Bryman, 2012). Rating scales are commonly used in research and they combine the chance for a flexible response with the ability to determine frequencies, correlations and other types of quantitative analysis (Cohen et al., 2007). A five-point scale is the most used format for indicating level of agreement going from e.g. 'strongly agree' to 'strongly disagree', but also seven-point scale and other formats are used (Bryman, 2012). In this section, 5-point Likert scale including ('very dissatisfied', quite dissatisfied', 'neither satisfied nor dissatisfied', 'quite satisfied', 'very satisfied') was used in order to measure intensity of feelings about the satisfaction with the diagnostic process.

- Stress

Parents/carers were also asked to indicate their level of stress during the diagnostic process by using a 4-point Likert Scale ('not at all stressful', 'not very stressful', 'quite stressful', 'very stressful'). 


\section{Section (5): Post-diagnostic support}

This section was designed to collect information about the type of help/support offered to parents/carers following their child's diagnosis. Furthermore, they were asked to indicate their satisfaction with the help/support that was offered after receiving a diagnosis by using the 5-point Likert Scale ('very dissatisfied', quite dissatisfied', 'Neither satisfied nor dissatisfied', 'quite satisfied', 'very satisfied').

\section{Section (6): Access to services}

The questions relating to access to services were adapted from the National Survey of Children with Special Healthcare Needs (Centers for Disease Control and Prevention, 2011). Parents/carers were asked if they required a referral to see any doctor or receive any service for their child. Those who did require a referral were asked if getting a referral was a major problem, a small problem or no problem at all. Regarding the difficulties using services, they were asked to indicate whether they faced any difficulties or delays in accessing services due to ineligibility, unavailability, waitlists or backlogs, cost and/or lack of information. Moreover, they were asked about their levels of frustration in attempting to secure services for their children; never, sometimes, usually, always, or don't know. 
Table 5-2:Questionnaire Sections (Part 1) and Variables.

\begin{tabular}{|c|c|c|}
\hline Questionnaire sections (Part 1) & Variables & Developed by \\
\hline \multirow{7}{*}{$\begin{array}{l}\text { Section (1): Demographic of the } \\
\text { respondent (parent/carer) }\end{array}$} & Gender & \multirow{4}{*}{ (Crane et al., 2015) } \\
\hline & Age & \\
\hline & Marital status & \\
\hline & Highest level of education & \\
\hline & Relationship to the child with ASD & (Daniels et al., 2017) \\
\hline & Number of overall children & \multirow[b]{2}{*}{$\begin{array}{l}\text { (Howlin \& Moore, 1997) } \\
\quad \text { (Crane et al., 2015) }\end{array}$} \\
\hline & Number of children with ASD & \\
\hline \multirow{5}{*}{$\begin{array}{l}\text { Section (2): Information about the } \\
\text { child with ASD }\end{array}$} & Child's current age & \multirow{2}{*}{ (Crane et al., 2015) } \\
\hline & Gender & \\
\hline & Verbal ability & (Daniels et al., 2017) \\
\hline & Nature of initial concerns & $\begin{array}{c}\text { (Centers for Disease Control and } \\
\text { Prevention, 2011) }\end{array}$ \\
\hline & $\begin{array}{l}\text { The age at which parents/carers began to have } \\
\text { concerns about child development }\end{array}$ & (Crane et al., 2015) \\
\hline
\end{tabular}




\begin{tabular}{|c|c|c|}
\hline \multirow[t]{3}{*}{ Section (3): Diagnostic process } & $\begin{array}{l}\text { Child's age when parents first contacted a health } \\
\text { professional }\end{array}$ & \multirow[b]{2}{*}{ (Crane et al., 2015) } \\
\hline & $\begin{array}{l}\text { What happened when they contacted a health } \\
\text { professional }\end{array}$ & \\
\hline & $\begin{array}{l}\text { Number of professionals that the parents discussed } \\
\text { concerns with before receiving an ASD diagnosis }\end{array}$ & $\begin{array}{c}\text { (Goin-Kochel et al., 2006) } \\
\text { (Mello et al., 2016) }\end{array}$ \\
\hline \multirow{7}{*}{$\begin{array}{l}\text { Section (4): Final diagnosis and } \\
\text { satisfaction }\end{array}$} & Child's age at time of diagnosis & \multirow{3}{*}{ (Crane et al., 2015) } \\
\hline & Child's current diagnosis & \\
\hline & Role of person who diagnosed the child & \\
\hline & $\begin{array}{l}\text { The time that elapsed between them first seeking help } \\
\text { and finally receiving a diagnosis. }\end{array}$ & \multirow{4}{*}{$\begin{array}{l}\text { (Howlin \& Moore, 1997) } \\
\quad \text { (Crane et al., 2015) }\end{array}$} \\
\hline & Receiving a written report on their child's diagnosis & \\
\hline & Follow up appointment with same professional & \\
\hline & Practical help/ support & \\
\hline \multirow{3}{*}{$\begin{array}{l}\text { I. Satisfaction with the } \\
\text { diagnostic process }\end{array}$} & Satisfaction with information received at diagnosis & \multirow{3}{*}{$\begin{array}{l}\text { (Howlin \& Moore, 1997) } \\
\quad \text { (Crane et al., 2015) }\end{array}$} \\
\hline & Satisfaction with time taken to obtain a diagnosis & \\
\hline & Satisfaction with the overall diagnostic process & \\
\hline
\end{tabular}


II. Stress

parents' levels of stress during the diagnostic process

(Crane et al., 2015)

Sort of help/support after their child was diagnosed

Section (5): Post-diagnostic support satisfaction with the help/support that was offered

after receiving a diagnosis

(Crane et al., 2015)

Section (6): Access to services

Difficulty getting referrals

Difficulties or delays in accessing services due to

ineligibility, unavailability, waitlists or backlogs, cost

and/or lack of information.

(Centers for Disease Control and

Prevention, 2011)

Levels of frustration in attempting to secure services

for their children 


\subsubsection{Types of used question}

Closed-format questions were used in the questionnaire of this research. Furthermore, some open-ended questions were used, allowing respondents express their answers in their own words and style (Walliman, 2006). Most of the open-ended questions in this study were "Extension"- "Other (please specify)"- questions. This type of questions was used at the end of the response list, in order to make certain that all choices had been covered (O'Cathain \& Thomas, 2004). For example, these questions related to the marital status of the respondent, their highest level of education, their relationship to the child and the role of the person who diagnosed the child. Some "Substitution" questions were also used to obtain the accurate responses (O'Cathain \& Thomas, 2004). For example, such questions related to the current age of the child, their age when parents first started to have concerns, their age when parents/carers first sought help and at the time of diagnosis.

Moreover, at the end of the access to services section, participants were asked an "Expansion" open-ended question. This is the name given to an open question which is asked after a closed question, to help participants elaborate on the answer they provided for the previous closed question (O'Cathain \& Thomas, 2004). The participants were asked to identify any difficulties/delays that had occurred for any reason other than those mentioned in previous options, "Since [his/her] birth, did you have any difficulties or delays for any other reason?", if they choose the "Yes" option, they were then asked to elaborate on these difficulties. This may have helped them to explain other difficulties that they may have had experienced when accessing autismrelated services for their children in Wales. 


\subsubsection{Data analysis}

After data collection was complete, data was analysed using Statistical Package for Social Sciences (SPSS) software version 25.0. Data analysis included descriptive and inferential statistics being used to answer the research questions. Each piece of raw data was reviewed and checked with the inclusion criteria (Field, 2013). Some cases were removed as they did not complete the questionnaire at all, they were not from Wales, they were still in the diagnostic process and the child had not yet received a diagnosis at the time of the questionnaire or the child was more than 18 years old. The cases with some missing data were included within the sample. Missing data statistics for each variable will be presented in the following chapters. Furthermore, Multiple Imputation (MI) was conducted to impute the missing data, in order to perform the logistic regression analysis with imputed data (Sterne et al., 2009) (more information will be shown below). The following section will present the analysis procedures for Study (2). The data analysis that pertaining to the third study will be shown in the section relating to Study (3).

Descriptive statistics were used to describe participant demographics including means $(M)$ and standard deviations $(S D)$ that were calculated for continuous variables, while frequencies and proportions were calculated for categorical variables. To explore the experience of parents/carers when obtaining a diagnosis of ASD for their children in Wales (objective 2, See 1.6), descriptive statistics were also used. Means and standard deviations were calculated for continuous variables, while frequencies and proportions were calculated for categorical variables.

Whereas, to examine the significant factors that are associated with parents/carers satisfaction with overall diagnostic process in Wales (objective 3, See 1.6), univariate analysis was conducted. All potential determinants as shown below were examined separately in order to explore the characteristics that differentiate between parents/carers who had a negative experience from those who had not a negative experience with the diagnostic process of ASD. Mean value, standard deviation, mean difference and $95 \%$ confidence interval (CI) were calculated for the continuous variables. Frequency, percentage, proportion difference and $95 \%$ confidence interval were also calculated for the categorical variables. Mean difference and proportion 
difference were used instead of Odds Ratio in order to examine the actual value of the difference between the two groups rather than the relative difference.

In respect of the categorical variables, some categories were collapsed as there was a limited number of responses in some categories (Pallant, 2016). For example, the 5Point Likert Scales was used with the satisfaction with the diagnostic process variable. It was coded into a dichotomous variable: negative experience (combining very dissatisfied and quite dissatisfied) and not negative experience (combining very satisfied, quite satisfied, and neither satisfied nor dissatisfied). Having a "neither satisfied nor dissatisfied" option would imply a neutral experience rather than a negative experience with the diagnostic services, thus this group have not been added to the negative experience category. The categories were 'negative experience' and 'not a negative experience' which includes positive and neutral experience of the diagnostic process. 


\section{Variables:}

\section{Demographic variables:}

- Parent/carer age.

- Parent/carer gender.

- Marital status. This was dummy coded to cohabiting/married (combining single with partner, and married) and not cohabiting/married (combining single without partner, separated, divorced, widowed, and living with partner).

- Living area.

- Educational level. It was collapsed into two categories: higher education (Diploma Degree, Bachelor degree, Master degree, and Doctorate Degree) and school level and lower (no qualification, left school at 16 with qualification, and left school at 18 with qualification).

- Number of children.

- Number of children with ASD.

\section{General information about the child related variables:}

- Child's current age.

- Child's gender.

\section{Diagnostic process related variables:}

- Child's age when parents began to have concerns about development.

- Child's age when parents sought professional help.

- Number of professionals consulted before receiving an ASD diagnosis.

- Child's diagnosis.

- Child's age at time of diagnosis.

- Time taken between first seeking help and receiving a diagnosis.

- Satisfaction with information given at diagnosis. This was coded into a dichotomous variable: satisfied (combining very satisfied, quite satisfied, and neither satisfied nor dissatisfied) and dissatisfied (combining very dissatisfied and quite dissatisfied).

- Stress of the diagnostic process. This was coded into a dichotomous variable: stressful (combining very stressful and quite stressful) and not stressful (not 
very stressful and not at all stressful).

- Satisfaction with the post-diagnostic help and support. This was coded into a dichotomous variable: satisfied (combining very satisfied, quite satisfied, and neither satisfied nor dissatisfied) and dissatisfied (combining very dissatisfied and quite dissatisfied).

\section{Access to autism services related variables:}

- Getting referrals. This was dummy coded to being a problem (combining big problem and small problem) and not being a problem (not a problem and don't know).

- Difficulties due to ineligibility for services.

- Difficulties because of issues related to cost.

- Difficulties due to problems related to backlogs or getting appointments.

- Difficulties due to lack of services.

- Difficulties due to lack of information.

A binary logistic regression was conducted to examine predictors of parents/carers satisfaction with the overall diagnostic process of ASD in Wales (Objective 4, See 1.6) within a multivariate model. Logistic regression is identified as a method for modelling a binary response variable's dependence on one or more explanatory variables (Bewick, Cheek, \& Ball, 2005; Field, 2005). Categorical explanatory as well as continuous variables are considered within the logistic regression analysis (Bewick et al., 2005). The binary logistic regression was carried out twice, once with observed data and once with imputed data. Prior to conducting the binary logistic regression, the assumptions of the logistic regression analysis (Pallant, 2016) were tested, including:

1. A dichotomous dependent variable.

The variable satisfaction with the overall diagnostic process was recorded into a dichotomous variable: not a negative experience with overall diagnostic process (combining very satisfied, quite satisfied, and neither satisfied nor dissatisfied) and a negative experience with overall diagnostic process (combining very dissatisfied and quite dissatisfied). 
2. Absence of multicollinearity.

The absence of multicollinearity is the assumption that the predictor variables are not highly correlated. It was assessed using 'Tolerance'. 'Tolerance' is useful tool for diagnosing multicollinearity, which occurred when variables are too closely related. By using SPSS, multicollinearity was checked by looking at 'Tolerance' column in 'collinearity Statistics'. Tolerance values that are very low (less than .1) indicate that the variable has high correlations with other variables (Cohen et al., 2007).

3. Absence of outliers

It is crucial to assess for the presence of outliers. An outlier is identified as a case that varies significantly from the main direction of the data (Field, 2005). The outliers were checked in this study by using 'Casewise List'. The 'Casewise List' is considered as type of statistics that present any outliers. By using the SPSS, the 'Casewise List' was chosen under the logistic regression options and then the 'Casewise List' Table indicated the cases that had to be checked (Pallant, 2016).

\section{- Logistic Regression Analysis with observed data}

Satisfaction with overall diagnostic process was used as a dependent variable and it was dichotomised to a negative experience with the overall diagnostic process and a not negative experience with the overall diagnostic process. Predictor variables that were used in the logistic regression were the same variables as were shown previously. All of the variables were entered in the model. The variable that was the least significant i.e the one with the largest p-value, was removed. Once the variable was removed, it remains excluded. The process is repeated until all remaining variables have a significant p-value (smaller than 0.05) (Bursac, Gauss, Williams, \& Hosmer, 2008; Pallant, 2016). 


\section{- Logistic Regression Analysis with imputed data}

As we stated previously that the data had some missing values. Each piece of raw data including missing data was reviewed and checked. We assumed that the missing values are missing at random (MAR) which means that any difference between the observed values and the missing values might be described by differences in observed data (Sterne et al., 2009). This is maybe because that some parents/carers did not have enough time to complete the questionnaire. Therefore, the missing values of the dependent and independent variables that were included in the previous logistic regression model were imputed by using multiple imputation. Multiple imputation first appeared in the early 1970s and has grown in popularity over the years (Jakobsen, Gluud, Wetterslev, \& Winkel, 2017). It is considered to be a common method to the missing data problem which is available in many statistical packages. It aims to compensate for the ambiguity regarding the missing data by generating many diverse possible imputed data sets and properly incorporating the results attained from each of them (Sterne et al., 2009).

Multiple imputation consisting of three steps (Garson, 2015; Jakobsen, Gluud, Wetterslev, \& Winkel, 2017) was conducted as shown below:

1. Imputation step. An 'imputation' commonly represents one set of plausible values for missing data, while multiple imputation represents several sets of plausible values. By using multiple imputation, missing values for all of the variables (see Page 125-126) were identified and were replaced by a random sample of plausible values imputations (completed datasets). Five imputed datasets have traditionally been suggested to be sufficient on theoretical grounds, but more than 5 datasets (10 datasets) were used to reduce sampling variability from the imputation process (Garson, 2015; Jakobsen, Gluud, Wetterslev, \& Winkel, 2017).

2. Completed-data analysis (estimation) step. The analysis was made separately for each dataset that is produced through the imputation step. Thus, 10 analysis results were constructed.

3. Pooling step. The results obtained from each completed- data analyses were combined into a single multiple- imputation result. 
After the multiple imputation, the logistic regression analysis was conducted for a second time with the imputed data, to examine predictors of parents/carers satisfaction with the overall diagnostic process of ASD. Subsequently, the results of the logistic regression before and after imputation were compared.

\section{Open-ended question}

As we stated previously, participants were asked an "Expansion" open-ended question at the end of the access to services section. Thematic analysis was used to identify and analyse patterns (themes) within open-ended question data (Braun \& Clarke, 2006; Clarke \& Braun, 2013). This method is considered to be flexible and useful, which can possibly provide a rich and detailed explanation of data (Braun \& Clarke, 2006).

To analyse the free text responses, the researcher modified and applied the six thematic analysis approach steps outlined by Braun and Clarke (2006):

- The first step is familiarisation with the data: it includes reading and rereading of the data. While doing this the researcher started marking ideas and taking initial notes.

- The second step is generating initial codes: in this study, this was done manually by using highlighters to identify the interesting aspects in the data items.

- The third step is reviewing themes: This step began when the researcher developed a set of candidate themes. The researcher reviews the defining themes to ensure whether the themes start to define the nature of each individual theme, and the relationship between the themes.

- The fourth step is searching for themes: a theme is a coherent and meaningful pattern in the data relevant to the research question. This step needs re-focusing the analysis at the broader level of themes.

- The fifth step outlined by Braun and Clarke is defining and naming themes: in this step, the researcher identified the 'essence' of each theme and constructed a concise and informative name for each one. 
- The sixth step is writing up: this final step involves writing up the findings of the open-ended question of this study. Representative quotations were also selected from the participants' text responses as illustrative examples.

\subsubsection{Ethical considerations}

Prior to the data collection process, the study received Ethical Approval from the Research Committee in Swansea University Medical School (see Appendix 8). Ethical principles towards participants of the study were considered. Participation in this research was voluntary, and the confidentiality and anonymity of participants were guaranteed.

The flyer included the questionnaire's link. Accessing the link would open up the questionnaire. The questionnaire contained the Participant Information Sheet (see Appendix 4) that provided information about the nature of the study. The participants were informed that their participation was voluntary and that they could withdraw at any time, without having to give a reason. In addition, they were informed that any information collected from them would be anonymous, thereby ensuring their privacy and confidentiality at all time. After that, the Consent Form (see Appendix 5) was shown. They were asked to provide their consent if they would like to participate in the study. They were not allowed to access the questionnaire until they had successfully read the information participant sheet and agreed to participate.

As we stated previously, Survey Monkey was used to collect the data. Once the surveys were completed, the data was downloaded and stored in the researcher's password-protected university based computer as well as supervisors' university based computers, all of which are protected by passwords. In addition, the folders that are contained these files were encrypted and protected by passwords. Consequently privacy, confidentiality, and anonymity were strictly protected. At the end of the questionnaire, the debriefing was done through the debriefing form (see Appendix 6). 
Participants were asked if they would like to receive a copy of the results after participating at the end of the study, in which case they had to contact the researcher via email. All potential subjects were also given the option of contacting the researcher and her supervisors by emails, if they had any questions regarding the study, or would like more information. The data collected from participants will be securely stored until $\mathrm{PhD}$ research has been finalised in an office belonging to one of the supervisors and after that, the data will be destroyed. The next section discusses the methods of the Study (3). 


\subsection{Study (3): Exploring Parents/Carers Acceptance of Using NODA for ASD Diagnostic Assessment for their Children in Wales}

\subsubsection{Developing a Hypothesised Model}

As stated previously in Chapter 4, the Unified Theory of Acceptance and the Use of Technology (UTAUT) Model (see Figure 4-8) (Venkatesh et al., 2003) was selected from several different technology acceptance models, in order to determine the key factors influencing the intention of parents/carers to use the NODA system for ASD diagnostic assessment. The UTAUT Model has helped to generate the questions section that related to parents/carers acceptance of using NODA system for remote diagnostic assessment. Venkatesh et al. (2003) advocates modifications to the UTAUT model in order to improve its predictability when used in other contexts such as healthcare, making it more relevant to any research aim and objectives pertaining to investigating user acceptance and the use of technology. Thus, the UTAUT was adapted so as to fit better into the context of the current study, aiming to enhance the prediction of the acceptance by the parent/carer to use the NODA system for ASD diagnostic assessment. The variables and items that were used in the questionnaire are shown in Table 5-3. The next section explains the dependent and independent variables of the proposed study's theoretical model and the null hypothesis (see Figure 5-2). 


\subsubsection{Dependent variable:}

\section{Behavioural Intention}

The behavioural intention of parents/carers to use the NODA system for ASD diagnostic assessment was used as a dependent variable in this study.

\subsubsection{Independent variables:}

\subsection{Existing direct determinants in UTAUT model:}

\section{Performance Expectancy}

Performance expectancy is described within this study as the degree to which a parent/carer of a child with ASD believes that use of the NODA system (if it had been available before child diagnosis) would help him or her to receive an earlier diagnosis. The performance expectancy concept within the UTAUT model is similar to the perceived usefulness construct in TAM (Davis, 1989; Davis et al., 1989). Performance expectancy is considered to be one of the strongest, if not the strongest predictors of a user's behavioural intention to adopt technology (Davis, 1989; Lin, Wong, \& Tseng, 2016; Rho, Kim, Chung, \& Choi, 2015; Venkatesh \& Davis, 2000; Venkatesh et al., 2003; Zagzoug, 2015). Zagzoug (2015) indicated that performance expectancy affects the behavioural intention of the parents of children with ASD to use telehealth in order to receive online Applied Behavioural Analysis (ABA) training. Furthermore, a study by Choo, Dettman, Dowell, and Cowan (2019) showed that the provision of clear and useful results was considered essential as highlighted by parents to use the language tracking wearable technology for supporting the language development of the child (Choo, Dettman, Dowell, \& Cowan, 2019). It has also been demonstrated that the greater the performance expectancy, it is more likely that mobile health (mHealth) services that supported by using mobile devices would be adopted. For example, Hollier's et al. (2018) revealed that perceived usefulness was an important factor that influenced the behavioural intention of parents to use the proposed guided imagery therapy app for their children (this app was created to treat functional abdominal pain disorders via the remote delivery of recorded audio sessions). There were similar findings around the adoption of mHealth services for self-management amongst 
patients with hypertension (Breil, Kremer, Hennemann, \& Apolinário-Hagen, 2019) and the elderly (Hoque \& Sorwar, 2017; Quaosar, Hoque, \& Bao, 2018). Therefore, this study considered performance expectancy to be a direct determinant in the model that could influence the intention of parents/carers to use the NODA system for ASD diagnostic assessment.

\section{Effort Expectancy}

In this study, effort expectancy is described as the degree of ease associated with the use of the NODA system for diagnostic assessment of ASD. The effort expectancy concept within the UTAUT model is similar to the perceived ease of use construct in TAM (Davis, 1989; Davis et al., 1989). Many studies have shown that perceived ease of use or effort expectancy had a significant influence on intention (Cimperman et al., 2016; Davis, 1989; Hennemann, Beutel, \& Zwerenz, 2016; Hoque \& Sorwar, 2017; Rho et al., 2015; Van Houwelingen, Ettema, Antonietti, \& Kort, 2018). Effort expectancy has been considered as one of the predictors that was statistically significant in explaining the perceived behavioural intention toward healthcare wearable devices (e.g. fall and wandering detection, Clevercare smartwatch, activity monitoring devices) among patients with dementia as reported by caregivers (Dai, Larnyo, Tetteh, Aboagye, \& Musah, 2020). A study by Breil et al. (2019) also found that performance expectancy had a direct impact on the intention of patients with hypertension to adopt mHealth services for self-management (Breil et al., 2019). However, other studies did not find there to be such an influence (Lin et al., 2016; Macdonald, Perrin, Hyett, \& Kingsley, 2019; Zagzoug, 2015). The Zagzoug study (2015) argued that, parents do not seem to be concerned about the complexity of the online intervention, and they may be willing to use a system they felt would provide benefits and make the effort of using the system worthwhile. Therefore, they gave less weight to the effort expectancy. In the context of this study, the author hypothesises that effort expectancy will influence the behavioural intention to use the NODA system for ASD diagnostic assessment. This would allow the researcher to test the influence, in order to find out whether or not there is indeed such an influence. 


\section{Social Influence}

Research into the adoption of technology found that social influence affects the behavioural intention to use the technology (Venkatesh \& Davis, 2000; Venkatesh et al., 2003). In this study, social influence is defined as the degree to which a parent/carer perceives that important others believe he or she should use the NODA system. For instance, if a parent/carer were to think about using the telehealth to obtain a diagnosis for their child, social influence would be what he considers to be the opinions of others (e.g. family members, friends, professionals) about him using the system. Findings within many studies carried out by many scholars suggest the importance of social influence in determining the adoption of technology (Dai et al., 2020; Hennemann et al., 2016; Hoque \& Sorwar, 2017; Kim et al., 2018; Lin et al., 2016; Quaosar et al., 2018; Rho et al., 2015; Zagzoug, 2015). For example, Hennemann et al., (2016) indicated that uptake of a Web-based aftercare system among a different diagnostic sample (paediatric, cardiologic, psychosomatic, and orthopaedic) which could support with the transition to daily life after hospital discharge and stabilise treatment outcomes, increased along with the positive opinions of important others about significance of the system, such as family, friends and general practitioners. A study by Kim et al. (2018) showed that social influence had a significantly positive influence on behavioural intention either to use, or carry on using telehealth, for services related to depression care among functionally dependent older patients. However, other studies (Cimperman et al., 2016; Hoogenbosch et al., 2018) found that social influence had no significant effect on behavioural intention to use the technologies. In the context of this study and consistent with some previous studies, hypothesise that social influence will be influential on the behavioural intention to use the NODA system for diagnostic assessment of ASD. This would allow the researcher to examine if, in this context, social influence would have any significant influence.

\section{Facilitating conditions}

In this study, facilitating conditions are defined as the degree to which a parent/carer believes that sufficient resources (e.g. internet, computer, smartphone, iPad) exists to support use of the NODA system. Findings of a number of studies in the acceptance of technology field have demonstrated that the facilitating conditions construct has a positive influence and is a significant predictor of either the intention to use or actual 
usage (Cimperman et al., 2016; Kim et al., 2018; Lin et al., 2016; Rho et al., 2015; Van der Vaart, Atema, \& Evers, 2016; Venkatesh et al., 2003; Zagzoug, 2015). However, according to the Venkatesh et al. (2003), facilitating conditions become insignificant in predicting behavioural intention when effort expectancy and effort expectancy determinants are present in the same model. Within the context of this study, it is hypothesised that facilitating conditions will have an influence on behavioural intention. This would allow for re-examining of the relationship to see if facilitating conditions have an effect within the context of this study.

\subsection{Added direct determinants in amended UTAUT model:}

\section{Anxiety}

Anxiety is not entirely new to the technology acceptance models as it was originally a part of the Social Cognitive Theory (Bandura, 1989). Anxiety is defined in this study as the emotional or anxious reactions when a parent/carer anticipate an interaction with the NODA system. As we stated previously, anxiety was initially considered within the UTAUT model. But, Venkatesh et al. (2003) found that anxiety did not have any effect on the behavioural intention to use the technology, because of which it was removed from the final version of UTAUT. However, other studies determined that anxiety had a significant impact on the users' behavioural intention to adopt technology services or technology usage (Chang \& Im, 2014; Guo, Sun, Wang, Peng, \& Yan, 2013; Hoque \& Sorwar, 2017; Or \& Karsh, 2009). Therefore, in the context of this study and consistent with previous studies, we added anxiety to the UTAUT model, as a direct determinant that may have an effect on the intention of the parents/carer to use the NODA system for diagnostic assessment.

\section{Self-Efficacy}

Another important dimension is the self-efficacy determinant. Self-efficacy is defined in this study as the belief by the parent/carer that he/she has the ability to use the NODA system. Self-efficacy was removed from the final version of UTAUT, as Venkatesh et al. (2003) found that self-efficacy had no impact on behavioural intention. However, Zagzoug (2015) has recommended that self-efficacy be 
investigated in any future research concerning the prediction of technology acceptance, as it was not examined in their original research. Furthermore, other research found that the self-efficacy factor was a significant predictor of behavioural intention or actual use of technology (Alvseike \& Brønnick, 2012; Macdonald et al., 2019; Van Houwelingen et al., 2018). For example, Alvsekie and Bronnick (2012) revealed that self-efficacy had a significant influence on the acceptance of smart house technology (by using iPad) that aimed to enable older people to manage day-to-day activities independently in their homes for a longer time, suggesting that low selfefficacy decreases the capability of the elderly to use the technology. Macdonald et al. (2019) also showed that self-efficacy had an impact on the intention of people with diabetes to adopt smart insole technology which is considered as form of Information and Communication Technologies (ICT) for diabetes self-management, particularly in the management of foot monitoring, in order to prevent diabetes-related foot disease (DFD) such as ischemia, infection or ulceration. Therefore, the study will explore the possible direct influence between self-efficacy and behavioural intention of parents/carers to use the NODA system for diagnostic assessment.

\section{Demographic related variables}

\section{Gender}

As discussed in Chapter (4), the original UTAUT model considered gender to be a moderator. However, this study considered gender to be one of the direct determinants that may influence behavioural intention in using the NODA system for ASD diagnostic assessment. Although some studies have found that gender had no significant effect on adopting the use of health technology (Albrecht et al., 2017; Breil et al., 2019; Cimperman et al., 2016; Dou et al., 2017; Eichenberg, Wolters, \& Brähler, 2013; Kim et al., 2018; Zagzoug, 2015), others find that such influence does exist (Hennemann et al., 2016; Kontos, Blake, Chou, \& Prestin, 2014). Within the context of this study, it is hypothesised that gender will have an influence on behavioural intention. This would allow for examining the relationship to see if gender has an effect within the context of this study. 
Age

As discussed Chapter (4), the original UTAUT model considered age to be a moderator. However, this study considered age to be one of the direct determinants. Age has been identified by several studies as playing a significant role in the behavioural intention to use technology, as well as the actual usage (Albrecht et al., 2017; Breil et al., 2019; Czaja et al., 2006; Eichenberg et al., 2013; Kontos et al., 2014). Breil et al.'s (2002) findings suggested that older adults with hypertension were less likely than younger adults to accept the use of the mHealth app for self-management. Albrecht et al. (2017) also revealed that age was a significant predictor of intention to use smart devices in a health-related context among patients. This was also supported by Eichenberg et al.'s (2013) study. Thus, in the context of this study, we hypothesise that age will have an influence on the behavioural intention to use the NODA system for the diagnostic assessment of ASD. This would allow the researcher to examine if, within this context, age would have any significant influence.

\section{Level of education}

Although Venkatesh et al., (2003) did not include level of education as a determinant in the UTAUT model, some findings of previous studies (Albrecht et al., 2017; Eichenberg et al., 2013; Hennemann et al., 2016; Zagzoug, 2015) demonstrated that level of education can influence a person's behavioural intention to use technology. Therefore, the study will explore the possible direct influence between level of education and behavioural intention of parents/carers to use the NODA system for diagnostic assessment.

\section{Diagnostic process of ASD related variables}

Study (2) found that the time taken between parents/carers seeking help and receiving diagnosis was a predictor of their satisfaction with the overall diagnostic process. It became apparent that it was important to examine whether the time taken to get a diagnosis, as well as satisfaction with the ASD diagnostic process can influence the intention of parents/carers in Wales to use the NODA for ASD diagnostic assessment. Therefore, they were added to this study as direct constructs to the UTAUT model. 


\section{Technology experience related variables}

Although Venkatesh et al. (2003) did not include any of the technology experience variables in the UTAUT model, research has revealed that computer/internet skills construct was one of the factors to influence both the users' intention to use technology and their actual usage (Berkowsky, Sharit, \& Czaja, 2018; Chang \& Im, 2014; John, 2013; Zagzoug, 2015; Zhang, Yu, Yan, Hu, \& Goureia, 2012). Zagzoug (2015) reported that parents of children with ASD who had more computer/internet skills were more likely to accept the use of telehealth. Similar findings were noted in a study conducted by Zhang et al. (2012) which aimed to understand patients' acceptance of the self-service web application. Moreover, it has been suggested by some previous studies that comfort with using devices such as a laptop computer, tablet computer and mobile phone/smartphone can affect users' willingness to use the technology (Eichenberg et al., 2013; Koivumäki, Ristola, \& Kesti, 2008; Zagzoug, 2015). Therefore, within the context of this study and consistent with previous studies, the computer/internet skills, comfort with using a desktop computer, laptop computer, tablet computer and mobile phone/smartphone were added in the UTAUT model as direct determinants that may have an effect on the parents/carers intention to use the NODA system.

\section{Moderators}

As discussed in Chapter (3), the original UTAUT model considers age, gender, experience, and voluntariness of use to be moderators (Venkatesh et al., 2003). This research study did not use these moderators in the proposed model. Instead, age and gender were used as direct determinants in order to examine whether they affect the behavioural intention of parents/carers to use NODA (see Pages 137-138). That is, to find out whether to take into consideration the age of all possible users (parents/carers) and their gender when develop remote ASD diagnostic approaches. However, experience and voluntariness of use were excluded from the amended model, as they are not relevant to this particular study. The null hypothesis of this study is presented below. 
Null hypothesis: the existing direct determinants in UTAUT model (performance expectancy, effort expectancy, social influence, facilitating conditions), added original variables in estimating UTAUT model (self-efficacy, anxiety), demographic variables (age, gender, educational level), technology experience variables (computer/internet skills, comfort with using a desktop computer, laptop computer, tablet computer and mobile phone/smartphone), diagnostic process related variables (time taken to get diagnosis, satisfaction with diagnostic process) will not have a significant influence on the behavioural intention to use a NODA system for ASD diagnostic assessment among parents/carers of children with ASD in Wales 


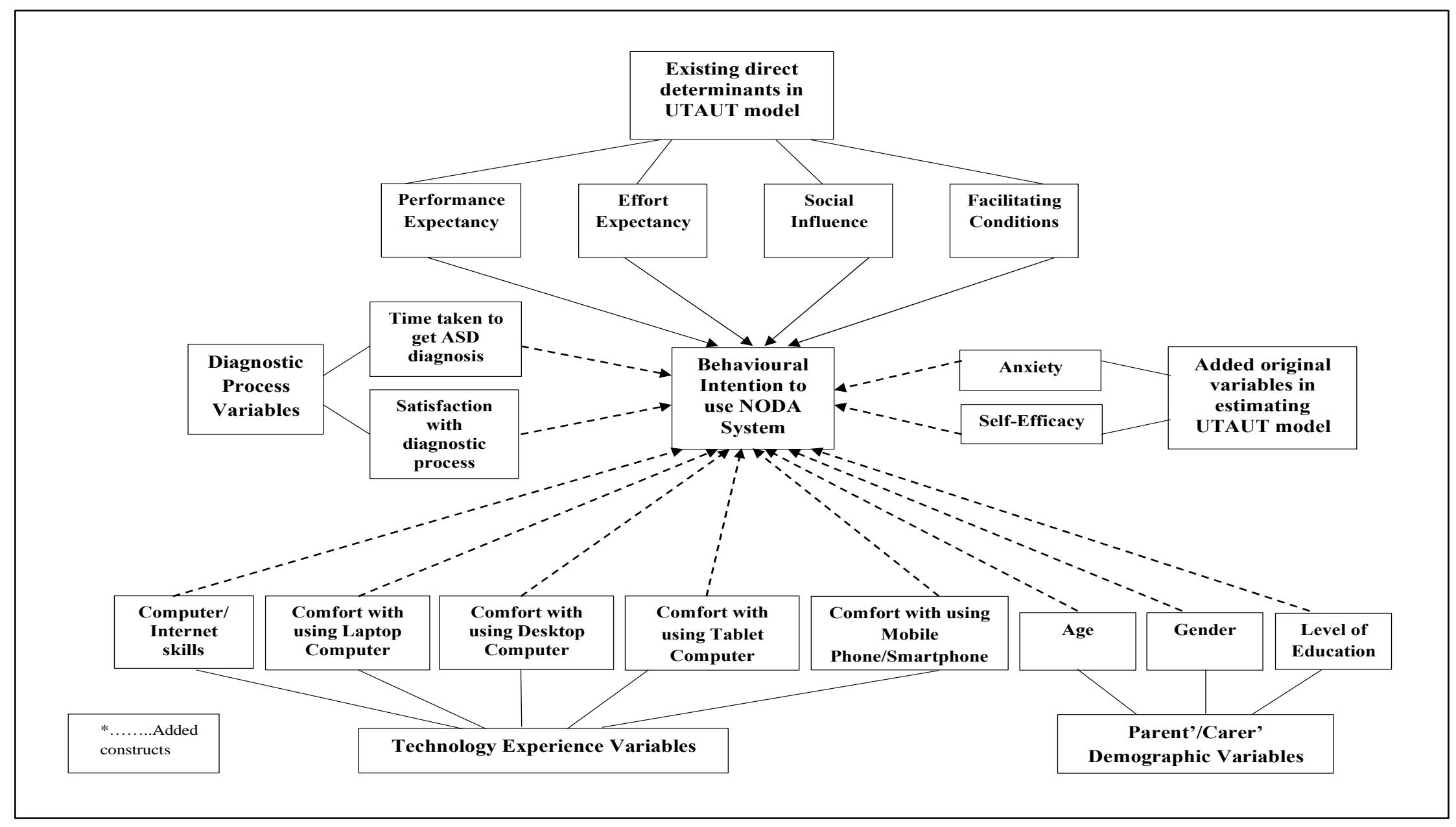

Figure 5-2: Proposed Model of Parents/Carers Behavioural Intention to Use NODA System based on the UTAUT Model 
Table 5-3: The variables and items used in the questionnaire

\begin{tabular}{|c|c|}
\hline Variables & Items/Questions \\
\hline $\begin{array}{l}\text { Behavioural Intention } \\
\text { to use the NODA }\end{array}$ & $\begin{array}{l}\text { If the NODA system had been available before my child's } \\
\text { diagnosis, I would have used the NODA system. }\end{array}$ \\
\hline \multirow{4}{*}{$\begin{array}{l}\text { Performance } \\
\text { Expectancy }\end{array}$} & $\begin{array}{l}\text { I would find the NODA system useful in the diagnosis of my } \\
\text { child. }\end{array}$ \\
\hline & $\begin{array}{l}\text { Using the NODA system would enable me to receive the } \\
\text { diagnosis quickly. }\end{array}$ \\
\hline & $\begin{array}{l}\text { Using the NODA system to obtain a diagnosis, for my child } \\
\text { would be productive in accessing services. }\end{array}$ \\
\hline & $\begin{array}{l}\text { If I use the NODA system, it would increase chances of } \\
\text { getting an earlier diagnosis for my child. }\end{array}$ \\
\hline \multirow{4}{*}{ Effort Expectancy } & $\begin{array}{l}\text { From watching the video, I think I would have clear } \\
\text { understanding of how I would interact with the NODA } \\
\text { system. }\end{array}$ \\
\hline & $\begin{array}{l}\text { If I have training, I think it would be easy for me to become } \\
\text { skillful at using the NODA system. }\end{array}$ \\
\hline & I think I would find the NODA system easy to use. \\
\hline & $\begin{array}{l}\text { I think learning how to use the NODA system would be easy } \\
\text { for me. }\end{array}$ \\
\hline \multirow{3}{*}{ Social Influence } & $\begin{array}{l}\text { My family members would think that I should use the NODA } \\
\text { system. }\end{array}$ \\
\hline & My friends would think that I should use the NODA system. \\
\hline & $\begin{array}{l}\text { I would like to think that professionals would support the use } \\
\text { of the NODA system (e.g. GP, physiologists, health } \\
\text { professionals, teachers). }\end{array}$ \\
\hline \multirow{3}{*}{ Facilitating Conditions } & $\begin{array}{l}\text { I would have the resources necessary to use the NODA system } \\
\text { (e.g. internet, computer, smart phone, iPad). }\end{array}$ \\
\hline & $\begin{array}{l}\text { I would have the knowledge necessary to use the NODA } \\
\text { system. }\end{array}$ \\
\hline & $\begin{array}{l}\text { I think my family and friends would be available for } \\
\text { assistance with NODA system difficulties. }\end{array}$ \\
\hline \multirow{3}{*}{ Self-Efficacy } & $\begin{array}{l}\text { I think I could collect and share video evidence of my child's } \\
\text { behaviour using the NODA system on my own. }\end{array}$ \\
\hline & $\begin{array}{l}\text { I think I could collect and share video evidence of my child's } \\
\text { behaviour using the NODA system if I could call someone for } \\
\text { help if I got stuck (e.g. Help line). }\end{array}$ \\
\hline & $\begin{array}{l}\text { I think I could collect and share video evidence of my child's } \\
\text { behaviour using the NODA system if I had the built-in facility } \\
\text { (e.g. Manual, sample videos and instructions about the system) } \\
\text { for assistance. }\end{array}$ \\
\hline \multirow{4}{*}{ Anxiety } & I would feel apprehensive about using the NODA system. \\
\hline & $\begin{array}{l}\text { It would scare me to think that I could lose a lot of information } \\
\text { using the NODA system by hitting the wrong key. }\end{array}$ \\
\hline & $\begin{array}{l}\text { I would hesitate to use the NODA system for fear of making } \\
\text { mistakes I cannot correct. }\end{array}$ \\
\hline & The NODA System would be somewhat intimidating to me. \\
\hline \multicolumn{2}{|l|}{ Demographic variables } \\
\hline $\mathrm{Ag}$ & ? \\
\hline
\end{tabular}




\begin{tabular}{|c|c|}
\hline Gender & What is your gender? \\
\hline Level of education & What is the highest level of education? \\
\hline \multicolumn{2}{|l|}{$\begin{array}{l}\text { Technology } \\
\text { Experience variables }\end{array}$} \\
\hline Computer/Internet skills & How would you rate your computer/internet skills? \\
\hline $\begin{array}{l}\text { Comfort with using } \\
\text { Desktop Computer }\end{array}$ & How comfortable are you with using Desktop Computer? \\
\hline $\begin{array}{l}\text { Comfort with using } \\
\text { Laptop Computer }\end{array}$ & How comfortable are you with using Laptop Computer? \\
\hline $\begin{array}{l}\text { Comfort with using } \\
\text { Tablet Computer }\end{array}$ & How comfortable are you with using Tablet Computer? \\
\hline $\begin{array}{l}\text { Comfort with using } \\
\text { Mobile } \\
\text { Phone/Smartphone }\end{array}$ & $\begin{array}{l}\text { How comfortable are you with using Mobile } \\
\text { Phone/Smartphone? }\end{array}$ \\
\hline \multicolumn{2}{|l|}{$\begin{array}{l}\text { Diagnostic process of } \\
\text { ASD related variables }\end{array}$} \\
\hline $\begin{array}{l}\text { Time taken to get } \\
\text { diagnosis }\end{array}$ & $\begin{array}{l}\text { How long did it take between first seeking help and receiving } \\
\text { a diagnosis of ASD? }\end{array}$ \\
\hline $\begin{array}{l}\text { Satisfaction with overall } \\
\text { diagnostic process }\end{array}$ & $\begin{array}{l}\text { Overall, how satisfied were you with the diagnostic process as } \\
\text { a whole? }\end{array}$ \\
\hline
\end{tabular}

\subsubsection{Research method}

To investigate the factors that influence acceptance by parents/carers of the NODA system for ASD diagnostic assessment, based on the Unified Theory of Acceptance and Use of Technology (UTAUT), a quantitative survey design was chosen, as it enables access to a breadth of information that can support in testing the theories and investigating relationships between different variables (Bryman, 2012; Cohen et al., 2007; Creswell, 2009). Such a method is consistent with studies in the innovation and technology acceptance field that are aimed towards the understanding of factors influencing user acceptance of particular healthcare technologies (Cimperman et al., 2016; Hennemann et al., 2018; Hoque \& Sorwar, 2017; Jewer, 2018; Kijsanayotin, Pannarunothai, \& Speedie, 2009; Kim et al., 2018; S.-J. Lee, Choi, Rho, Kim, \& Choi, 2018). 


\subsubsection{Population and sample}

As stated previously that, the same population was targeted for both studies (See section 5.4.2).

\subsubsection{Data collection}

See section 5.4.3

\subsubsection{Pre-test Pilot study}

See section 5.4.3.1

\subsubsection{Recruitment process:}

See section 5.4.3.2

\subsubsection{Encouraging Participation}

See section 5.4.4

\subsubsection{Instrument and variables}

To ensure all participants understand the NODA system before proceeding to the second part (Study 3) of the questionnaire, NODA was introduced along with some figurative information about the system, its components as well as its purpose (see Figures 5-3, 5-4, 5-5, 5-6, 5-7) (Nazneen et al., 2016). Furthermore, the participants were required to watch 4 short videos about NODA (each one around one minute) comprising of 4 scenarios (Nazneen et al., 2016) as shown below:

1. Parents concerns scenario https://www.youtube.com/watch?v=nAyw6GOUMlc

2. Family meal time scenario https://www.youtube.com/watch?v=d40_rDAqprg

3. Play with others scenario https://www.youtube.com/watch?v=z3oKRoGOlac

4. Play alone scenario https://www.youtube.com/watch?v=4Clh027Zyo0

The participants were also required to tick a box to show that they had watched the videos, otherwise they were not able to proceed to the questionnaire. 


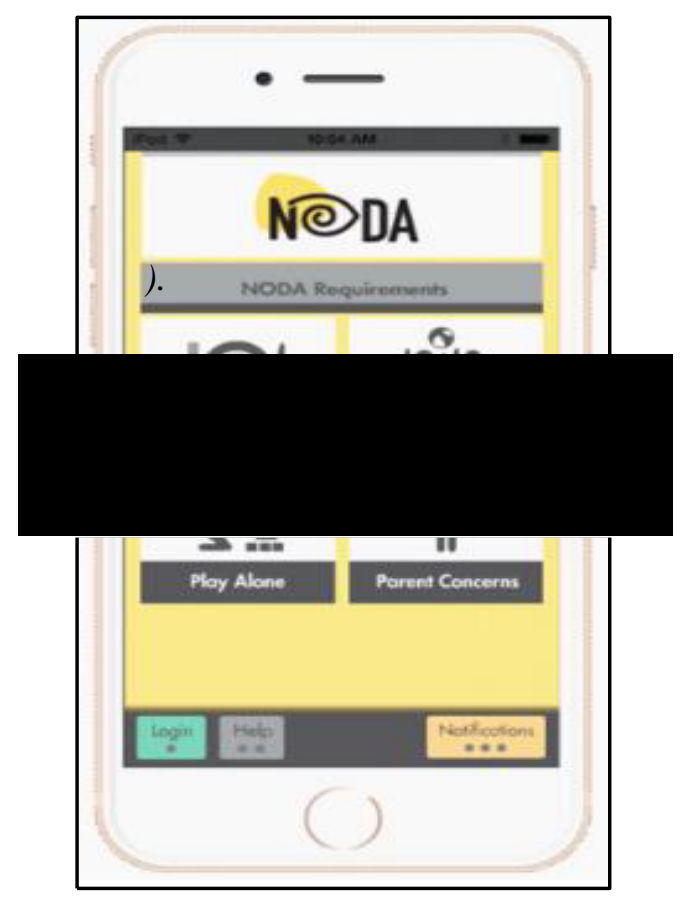

Figure 5-3: NODA

SmartCapture: Home screen showing 4 NODA scenarios (Nazneen et al., 2016)

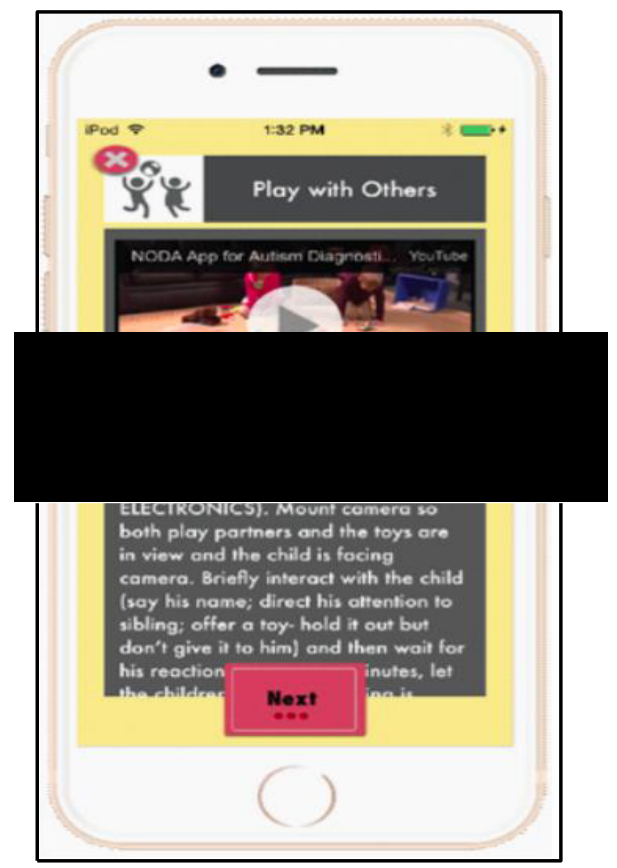

Figure 5-4: NODA SmartCapture: Each scenario includs a 'sample video' and recording instructions about desired interactions with the child during recording (Nazneen et al., 2016)

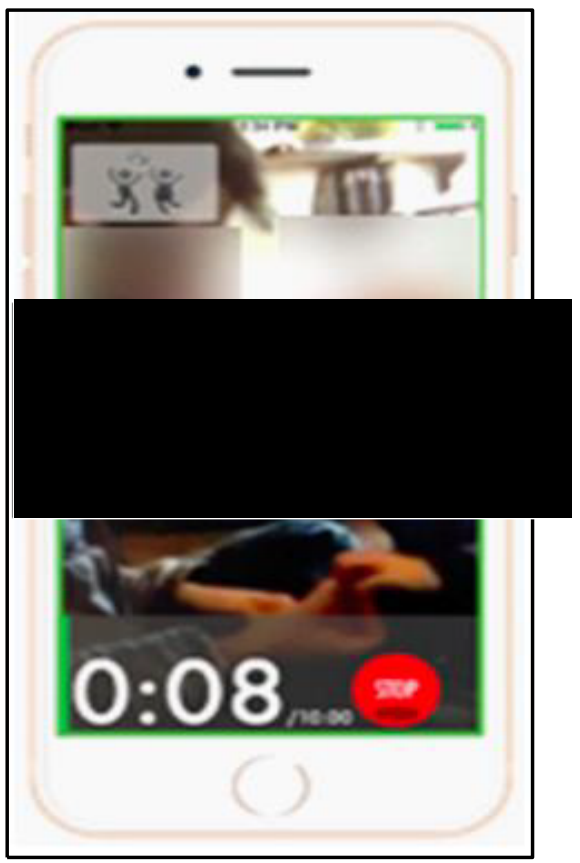

Figure 5-5: NODA SmartCapture: after going throughout instructions, parents can start video recording. Video can be stopped any time or it stops automatically after 10 minutes (Nazneen et al., 2016) 


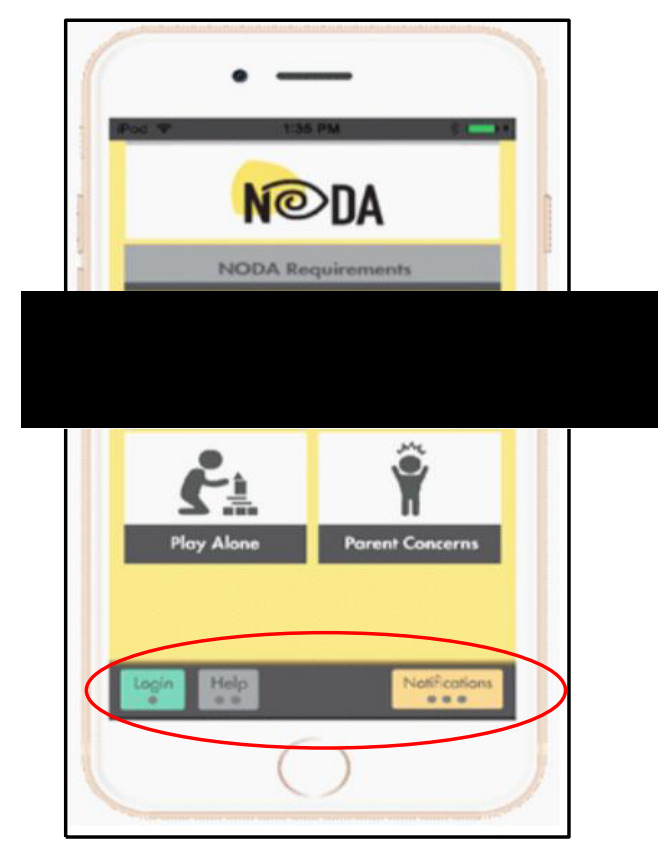

Figure 5-6: NODA

SmartCapture: Home screen shows status of the recorded scenario with options to view, delete or send video to diagnostician. Once video is sent it uploads to NODA

(Nazneen et al., 2016)

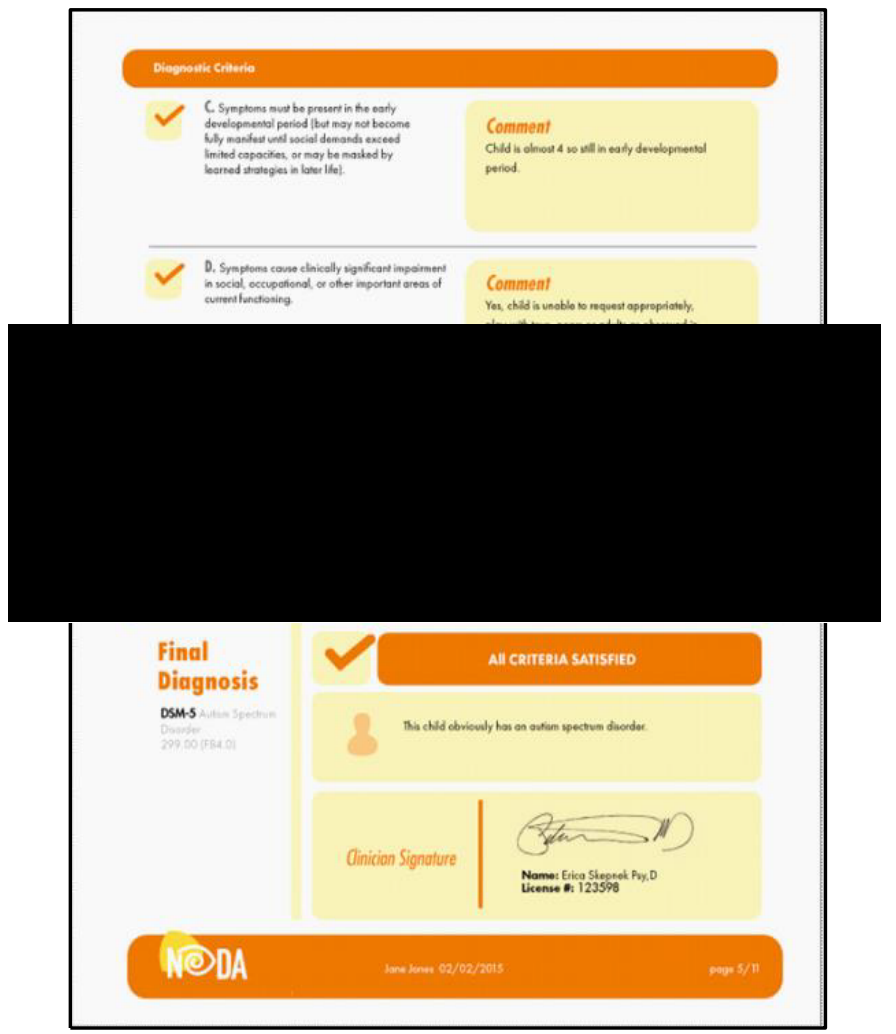

Figure 5-7: NODA Connect: Diagnostic report (Nazneen et al., 2016)

\subsubsection{Sections of Study (3) Part}

\section{Section (1): Technology Experience}

Parents/ carers were asked to rate their computer/internet skills as "novice, basic, average, advanced or expert". Furthermore, they were asked to report how comfortable they were with using a desktop computer, laptop computer, tablet computer and mobile phone/smartphone. These questions were adapted from the Zagzoug (2015) study.

\section{Section (2): Parents/carers Acceptance of using NODA System}

This section was designed to capture the perceptions of parents/carers on using the NODA system for ASD diagnosis. The items (see Table 5-3) were adapted from the those originally used in the estimating UTAUT model (Venkatesh et al., 2003) (see Table 4-4) in Chapter 4. 


\section{Section (3): Provision of NODA system by NHS and Privately}

Parents/carers were asked for their opinion on the use of the NODA system to obtain a diagnosis for their children, if it had been provided free by the NHS as well as being provided privately and accepted by the NHS as an official diagnosis.

\subsubsection{Types of used questions}

Closed-format questions were used in this part of the questionnaire. "Expansion" open-ended question was also used in the second section. This is the name given to an open question which follows a closed question, with the aim of helping participants to elaborate upon their original answer (O'Cathain \& Thomas, 2004). At the end of the section relating to the parents/carers acceptance of use of the NODA system, the participants were asked to indicate their behavioural intention towards using the NODA system, if it had been available prior to their child's diagnosis, with the question "If the NODA system had been available before my child's diagnosis, I would have used the NODA system?". Subsequently, they were asked an "Expansion" open-ended question "Why?", to enable them to explain their reasons behind their degree of acceptance of using the NODA system.

\subsubsection{Data analysis}

After data collection was completed, data were analysed using the Statistical Package for Social Sciences (SPSS) software version 25.0. More information regarding excluded and missing data was previously given in Section 5.4.6.

Initially, descriptive statistics were used to describe participants' experience with technology, including their computer/internet skills and their comfort with using a desktop computer, laptop computer, tablet computer and mobile phone/smartphone. Means and standard deviations were calculated for continuous variables, while frequencies and proportions were calculated for categorical variables. Descriptive statistics were also used to provide an overview of the perceptions of parents/carers about NODA, using UTAUT/added constructs (performance expectancy, effort expectancy, social influence, facilitating conditions, self-efficacy, and anxiety). 
Moreover, descriptive statistics were calculated to provide the expected usage of NODA by parents/carers for diagnostic assessment of their children, if it had been offered previously. Then, to examine the factors that influence the parents/carers intention to use the NODA system for ASD diagnostic assessment in Wales (objective 5, See 1.6), the followings tests were conducted:

\section{Reliability of the constructs (Scale reliability)}

We performed an internal consistency reliability analysis using Cronbach's Alpha for the subscales: performance expectancy, effort expectancy, social influence, facilitating condition, self-efficacy and anxiety. It was evaluated using the guidelines suggested by Hinton, McMurray, \& Brownlow (2004); an Alpha score above .75 is generally considered to show a scale of high reliability, 0.5 to 0.75 is acceptable and taken to indicate a scale of moderate reliability while below this indicates a low reliable scale.

Univariate analysis was conducted. The variables that appear below were examined separately in order to explore the characteristics that differentiate between parents/carers who had a negative intention from those who had not a negative intention to use the NODA system for remote diagnostic assessment. Behavioural intention to use the NODA system was dichotomised to not negative intention (combining strongly agree, agree and don't know) and negative intention (combining disagree, strongly disagree). In respect of the categorical variables, some categories with limited number of responses were collapsed (Pallant, 2016). Mean value, standard deviation, mean difference and 95\% confidence interval (CI) were calculated for the continuous variables. Frequency, percentage, proportion difference and $95 \%$ confidence interval were also calculated for the categorical variables. Mean difference and proportion difference were used instead of Odds Ratio in order to examine the actual value of the difference between the two groups rather than the relative difference. 


\section{Variables}

\section{Existing direct determines in UTAUT model:}

- Performance Expectancy.

- Effort Expectancy.

- Social Influence.

- Facilitating Conditions.

\section{Added original variables in estimating UTAUT model:}

- Self-efficacy.

- Anxiety.

\section{Parent/carer demographics related variables:}

- Age.

- Gender.

- Educational level.

\section{Technology experience variables}

- Computer/Internet skills. It was collapsed into two categories: (novice and basic) and (average, advance and expert).

- Comfort with using a desktop computer, laptop computer, tablet computer, mobile phone/smartphone variables. They were recorded into a dichotomous variable: comfortable (combining very comfortable and comfortable) and not comfortable (combining somewhat uncomfortable, very uncomfortable and natural).

\section{Diagnostic process related variables}

- Satisfaction with the overall diagnostic process of ASD.

- Time taken between first seeking help and receiving a diagnosis.

Following that, a binary logistic regression analysis was conducted to examine the predictors of the intention of parents/carers in Wales to use the NODA system for diagnostic assessment within a multivariate model. Logistic regression is identified as a method for modelling a binary response variable's dependence on one or more explanatory variables (Bewick et al., 2005; Field, 2005). Categorical explanatory as well as continuous variables are considered in the logistic regression analysis (Bewick et al., 2005). The binary logistic regression was carried twice, once with observed data 
and once with imputed data. Before conducting the binary logistic regression, the same previous assumptions of the logistic regression analysis (Pallant, 2016) were tested (see section 5.4.6).

\section{- Logistic Regression Analysis with observed data}

Behavioural intention to use the NODA system was used as a dependent variable and it was dichotomised into not negative intention (combining strongly agree, agree and do not know) and negative intention (combining disagree, strongly disagree). Predictor variables that were used in the logistic regression were the same variables that shown previously. All of the predictors were entered in the model. The variable that is least significant i.e. the one with the largest p-value, was removed. Once the variable is removed, it remains excluded. The process is repeated until all remaining variables have a significant p-value (smaller than 0.05) (Bursac et al., 2008; Pallant, 2016).

\section{- Logistic Regression Analysis with imputed data}

○ (See Page 128-129)

○ For more information about the included variables (See page 149)

\section{Open-ended question:}

As we stated previously, participants were asked an "Expansion" open-ended question at the end of the section relating to the parents/carers acceptance of use of the NODA system. Thematic analysis was used to identify and analyse patterns (themes) within open-ended question data (Braun \& Clarke, 2006; Clarke \& Braun, 2013). This method is considered to be flexible and useful, which can possibly provide a rich and detailed explanation of data (Braun \& Clarke, 2006). To analyse the free text responses, six recursive phases of thematic analysis (Braun \& Clarke, 2006; Clarke \& Braun, 2013) were employed: the researcher becomes familiar with the data, generates first codes, searches for themes, reviews themes, defines and names themes, and writes up. Representative quotations were selected from the participants' text responses as illustrative examples (for more information see Page 129). 


\subsubsection{Ethical consideration}

See section 5.4 .7

\subsection{Summary}

This chapter presents a comprehensive view of the methodology used in this thesis. To achieve the main aim of this thesis, a multimethod approach was utilised, including three studies. The chapter begins by briefly explaining the method of the scoping review (Study 1), as is described in detail in Chapter 3. Then, the quantitative survey method of Study (2) that is related to exploring the experience of parents/carers in Wales around receiving a diagnosis of ASD for their children is discussed. Population and sample, data collection including pilot study and recruitment process, instrument and variables, data analysis and ethical consideration are also explained. Following that, the chapter presents the constructs of the modified theoretical model, the research hypothesis and the quantitative survey method of Study (3) that aimed to examine the predictors of the intention of parents/carers in Wales to use the NODA system for ASD diagnostic assessment. The two following chapters will present the findings of Study (2) and Study (3) (Chapters 6 and 7). 


\section{Chapter 6: Study (2) Results: Exploring the Parents/Carers Experience of Receiving a Diagnosis of Autism Spectrum Disorder for their Children in Wales.}

\subsection{Introduction}

This chapter presents the results of a survey, asking parents/carers to feedback on their experience of the diagnostic process in Wales (Study 2) as outlined in Chapter 5. The chapter begins by presenting descriptive information relating to participant demographics, diagnostic process, final diagnosis and satisfaction, post-diagnostic support and difficulties accessing services. It moves on to present univariate analysis results that show the significant factors associated with parent/carer satisfaction with the overall diagnostic process. The last part of the chapter presents the binary logistic regression model (with missing data and with imputed data) of the predictors of parent/carer satisfaction with the overall diagnostic process. Results from data analysis outlined in this chapter are used to provide answers to the following research questions:

1. What is the parent/carer experience of the ASD diagnostic process in Wales?

2. What are the significant factors that are associated with parent/carer satisfaction with overall diagnostic process?

3. What are the predictors of parent/carer satisfaction with overall diagnostic process?

\subsection{Data Cleaning (Pre-Analysis)}

Responses were obtained from 181 parents/carers (see Figure 6-1). However, 19 were removed as 12 had not completed the questionnaire at all, 2 had children more than 18 years old, 2 were not living in Wales and 3 were still in the diagnostic process and they had not received yet a diagnosis for their children at the time of completing the questionnaire. After removing these cases, the final data consisted of a total of 162 cases. 


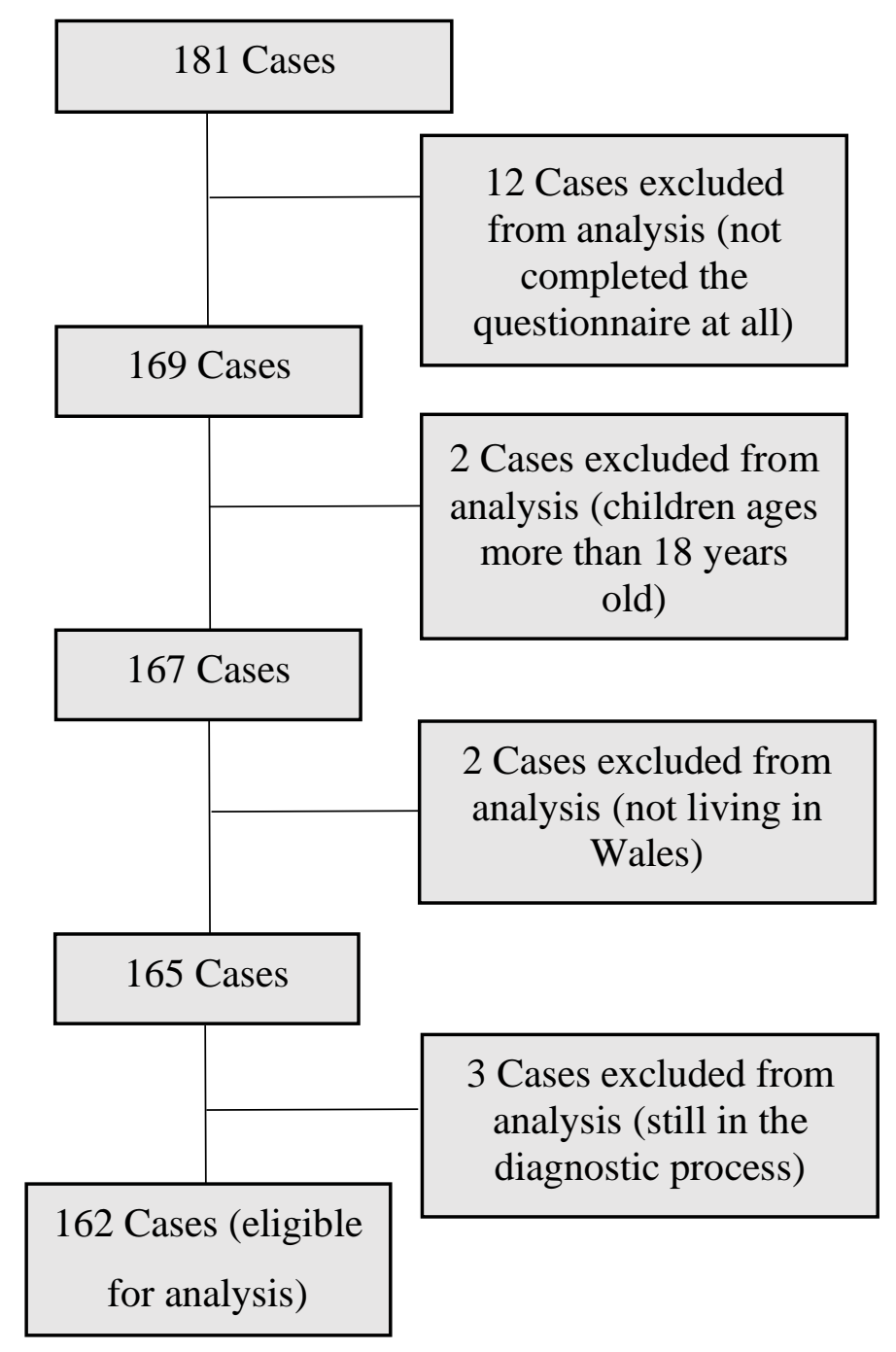

Figure 6-1: Data cleaning

\subsection{Demographics of the parents/carers}

As shown in Table 6-1, the average age of the parents/carers at the time of completing the questionnaire was 38 years (standard deviation $(S D)=8.09$ ). Around 95\% of respondents were female and only $4.9 \%$ were male. Most participants were married (58.6\%), $14.8 \%$ single with partner and $3.7 \%$ separated. In addition, $28.4 \%$ had a Bachelor's degree, $25.9 \%$ left school at 18 with a qualification, $24.1 \%$ left school at 16 with a qualification, 9.3\% had Master's Degree and 2.5\% had Doctorate Degree. The majority of participants were mothers (90.7\%) and only $4.3 \%$ were fathers. Seventy percent of the participants were from South Wales and 29.6\% of them from Mid and North Wales. Forty four percent of the respondents had two children and 
24.2\% had three children. Most of the parents/carers (82.6\%) had only one child with ASD and $15.4 \%$ had two children with ASD.

Table 6-1: Demographics of the parent/carer

\begin{tabular}{|c|c|}
\hline \multicolumn{2}{|l|}{ Gender, n (\%) } \\
\hline Female & $154(95.1 \%)$ \\
\hline Male & $8(4.9 \%)$ \\
\hline Total & $162(100 \%)$ \\
\hline Age (in years), $M(S D),($ Max, Min) & $38.1(8.09),(70,22)$ \\
\hline Total & $160(98.8 \%)$ \\
\hline Missing & $2(1.2 \%)$ \\
\hline \multicolumn{2}{|l|}{ Marital status, n (\%) } \\
\hline Single with partner & $24(14.8 \%)$ \\
\hline Single without partner & $22(13.6 \%)$ \\
\hline Married & $95(58.6 \%)$ \\
\hline Separated & $6(3.7 \%)$ \\
\hline Divorced & $7(4.3 \%)$ \\
\hline Widowed & $3(1.9 \%)$ \\
\hline \multicolumn{2}{|l|}{ Other } \\
\hline Living with partner & $5(3.1 \%)$ \\
\hline Total & $162(100 \%)$ \\
\hline \multicolumn{2}{|l|}{ Education level, n (\%) } \\
\hline No qualification & $9(5.6 \%)$ \\
\hline Left School at 16 with qualification & $39(24.1 \%)$ \\
\hline Left School at 18 with qualification & $42(25.9 \%)$ \\
\hline Bachelor's degree & $46(28.4 \%)$ \\
\hline Master's degree & $15(9.3 \%)$ \\
\hline Doctorate Degree & $4(2.5 \%)$ \\
\hline \multicolumn{2}{|l|}{ Other } \\
\hline Diploma & $7(4.3 \%)$ \\
\hline Total & $162(100 \%)$ \\
\hline \multicolumn{2}{|l|}{ Living area, $\mathbf{n}(\%)$} \\
\hline South Wales & $114(70.4 \%)$ \\
\hline Mid Wales & $24(14.8 \%)$ \\
\hline North Wales & $24(14.8 \%)$ \\
\hline Total & $162(100 \%)$ \\
\hline \multicolumn{2}{|l|}{ Relationship to the child, n (\%) } \\
\hline Mother & $147(90.7 \%)$ \\
\hline Father & $7(4.3 \%)$ \\
\hline Grandparent & $1(0.6 \%)$ \\
\hline Other & $7(4.3 \%)$ \\
\hline Carer & $5(3.1 \%)$ \\
\hline Sister & $2(1.2 \%)$ \\
\hline Total & $162(100 \%)$ \\
\hline \multicolumn{2}{|l|}{ Number of children, $n(\%)$} \\
\hline 1 & $28(18.8 \%)$ \\
\hline 2 & $66(44.3 \%)$ \\
\hline
\end{tabular}




\begin{tabular}{ll}
3 & $36(24.2 \%)$ \\
4 & $12(8.1 \%)$ \\
5 & $6(4.0 \%)$ \\
$6+$ & $1(0.7 \%)$ \\
Total & $149(92.0 \%)$ \\
Missing & $13(8.0 \%)$ \\
\hline Number of children with ASD, n (\%) & \\
1 & $123(82.6 \%)$ \\
2 & $23(15.4 \%)$ \\
3 & $3(2.0 \%)$ \\
Total & $149(92.0 \%)$ \\
Missing & $13(8.0 \%)$ \\
\hline
\end{tabular}

\subsection{General information about the child with ASD}

Characteristics of the child with ASD are presented in Table 6-2. The parents/carers have been asked in the questionnaire to provide the information of the youngest child if they have more than one child with ASD. The average age of the children was 9.19 years ( $S D=4.17$ years) and ranged from 2 years to 18 years. The gender percentage of the children $($ Male $=75.2 \%$, Female $=24.8 \%$ ) was in line with higher numbers of males than females being diagnosed with ASD. The verbal ability of the children ranged from $11.1 \%(\mathrm{n}=17)$ of respondents stating that their child does not talk, to $62.7 \%(\mathrm{n}=96)$ stating that the child uses complex sentences (e.g. "I am tired, and I want to sleep"). Around $38 \%$ of the parents/carers began to have concerns about their child's development when they were between 13-24 months and 29.4\% when they were between 0-12 months. Across the sample as a whole, the most recognised first concerns included behavioural difficulties such as sleeping or eating problems, high activity level, wandering, tantrums, aggressive or destructive behaviour (69.1\%), difficulty playing or interacting with others or a tendency to play alone "in her/her own world" (68.5\%), insisting on maintaining sameness or experiencing difficulties with change (66.7\%), difficulty learning new skills such as toilet training or getting dressed (55.6\%), and an equal percentage of respondents noticed unusual gestures or movements such as hand-flapping, toe-walking, or self- spinning as well as an apparent inability to understand nonverbal communication, such as the tone of voice, facial expressions or other body language cues (52.5\%). 
Table 6-2: General information about the child with ASD

\begin{tabular}{ll}
\hline $\begin{array}{l}\text { Child's age (in years), } \boldsymbol{M}(\boldsymbol{S D}),(\mathbf{M a x}, \\
\text { Min) }\end{array}$ & $9.19(4.17),(18,2)$ \\
Total & $\begin{array}{l}153(94.4 \%) \\
9(5.6 \%)\end{array}$ \\
Missing & $115(75.2 \%)$ \\
\hline $\begin{array}{l}\text { Child's gender, n (\%) } \\
\text { Male }\end{array}$ & $38(24.8 \%)$ \\
Female & $153(94.4 \%)$ \\
Total & $9(5.6 \%)$ \\
Missing & $17(11.1 \%)$ \\
\hline Child's verbal ability, n (\%) & $6(3.9)$ \\
Does not talk & $7(4.6)$ \\
Uses single words only (e.g. "Bubbles") & \\
Uses two- or three-word phrases (e.g. & $27(17.6)$ \\
"Daddy shoe") & \\
$\begin{array}{l}\text { Uses sentences with four or more words } \\
\text { (e.g. "I want more juice") }\end{array}$ & $96(62.7)$ \\
Uses complex sentences (e.g. "I am tired & \\
and I want to sleep") & $153(94.4 \%)$ \\
Total & $9(5.6 \%)$ \\
Missing
\end{tabular}

Child's age when parents began to have concerns about his/her development, $n$

(\%)

0-12 Months

$45(29.4 \%)$

13-24 Months

$58(37.9 \%)$

25-36 Months

$25(16.3 \%)$

37-48 Months

$5(3.3 \%)$

49-60 Months

$6(3.9 \%)$

61-72 Months

$4(2.6 \%)$

73-84 Months

$2(1.3 \%)$

85-96 Months

$5(3.3 \%)$

109-120 Months

$2(1.3 \%)$

133-144 Months

$1(0.7 \%)$

Total

$153(94.4 \%)$

Missing

$9(5.6 \%)$

Parent's/carer's first concerns, n (\%)

Had medical problems such as seizures,

lack of physical growth, or stomach

$19(11.7 \%)$

problems

Didn't make eye contact when talking or

$76(46.9 \%)$

playing with others

Didn't respond when called or didn't

$67(41.4 \%)$

respond to sound

Didn't seem to understand nonverbal

$85(52.5 \%)$

communication, such as understanding what you meant by the tone of voice you used or your facial expressions or other body language cues 
Had behavioural difficulties such as

$112(69.1 \%)$

sleeping or eating problems, high activity

level, wandering, tantrums, aggressive or

destructive behaviour

Had problems with coordination or gross

motor skills such walking

Was not talking at all

$66(40.7 \%)$

$32(19.8 \%)$

Did not talk as well as other children that

$55(34.0 \%)$

were the same age

Some speech skills that he/she had already

$36(22.2 \%)$

developed were lost

Didn't seem to understand what you or

$59(36.4 \%)$

other adults said to him/her

Had problems with fine motor skills such

$73(45.1 \%)$

as using scissors or drawing with crayons

Had difficulty playing or interacting with

$111(68.5 \%)$

others, or played alone "in her/her own

world"

Insisted on sameness or had difficulties

with change

$108(66.7 \%)$

Had difficulty learning new skills such as

$90(55.6 \%)$

toilet training or getting dressed

Had difficulty learning new things such as

$45(27.8 \%)$

the alphabet or numbers

Had unusual gestures or movements such

$85(52.5 \%)$

as hand-flapping, toe-walking, or self-

spinning

Total

$162(100 \%)$

\subsection{The diagnostic process}

Of the 144 participants, $38.9 \%$ ( $n=56)$ first sought help when their child was between 13-24 months and 21.5\% $(n=31)$ when they were between 25-36 months. Different professionals were seen at this time, most commonly a health visitor $(51.4 \%)$ (see Figure 6-2). In the first encounter, around half of the respondents were referred to other professionals (58.3\%) and $22.2 \%$ were told there was "no problem" or told not to worry (e.g., "they'll grow out of it") (see Table 6-3). While, 8.3\% reported other responses such as "Some told to return, later referred to Child and Adolescent Mental Health Services (CAMHS) but could not get appointment till get private diagnosis when suicidal." (Mother of an 18-year- girl, diagnosed with ASD at the age of 17 years), "Told that school had to deal with referral." (Mother of a 17-year- old boy, diagnosed with ASD at the age of 14 years), "Keep under observation, back in 6 
months. If I mentioned ASD I was dismissed. If I did not professional mentioned ASD." (Mother of a 11-year- old boy, diagnosed with Autism at the age of 8 years).

Around $49 \%(\mathrm{n}=70)$ of the parents/carers reported that they discussed their concerns with five or more professionals before receiving an ASD diagnosis for their child, the paediatrician was the professional most often seen by the parents/carers (79.2\%). They saw also the health visitor (68.8\%) and GP (53.5\%) (see Table 6-3). Some parents reported that they had seen other professionals (38.9\%) such as a speech and language therapist, occupational therapist and educational psychologist.

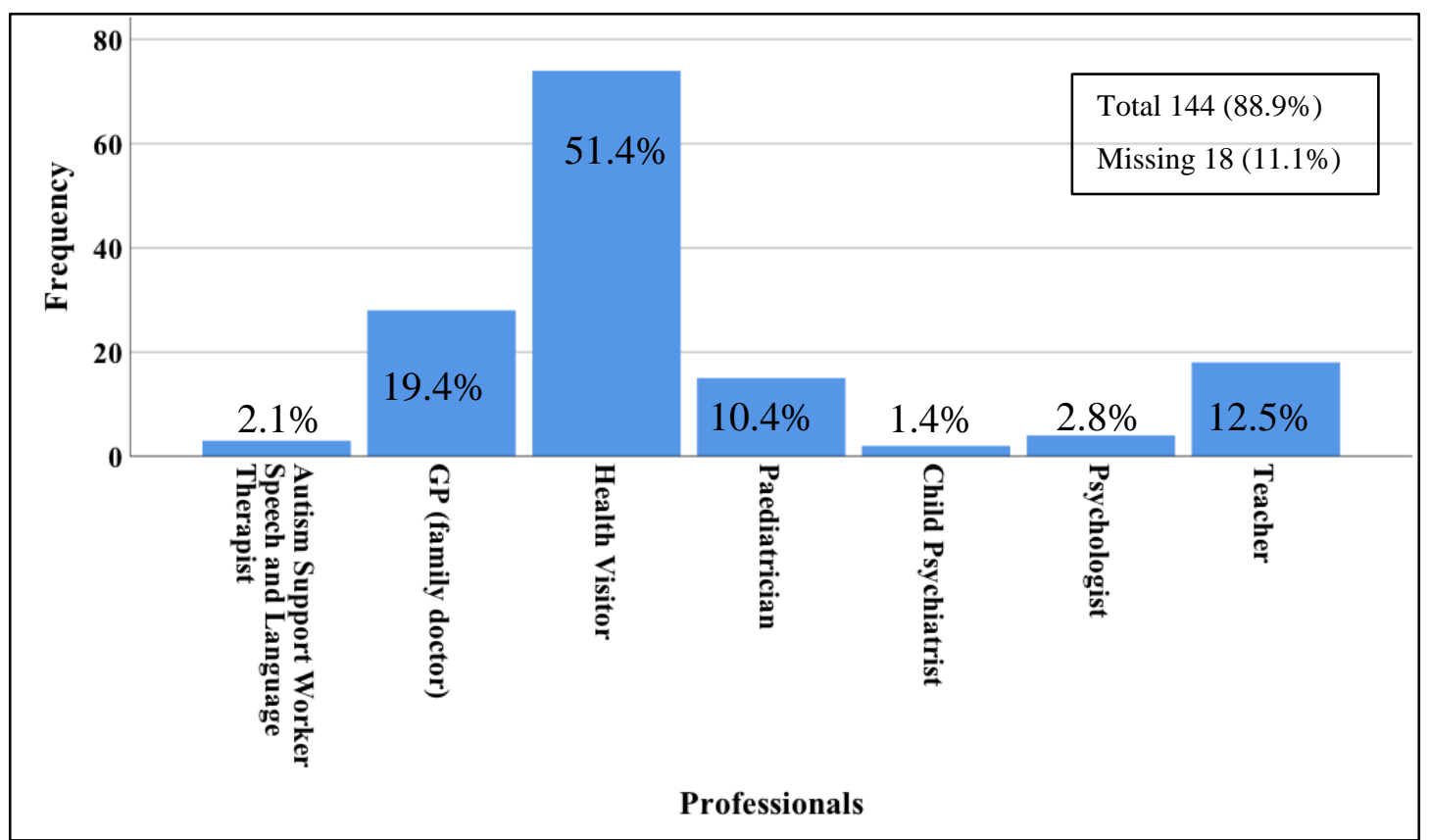

Figure 6-2: Professional who had been seen by parent/carer at the first time 


\begin{tabular}{|c|c|}
\hline \multicolumn{2}{|l|}{$\begin{array}{l}\text { Child's age when parent/carer first } \\
\text { sought help, } n(\%)\end{array}$} \\
\hline $0-12$ Months & $18(12.5 \%)$ \\
\hline 13-24 Months & $56(38.9 \%)$ \\
\hline 25-36 Months & $31(21.5 \%)$ \\
\hline 37-48 Months & $11(7.6 \%)$ \\
\hline 49-60 Months & $8(5.6 \%)$ \\
\hline 61-72 Months & $4(2.8 \%)$ \\
\hline 73-84 Months & $4(2.8 \%)$ \\
\hline 85-96 Months & $4(2.8 \%)$ \\
\hline 97-108 Months & $3(2.1 \%)$ \\
\hline 109-120 Months & $2(1.4 \%)$ \\
\hline $121-132$ months & $1(0.7 \%)$ \\
\hline 133-144 Months & $2(1.4 \%)$ \\
\hline Total & $144(88.9 \%)$ \\
\hline Missing & $18(11.1 \%)$ \\
\hline \multicolumn{2}{|l|}{ What happened in the first } \\
\hline \multicolumn{2}{|l|}{ Diagnosis made } \\
\hline Referred to other professional & $84(58.3 \%)$ \\
\hline Sent for tests & $7(4.9 \%)$ \\
\hline $\begin{array}{l}\text { Told "no problem" or told not to worry } \\
\text { (e.g. " "they'll grow out of it") }\end{array}$ & $32(22.2 \%)$ \\
\hline $\begin{array}{l}\text { Told to return if problems did not } \\
\text { improve }\end{array}$ & $6(4.2 \%)$ \\
\hline Other & $12(8.3 \%)$ \\
\hline Total & $144(88.9 \%)$ \\
\hline Missing & $18(11.1 \%)$ \\
\hline \multicolumn{2}{|l|}{$\begin{array}{l}\text { Number of professionals did } \\
\text { parent/carer discuss concerns with } \\
\text { before receiving an ASD diagnosis for } \\
\text { your child, n (\%) }\end{array}$} \\
\hline 1 & $5(3.5 \%)$ \\
\hline 2 & $15(10.4 \%)$ \\
\hline 3 & $25(17.4 \%)$ \\
\hline 4 & $22(15.3 \%)$ \\
\hline 5 and more & $70(48.6 \%)$ \\
\hline Unsure or don't know & $7(4.9 \%)$ \\
\hline Total & $144(88.9 \%)$ \\
\hline Missing & $18(11.1 \%)$ \\
\hline \multicolumn{2}{|l|}{ Role of the professional(s), n (\%) } \\
\hline GP (family doctor) & $77(53.5 \%)$ \\
\hline Health Visitor & $99(68.8 \%)$ \\
\hline Paediatrician & $114(79.2 \%)$ \\
\hline Psychiatrist & $20(13.9 \%)$ \\
\hline Psychologist & $73(50.7 \%)$ \\
\hline Neurologist & $19(13.2 \%)$ \\
\hline
\end{tabular}


Teacher

$79(54.9 \%)$

Nurse

21(14.6\%)

Social worker

Unsure or don't know

Other

Total

Missing
$17(11.8 \%)$

$5(3.5 \%)$

$56(38.9 \%)$

$144(88.9 \%)$

$18(11.1 \%)$

\subsection{Final diagnosis and satisfaction}

Parents/carers reported a range of diagnoses that included Autism Spectrum Disorder (47.7\%), Autism (37.7\%) and Asperger syndrome (13.8\%) (see Table 6-4). The diagnosis was generally assigned by a multidisciplinary team $(31.6 \%)$, paediatrician (29.3\%), clinical psychologist (12.8\%), psychiatrist (7.5\%) and other diagnosing professionals, included neurologists, speech and language therapists, occupational therapists and educational psychologists, although some parents/carers were unsure about the job title of the final diagnosing professional. The average child's age at time of diagnosis was 6.93 years $(S D=4.096)$, and ranged from 1 years to 18 years. The average delay between the parent/carer initially seeking help and the child receiving a diagnosis of ASD was 46.27 months $(S D=39.96)$.

The majority of those responded received a written report on their child's diagnosis $(82.0 \%)$, however, only $42.1 \%$ received a follow-up appointment with the same professionals following the diagnosis. Moreover, only $16.5 \%$ of parents/carers received a direct offer of practical help/support by health professionals during the process of seeking a diagnosis, 30.8\% were signposted to help, but $44.4 \%$ of parents/carers reported that they had not been offered any help or support during the diagnostic process. Some parents/carers reported other responses such as

"A sheet of paper with some phone numbers."

(Mother of a 13-year- old boy, diagnosed with Autism at the age of 4 years)

"At times yes and at times no. We were refused an assessment by the paediatric neurologist at age 9 or so, and then I had to fight very hard to get a second opinion, which I did when the neurologist retired. Then a five-minute meeting produced an official ASD diagnosis (it had been 'autistic characteristics' with 
a bunch of other stuff up until that point)."

(Mother of a 15-year- old boy, diagnosed with Autism at the age of 14 years)

"Referred to Integrated Autism Service."

(Mother of a 17-year- old boy, diagnosed at the age of 17years)

"Was given a badly photocopied sheet of paper, which contained many out of date contact numbers and told that we were dismissed from CAMHS and we could be re-referred in the future if we needed it."

(Mother of a 12-year- old boy, diagnosed with Autism at the age of 11 years)

Furthermore, only $13.5 \%$ of the parents/carers received a direct offer of practical help/support by health professionals in follow-up appointments after diagnosis and $32.3 \%$ were signposted to help. However, $46.6 \%$ of the parents/carers had not been offered any help or support in follow-up appointments after diagnosis. Some parents provided other responses such as "Just early bird." (Mother of a 11-year- old boy, diagnosed with Autism at the age of 8 years), "Support from educational specialists rather than health." (Mother of a 15-year- old boy, diagnosed with Asperger syndrome at the age of 9 years).

Satisfaction with the diagnosis was rated on a series of 5-point Likert Scale; very dissatisfied, quite dissatisfied, neither satisfied nor dissatisfied, quite satisfied and very satisfied. The majority of parents/carers reported that they were dissatisfied with the time taken to get diagnosis (63.2\%) and only 20.3\% were satisfied (see Figure 6-3). Moreover, $40.6 \%$ of the participants were dissatisfied with the information given at diagnosis and 33.1\% were satisfied (see Table 6-4). When the participants were asked about their satisfaction with the diagnostic process as a whole, the majority were dissatisfied (59.4\%) and only 26.3\% were satisfied (see Figure 6-4). Parents/carers also rated the stress of the diagnostic process on a 4-point Likert Scale; very stressful, quite stressful, not very stressful and not at all stressful. The majority of those who responded to this item felt that the process was very stressful $(68.4 \%)$, while $21.8 \%$ found it quite stressful, $7.5 \%$ found it not very stressful and only $2.3 \%$ found it not at all stressful. 
Table 6-4: Final diagnosis and satisfaction

\begin{tabular}{|c|c|}
\hline \multicolumn{2}{|l|}{ Child's current diagnosis, n (\%) } \\
\hline Autism & $49(37.7 \%)$ \\
\hline Asperger syndrome & $18(13.8 \%)$ \\
\hline Autism Spectrum Disorder & $62(47.7 \%)$ \\
\hline Pervasive Developmental Disorder: Not & $1(0.8 \%)$ \\
\hline Otherwise Specified & \\
\hline Total & $130(80.2 \%)$ \\
\hline Missing & $32(19.8 \%)$ \\
\hline \multicolumn{2}{|l|}{$\begin{array}{l}\text { Role of the person who diagnosed the } \\
\text { child, } n(\%)\end{array}$} \\
\hline Multidisciplinary team & $42(31.6 \%)$ \\
\hline Paediatrician & $39(29.3 \%)$ \\
\hline Speech and Language Therapist & $2(1.5 \%)$ \\
\hline Occupational Therapist & $2(1.5 \%)$ \\
\hline Clinical Psychologist & $17(12.8 \%)$ \\
\hline Educational Psychologist & $6(4.5 \%)$ \\
\hline Psychiatrist & $10(7.5 \%)$ \\
\hline Neurologist & $4(3.0 \%)$ \\
\hline Unsure or don't know & $10(7.5 \%)$ \\
\hline Other & $1(0.8 \%)$ \\
\hline Total & $133(82.1 \%)$ \\
\hline Missing & $29(17.9 \%)$ \\
\hline Child' age at the time of diagnosis of & $6.93(4.096),(18,1)$ \\
\hline Total & $133(82.1 \%)$ \\
\hline Missing & $29(17.9 \%)$ \\
\hline $\begin{array}{l}\text { Time taken between first seeking help } \\
\text { and receiving a diagnosis of ASD (in } \\
\text { months), } M(S D) \text {, (Max, Min) }\end{array}$ & $46.27(39.96)(216,5)$ \\
\hline Total & $132(81.5 \%)$ \\
\hline Missing & $30(18.5 \%)$ \\
\hline \multicolumn{2}{|l|}{$\begin{array}{l}\text { Receiving a written report on the } \\
\text { diagnosis, } n(\%)\end{array}$} \\
\hline Yes & $109(82.0 \%)$ \\
\hline No & $15(11.3 \%)$ \\
\hline Not sure & $9(6.8 \%)$ \\
\hline Total & $133(82.1 \%)$ \\
\hline Missing & $29(17.9 \%)$ \\
\hline \multicolumn{2}{|l|}{$\begin{array}{l}\text { Follow-up appointment with the same } \\
\text { professional following the diagnosis, } n \\
(\%)\end{array}$} \\
\hline Yes & $56(42.1 \%)$ \\
\hline No & $73(54.9 \%)$ \\
\hline Not sure & $4(3.0 \%)$ \\
\hline Total & $133(82.1 \%)$ \\
\hline Missing & $29(17.9 \%)$ \\
\hline
\end{tabular}




\section{Offering practical help/support by}

health professionals during the process

of seeking a diagnosis, $n(\%)$

Yes, offered directly (i.e., if assistance

$22(16.5 \%)$

was provided by, for example, arranging

appointments for you)

Yes, signposted towards (i.e., if

$41(30.8 \%)$

information was provided but it was left

to you to arrange contact)

No

$59(44.4 \%)$

Not sure

$6(4.5 \%)$

Other

$5(3.8 \%)$

Total

$133(82.1 \%)$

Missing

$29(17.9 \%)$

Offering practical help/support by

health professionals in follow-up

appointments after diagnosis, $\mathrm{n}(\%)$

Yes, offered directly (i.e., if assistance

$18(13.5 \%)$

was provided by, for example, arranging

appointments for you)

Yes, signposted towards (i.e., if

$43(32.3 \%)$

information was provided but it was left

to you to arrange contact)

No

Not sure

$62(46.6 \%)$

Other

$6(4.5 \%)$

Total

$4(3.0 \%)$

$133(82.1 \%)$

Missing

$29(17.9 \%)$

Satisfaction with the information given at diagnosis, $n(\%)$

Very dissatisfied

Quite dissatisfied

$28(21.1 \%)$

Neither satisfied nor dissatisfied

$35(26.3 \%)$

Quite satisfied

$32(24.1 \%)$

Very satisfied

$12(9.0 \%)$

Total

$133(82.1 \%)$

Missing

$29(17.9 \%)$

Stress during the diagnostic process, $n$

(\%)

Very stressful

$91(68.4 \%)$

Quite stressful

$29(21.8 \%)$

Not very stressful

$10(7.5 \%)$

Not at all stressful

$3(2.3 \%)$

Total

$133(82.1 \%)$

Missing

$29(17.9 \%)$ 


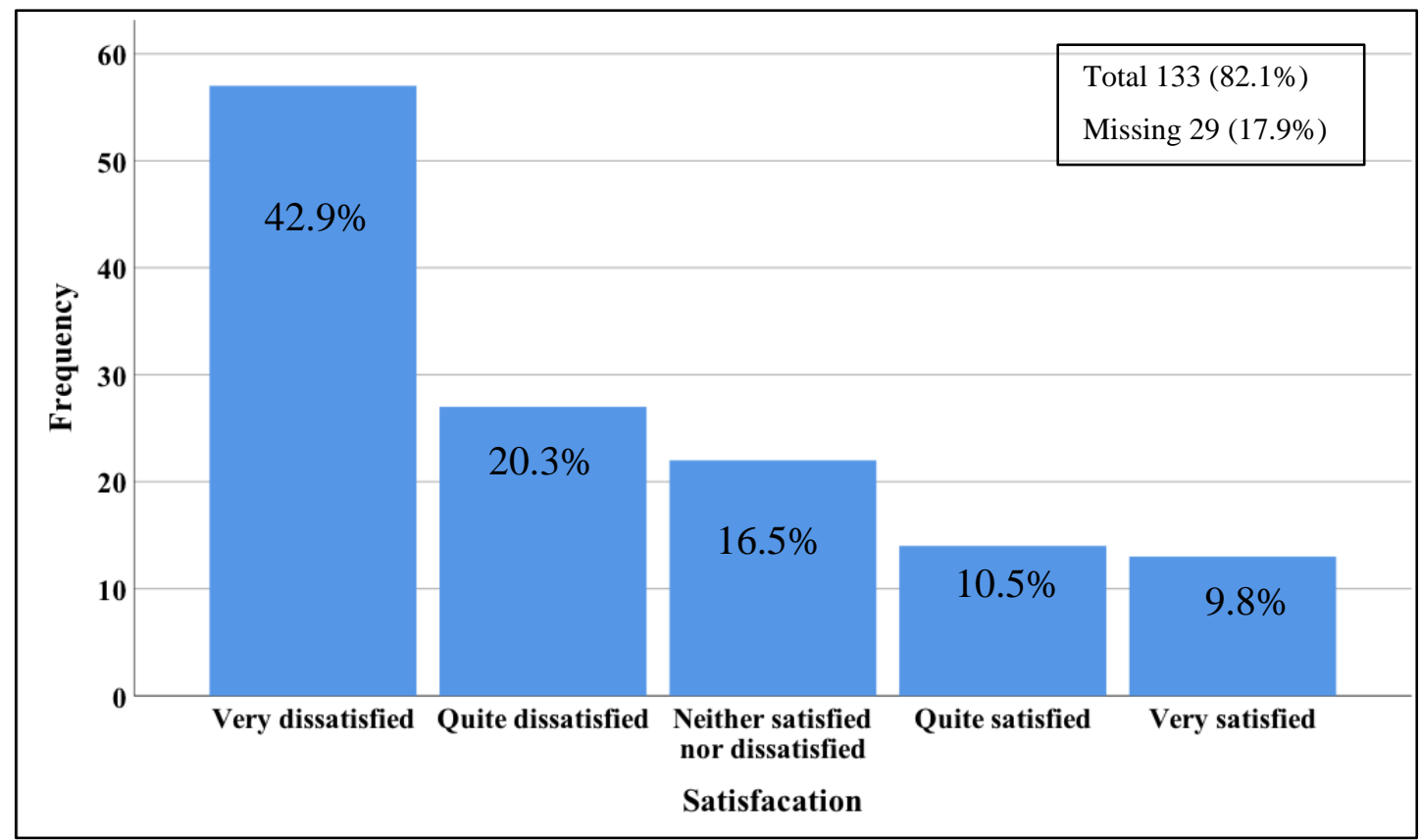

Figure 6-3: Parents/carers satisfaction with the time taken between first seeking help and receiving a diagnosis of $A S D$

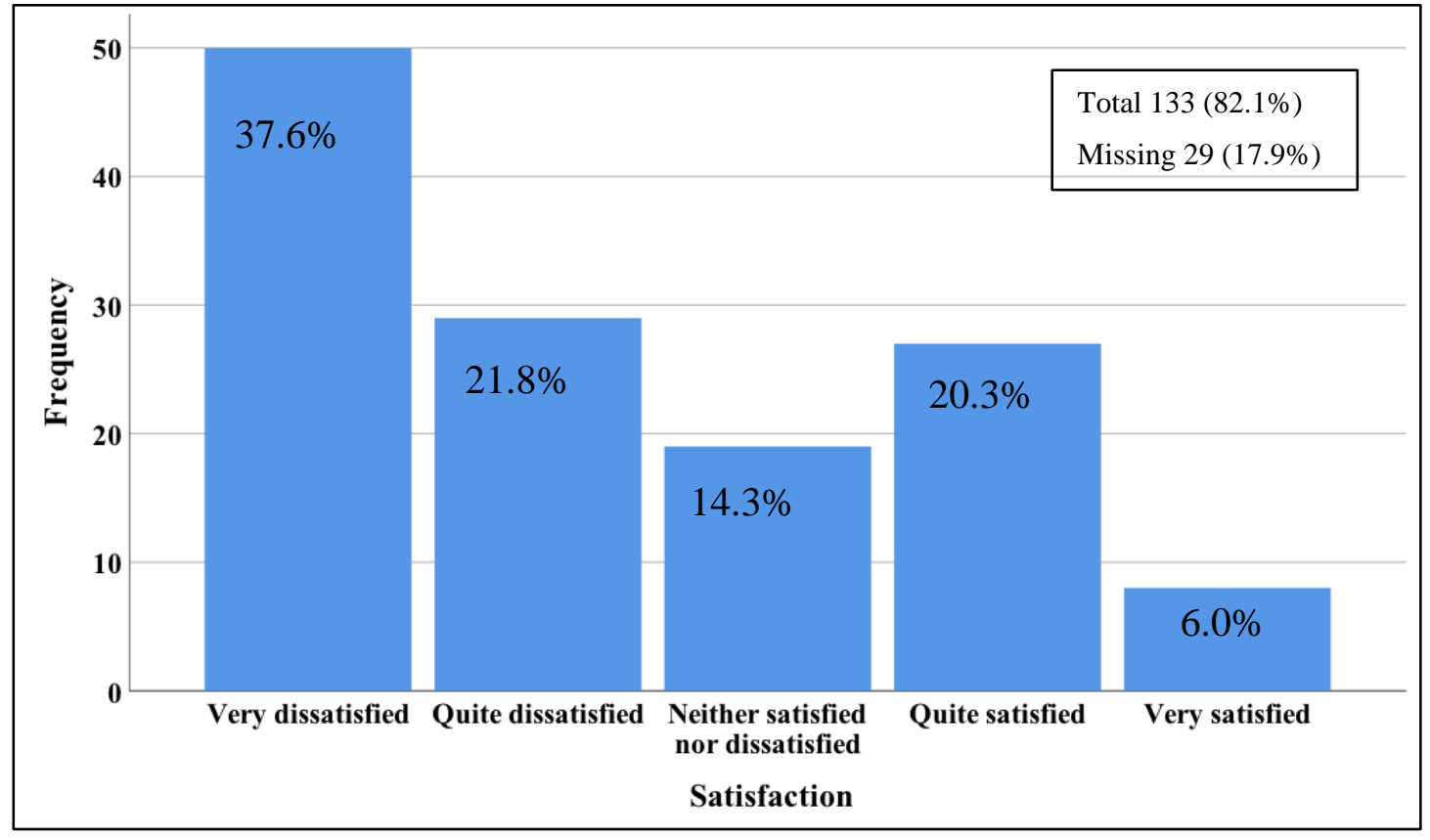

Figure 6-4:Parents/carers satisfaction with the diagnostic process as whole 


\subsection{Post-diagnostic support}

In terms of post-diagnostic support, some parents/carers reported that they had been offered a Statement of Special Educational Needs (SEN) (30\%), an explanation of their child's problems (23.5\%) and general advice on management (22.5\%) (see Table 6-5). Among parents/carers who were provided free with text responses to "other help/support" (28.3\%), some responses were related to receiving courses such as Early Bird:

"Referral to National Autistic Society (NAS) Early Bird programme." (Mother of a 13-year- old boy, diagnosed with Autism at the age of 3 years)

"Early Bird course and then nothing."

(Mother of a 13-year- old boy, diagnosed with Autism at the age of 4 years).

Some participants mentioned they were offered just a list of organisations, websites or out of date information:

"A badly photocopied sheet of paper with out of date information."

(Mother of a 12-year- old boy, diagnosed with Autism at the age of 11 years).

Other respondents reported that they received positive help/support:

"Was given a book on diagnosis, local ASD specialist got in contact thankfully \& helped."

(Father of a 12-year- old boy, diagnosed with ASD at the age of 10 years).

"Support from educational psychologist, head teacher, support staff, outreach support staff."

(Mother of a 15-year- old boy, diagnosed with Asperger syndrome at the age of 9 years).

"A list of useful websites, numbers, support groups."

(Mother of a 9-year- old boy, diagnosed with ASD at the age of 9 years).

However, some parents/carers stated that they did not receive any help/support:

"No help, told was only a diagnostic team and they did not do anything else or have places they could sign post me for her age."

(Mother of a 16-year- old girl, diagnosed with autism at the age of 15 years) 
"None offered."

(Mother of an 9-year- old girl, diagnosed with Autism at the age of 7 years)

"None, not seen by anyone since."

(Mother of an 8-year- old boy, diagnosed with ASD at the age of 7 years).

In response to the question "Overall, how satisfied were you with the help/support offered after your child was diagnosed?" the majority of those who responded to this question reported that they were not satisfied (63.6\%) and only $18.2 \%$ were satisfied.

Table 6-5: Post-diagnostic support

\begin{tabular}{ll}
\hline $\begin{array}{l}\text { Sort of help/support offered after child was } \\
\text { diagnosed, } \mathbf{n}(\%)\end{array}$ \\
Help with Nursery (pre/school) provision & $18(15.0 \%)$ \\
Statement of Special Educational Needs & $36(30 \%)$ \\
(SEN) & \\
Practical management (portage) & $10(8.3 \%)$ \\
Help with monetary benefits & $10(8.3 \%)$ \\
Help with medical problems & $11(9.2 \%)$ \\
Contact with other parents & $26(21.7 \%)$ \\
Language therapy & $24(20.0 \%)$ \\
Respite care & $3(2.5 \%)$ \\
General advice on management & $27(22.5 \%)$ \\
Personal support/counselling & $4(3.4 \%)$ \\
Family therapy & $3(2.5 \%)$ \\
Contact with a charity & $26(21.8 \%)$ \\
Explanation of child's problems & $28(23.5 \%)$ \\
Other & $34(28.3 \%)$ \\
Total & $120(74.1 \%)$ \\
Missing & $42(25.9 \%)$ \\
\hline Satisfaction with the help/support offered & \\
after child was diagnosed, n (\%) & \\
Very dissatisfied & $44(33.3 \%)$ \\
Quite dissatisfied & $40(30.3 \%)$ \\
Neither satisfied nor dissatisfied & $24(18.2 \%)$ \\
Quite satisfied & $15(11.4 \%)$ \\
Very satisfied & $9(6.8 \%)$ \\
Total & $132(81.5 \%)$ \\
Missing & $30(18.5 \%)$ \\
\hline
\end{tabular}




\subsection{Access to services}

With regards to access to services, the majority of the participants needed a referral to see any doctor, or receive any services for their child (78.5\%). Around 38\% of parents /carers reported that getting referrals was a big problem, $32.1 \%$ of them reported that it was a small problem and $19.1 \%$ reported it not being a problem. They indicated that they experienced difficulties/delays in accessing services for their children because they were not eligible for the services (58.8\%); services were not available (45.8\%); waiting lists, backlogs, or other problems getting appointments $(87.0 \%)$; lack of information (61.8\%) and cost (28.2\%). Over half of those responded reported that they had been always frustrated in their efforts to get services for their children $(52.0 \%)$ (see Table 6-6). Some respondents provided free text responses to other experienced difficulties (40.5\%). As mentioned earlier in the methodology chapter, a thematic analysis was conducted for the open-ended question "did you have any difficulties or delays for any other reason?" (see Pages 122, 129). The main themes with regard to these reasons for difficulties/delays, including the representative quotations as illustrative examples that cited by participants, were as follow (see Figure 6-5): 


\subsubsection{Reasons why parents/carers experienced difficulties/delays in accessing ASD related services:}

\section{a. Waiting time}

Some parents/carers reported that they had experienced difficulties in obtaining diagnostic-related services and some important tests such as Electroencephalography (EEG) due to long waiting times:

"3 years he has been waiting for an EEG."

(Mother of a 6-year- old boy, diagnosed with ASD at the age of 4 years)

"Everything took ages."

(Mother of a 11-year- old boy, diagnosed with Autism at the age of 10 years)

"My son had a huge regression and lost all of his speech and social communication skills. He was misdiagnosed with epilepsy and given medication before an EEG could be done to confirm the diagnosis. There was a 5 month wait to have an EEG done. We paid privately in the end and the test confirmed he did not have epilepsy. He was diagnosed a month later with autism."

(Mother of a 2-year- old boy, diagnosed with Autism at the age of 2 years)

"ASD seems to be a "secret" and I found all the aspects of this diagnosis exceptionally long. I fought nail and tooth to be taken seriously."

(Mother of a 7-year- old boy, diagnosed with ASD at the age of 7 years)

\section{b. Multiple referrals to different professionals}

The participants found that dealing with different professionals was one of the difficulties that they had faced:

"I was passed on a variety of professionals including an educational psychologist, an occupational therapist, locum paediatricians...I raised concerns but kept seeing different professionals so there was no continuity. Each professional referred me on to someone else. Auditory processing issues were seen as hearing problems. Fine motor skills were seen as something related to joint hypermobility. Speech problems were seen in isolation as relating to hypermobility. Toe 
walking was also treated by a podiatrist. No one (medically) was seeing the whole picture (except me)."

(Mother of a 9-year- old boy, diagnosed with Autism at the age of 7 years)

"Took 9 referrals through GP for CAMHS as he has severe anxiety and agoraphobia."

(Mother of a 7-year- old boy, diagnosed with ASD at the age of 7 years)

\section{c. Cancelled appointments}

Some parents/carers expressed that they had difficulties getting services because the appointments that were booked for their children were cancelled:

"Due to appointments being cancelled with no explanation." (Mother of a 9-year- old girl, diagnosed with Asperger syndrome at the age of 8 years)

"Lots of cancelled appointments."

(Mother of a 11-year- old boy, diagnosed with ASD at the age of 8 years)

\section{d. Lack of services and shortage of professionals}

The participants reported that another reason for difficulties was the significant lack of services and shortage of professionals:

"Did not see paediatrician for over a year as no doctor available." (Mother of a 11-year- old boy, diagnosed with ASD at the age of 8 years)

"No paediatrician employed in our area."

(Mother of a 14-year- old boy, diagnosed with Asperger syndrome at the age of 11 years)

"Nothing available."

(Mother of an 8-year- old boy, diagnosed with ASD at the age of 8 years) 
"Services are overworked or non-existent, at the best you can expect sparse appointments after long delays. Interacting with and obtaining services is so difficult, it is easier to manage with no support than go through the stress."

(Mother of a 10-year- old girl, diagnosed with ASD at the age of 3 years)

"No suitable counselling service despite doctor saying it was needed." (Mother of an 9-year- old girl, diagnosed with Autism at the age of 7 years)

"Fell between gaps in services because Asperger's does not count as a learning disability so no help available."

(Mother of a 15-year- old boy, diagnosed with Asperger syndrome at the age of 14 years)

\section{e. Inadequacies with Child and Adolescent Mental Health Services (CAMHS) provision}

Some participants had experienced delays due to issues relating to CAMHS such as a backlog, shortage of staff and disorganisation:

"First referral to CAMHS refused due to backlog and 7-year-old daughter not being suicidal or self-harming so not urgent case." (Mother of a 7-year- old girl, diagnosed with ASD at the age of 7 years)

"CAMHS very disorganised and short staffed."

(Mother of a 14-year- old boy, diagnosed with ASD at the age of 10 years)

"No one from CAMHS or neurodevelopment team saw my son as fell between age brackets."

(Mother of a 12-year- old boy, diagnosed with ASD at the age of 11 years) 


\section{f. Difficulties in finding the information}

Some parents/carers talked about the difficulty in obtaining the necessary information:

"Finding the information you need is ridiculously hard. There is no one stop shop \& no one point of contact to walk you through it all, which when your resources are already depleted is exhausting. Accessing services such as occupational therapist or respite is a lottery and extra work, when Ifeel it should be automatically offered following a diagnosis. You shouldn't have to fight for everything you need and prove your worthiness to have it."

(Mother of an 8-year- old boy, diagnosed with ASD at the age of 5 years)

\section{g. Issues related to professionals}

A number of parents/carers reported that they had difficulties obtaining services because of issues related to professionals (e.g. paediatrician, GP, health visitor, speech and language therapist, and educational psychologist) including a reluctance to listen to the concerns of the parent/carer, nonrecognition of parent/carer concerns, a delay in providing the child's Statement of Special Educational Needs (SEN), forgetting to make referrals, sending referrals to another professional with some errors and losing either paper work or assessments needed to make the diagnosis:

"Professionals unwilling to listen to concerns, as she is an intelligent girl she was seen as a child with behavioural issues, which they put down to the fact that I am single mother and she has no contact with her father."

(Mother of a 16-year- old girl, diagnosed with autism at the age of 15 years)

"Rather than working in partnership with parents or listening to child, professionals choose to be obstructive, having decided that I had invented my child's difficulties Fabricated or Induced Illness (FII) and even started devastating child protection, then changed back to child in need at first review."

(Mother of an 18-year- old girl, diagnosed with ASD at the age of 17 years)

"Speech and language therapy has been a constant battle, on his statement of SEN and did not receive it for the first year (I made 
complaints to the local authority). I have paid for private speech therapy in the past for a year."

(Mother of a 12-year- old boy, diagnosed with autism at the age of 4 years)

"Health visitor did not recognise my concerns. Said as a teacher I knew too much and was looking into things and he could give eye contact so definitely was not autistic!!!"

(Mother of a 9-year- old boy, diagnosed with autism at the age of 5 years)

"Professionals forgetting to do referrals... happened a few times... referrals going "missing"",

(Mother of a 4-year- old boy, diagnosed with autism at the age of 3 years)

"The paediatrician kept misplacing assessments needed to form 'official diagnosis 'after telling me at every appointment for 2 years he was on the spectrum"

(Mother of a 5-year- old boy, diagnosed with ASD at the age of 4 years)

"Errors with sending referral forms from the GP... they said they sent them... but the child development team had not received...... GP did not sign the form correctly etc. Chaos caused by forms going back and fore."

(Father of a 12-year- old boy, diagnosed with ASD at the age of 10 years)

"Lost paperwork by professionals and not accepted for a statement." (Mother of a 11-year- old boy, diagnosed with Autism at the age of 10 years)

"Hospital passed responsibility on to educational psychologist. Educational psychologist would not take notice as he was often late to school."

(Mother of a 7-year- old boy, diagnosed with ASD at the age of 7 years)

"From my experience, no matter what professionals see, they always believe that there is another reason to it before considering what my worries are, which is why the diagnosis time takes so long."

(Mother of a 6-year- old boy, diagnosed with ASD at the age of 5 years) 
In summary, these responses provided some insight into the difficulties faced by parents/carers in Wales when attempting to access services for their children. The parents/carers complained about the long waiting times. Some participants reported that they had to wait for certain tests e.g. EEG, for a very long time. Other parents had decided to pay privately to have a correct diagnosis rather than waiting any longer after receiving an inaccurate diagnosis. Multiple referrals to different professionals was also one of the barriers identified by participants. They also reported that they had difficulties because of issues pertaining to Child and Adolescent Mental Health Services (CAMHS) provision, highlighting staff shortage, a lack of organisation and backlogs. Cancelled appointments was another barrier that impacted on their ability to see professionals, with appointments being cancelled without explanation. Furthermore, they reported a shortage of the required services and specialist professionals e.g. a paediatrician was not available in some areas. Some parents/carers reported that they experienced delays due to issues related to professionals. Overall, it can be concluded that the parents of children with ASD in Wales, face considerable barriers when attempting to accessing services.

Table 6-6: Access to services

\begin{tabular}{ll}
\hline $\begin{array}{l}\text { Needing a referral to see any doctors or } \\
\text { receive any services for child, } \boldsymbol{n}(\boldsymbol{\%})\end{array}$ \\
Yes & $103(78.5 \%)$ \\
No & $21(16.0 \%)$ \\
Don't know & $7(5.3 \%)$ \\
Total & $131(80.9 \%)$ \\
Missing & $31(19.1 \%)$ \\
\hline Getting referrals, a big problem, a small & \\
problem, or not a problem, $\boldsymbol{n}(\boldsymbol{\%})$ & \\
Big problem & $50(38.2 \%)$ \\
Small problem & $42(32.1 \%)$ \\
Not a problem & $25(19.1 \%)$ \\
Don't know & $14(10.7 \%)$ \\
Total & $131(80.9 \%)$ \\
Missing & $31(19.1 \%)$ \\
\hline Difficulties or delays due to ineligibility for & \\
services, $\boldsymbol{n}(\%)$ & $77(58.8 \%)$ \\
Yes & $41(31.3 \%)$ \\
No & $13(9.9 \%)$ \\
Don't know &
\end{tabular}




\begin{tabular}{|c|c|}
\hline $\begin{array}{l}\text { Total } \\
\text { Missing }\end{array}$ & $\begin{array}{l}131(80.9 \%) \\
31(19.1 \%)\end{array}$ \\
\hline \multicolumn{2}{|c|}{$\begin{array}{l}\text { Difficulties or delays because of issues } \\
\text { related to cost, } n(\%)\end{array}$} \\
\hline Yes & $37(28.2 \%)$ \\
\hline No & $69(52.7 \%)$ \\
\hline Don't know & $25(19.1 \%)$ \\
\hline Total & $131(80.9 \%)$ \\
\hline Missing & $31(19.1 \%)$ \\
\hline \multicolumn{2}{|c|}{$\begin{array}{l}\text { Difficulties or delays due to waiting lists, } \\
\text { backlogs, or other problems getting } \\
\text { appointments, } n(\%)\end{array}$} \\
\hline Yes & $114(87.0 \%)$ \\
\hline No & $13(9.9 \%)$ \\
\hline Don't know & $4(3.1 \%)$ \\
\hline Total & $131(80.9 \%)$ \\
\hline Missing & $31(19.1 \%)$ \\
\hline \multicolumn{2}{|c|}{$\begin{array}{l}\text { Difficulties or delays because of lack of } \\
\text { services, } n(\%)\end{array}$} \\
\hline Yes & $60(45.8 \%)$ \\
\hline No & $53(40.5 \%)$ \\
\hline Don't know & $18(13.7 \%)$ \\
\hline Total & $131(80.9 \%)$ \\
\hline Missing & $31(19.1 \%)$ \\
\hline \multicolumn{2}{|c|}{$\begin{array}{l}\text { Difficulties or delays due to lack of } \\
\text { information, } n(\%)\end{array}$} \\
\hline Yes & $81(61.8 \%)$ \\
\hline No & $38(29.0 \%)$ \\
\hline Don't know & $12(9.2 \%)$ \\
\hline Total & $131(80.9 \%)$ \\
\hline Missing & $31(19.1 \%)$ \\
\hline \multicolumn{2}{|c|}{$\begin{array}{l}\text { Any difficulties or delays for any other } \\
\text { reason, } n(\%)\end{array}$} \\
\hline Yes & $53(40.5 \%)$ \\
\hline No & $48(36.6 \%)$ \\
\hline Don't know & $30(22.9 \%)$ \\
\hline Total & $131(80.9 \%)$ \\
\hline Missing & $31(19.1 \%)$ \\
\hline \multicolumn{2}{|c|}{$\begin{array}{l}\text { Frustration in efforts to get services for } \\
\text { child, } n(\%)\end{array}$} \\
\hline Never & $5(3.9 \%)$ \\
\hline Sometimes & $24(18.9 \%)$ \\
\hline Usually & $30(23.6 \%)$ \\
\hline Always & $66(52.0 \%)$ \\
\hline Don't know & $2(1.6 \%)$ \\
\hline Total & $127(78.4 \%)$ \\
\hline Missing & $35(21.6 \%)$ \\
\hline
\end{tabular}




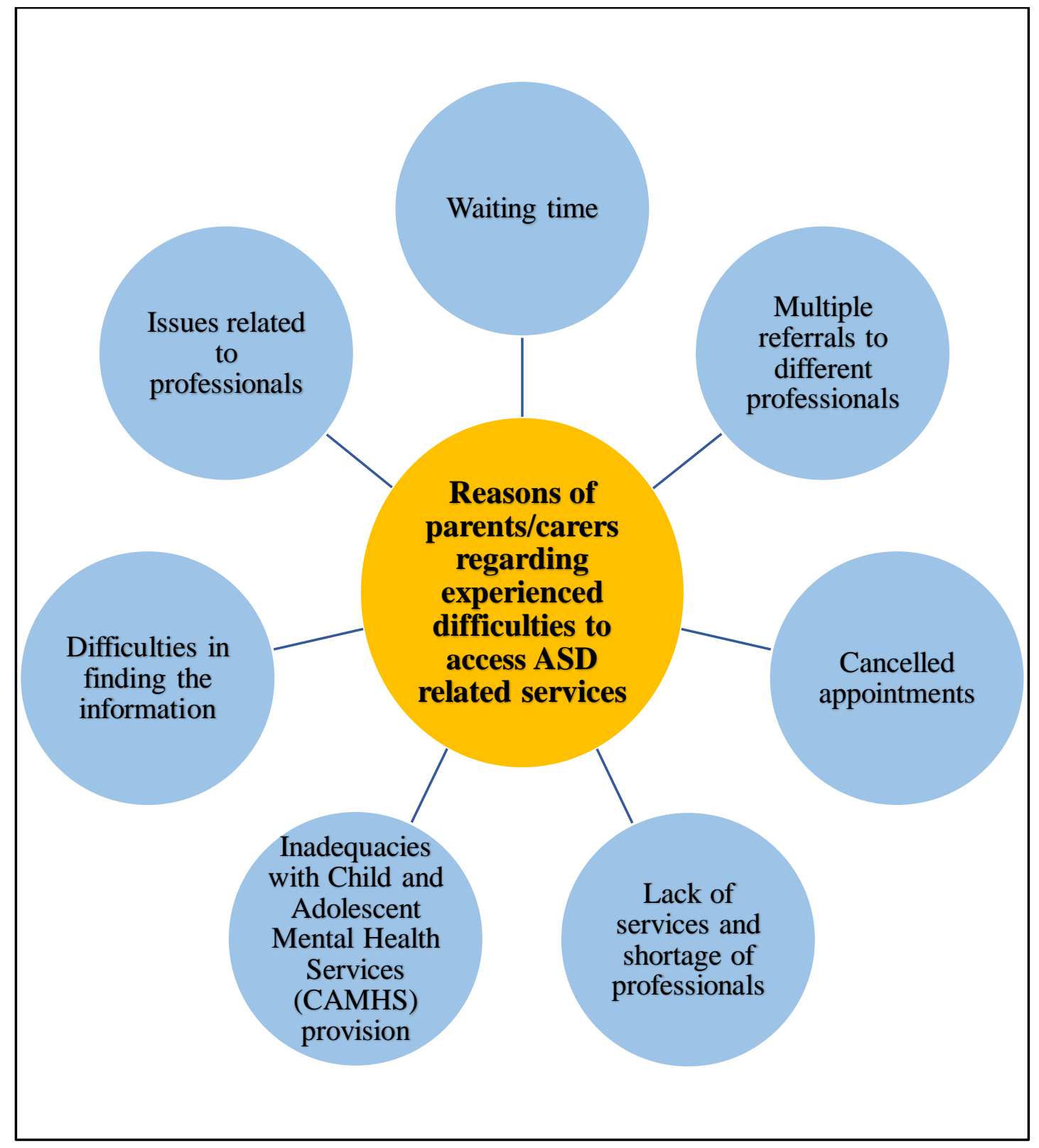

Figure 6-5: Reasons why parents/carers experienced difficulties/delays in accessing ASD related services 


\subsection{Factors associated with parent/carer satisfaction with the overall diagnostic process on univariate analysis}

As shown in Table 6-7, mainly diagnostic process related factors were univariately associated with a negative experience. These significant factors were the child's age when parents/carers began to have concerns (average 2.94 years (negative) vs. 2.20 years (not negative); difference 0.74 years; $95 \%$ CI [0.01 to 1.46]), child's age when parents/carers sought help (average 3.82 (negative) vs. 2.63 years (not negative); difference 1.19 years; 95\% CI [.37 to 2.01]), child's age at diagnosis (average 8.10 years (negative) vs. 5.22 years (not negative); difference 2.88 years; 95\% CI [1.53 to 4.23]), time elapsed from seeking help to receiving diagnosis (average 54.86 months (negative) vs. 33.87 months (not negative); difference 20.99 months; 95\% CI [7.42 to 34.56]), number of professionals consulted (proportion $73.42 \%$ (negative) vs. $53.70 \%$ (not negative); difference 19.72\%; 95\% CI [2\% to 36\%]), stress of the diagnostic process (proportion $98.73 \%$ (negative) vs. $77.78 \%$ (not negative); difference 20.95\%; $95 \%$ CI [10\% to $34 \%]$ ), satisfaction with the information given at diagnosis (proportion $79.75 \%$ (negative) vs. $47.17 \%$ (not negative); difference $32.58 \%$; $95 \%$ CI [16\% to $47 \%])$.

Regarding access to services related factors, getting referrals (proportion $77.92 \%$ (negative) vs. 59.26\% (not negative); difference $18.66 \%$; $95 \%$ CI [3\% to $34 \%]$ ), difficulties due to lack of services (proportion 53.25\% (negative) vs. 35.19\% (not negative); difference $18.06 \%$; $95 \%$ CI [ $1 \%$ to $34 \%$ ]) and difficulties due to lack of information (proportion $70.13 \%$ (negative) vs. 50\% (not negative); difference 20.13\%; $95 \%$ CI [3\% to $36 \%])$ were significant.

By contrast, no statistically significant associations were found between satisfaction and the parent/carer demographics, the child demographics, satisfaction with postdiagnostic help, difficulties accessing services due to ineligibility, difficulties because of issues related to cost or difficulties due to problems related to getting appointments and backlogs (see Table 6-7). 
Table 6-7: Univariate analysis of the association of variables on satisfaction with overall diagnostic process of ASD

\begin{tabular}{|c|c|c|c|c|}
\hline \multirow{2}{*}{ Variables } & \multicolumn{2}{|c|}{$\begin{array}{l}\text { Satisfaction with overall } \\
\text { diagnostic process }\end{array}$} & \multirow{2}{*}{$\begin{array}{c}\text { Mean/ } \\
\text { Proportion } \\
\text { Difference }\end{array}$} & \multirow{2}{*}{$95 \% \mathrm{CI}$} \\
\hline & $\begin{array}{c}\text { Negative } \\
\text { Experience }\end{array}$ & $\begin{array}{c}\text { Not } \\
\text { Negative } \\
\text { Experience } \\
\end{array}$ & & \\
\hline \multicolumn{5}{|l|}{$\begin{array}{l}\text { Parents'/Carers' } \\
\text { Demographics }\end{array}$} \\
\hline $\begin{array}{l}\text { Parent's/carer's age, } \\
M(S D)\end{array}$ & $\begin{array}{l}\mathrm{N}=78 \\
39.05 \\
(8.25)\end{array}$ & $\begin{array}{l}\mathrm{N}=54 \\
38.89 \\
(8.19)\end{array}$ & .16 & $(-2.72$ to 3.04$)$ \\
\hline $\begin{array}{l}\text { Parent's/carer's gender } \\
\text { Female, } \mathrm{n}(\%)\end{array}$ & $\begin{array}{c}\mathrm{N}=79 \\
77(97.47 \%)\end{array}$ & $\begin{array}{c}\mathrm{N}=54 \\
49(90.74 \%)\end{array}$ & $6.73 \%$ & $(-1 \%$ to $18 \%)$ \\
\hline $\begin{array}{l}\text { Currant marital status } \\
\text { Cohabit/married, n (\%) }\end{array}$ & $\begin{array}{c}\mathrm{N}=79 \\
61(77.22 \%)\end{array}$ & $\begin{array}{c}\mathrm{N}=54 \\
43(79.63 \%)\end{array}$ & $2.41 \%$ & $(-12 \%$ to $16 \%)$ \\
\hline $\begin{array}{l}\text { Living area } \\
\text { South Wales, } \mathrm{n}(\%)\end{array}$ & $\begin{array}{c}\mathrm{N}=79 \\
60(75.95 \%)\end{array}$ & $\begin{array}{c}\mathrm{N}=54 \\
37(68.52 \%)\end{array}$ & $7.43 \%$ & $(-8 \%$ to $23 \%)$ \\
\hline $\begin{array}{l}\text { Education level } \\
\text { Higher education, n (\%) }\end{array}$ & $\begin{array}{c}\mathrm{N}=79 \\
39(49.37 \%)\end{array}$ & $\begin{array}{c}\mathrm{N}=54 \\
21(38.89 \%)\end{array}$ & $10.48 \%$ & $(-5 \%$ to $27 \%)$ \\
\hline $\begin{array}{l}\text { Number of children, } \\
M(S D)\end{array}$ & $\begin{array}{l}\mathrm{N}=78 \\
2.47 \\
(1.17) \\
\end{array}$ & $\begin{array}{c}\mathrm{N}=53 \\
2.30 \\
(.87) \\
\end{array}$ & .17 & (-.20 to .55$)$ \\
\hline $\begin{array}{l}\text { Number of children } \\
\text { have autism-ASD, } M \\
(S D)\end{array}$ & $\begin{array}{c}\mathrm{N}=78 \\
1.19 \\
(.43) \\
\end{array}$ & $\begin{array}{l}\mathrm{N}=53 \\
1.23 \\
(.51) \\
\end{array}$ & .04 & (-.20 to .13$)$ \\
\hline \multicolumn{5}{|l|}{ Child's Demographics } \\
\hline $\begin{array}{l}\text { Current child's age, } \\
M(S D)\end{array}$ & $\begin{array}{c}\mathrm{N}=79 \\
9.96 \\
(4.33) \\
\end{array}$ & $\begin{array}{c}\mathrm{N}=54 \\
8.70 \\
(3.86) \\
\end{array}$ & 1.26 & $(-.19$ to 2.71$)$ \\
\hline $\begin{array}{l}\text { Child's gender } \\
\text { Male, } \mathrm{n}(\%)\end{array}$ & $\begin{array}{c}\mathrm{N}=79 \\
63(79.75 \%)\end{array}$ & $\begin{array}{c}\mathrm{N}=54 \\
40(74.07 \%)\end{array}$ & $5.68 \%$ & (-8\% to $21 \%)$ \\
\hline $\begin{array}{l}\text { Child's diagnosis } \\
\text { Autism Spectrum } \\
\text { Disorder, } \mathrm{n}(\%) \\
\end{array}$ & $\begin{array}{c}\mathrm{N}=77 \\
37(48.05 \%)\end{array}$ & $\begin{array}{c}\mathrm{N}=52 \\
24(46.15 \%)\end{array}$ & $1.9 \%$ & $(-15 \%$ to $19 \%)$ \\
\hline $\begin{array}{l}\text { Diagnostic Process } \\
\text { related Variables }\end{array}$ & & & & \\
\hline $\begin{array}{l}\text { Child's age when } \\
\text { parents began to have } \\
\text { concerns, } M(S D)\end{array}$ & $\begin{array}{c}N=79 \\
2.94 \\
(2.52)\end{array}$ & $\begin{array}{c}\mathrm{N}=54 \\
2.20 \\
(\mathbf{1 . 1 9})\end{array}$ & $.74 *$ & (.01 to 1.46$)$ \\
\hline $\begin{array}{l}\text { Child's age when } \\
\text { parents sought help, } \\
M(S D)\end{array}$ & $\begin{array}{c}\mathrm{N}=79 \\
3.82 \\
(2.77)\end{array}$ & $\begin{array}{c}N=54 \\
2.63 \\
(1.53)\end{array}$ & $1.19 *$ & (.37 to 2.01$)$ \\
\hline $\begin{array}{l}\text { Child's age at diagnosis, } \\
M(S D)\end{array}$ & $\begin{array}{c}N=79 \\
8.10 \\
(4.21)\end{array}$ & $\begin{array}{c}\mathrm{N}=54 \\
5.22 \\
(3.27)\end{array}$ & $2.88 *$ & (1.53 to 4.23$)$ \\
\hline
\end{tabular}




\begin{tabular}{|c|c|c|c|c|}
\hline $\begin{array}{l}\text { Time taken to get } \\
\text { diagnosis, } M(S D)\end{array}$ & $\begin{array}{c}N=78 \\
54.86 \\
(43.10) \\
\end{array}$ & \begin{tabular}{c|c|}
$N=54$ \\
33.87 \\
$(31.33)$ \\
\end{tabular} & 20.99* & (7.42 to 34.56$)$ \\
\hline $\begin{array}{l}\text { Number of } \\
\text { professionals consulted } \\
\text { Four and more } \\
\end{array}$ & $\begin{array}{c}\mathrm{N}=79 \\
58(73.42 \%)\end{array}$ & $\begin{array}{c}\mathrm{N}=54 \\
29(53.70 \%)\end{array}$ & $19.72 \% *$ & $(2 \%$ to $36 \%)$ \\
\hline $\begin{array}{l}\text { Stress of the diagnostic } \\
\text { process }\end{array}$ & $\mathrm{N}=79$ & $\mathrm{~N}=54$ & & \\
\hline Stressful, n (\%) & $78(98.73 \%)$ & $42(77.78 \%)$ & $20.95 \% *$ & $(10 \%$ to $34 \%)$ \\
\hline $\begin{array}{l}\text { Satisfaction with the } \\
\text { information given at } \\
\text { diagnosis } \\
\text { Dissatisfied, } \mathrm{n}(\%)\end{array}$ & $\begin{array}{c}N=79 \\
63(79.75 \%)\end{array}$ & $\begin{array}{c}N=53 \\
25(47.17 \%)\end{array}$ & $32.58 \% *$ & $(16 \%$ to $47 \%)$ \\
\hline $\begin{array}{l}\text { Satisfaction with the } \\
\text { post-diagnostic help } \\
\text { Dissatisfied, } \mathrm{n}(\%)\end{array}$ & $\begin{array}{c}\mathrm{N}=31 \\
24(77.42 \%)\end{array}$ & $\begin{array}{c}\mathrm{N}=30 \\
21(70 \%)\end{array}$ & $7.42 \%$ & $(-14 \%$ to $29 \%)$ \\
\hline $\begin{array}{l}\text { Access to Services } \\
\text { related Variables }\end{array}$ & & & & \\
\hline $\begin{array}{l}\text { Getting referrals } \\
\text { Problem, } \mathrm{n}(\%)\end{array}$ & $\begin{array}{c}N=77 \\
60(77.92 \%)\end{array}$ & $\begin{array}{c}\mathrm{N}=54 \\
32(59.26 \%)\end{array}$ & $18.66 \% *$ & $(3 \%$ to $34 \%)$ \\
\hline $\begin{array}{l}\text { Difficulties due to } \\
\text { ineligibility for services } \\
\text { Yes, } \mathrm{n}(\%)\end{array}$ & $\begin{array}{c}\mathrm{N}=77 \\
48(62.34 \%)\end{array}$ & $\begin{array}{c}\mathrm{N}=54 \\
29(53.70 \%)\end{array}$ & $8.64 \%$ & $(-8 \%$ to $25 \%)$ \\
\hline $\begin{array}{l}\text { Difficulties because of } \\
\text { issues related to cost } \\
\text { Yes, } \mathrm{n}(\%)\end{array}$ & $\begin{array}{c}\mathrm{N}=77 \\
23(29.87 \%)\end{array}$ & $\begin{array}{c}\mathrm{N}=54 \\
14(25.93 \%)\end{array}$ & $3.94 \%$ & (-12\% to $19 \%)$ \\
\hline $\begin{array}{l}\text { Difficulties due to } \\
\text { problems related to } \\
\text { getting appointments } \\
\text { Yes, n }(\%)\end{array}$ & $\begin{array}{c}\mathrm{N}=77 \\
70(90.91 \%)\end{array}$ & $\begin{array}{c}\mathrm{N}=54 \\
44(81.48 \%)\end{array}$ & $9.43 \%$ & $(-2 \%$ to $23 \%)$ \\
\hline $\begin{array}{l}\text { Difficulties due to lack } \\
\text { of services } \\
\text { Yes, } n(\%)\end{array}$ & $\begin{array}{c}\mathrm{N}=77 \\
\mathbf{4 1}(53.25 \%)\end{array}$ & $\begin{array}{c}\mathrm{N}=54 \\
19(35.19 \%)\end{array}$ & $18.06 \% *$ & $(1 \%$ to $34 \%)$ \\
\hline $\begin{array}{l}\text { Difficulties due to lack } \\
\text { of information } \\
\text { Yes, } \mathrm{n}(\%)\end{array}$ & $\begin{array}{c}\mathrm{N}=77 \\
54(70.13 \%)\end{array}$ & $\begin{array}{c}\mathrm{N}=54 \\
27(50 \%)\end{array}$ & $20.13 \% *$ & $(3 \%$ to $36 \%)$ \\
\hline
\end{tabular}




\subsection{Predictors of parent/carer satisfaction with the diagnostic process}

A binary logistic regression analysis (Pallant, 2016) was used to examine which factors were predictive of the overall satisfaction of parents/carers with the ASD diagnostic process. All of the variables were entered in the model. The least significant variable that does not meet the level for remaining in the model was removed. Once the variable is removed from the model, it remains excluded. The process is repeated until no other variables in the model meet the specified level for removal (Bursac et al., 2008; Pallant, 2016). The binary logistic regression was carried out twice, once with only observed data and once with imputed data. Overall satisfaction with the diagnostic process was used as the dependent variable and it was dichotomised to a negative experience and not negative experience with the overall diagnostic process. Predictor variables are shown in Table 6-8. As we stated in Chapter 5, some categories were collapsed because there was limited number of responses in some groups (Pallant, 2016).

\subsubsection{Assumptions of the binary logistic regression}

Before conducting the binary logistic regression, the assumptions of the logistic regression analysis (Pallant, 2016) were tested. These were as follows:

1. A dichotomous dependent variable.

The variable satisfaction with the overall diagnostic process was recorded into a dichotomous variable: not a negative experience with the overall diagnostic process (combining very satisfied, quite satisfied, and neither satisfied nor dissatisfied) and a negative experience with the overall diagnostic process (combining very dissatisfied and quite dissatisfied).

2. Absence of multicollinearity.

The absence of multicollinearity is the assumption that the predictor variables are not highly correlated, and in this study, was assessed by using 'Tolerance'. Tolerance values that are very low (less than .1) indicate that the variable has high correlation with other variables (see Page 127). We found two correlated 
predictors: a child's age at time of diagnosis and the time taken to obtain a diagnosis. The Tolerance value of the child's age at diagnosis variable was (.060) which is less than a value of the time taken to obtain a diagnosis variable (.063). Therefore, the child's age at diagnosis variable was removed. The test then was repeated and none of the tolerance values of the predictor variables was less than .1.

3. Absence of outliers

Although there was some indication of outlying cases that identified by 'Casewise List' (Pallant, 2016) (see Page 127), these cases were fully investigated and nothing abnormal was found in the outlier values. Therefore, these cases were kept, as outlier data are not always considered as errors (Rousseeuw \& Hubert, 2018).

\subsubsection{Parent/carer satisfaction with the overall diagnostic process of ASD model (with missing data)}

Using a binary logistic regression model, characteristics differentiating parents/carers who had a negative experience from those who had a not negative experience with the diagnostic process of ASD were investigated (see Table 6-8). Time taken to get diagnosis was incorporated into the model. Omnibus Tests of Model Coefficients was used, including Chi-Square, Degrees of Freedom (df) and p-value to check that the new model is an improvement over the baseline model. The purpose of the p-value in this test is to determine if the overall model is statistically significant. The results showed that full model containing the one predictor was significant, [Chi-Square = 9.972, $d f=1$ and $p=0.002(<0.05)]$, indicating that the model was able to distinguish between those parents/carers who reported a negative experience with the overall diagnostic process, and those who did not. The one predictor was significant at the 5\% level. 
The odds ratio is the probability that an event will occur divided by the probability that an event will not occur. The odds ratio is a ratio and this means is a comparison between how often an event occurs compared to how often it does not occur. It does not reflect how often and event is actually likely to occur (e.g. $2 \%$ of the time), but is simply shows that there is a higher chance of the event happening compared to not happening. In this case a higher chance the person will report a negative experience (compared to a positive/neutral experience) for each month delay.

Odds Ratio are the chance that a person who is exposed (e.g. delay in diagnosis) will be report a negative experience. For every additional month of exposure (e.g. delay in diagnosis) above the baseline, the chance that the person will report a negative experience increased by 1.016 times (e.g. baseline x 1.016 x number of months delayed/exposed).

The model correctly predicted $48.1 \%$ of cases where there was a not negative experience and $76.9 \%$ where there was a negative experience, giving an overall percentage correct prediction rate of $65.2 \%$. The Hosmer-Lemeshow test was used to determine the goodness of fit of the model, with a p-value $>0.05$ indicating that the final model was a good fit for the data (chi-square: $13.497, \mathrm{df}=8, p=.096)$. The final model as whole explained between $73 \%$ (Cox and Snell R Square) and 98\% (Nagelkerke R Square) of the variance in parent/carer satisfaction with the overall diagnostic process.

Table 6-8: Binary Logistic Regression Model (with missing data) of Parents/carers satisfaction with the overall diagnostic process of ASD model

\begin{tabular}{lccccc}
\multicolumn{1}{c}{ Variable } & Beta & SE & Wald & OR $(95 \%$ CI $)$ & $P$-value \\
\hline $\begin{array}{l}\text { Time taken to get } \\
\text { diagnosis }\end{array}$ & .016 & .006 & 8.020 & $1.016(1.005-.1 .028)$ & $.005^{*}$ \\
\end{tabular}

Note. Variables examined include; parent'/carer' age, parent'/carer' gender, marital status, educational level, living area, number of children, number of children with ASD, current child's age, child's age when parents began to have concerns, child's age when parents sought help, child's gender, time taken between first seeking help and receiving a diagnosis of ASD, child's current diagnosis, stress during the diagnostic process, satisfaction with the information given at diagnosis, satisfaction with postdiagnostic help, getting referrals, difficulties due to ineligibility, difficulties because of issues related to cost, difficulties because of issues related to waiting lists and backlogs, difficulties due to lack of services, difficulties due to lack of information.

$O R=$ Odds Ratio CI = confidence interval

${ }^{*} P<.05$ 


\subsubsection{Parent/carer satisfaction with the overall diagnostic process of ASD model (after imputation)}

The binary logistic regression was carried out with imputed data. As previously mentioned in Chapter 5, the missing values were assumed to be missing at random (MAR) which means that any difference between the observed values and the missing values might be described by differences in observed data (The Sterne et al., 2009). This is because that the parents/carers maybe did not have enough time to complete the questionnaire. Therefore, the missing values of the dependent and independent variables that were included in the previous logistic regression model, were imputed by using multiple imputation (see Page 128). As presented in Table 6-9 the result was still the same when the binary logistic regression was carried out after imputation. Imputation did not change the findings suggesting data was missing completely at random and so missing data has the same distributions as the observed data and imputation will only increase sample size rather than needing to account for systemic differences in missingness.

Table 6-9: Binary Logistic Regression Model (with imputed data) of parents/carers satisfaction with the overall diagnostic process of ASD model

\begin{tabular}{lccccc}
\hline \multicolumn{1}{c}{ Variable } & Beta & SE & Wald & OR $(95 \%$ CI $)$ & $P$-value \\
\hline $\begin{array}{l}\text { Time taken to get } \\
\text { diagnosis }\end{array}$ & .016 & .006 & 8.020 & $1.016(1.005-.1 .028)$ & $.005^{*}$ \\
\end{tabular}

Note. Variables examined include; parent'/carer' age, parent'/carer' gender, marital status, educational level, living area, number of children, number of children with ASD, current child's age, child's age when parents began to have concerns, child's age when parents sought help, child's gender, time taken between first seeking help and receiving a diagnosis of ASD, child's current diagnosis, stress during the diagnostic process, satisfaction with the information given at diagnosis, satisfaction with postdiagnostic help, getting referrals, difficulties due to ineligibility, difficulties because of issues related to cost, difficulties because of issues related to waiting lists and backlogs, difficulties due to lack of services, difficulties due to lack of information.

$O R=$ Odds Ratio CI = confidence interval

${ }^{*} P<.05$ 


\subsection{Summary}

This chapter presented the results of Study (2) that aimed to explore the experience of parents/carers in Wales with the diagnostic process of ASD, examining both the predictors and the significant factors associated with parent/carer satisfaction with the overall diagnostic process. After data cleaning, 162 cases were eligible for analysis. The chapter presented some descriptive data of the demographics of parents/carers, characteristics of the child, diagnostic process of ASD, final diagnosis and satisfaction, post-diagnostic support and access to services difficulties. Univariate analysis was conducted to determine the variables associated with parent/carer satisfaction with the diagnostic process of ASD. Binary logistic regression was also performed to determine the extent of likelihood of the predictors of the satisfaction with overall diagnostic process.

\section{Key findings to be taken forward:}

- On average, parents/carers face a delay of 46.27 months between seeking help and obtaining a diagnosis of ASD.

- Majority of the parents/carers were dissatisfied with the time taken to get diagnosis $(63.2 \%)$.

- Around $40 \%$ of the participants were dissatisfied with the information given at diagnosis.

- It is crucial to indicate that the majority of parents/carers were dissatisfied (59.4\%) with the diagnostic process as a whole.

- Majority of the parents/carers felt the diagnostic process was very stressful $(68.4 \%)$.

- Majority of the parents/carers were dissatisfied with the help/support offered after diagnosis $(63.6 \%)$.

- Parents/carers may experience difficulties/ delays in accessing services for their children because of ineligibility for services $(58.8 \%)$; unavailability of services (45.8\%); waiting lists and backlogs (87.0\%); lack of information (61.8\%) and cost (28.2\%). Waiting lists and backlogs was the most reported issue.

- Some respondents identified other difficulties in accessing services, such as multiple referrals to different professionals, cancelled 
appointments, shortage of services and specialist professionals, inadequacies with Child and Adolescent Mental Health Services (CAMHS) provision, lack of appropriate information and some issues related to professionals.

- The univariate analysis identified that the following variables were associated with parents/carers satisfaction: child's age when parents/carers began to have concerns, child's age when parents/carers first sought help, child's age at time of diagnosis, time taken from seeking help to obtaining a diagnosis, stress of the diagnostic process, getting referrals, number of professionals consulted, satisfaction with information given at diagnosis, difficulties due to lack of services and difficulties arising from a lack of information.

- The binary logistic regression analysis model revealed that time taken to get diagnosis play a main role in the degree of parent/carer satisfaction with the overall diagnostic process. In other words, a delay in receiving a diagnosis, which might hinder efforts to access support and early intervention services, may lead to increased parent/carer dissatisfaction. Therefore, more work is required to enable families to access a timely diagnosis.

- The Hosmer and Lemeshow goodness-of-fit test suggested that the model was a good fit for the data (chi-square: 13.497, $p=.096)$. The model as whole explained between $73 \%$ (Cox and Snell R Square) and 98\% (Nagelkerke R Square) of the variance in parent/carer satisfaction with the overall diagnostic process.

- Taken together, these results indicate that parents/carers of children with ASD in Wales face considerable barriers in gaining access to services. Most of parents/carers were dissatisfied with the ASD diagnostic process as whole. Evidently, more needs to be done to improve the parent/carer experiences of the diagnostic process for ASD children and enable families to receive diagnosis earlier.

As outlined in Chapter 3, telehealth technology could potentially play a positive role in the diagnosis and assessment of ASD. With reference to NODA, it has the potential to improve the diagnostic process of ASD. However, the successful implementation 
of telehealth is highly reliant on user acceptance (Davis, 1993; Hu et al., 1999). The next Chapter therefore, moves on to discuss the parent/carer acceptance of using NODA for ASD diagnostic assessment and to determine the predictors of their behavioural intention to use such technology. 


\section{Chapter 7: Study (3) Results: Exploring Parents/Carers Acceptance of Using NODA for ASD Diagnostic Assessment for their Children in Wales}

\subsection{Introduction}

The purpose of this chapter is to present the Study 3 findings of the data collected by the methods described in Chapter 5. The previous chapter has indicated that the majority of parents/carers are dissatisfied with the ASD diagnostic process in Wales as a whole. The NODA system has the potential to improve the diagnostic process, as was suggested by the scoping review conducted in Chapter 3. However, user acceptance is essential for successful implementation of telehealth (Davis, 1993; Hu et al., 1999). Therefore, this chapter will examine parent/carer acceptance of using NODA for ASD diagnostic assessment. Initially, the chapter presents the findings regarding the parent/carer experience with technology. Parent/carer perceptions on NODA are provided, using UTAUT/added constructs (performance expectancy, effort expectance, social influence, facilitating conditions, self-efficacy, and anxiety), along with the potential reasons why parents felt they would, or would not, use the NODA system. It then presents a projection of the expected usage of NODA for diagnostic assessment in Wales, if it had either been provided free by the NHS or privately. In addition, the chapter presents the results of the univariate analysis and binary logistic regression model (with missing data/with imputed data) of the factors that influence the acceptance of parents/carers in Wales to use NODA for diagnostic assessment. Results from the data analysis outlined in this chapter are used to provide the answer to the following research question:

What are the predictors of the intention of parents/carers in Wales, to use the NODA for ASD diagnostic assessment? 


\subsection{Demographics of the parents/carers}

(See Chapter 6 section 6.3)

\subsection{General information about the child with ASD}

(See Chapter 6 section 6.4)

\subsection{Technology Experience}

This section presents the findings related to the experience that parents/carers have with technology, including computer/internet skills, comfort with using a desktop computer, laptop computer, tablet computer and mobile phone/smartphone devices. Of the 113 participants who responded to this section, $58(51.3 \%)$ indicated that they have average computer/internet skills, 33 (29.2\%) rated themselves as advanced and only $3(2.7 \%)$ of respondents assessed themselves as novice (see Table 7-1). In response to the question: 'How comfortable are you with using the desktop computer?' over half of those who responded showed that they are very comfortable (52.2\%) (see Figure 7-1). Most of respondents also stated that they are very comfortable with using a laptop computer $(54.0 \%)$ and $48.7 \%$ of them reported that they are very comfortable with using a tablet computer (see Figure 7-2 \& Figure 7-3). The majority of those who responded $(69.9 \%)$ rated themselves as being very comfortable with using a mobile phone/smartphone (see Figure 7-4). This indicates that participants had a high level of confidence in using such technologies, particularly the mobile phone/smartphone.

Table 7-1: Parents'/carers' experience with technology

\begin{tabular}{ll}
\hline Computer/Internet Skills, $\boldsymbol{n}(\boldsymbol{\%})$ & \\
Novice (None) & $3(2.7 \%)$ \\
Basic & $17(15.0 \%)$ \\
Average & $58(51.3 \%)$ \\
Advance & $33(29.2 \%)$ \\
Expert & $2(1.8 \%)$ \\
Total & $113(69.8 \%)$ \\
Missing & $49(30.2 \%)$ \\
\hline
\end{tabular}




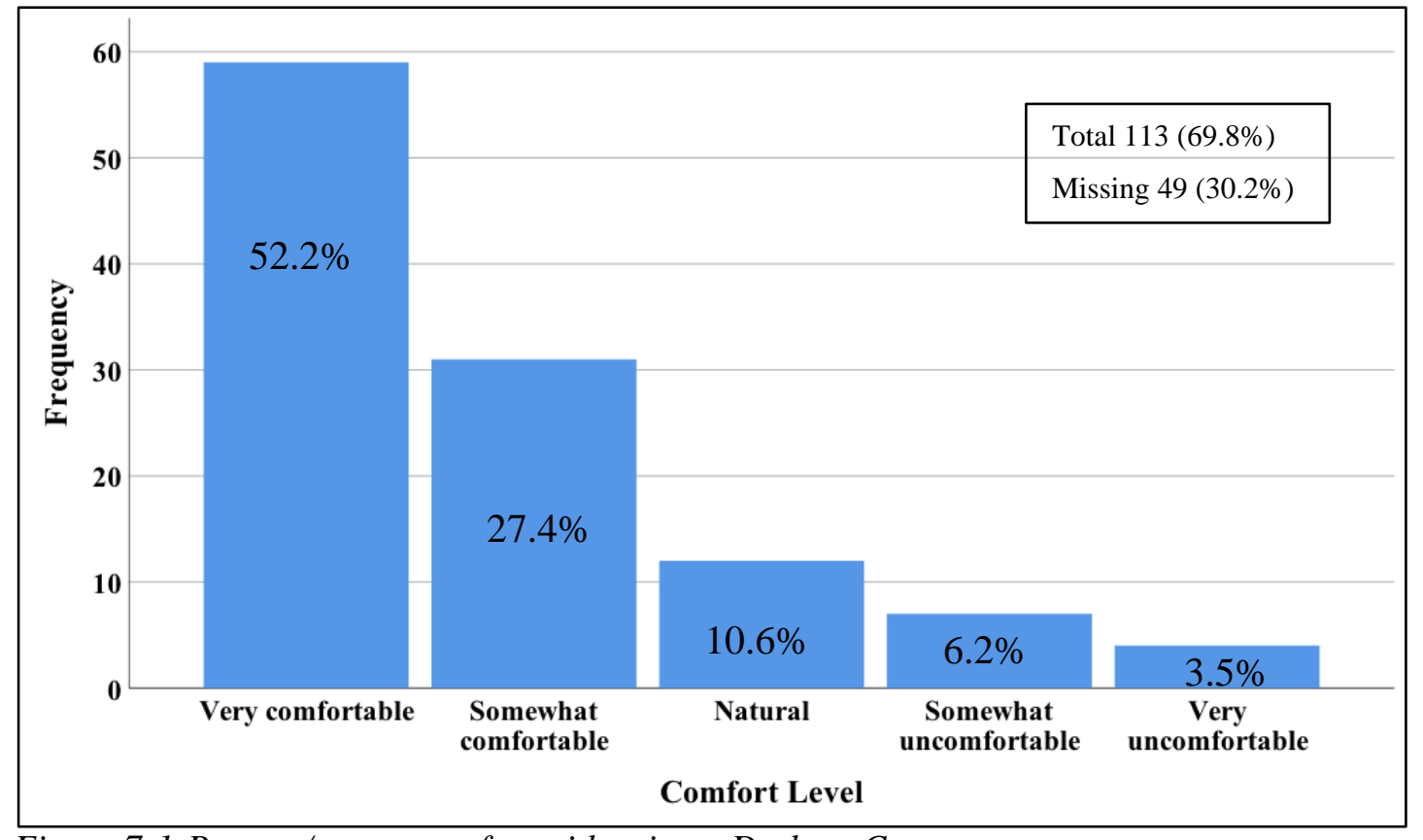

Figure 7-1:Parents/carers comfort with using a Desktop Computer

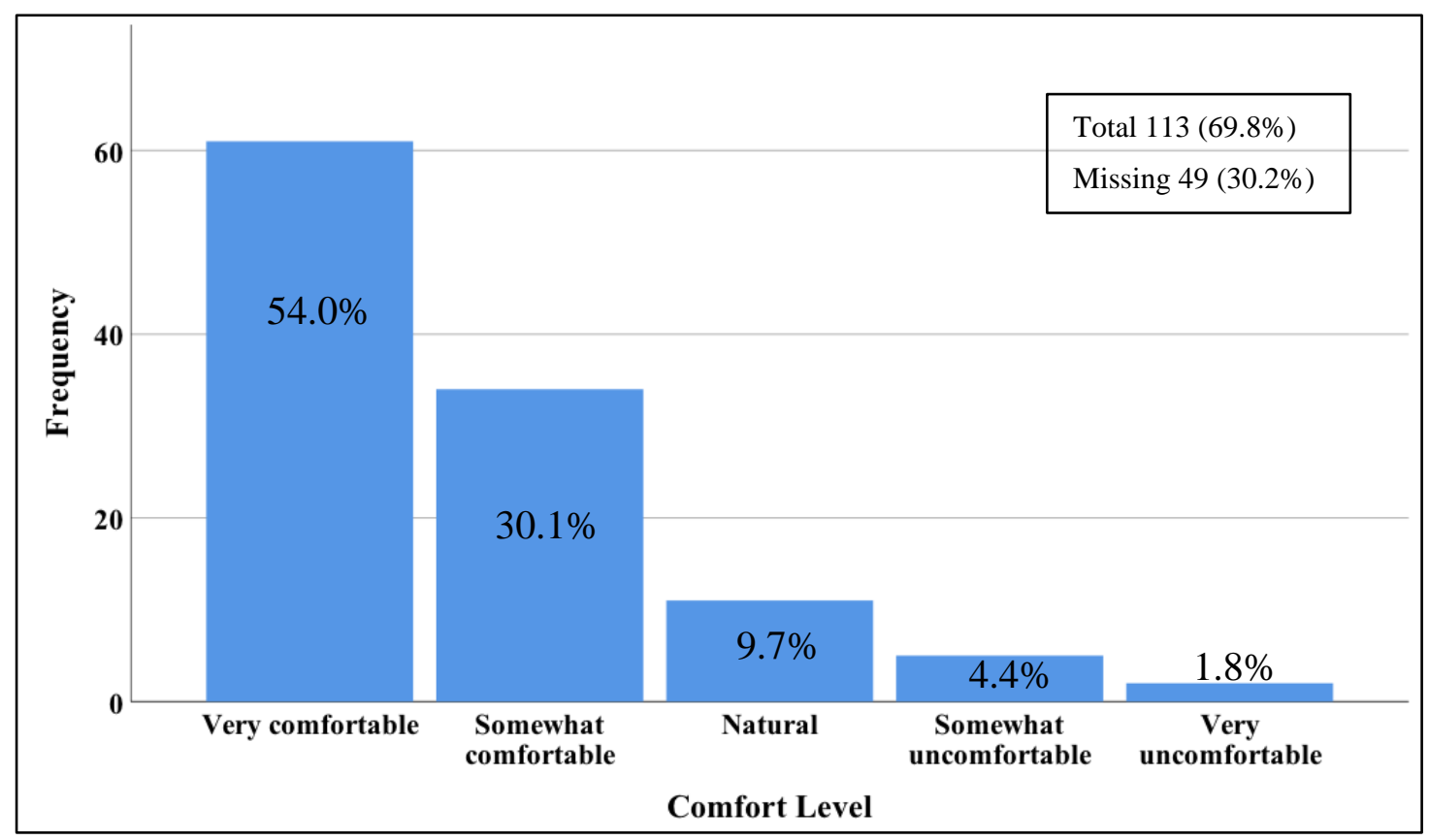

Figure 7-2: Parents/carers comfort with using a Laptop Computer 


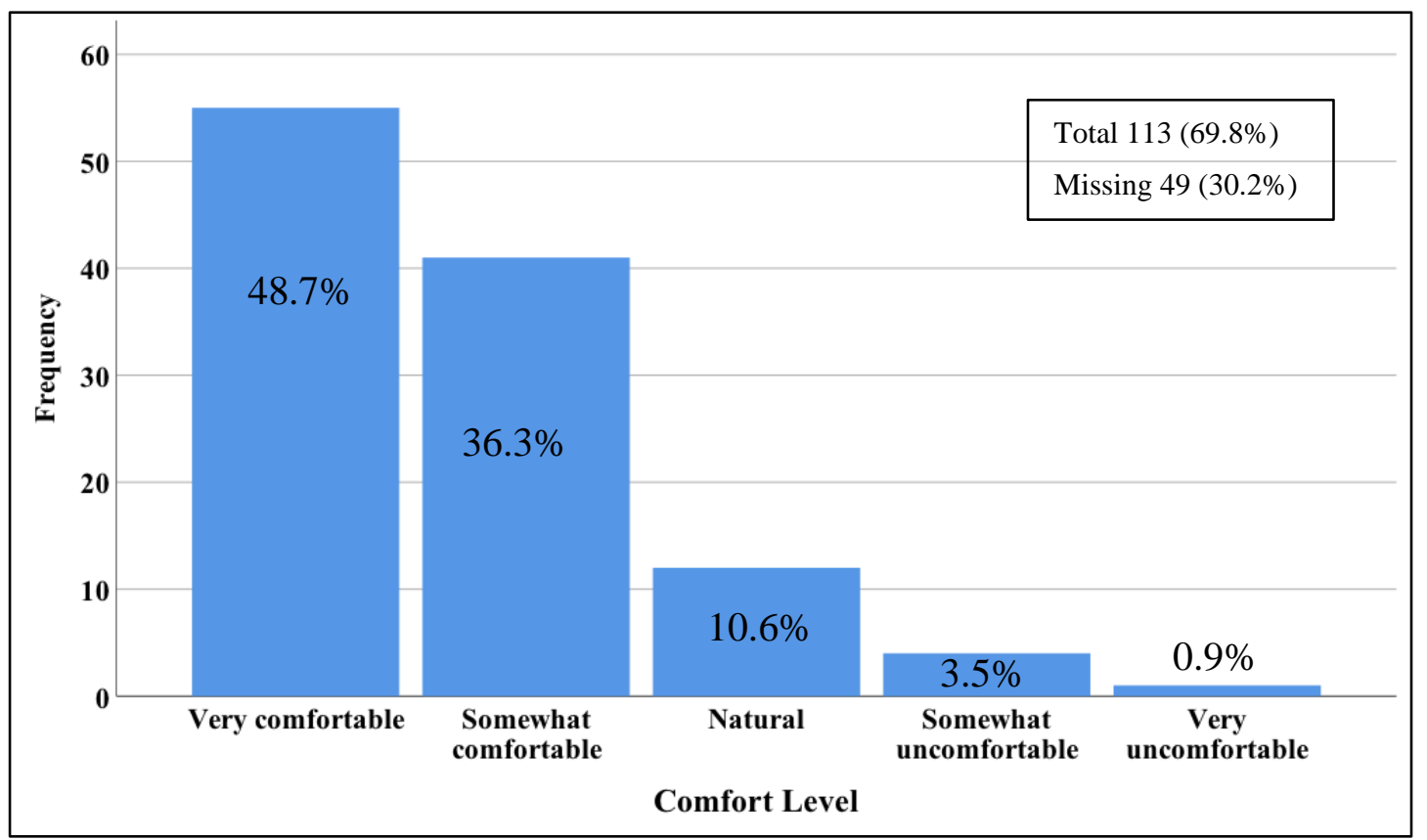

Figure 7-3: Parents/carers comfort with using a Tablet Computer

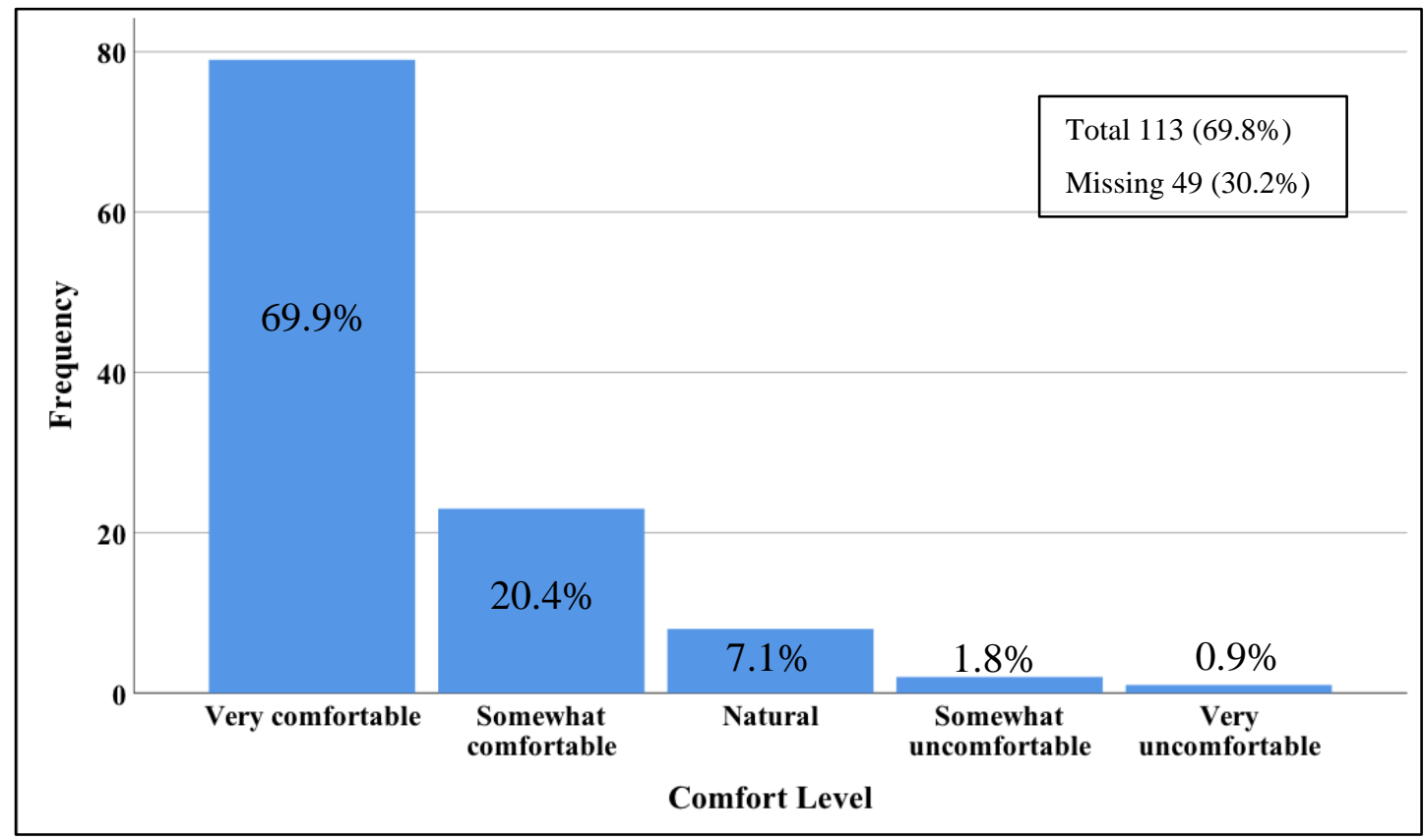

Figure 7-4: Parents/carers comfort with using a Mobile Phone/Smartphone 


\subsection{Parent/carer perceptions on NODA using UTAUT/added constructs}

Table 7-2 provides an overview of parent/carer perceptions on NODA using UTAUT/added constructs (performance expectancy, effort expectance, social influence, facilitating conditions, self-efficacy and anxiety). Over half of those surveyed answered these questions $(61.7 \%)$. The majority of participants answered "Agree" (combining strongly agree and agree) for all questions asked within each core construct, whilst most of them answered "Disagree" (combining disagree and strongly disagree) for all questions asked within the anxiety construct. In response to the question: 'If the NODA system had been available before my child's diagnosis, I would have used the NODA system?,' the overall response to this question was very positive. Most of those who answered indicated that that they "Agree" (66.0\%) (combining strongly agree and agree) while $20.0 \%$ of the participants reported "Disagree" (combining disagree and strongly disagree). Subsequently, the participants were asked a question "Why?" in order to identify the reasons behind their willingness or reluctance to use the NODA system for diagnostic assessment. The total number of participants who answered this question was 100 (61.7\%). As mentioned earlier in the methodology chapter, a thematic analysis was conducted for this openended question (See pages 147, 150). The main themes with regard to the potential reasons why parents/carers felt that they would either have used the NODA system, or not used the system, including the representative quotations as illustrative examples that cited by parents/carers, were as follow (see Figure 7-5 \& Figure 7-6): 


\subsubsection{Potential reasons of parents/carers who felt they would have used the NODA system:}

\section{a) Speedier diagnosis}

A number of parents/carers indicated that they would have used the NODA system if it had been available prior to their child's diagnosis, because it could help to receive an earlier diagnosis or even provided indication of a probable diagnosis:

"Because professionals could not see what we did until he was 10. Our son now has related mental health problems due to delayed diagnosis."

(Mother of a 12-year- old boy, diagnosed with Autism at the age of 10 years)

"Because it took too long to get a diagnosis."

(Mother of an 18-year- old girl, diagnosed with Autism at the age of 17 years)

"Quicker diagnosis."

(Mother of a 9-year- old boy, diagnosed with ASD at the age of 9 years)

"Would give a much quicker indication of a possible diagnosis."

(Mother of a 15-year- old boy, diagnosed with Asperger syndrome at the age of 14 years)

"Because it would have given us a chance if earlier help and may have meant he was supported and understood at school."

(Mother of a 17-year- old boy, diagnosed with ASD at the age of 14 years)

"Because my son would not have had to wait for over 2 years on a CAMHS waiting list before he received his first appointment."

(Mother of a 10-year- old boy, diagnosed with ASD at the age of 7 years)

"Professionals could have diagnosed earlier."

(Mother of a 11-year- old boy, diagnosed with Asperger syndrome at the age of 7 years)

"It took 3 years of living with violent aggressive behaviour before we had diagnosis, I would have tried anything that would have sped up the process." (Mother of a 13-year- old boy, diagnosed with Autism at the age of 12 years) 


\section{b) Ease of use}

Some parents/carers reported that they would have used the NODA system because it appeared to them easy to use:

"It seems straight forward so I would not be apprehensive about using it." (Mother of a 12-year- old boy, diagnosed with Asperger syndrome at the age of 12 years)

"Looks easy to use."

(Mother of a 16-year- old boy, diagnosed with ASD at the age of 4 years)

\section{c) Usefulness of NODA system if it is used in conjunction with face to face assessments}

Some participants highlighted that they would have used the NODA system because it is useful tool that would capture the behaviours as they occurred, but besides the face to face assessment:

"I think this would have been a useful tool in addition to seeing specialists in person for assessment, as it would capture real moments at home that often do not present themselves in the artificial and stressful professional environment. I think it would help the parents/carers feel their opinions are valid and real." (Mother of a 6-year- old boy, diagnosed with ASD at the age of 6 years)

"Although I feel it is useful... it is not enough to move to a diagnosis. Clinical human assessment with interaction is vital to diagnosis."

(Mother of a 11-year- old boy, diagnosed with Asperger syndrome at the age of 9 years)

"I think that when professionals visit the home to observe they only get a snapshot to base their assessment on. Using NODA would give a clearer picture as you can capture the behaviour as it happens. However, I think that NODA should be used in conjunction with face to face support and assessment and not separated."

(Mother of a 9-year- old boy, diagnosed with ASD at the age of 7 years)

\section{d) Facilitating the behavioural observation in a child's natural environment}

A number of participants reported that use of the NODA system might show a clearer picture of the child's behaviours in their natural environment, which may not be apparent in a professional setting:

"Professionals would get a clearer picture of the child in their own 
surroundings."

(Mother of a 9-year- old boy, diagnosed with ASD at the age of 9 years)

"I think in addition to hopefully reduction in wait times etc., assessments could be broken into manageable parts and carried out in the child's natural environment. This could provide a much clearer picture of the child's profile than an artificial environment."

(Mother of a 10-year- old girl, diagnosed with ASD at the age of 3 years)

"It may have help us convince professionals that our daughter's behaviour at home was not always the same as school."

(Mother of a 15-year- old girl, diagnosed with Autism at the age of 11 years)

"Because most behaviours only occur at home and not necessarily in a professional environment. The child cannot just 'act autistic' on que. Most of the time the most prominent traits occur when the child feels comfortable enough to express them. I.e., my son may keep something in that has been bothering him in school all day, but as soon as he gets home from school the comfort of home allows him to express his emotions in a way that only an autistic person knows how."

(Mother of a 7-year- old boy, diagnosed with Autism at the age of 3 years)

"Seeing a child in their normal environment is more useful than in strange place with strangers."

(Mother of an 18-year- old boy, diagnosed with Autism at the age of 13 years)

"I think this system would be so beneficial to families to be able to show a professional a video of how your child's behaviour and responses are affecting them daily."

(Mother of a 7-year- old girl, diagnosed with ASD at the age of 7 years)

"Because it shows the professionals what your child is like in real environment not in their waiting areas."

(Mother of a 7-year- old boy, diagnosed with Autism at the age of 3 years)

\section{e) Providing a useful record to evidence behaviours}

Several participants highlighted the fact that with a NODA system it could use the recorded videos as evidence and may also enable professionals to share this evidence amongst other professionals, both during and after diagnosis:

"We waited 5 years for diagnosis due to lack of evidence as my son was home schooled. His whole secondary education and years wasted. I would use this new system as a faster diagnostic tool and as evidence."

(Mother of a 17-year- old boy, diagnosed at the age of 17years) 
"Because its evidence."

(Mother of a 7-year- old boy, diagnosed with Autism at the age of 3 years)

"The depth of information collected would be improved and could be shared with the relevant professionals during and post diagnosis (e.g. occupational therapist, speech and language therapist etc.) to provide background and prevent needing constant reassessments etc."

(Mother of a 10-year- old girl, diagnosed with ASD at the age of 3 years)

"It might help to show behaviours and concerns to professionals so that they can build a better understanding. Also, professionals often do not listen to us as parents and so actual evidence would help get our point across."

(Father of an 18-year- old boy, diagnosed with Asperger syndrome at the age of 8 years)

"Seems like a very effective way of collecting the evidence needed of day to day life that can be overlooked by doctors basing their findings on assessments, in particular my second child my daughter that just fell below the average scoring for ASD based on the Autism Diagnostic Observation Schedule (ADOS) assessment when it's proven that this assessment is not as effective in females as it is males, showing evidence of behaviour in day to day life would be helpful."

(Mother of an 8-year- old daughter, diagnosed with ASD at the age of 5 years)

"It was sometimes difficult to explain behaviours during the diagnostic period. Having video would have made it easier to show what was happening rather than relying on verbal explanation."

(Mother of a 11-year- old boy, diagnosed with Autism at the age of 2 years)

“As part of my son's diagnosis I gave the paediatrician videos of his behaviour that concerned me as well as videos of how he used to be before his regression. These videos played a big part in his diagnosis."

(Mother of a 2-year- old boy, diagnosed with Autism at the age of 2 years)

"Having visual evidence speaks for itself, in fact I have videos of my son stimming."

(Mother of a 12-year- old boy, diagnosed with Autism at the age of 2 years)

"It is easier to see the behaviour/s with sight than to interpret behaviours in a written statement."

(Mother of a 12-year- old boy, diagnosed with Asperger syndrome at the age of 11 years) 
"I recorded my child's behaviour on my smart phone for my son psychologist and because of the video evidence she took more notice."

(Mother of a 12-year- old boy, diagnosed with ASD at the age of 8 years)

\section{f) Possibility of helping to receive early interventions}

Some parents/carers felt that they would have used the NODA system because they think it might have meant that their child could have accessed the early intervention services:

"If this had been available previously my sons would have had early intervention."

(Mother of a 12-year- old boy, diagnosed with ASD at the age of 11 years)

\section{g) Less stressful}

Some participants felt that diagnostic assessment conducted by using such system would be less stressful for parents and their children:

"Would have made it less stressful."

(Mother of a 6-year- old boy, diagnosed with ASD at the age of 4 years)

"It might have spared our family a lot of stress and pain."

(Mother of a 7-year- old boy, diagnosed with ASD at the age of 7 years) 


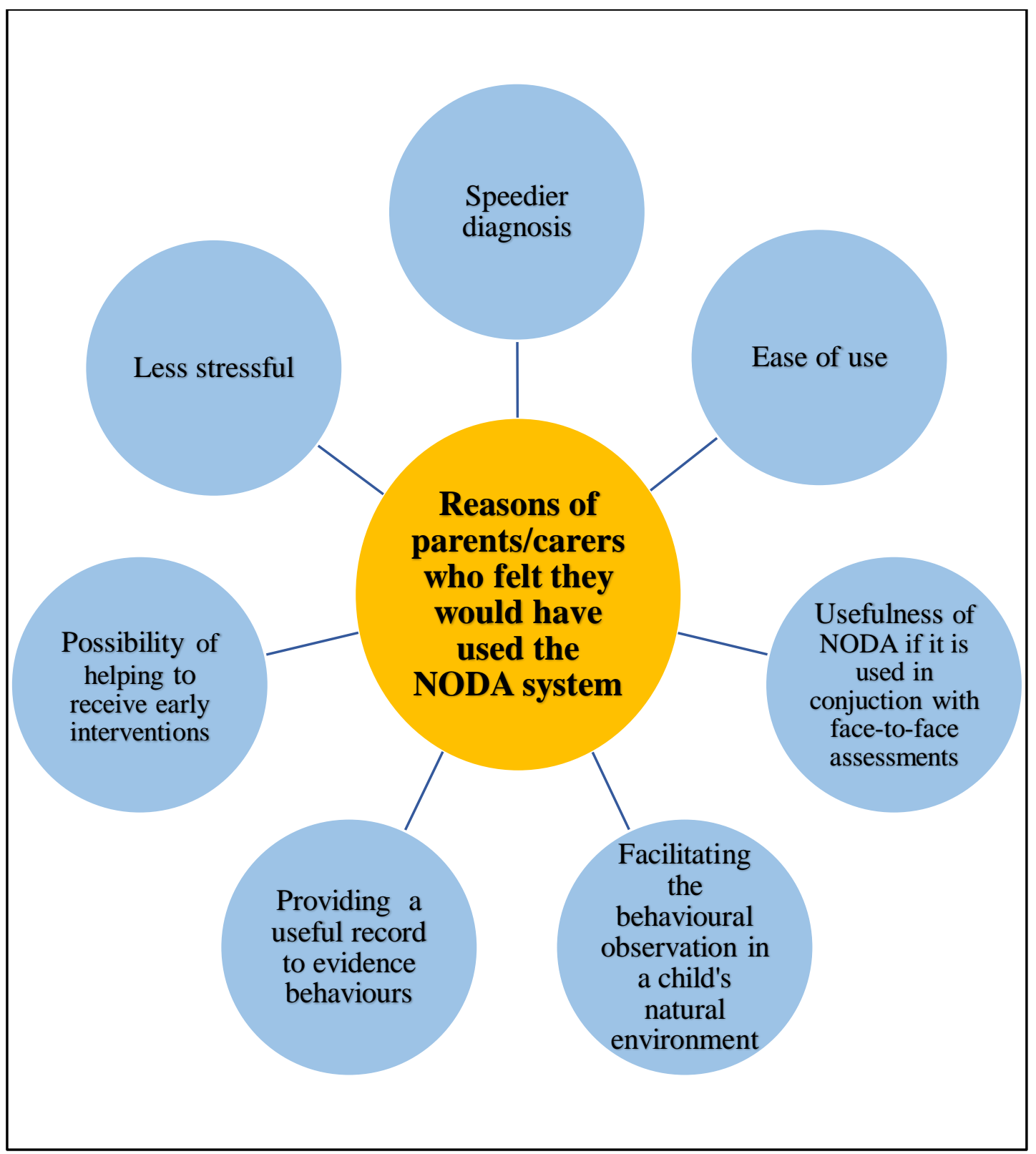

Figure 7-5: Potential reasons of parents/carers who felt they would have used the NODA system 


\subsubsection{Potential reasons of parents/carers who felt they would not have used the NODA system:}

\section{a) Lack of capturing complexity of issues}

The parents/carers expressed that they would not have used the NODA system because they thought that recorded videos may not be enough to provide a clear picture of the child's behaviours and may not capture all of issues:

"I do not think a small snapshot of a child's problems gives an accurate enough picture to get a thorough diagnosis containing helpful information on a child with specific difficulties."

(Mother of a 14-year- old boy, diagnosed with Asperger syndrome at the age of 11 years)

"I do not think few videos can capture all the issues that my child has."

(Mother of an 8-year- old boy, diagnosed with ASD at the age of 3 years)

\section{b) Resistance to being filmed and behaviour masking}

Some parents/carers reported that would not have used the NODA system as some children might not tolerate being recorded and some of them have the ability to mask their behaviours:

"Because my daughter hides a lot of her traits and is fully aware of masking when a phone is even in the room never mind recording her and if recording a meltdown, the phone would get broken. naturally behave. One of her abilities is incredible attention to detail so she would see a camera wherever I hid it!" (Mother of a 9-year- old girl, diagnosed with Asperger syndrome at the age of 9 years)

"I do not think there is any substitute for a child being observed in person by a clinician. Especially girls are able to mask behaviour and difficulties and need to be observed to read between the lines."

(Mother of a 9-year- old girl, diagnosed with Asperger syndrome at the age of 8 years)

"My child would not tolerate being recorded."

(Mother of a 17-year- old boy, diagnosed with Autism at the age of 2 years) 
"It can be very hard to film my child behaviours as he may act worse or may stop doing what he's doing as soon as he sees my phone. I also worry that I may upset him even more than he already was before I started filming."

(Mother of a 6-year- old boy, diagnosed with ASD at the age of 5 years)

\section{c) Inadequacy of relaying only on the NODA for diagnostic assessment}

Some parents/carers felt that depending solely on the NODA system for diagnostic assessment would be limited, although they thought that NODA would be a helpful tool if used along with in-person assessment. They also suggested that such a system could be a useful to be used as screening tool to determine if the child needs additional diagnostic assessments:

"The diagnosis process is full of uncertainty and needs a personal approach. Often parents are confused and trying to comprehend far too much information. I would also not like to see observations replaced by technology. By what means can one assure the information recorded is not a biased sample of the child's behaviour. I would however agree that this could compliment the process and work well alongside clinical observations."

(Mother of a 15-year- old boy, diagnosed with Autism at the age of 15 years)

"I think it would be a useful tool alongside appointments as not all behaviour and difficulties are observed during the appointment time. However, using this tool to solely diagnose may be difficult."

(Mother of a 12-year- old boy, diagnosed with Autism at the age of 4 years)

"If the diagnosis is relying only on these videos it is hugely limited. Autism is a lifelong diagnosis and a multitude of investigations is needed. I like the idea of natural home videos but not independent of other assessments."

(Mother of a 6-year- old boy, diagnosed with Autism at the age of 4 years)

"I can see the NODA system being used as a screening tool to see if a child needed further assessment but would be uncomfortable to see this being used as the main way to diagnose autism."

(Mother of a 9-year- old boy, diagnosed with Autism at the age of 7 years) 


\section{d) Possibility of parental recording bias}

The participants stated that they would not have used the NODA system as they might be biased while they are recording their children' behaviours:

"It would be a biased snap shot from the parent's perspective and therefore useless."

(Father of a 15-year- old boy, diagnosed with Asperger syndrome at the age of 5 years)

\section{e) Parent/carer preference of face to face interaction with professionals}

Some participants expressed the fact that they prefer face to face interaction with professionals, allowing the opportunity to discuss, ask enquiries and notice the professional's responses:

"I think I would have needed a more face to face interaction at the time of diagnosis. My son's father did not take the news well and for him in particular this would have seemed very fake. We needed to see the doctors for it to seem real."

(Mother of a 7-year- old boy, diagnosed with ASD at the age of 3 years)

"For something so serious, I would always want face to face in person interaction so that I can assess the professional's responses e.g. whether they are listening \& hearing or dismissive so I can decide whether I have trust in them. I would want to be able to raise questions with them, especially about implications of diagnosis, information about potential co-morbid conditions etc., again face to face in person."

(Mother of an 18-year- old girl, diagnosed with ASD at the age of 17 years)

"I needed to see and speak to the ASD assessment team because I was confused about the diverse symptoms, the overlapping medical conditions (DCD, joint hypermobility, $A D D)$ )."

(Mother of a 9-year- old boy, diagnosed with Autism at the age of 7 years)

\section{f) Privacy concerns}

Some respondents were concerned about potential privacy related issues involved with sending their children images or videos through the internet:

"I think it unlikely I could have captured the behaviours of concern on video, concerning to share the images via technology."

(Mother of an18-year- old boy, diagnosed with ASD at the age of 17 years) 


\section{g) Possibility of not suiting all cases}

The participants reported that they would not have used the NODA system because it may not be suitable for all children, as each child has a different case:

"I think the NODA system could work but not for everyone as some children act differently in different environments."

(Sister of an18-year- old boy, diagnosed at the age of 18 years)

"My son is described as complex by the professionals. I do not think that the NODA system would have been enough for him to be diagnosed."

(Mother of a 13-year- old boy, diagnosed with Autism at the age of 11 years)

"I know my children would not sit and play with toys. I also know if I tried to get him to sit it would cause further problem. I do like the idea but it would not suit my child at all."

(Mother of a 5-year- old boy, diagnosed with ASD at the age of 4 years)

\section{h) Uncertainty of using NODA in right way}

Some participants felt that they were not sure if they could use the NODA system correctly:

"In case I missed it up."

(Mother of a 2-year- old girl, diagnosed with ASD at the age of 2 years)

"I am unsure if I would be able to use it. What if I meet up all the saved data by hitting a wrong button."

(Mother of a 11-year- old girl, diagnosed with ASD at the age of 11 years)

In summary, parents/carers who felt they would use the NODA system, considered NODA to be an effective tool that could address some of the challenges related to ASD diagnosis. They felt that they would have used the NODA system in order to speed up the diagnostic process and receive either early diagnosis or indication of a possible diagnosis. This is presumably because the majority of participants were dissatisfied with the time taken to obtain a diagnosis, given that a significant time generally elapsed (Mean $=46$ months) between seeking help and receiving a confirmed diagnosis (See Chapter 6). They highlighted that due to delayed diagnosis, some children developed mental health problems and others had violent aggressive 
behaviours. They emphasised that early diagnosis could allow children to access early intervention services and be better understood at school.

Furthermore, the participants drew attention to the fact that NODA can allow professionals to observe the child's behaviour in a natural environment as well as enabling parents/carers to provide evidence of their child's behaviour. They reported that most of the behaviours only occur at home, when the child feels comfortable enough to express them, and this may not be accessible in a professional environment in the presence of a professionals. In addition, some children are home schooled and due to a lack of evidence they had to wait a very long time (e.g. 5 years) to receive the diagnosis. They also emphasised that explaining behaviours to the professionals might be difficult, so having video evidence would make it easier to show what was actually happening, instead of relying on a verbal explanation or written statements. They suggested that video evidence might be used even after receiving a diagnosis, as it could be shared with other health professionals such as occupational and speech and language therapists. They also indicated that using such a system to conduct a diagnosis would be less stressful for the children and their families.

With regard to those parents/carers who felt they would not have used the NODA system, there were several reasons why they felt that NODA might not be an appropriate tool for remote ASD diagnostic assessment. Their various text responses indicated that some children are able to mask their behaviours and may need to be observed by in-person approach. They felt that some children would not tolerate being recorded on a camera phone and would be able to notice it, wherever they might have hidden it. On noticing the phone their behaviour could worsen, or they might stop the recording altogether. Furthermore, they thought that recorded videos taken by parents/carers may not be useful and correct in order to make a diagnosis by clinicians. Other parents/carers were concerned about the privacy issues that related to sending videos via technology. Other parents/carers expressed their preference to have face to face interaction with professionals in order to notice their responses, discuss and ask enquiries e.g. about diverse symptoms, overlapping medical conditions, and comorbidities. Some parents also anticipated that NODA could not capture all of the issues and may not be appropriate for all children, particularly those who have mild conditions. Interestingly, it has been suggested by both parents/carers who felt they 
would and would not have used the NODA system, that NODA would be more useful if it could be used alongside the face to face approach instead of replacing it.

Overall, some parents/carers who felt they would use NODA system considered such a system useful for providing remote diagnostic assessment of ASD. However, the parents/carers who felt they would not use NODA system thought that NODA might not be appropriate way of conducting the diagnostic assessment remotely. Hence, NODA has the potential to be used for ASD diagnosis and assessment, but may not be sufficient for those children with mild autism symptoms. Comprehensive in-person assessments could be essential for them, although the NODA system could be used in such cases as a screening tool, to improve the diagnostic process as whole. 


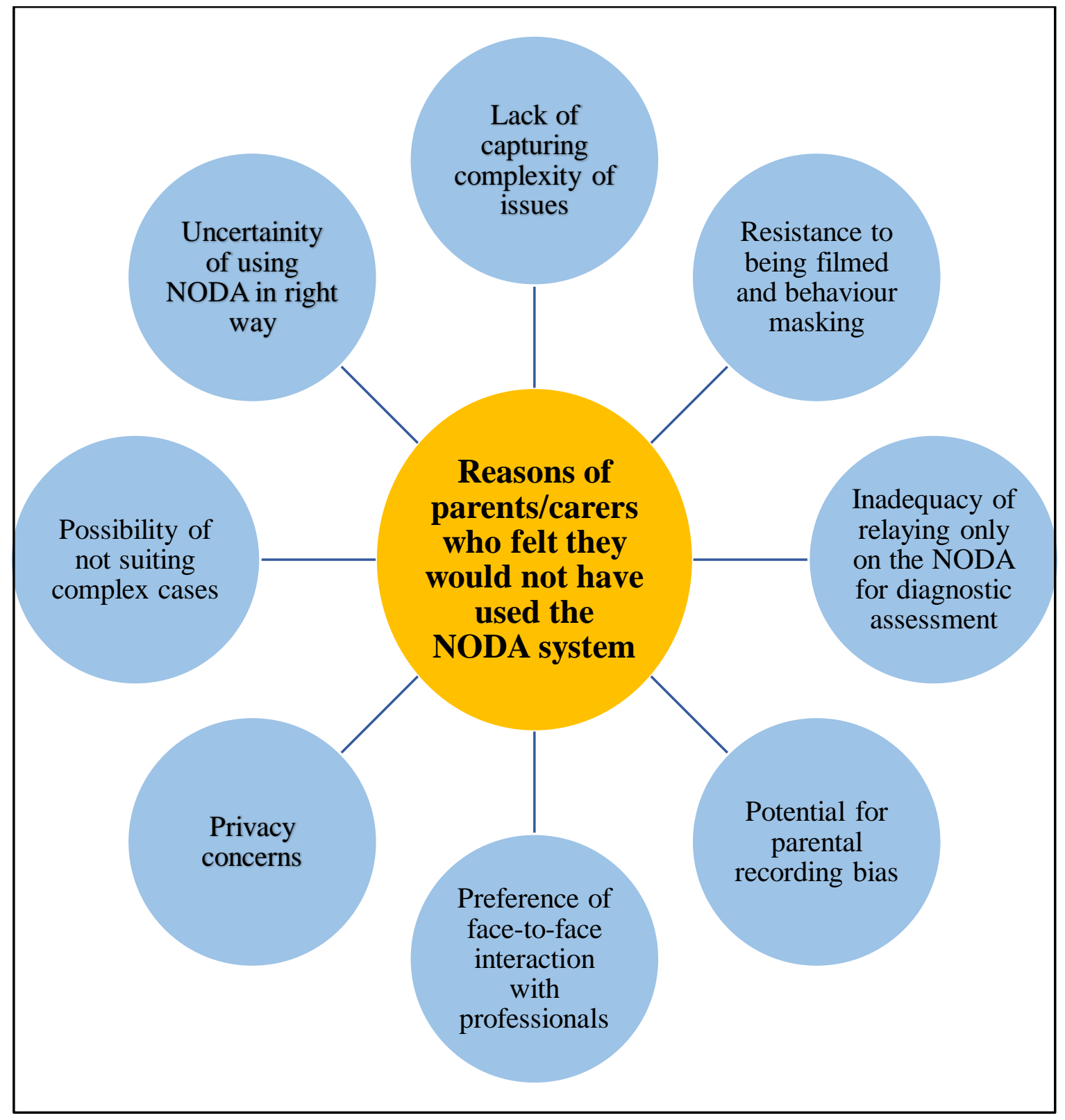

Figure 7-6: Potential reasons of parents/carers who felt they would not have used the NODA system 
Table 7-2: Perceptions of parents/carers on NODA using UTAUT/added constructs

\begin{tabular}{|c|c|c|c|c|c|c|}
\hline \multirow{2}{*}{$\begin{array}{l}\text { Questions asked } \\
\text { Performance Expectancy }\end{array}$} & \multicolumn{6}{|c|}{ Frequency (\%) } \\
\hline & $\begin{array}{c}\text { Strongly } \\
\text { Agree }\end{array}$ & Agree & Disagree & $\begin{array}{l}\text { Strongly } \\
\text { Disagree }\end{array}$ & Don't know & Total/Missing \\
\hline $\begin{array}{l}\text { I would find the NODA } \\
\text { system useful in the diagnosis } \\
\text { of my child. }\end{array}$ & $20(20 \%)$ & $52(52 \%)$ & $13(8.0 \%)$ & $5(5.0 \%)$ & $10(10.0 \%)$ & $\begin{array}{l}100(61.7 \%) \\
62(38.3 \%) \\
\end{array}$ \\
\hline $\begin{array}{l}\text { Using the NODA system } \\
\text { would enable me to receive } \\
\text { the diagnosis quickly. }\end{array}$ & $17(17.0 \%)$ & $41(41.0 \%)$ & $14(14.0 \%)$ & $4(4.0 \%)$ & $24(24.0 \%)$ & $\begin{array}{l}100(61.7 \%) \\
62(38.3 \%)\end{array}$ \\
\hline $\begin{array}{l}\text { Using the NODA system to } \\
\text { obtain a diagnosis, for my } \\
\text { child would be productive in } \\
\text { accessing services. }\end{array}$ & $13(13.0 \%)$ & $44(44.0 \%)$ & $15(15.0 \%)$ & $8(8.0 \%)$ & $20(20.0 \%)$ & $\begin{array}{c}100(61.7 \%) \\
62(38.3 \%)\end{array}$ \\
\hline $\begin{array}{l}\text { If I use the NODA system, it } \\
\text { would increase chances of } \\
\text { getting an earlier diagnosis } \\
\text { for my child }\end{array}$ & $19(19.0 \%)$ & $42(42.0 \%)$ & $16(16.0 \%)$ & $5(5.0 \%)$ & $18(18.0 \%)$ & $\begin{array}{c}100(61.7 \%) \\
62(38.3 \%)\end{array}$ \\
\hline Effort Expectancy & $\begin{array}{l}\text { Strongly } \\
\text { Agree }\end{array}$ & Agree & Disagree & $\begin{array}{l}\text { Strongly } \\
\text { Disagree }\end{array}$ & Don't know & Total/Missing \\
\hline $\begin{array}{l}\text { From watching the video, I } \\
\text { think I would have clear } \\
\text { understanding of how I would } \\
\text { interact with the NODA } \\
\text { system. }\end{array}$ & $21(21.0 \%)$ & $62(62.0 \%)$ & $7(7.0 \%)$ & $3(3.0 \%)$ & $7(7.0 \%)$ & $\begin{array}{c}100(61.7 \%) \\
62(38.3 \%)\end{array}$ \\
\hline $\begin{array}{l}\text { If I have training, I think it } \\
\text { would be easy for me to } \\
\text { become skillful at using the } \\
\text { NODA system. }\end{array}$ & $21(21.0 \%)$ & $67(67.0 \%)$ & $4(4.0 \%)$ & $2(2.0 \%)$ & $6(6.0 \%)$ & $\begin{array}{c}100(61.7 \%) \\
62(38.3 \%)\end{array}$ \\
\hline
\end{tabular}




\begin{tabular}{|c|c|c|c|c|c|c|}
\hline $\begin{array}{l}\text { I think I would find the } \\
\text { NODA system easy to use. }\end{array}$ & $20(20.0 \%)$ & $64(64.0 \%)$ & $4(4.0 \%)$ & $3(3.0 \%)$ & $9(9.0 \%)$ & $\begin{array}{c}100(61.7 \%) \\
62(38.3 \%) \\
\end{array}$ \\
\hline $\begin{array}{l}\text { I think learning how to use } \\
\text { the NODA system would be } \\
\text { easy for me. }\end{array}$ & $25(25.0 \%)$ & $64(64.0 \%)$ & $3(3.0 \%)$ & $1(1.0 \%)$ & $7(7.0 \%)$ & $\begin{array}{l}100(61.7 \%) \\
62(38.3 \%)\end{array}$ \\
\hline Social Influence & $\begin{array}{l}\text { Strongly } \\
\text { Agree }\end{array}$ & Agree & Disagree & $\begin{array}{l}\text { Strongly } \\
\text { Disagree }\end{array}$ & Don't know & Total/Missing \\
\hline $\begin{array}{l}\text { My family members would } \\
\text { think that I should use the } \\
\text { NODA system }\end{array}$ & $9(9.0 \%)$ & $33(33.0 \%)$ & $19(19.0 \%)$ & $10(10.0 \%)$ & $29(29.0 \%)$ & $\begin{array}{l}100(61.7 \%) \\
62(38.3 \%)\end{array}$ \\
\hline $\begin{array}{l}\text { My friends would think that I } \\
\text { should use the NODA system }\end{array}$ & $8(8.0 \%)$ & $35(35.0 \%)$ & $15(15.0 \%)$ & $9(9.0 \%)$ & $33(33.0 \%)$ & $\begin{array}{c}100(61.7 \%) \\
62(38.3 \%) \\
\end{array}$ \\
\hline $\begin{array}{l}\text { I would like to think that } \\
\text { professionals would support } \\
\text { the use of the NODA system } \\
\text { (e.g. GP, physiologists, health } \\
\text { professionals, teachers) }\end{array}$ & $17(17.0 \%)$ & $55(55.0 \%)$ & $7(7.0 \%)$ & $8(8.0 \%)$ & $13(13.0 \%)$ & $\begin{array}{l}100(61.7 \%) \\
62(38.3 \%)\end{array}$ \\
\hline Facilitating conditions & $\begin{array}{l}\text { Strongly } \\
\text { Agree }\end{array}$ & Agree & Disagree & $\begin{array}{l}\text { Strongly } \\
\text { Disagree }\end{array}$ & Don't know & Total/Missing \\
\hline $\begin{array}{l}\text { I would have the resources } \\
\text { necessary to use the NODA } \\
\text { system (e.g. internet, } \\
\text { computer, smart phone, iPad) }\end{array}$ & $36(36.0 \%)$ & $56(56.0 \%)$ & $6(6.0 \%)$ & $1(1.0 \%)$ & $1(1.0 \%)$ & $\begin{array}{l}100(61.7 \%) \\
62(38.3 \%)\end{array}$ \\
\hline $\begin{array}{l}\text { I would have the knowledge } \\
\text { necessary to use the NODA } \\
\text { system }\end{array}$ & $25(25.0 \%)$ & $62(62.0 \%)$ & $3(3.0 \%)$ & $1(1.0 \%)$ & $9(9.0 \%)$ & $\begin{array}{c}100(61.7 \%) \\
62(38.3 \%)\end{array}$ \\
\hline
\end{tabular}




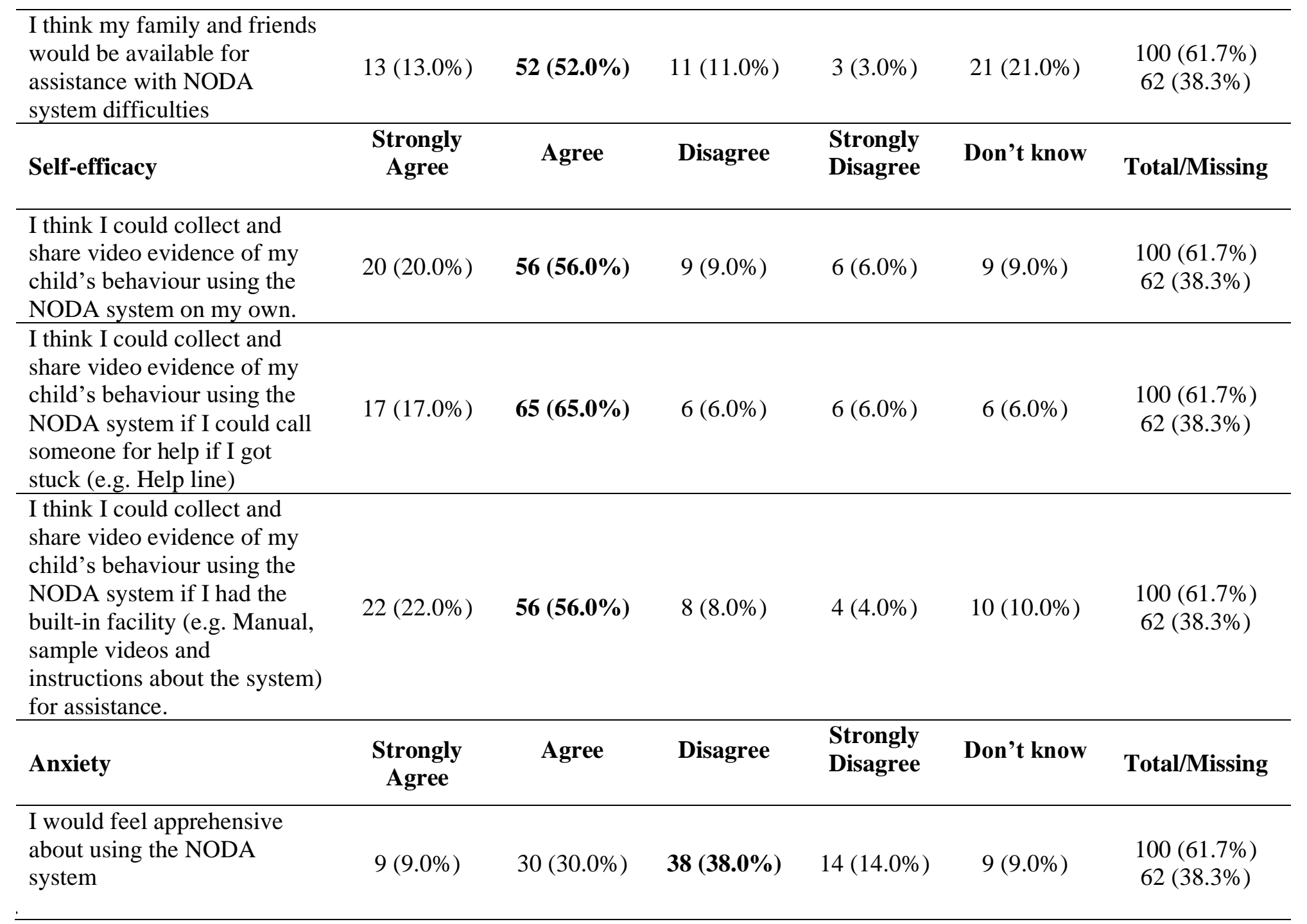




\begin{tabular}{|c|c|c|c|c|c|c|}
\hline $\begin{array}{l}\text { It would scare me to think } \\
\text { that I could lose a lot of } \\
\text { information using the NODA } \\
\text { system by hitting the wrong } \\
\text { key }\end{array}$ & $6(6.0 \%)$ & $31(31.0 \%)$ & $41(41.0 \%)$ & $12(12.0 \%)$ & $10(10.0 \%)$ & $\begin{array}{l}100(61.7 \%) \\
62(38.3 \%)\end{array}$ \\
\hline $\begin{array}{l}\text { I would hesitate to use the } \\
\text { NODA system for fear of } \\
\text { making mistakes I cannot } \\
\text { correct }\end{array}$ & $6(6.0 \%)$ & $23(23.0 \%)$ & $41(41.0 \%)$ & $17(17.0 \%)$ & $13(13.0 \%)$ & $\begin{array}{l}100(61.7 \%) \\
62(38.3 \%)\end{array}$ \\
\hline $\begin{array}{l}\text { The NODA System would be } \\
\text { somewhat intimidating to me }\end{array}$ & $1(1.0)$ & $16(16.0 \%)$ & $48(48.0 \%)$ & $24(24.0 \%)$ & $11(11.0 \%)$ & $\begin{array}{c}100(61.7 \%) \\
62(38.3 \%) \\
\end{array}$ \\
\hline Behavioural Intention & $\begin{array}{l}\text { Strongly } \\
\text { Agree }\end{array}$ & Agree & Disagree & $\begin{array}{l}\text { Strongly } \\
\text { Disagree }\end{array}$ & Don't know & Total/Missing \\
\hline
\end{tabular}




\subsection{Parents/carers anticipated usage of NODA}

Table 7-3 presents the anticipated usage of NODA by parents/carers. In response to the question: 'Would you have considered using the NODA system to receive a diagnosis for your child if it had been offered free by the NHS?' $67 \%$ of those who responded, reported "Yes". When the participants were asked what they thought about using the NODA system if it had been offered privately, and accepted by the NHS as an official diagnosis (the price would be around $£ 180$ ), $41.4 \%$ of those who responded, reported "Yes". This might indicate that parents/carers of children with ASD are frustrated about the current diagnostic services that are provided in Wales. Furthermore, it appeared that they prefer to wait for the diagnostic process to be improved by the NHS, as that would mean that services would not have to be provided and received by different healthcare systems (NHS/Private).

Table 7-3: Expected usage of NODA

\begin{tabular}{ll}
\hline $\begin{array}{l}\text { Anticipated usage of NODA if it had } \\
\text { been offered free by the NHS, } \mathbf{n}(\boldsymbol{\%})\end{array}$ \\
Yes & $67(67 \%)$ \\
No & $17(17.0 \%)$ \\
I don't know & $16(16.0 \%)$ \\
Total & $100(61.7 \%)$ \\
Missing & $62(38.3 \%)$ \\
\hline Anticipated usage of NODA if it had & \\
been offered privately and accepted by & \\
the NHS as an official diagnosis, (the & \\
price would be around $£ 180), \mathbf{n}(\boldsymbol{\%})$ & \\
Yes & $41(41.4 \%)$ \\
No & $26(26.3 \%)$ \\
I don't know & $32(32.3 \%)$ \\
Total & $99(61.1 \%)$ \\
Missing & $63(38.9 \%)$ \\
\hline
\end{tabular}




\subsection{Reliability of the constructs}

We performed an internal consistency reliability analysis using Chronbach's Alpha for the subscales: performance expectancy, effort expectancy, social influence, facilitating condition, self-efficacy and anxiety (see Table 7-4). It was evaluated using the guidelines suggested by Hinton, McMurray, \& Brownlow (2004); an Alpha score above .75 is generally considered to show a scale of high reliability, 0.5 to 0.75 is acceptable and taken to indicate a scale of a moderate reliability, while below this shows a low reliable scale (Hinton et al., 2004). All of the Likert scales produced reliable results ( 0.670 or higher).

Table 7-4: Reliability of the constructs

\begin{tabular}{lcc}
\hline \multicolumn{1}{c}{ Model construct } & $\begin{array}{c}\text { Cronbach's } \\
\text { Alpha }\end{array}$ & $\begin{array}{c}\text { Number of } \\
\text { Items }\end{array}$ \\
\hline Performance Expectancy & 0.853 & 4 \\
Effort Expectancy & 0.845 & 4 \\
Social Influence & 0.779 & 3 \\
Facilitating Conditions & 0.670 & 3 \\
Self-Efficacy & 0.839 & 3 \\
Anxiety & 0.731 & 4 \\
\hline
\end{tabular}

\subsection{Univariate analysis of the association of variables on behavioural intention of using NODA}

In this univariate analysis, the significant factors that were associated with the behavioural intention to use NODA for diagnostic assessment were mainly related to the variables of the original UTAUT model. These significant factors were performance expectancy (mean 2.78 (not negative intention) vs. 1.59 (negative intention); difference $1.18 ; 95 \%$ CI $[.79,1.58])$; this means that if the parents/carers of children with ASD believe that using the NODA system would reduce the time taken to obtain a diagnosis, they would be more motivated to use the NODA system, effort expectancy (mean 3.14 (not negative intention) vs. 2.47 (negative intention); difference .67; 95\% CI: $[.34, .99])$; this implies that if parents/carers believe that interaction with the NODA system was clear, easy to use and understandable, it might 
improve their behavioural intention to accept the system, social influence (mean 2.42 (not negative intention) vs. 1.31 (negative intention); difference 1.11; 95\% CI [.69, 1.53]); this indicates that if parents/carers believe that people who are important to them (e.g. family members, friends, professionals) recommend using the NODA system, they would be more likely to use it, facilitating conditions (mean 3.02 (not negative intention) vs. 2.49 (negative intention); difference .52; 95\% CI [.19, .86]); this implies that if parents/carers believe that the assistance, knowledge and resources necessary to use the NODA system are available to them (e.g. Internet, computer, smartphone/mobile phone) they would be more likely to use it, and self-efficacy (mean 3.04 (not negative intention) vs. 2.24 (negative intention); difference .80; 95\% CI [.43, 1.17]); this suggests that if parents/carers believe that they are able use to the NODA system, collect and share video evidence of their children on their own, they might be more motivated to use it.

However, no statistically significant associations were found between behavioural intention and anxiety, parent/carer demographics, experience with technology (internet/computer skills, comfort with using a desktop computer, laptop computer, tablet computer and mobile phone/smartphone), and the diagnostic process of ASD related variables, including satisfaction with the overall diagnostic process and the time taken to obtain a diagnosis of ASD (see Table 7-5). 
Table 7-5:Univariate analysis of the association of variables on Behavioural Intention of using NODA

\begin{tabular}{|c|c|c|c|c|}
\hline \multirow{2}{*}{ Variables } & \multicolumn{2}{|c|}{$\begin{array}{c}\text { Behavioural Intention to } \\
\text { use the NODA }\end{array}$} & \multirow{2}{*}{$\begin{array}{c}\text { Mean } \\
\text { Difference/ } \\
\text { Proportion }\end{array}$} & \multirow{2}{*}{$95 \% \mathrm{CI}$} \\
\hline & $\begin{array}{l}\text { Not Negative } \\
\text { Intention }\end{array}$ & $\begin{array}{l}\text { Negative } \\
\text { Intention }\end{array}$ & & \\
\hline $\begin{array}{l}\text { Parent's/carer's age, } \\
M(S D)\end{array}$ & $\begin{array}{l}\mathrm{N}=66 \\
39.56 \\
(7.69)\end{array}$ & $\begin{array}{l}\mathrm{N}=34 \\
39.44 \\
(9.99)\end{array}$ & 0.119 & $(-3.45$ to 3.69$)$ \\
\hline $\begin{array}{l}\text { Parent's/carer's } \\
\text { gender } \\
\text { Female, } \mathrm{n}(\%)\end{array}$ & $\begin{array}{c}\mathrm{N}=66 \\
63(95.45 \%)\end{array}$ & $\begin{array}{c}\mathrm{N}=34 \\
32(94.12 \%)\end{array}$ & $1.34 \%$ & $(-8 \%$ to $15 \%)$ \\
\hline $\begin{array}{l}\text { Parent's/carer's } \\
\text { educational } \\
\text { Higher education, n } \\
(\%) \\
\end{array}$ & $\begin{array}{c}\mathrm{N}=66 \\
30(45.45 \%)\end{array}$ & $\begin{array}{c}\mathrm{N}=34 \\
17(50 \%)\end{array}$ & $4.55 \%$ & $(-15 \%$ to $24 \%)$ \\
\hline $\begin{array}{l}\text { Marital Status } \\
\text { Cohabited/married, n } \\
(\%)\end{array}$ & $\begin{array}{c}\mathrm{N}=66 \\
54(81.82 \%)\end{array}$ & $\begin{array}{c}\mathrm{N}=34 \\
25(73.53 \%)\end{array}$ & $8.29 \%$ & $(-8 \%$ to $27 \%)$ \\
\hline $\begin{array}{l}\text { Computer/internet } \\
\text { skills } \\
\text { Average, advance, and } \\
\text { expert, } \mathrm{n}(\%)\end{array}$ & $56(84.85 \%)$ & $\begin{array}{c}\mathrm{N}=34 \\
27(79.41 \%)\end{array}$ & $5.44 \%$ & $(-9 \%$ to $23 \%)$ \\
\hline $\begin{array}{l}\text { Comfort with using } \\
\text { Desktop Computer } \\
\text { Comfortable, n }(\%)\end{array}$ & $\begin{array}{c}\mathrm{N}=66 \\
52(78.79 \%)\end{array}$ & $\begin{array}{c}\mathrm{N}=33 \\
27(81.82 \%)\end{array}$ & $3.03 \%$ & $(-15 \%$ to $18 \%)$ \\
\hline $\begin{array}{l}\text { Comfort with using } \\
\text { Laptop computer } \\
\text { Comfortable, } \mathrm{n}(\%)\end{array}$ & $\begin{array}{c}\mathrm{N}=66 \\
56(84.85 \%)\end{array}$ & $\begin{array}{c}\mathrm{N}=34 \\
29(85.29 \%)\end{array}$ & $0.44 \%$ & $(-16 \%$ to $14 \%)$ \\
\hline $\begin{array}{l}\text { Comfort with using } \\
\text { Tablet } \\
\text { Comfortable, } \mathrm{n}(\%)\end{array}$ & $\begin{array}{c}\mathrm{N}=66 \\
58(87.88 \%)\end{array}$ & $\begin{array}{c}\mathrm{N}=34 \\
28(82.35 \%)\end{array}$ & $5.53 \%$ & $(-8 \%$ to $22 \%)$ \\
\hline $\begin{array}{l}\text { Comfort with using } \\
\text { Mobile } \\
\text { Phone/Smartphone } \\
\text { Comfortable, n (\%) } \\
\end{array}$ & $\begin{array}{c}\mathrm{N}=66 \\
62(93.94 \%)\end{array}$ & $\begin{array}{c}\mathrm{N}=34 \\
29(85.29 \%)\end{array}$ & $8.65 \%$ & $(-3 \%$ to $25 \%)$ \\
\hline $\begin{array}{l}\text { Performance } \\
\text { Expectancy, } M(S D)\end{array}$ & $\begin{array}{c}\mathrm{N}=66 \\
2.78 \\
(1.00) \\
\end{array}$ & $\begin{array}{c}\mathrm{N}=34 \\
1.59 \\
(.84) \\
\end{array}$ & $1.18 *$ & (.79 to 1.58$)$ \\
\hline $\begin{array}{l}\text { Effort Expectancy, } \\
M(S D)\end{array}$ & $\begin{array}{l}\mathrm{N}=66 \\
3.14 \\
(.74) \\
\end{array}$ & $\begin{array}{l}\mathrm{N}=34 \\
2.47 \\
(.82) \\
\end{array}$ & $.67 *$ & (.34 to .99) \\
\hline $\begin{array}{l}\text { Social Influence, } \\
M(S D)\end{array}$ & $\begin{array}{c}N=66 \\
2.42 \\
(1.13) \\
\end{array}$ & $\begin{array}{l}\mathrm{N}=34 \\
1.13 \\
(.71) \\
\end{array}$ & $1.11 *$ & (.69 to 1.53$)$ \\
\hline $\begin{array}{l}\text { Facilitating } \\
\text { Conditions, } M(S D)\end{array}$ & $\begin{array}{l}N=66 \\
3.02 \\
(.76)\end{array}$ & $\begin{array}{l}N=34 \\
2.49 \\
(.87)\end{array}$ & $.52 *$ & (.19 to .86$)$ \\
\hline
\end{tabular}




\begin{tabular}{lccccc}
\hline Self-Efficacy, $M(S D)$ & $\mathbf{N}=66$ & $\mathbf{N}=34$ & & \\
& $\mathbf{3 . 0 4}$ & $\mathbf{2 . 2 4}$ & $\mathbf{. 8 0}$ & $\mathbf{( . 4 3}$ to 1.17) \\
& $\mathbf{( . 8 3 )}$ & $\mathbf{( . 9 5 )}$ & & \\
\hline Anxiety, $M(S D)$ & $\mathrm{N}=66$ & $\mathrm{~N}=34$ & & \\
& 1.93 & 2.07 & -.15 & $(-.46$ to .17$)$ \\
& $(.65)$ & $(.95)$ & & \\
\hline
\end{tabular}

Note. All of construct's items (performance expectancy, effort expectancy, social influence, facilitating conditions, self-efficacy and anxiety) were measured using Likert-Scale and recorded into: 4= Strongly Agree, 3= Agree, 2= Disagree, 1= Strongly Disagree 0= Don't Know, while the anxiety' items were recorded into: $1=$ Strongly Agree, $2=$ Agree, 3= Disagree, 4= Strongly Disagree 0= Don't. The mean score was then calculated for each construct's items to be used in the analysis. *Significant difference between groups with $p$ value $<0.05$

$M=$ Mean $; S D=$ Standard Deviation $; \mathrm{CI}=$ confidence interval

\subsection{Determination of predictors of parent/carer intention to use the NODA for diagnostic assessment in Wales}

A binary logistic regression analysis was used to examine which factors predicted the intention of parents/carers in Wales to use NODA for diagnostic assessment. The least significant variable that does not meet the level for staying in the model is removed. Once the variable is removed from the model, it remains excluded. The process is repeated until no other variables in the model meet the specified level for removal (Bursac et al., 2008; Pallant, 2016). The binary logistic regression was carried out two twice, once with missing data and once with imputed data. Behavioural intention to use the NODA was used as a dependent variable and it was dichotomised to not negative intention (combining strongly agree, agree, I don't know) and negative intention (combining strongly disagree, disagree). The examined variables are shown in Table 7-6. As we stated in previous sections, all of the items of the constructs (performance expectancy, effort expectancy, social influence, facilitating conditions, self-efficacy and anxiety) were measured using Likert-Scale and recorded into: 4= Strongly Agree, 3= Agree, 2= Disagree, 1= Strongly Disagree 0= Don't Know, while the items giving rise to anxiety were recorded into: $1=$ Strongly Agree, $2=$ Agree, $3=$ Disagree, $4=$ Strongly Disagree $0=$ Don't Know. The mean score was calculated for the items of each construct to be used in the analysis. In addition, in respect of the categorical predictors, some categories were collapsed, as there was a limited number of responses in some categories (Pallant, 2016). Before conducting the binary logistic regression, the assumptions of the logistic regression analysis (Pallant, 2016; Stoltzfus, 2011) were tested. These included: 
1. A dichotomous dependent variable.

The variable behavioural intention to use the NODA system was recorded into a dichotomous variable: not negative intention (combining strongly agree, agree, I don't know) and negative intention (combining strongly disagree, disagree). Having a "I don't know" option would also imply that those participants had a neutral/not negative opinion of using the NODA system for remote diagnostic assessment, thus this group have not been added to the negative intention category.

2. Absence of multicollinearity.

The absence of multicollinearity is the assumption that the predictor variables are not highly correlated, and was assessed using Tolerance values. Tolerance values that are very low (less than .1) indicate that the variable has high correlations with other variables in the model (see Page 127). None of the predictor variables was less than .1; therefore, we have not violated the multicollinearity assumption.

3. Absence of outliers

Although there was some indication of outlying cases (identified by Casewise List) (Pallant, 2016) (see Page 127), these were checked and investigated and nothing abnormal was found in the outlier values. Furthermore, exclusion of these cases did not affect the results. Therefore, these cases were kept, as outlier data are not always errors (Rousseeuw \& Hubert, 2018). 


\subsubsection{Behavioural intention of parents/carers to use the NODA model (with missing data)}

Using the binary logistic regression model, characteristics differentiating parents/carers who felt they would have used NODA, compared to those who felt they would not, were investigated (see Table 7-6). The age of the parent/carer, performance expectancy, social influence, comfort with using a laptop computer and comfort with using mobile phone/smartphone were incorporated into the model for behavioural intention to use NODA. Omnibus Tests of Model Coefficients was used, including Chi-Square, Degrees of Freedom (df) and p-value to check that the new model is an improvement over the baseline model. The purpose of the p-value in this test is to determine if the overall model is statistically significant. The results showed that the full model containing the five predictors was statically significant, [ChiSquare $=48.499, d f=5$ and $p=0.000(<0.05)]$, indicating that the model was able to distinguish between those parents/carers who reported a negative behavioural intention to use the NODA system and those who did not. All of the five predictors were significant at the $5 \%$ level.

Parent/carer age. Odds Ratio are the chance that a person who is older will be more likely to report a positive intention to use the NODA system. For every additional year on the age of parents/carers above the baseline, the chance that the person will report a positive intention increased by 1.10 times (e.g. baseline x $1.10 \mathrm{x}$ number of years).

Performance Expectancy. Odds Ratio are the chance that a person who has expectations of the NODA system's ability to reduce the time taken to obtain a diagnosis will be report a positive intention. For every additional degree of expectations above the baseline, the chance that the parent/carer will report a positive intention increased by 3.73 times.

Social Influence. Odds Ratio are the chance that a person who has belief that the people important to his/her (e.g. family members, friends, professionals) would recommend using NODA will be report a positive intention. For every additional degree of belief above the baseline, the chance that the parent/carer will report a positive intention increased by 2.78 times. 
Comfort with using Laptop Computer. Odds Ratio are the chance that parents/carers who were comfortable with using a laptop computer will more likely to report that they would have used the NODA system. For every additional degree of comfort above the baseline, the chance that the parent/carer will report a positive intention increased by 6.93 times.

Comfort with using Mobile phone/Smartphone. The strongest predictor of reporting a behavioural intention was comfort with using a mobile phone/smartphone. Odds Ratio are the chance that parents/carers who were comfortable with using a mobile phone/smartphone will more likely to report that they would have used the NODA system. For every additional degree of comfort above the baseline, the chance that the parent/carer will report a positive intention increased by 13.32 times.

The model correctly predicted $87.9 \%$ of cases where there was a behavioural intention to use the NODA system and $64.7 \%$ where there was none, giving an overall percentage correct prediction rate of $80 \%$. The Hosmer-Lemeshow test was used to determine the goodness of fit of the model, with a p-value $>0.05$ indicating that the final model was a good fit for the data (chi-square $=6.714, \mathrm{df}=8, p=.568)$. The final model as a whole explained between $38.4 \%$ (Cox and Snell R Square) and 53.2\% (Nagelkerke R Square) of the variance in the behavioural intention of parents/carers regarding NODA. Thus, roughly half of what makes parents/carers positively inclined to use the NODA system is still unknown (see Figure 7-7). As a result, the null hypothesis of this study was rejected. 
Table 7-6: Binary Logistic Regression Model (with missing data) of the factors that associate with the parents'/carers' intention to use the NODA for diagnostic assessment in Wales

\begin{tabular}{lccccc}
\hline \multicolumn{1}{c}{ Variable } & Beta & SE & Wald & OR(95\% CI) & P-value \\
\hline $\begin{array}{l}\text { Parent's /carer's } \\
\text { age }\end{array}$ & .099 & .040 & 6.190 & $1.10(1.02-1.19)$ & $.013^{*}$ \\
\hline $\begin{array}{l}\text { Performance } \\
\text { Expectancy }\end{array}$ & 1.317 & .360 & 13.385 & $3.73(1.84-7.56)$ & $.000^{*}$ \\
\hline Social Influence & 1.024 & .326 & 9.868 & $2.78(1.47-5.27)$ & $.002^{*}$ \\
\hline $\begin{array}{l}\text { Comfort with } \\
\text { using Laptop } \\
\text { computer }\end{array}$ & 1.936 & .968 & 4.001 & $6.93(1.04-46.23)$ & $.045^{*}$ \\
\hline $\begin{array}{l}\text { Comfort with } \\
\text { using Mobile } \\
\text { Phone/Smartphone }\end{array}$ & 2.589 & 1.069 & 5.868 & $13.32(1.64-108.27)$ & $.015^{*}$ \\
\hline
\end{tabular}

Note. Variables examined include: parents'/carers' demographics (age, gender, educational level), original UTAUT model and added related variables (performance expectancy, effort expectancy, social influence, facilitating conditions, self-efficacy, anxiety), diagnostic process of ASD related variables (time taken between seeking help and receiving a diagnosis of ASD, satisfaction with diagnostic process), technology experience related variables ( internet/computer skills, comfort with using desktop computer, comfort with using laptop computer, comfort with using tablet computer, comfort with using mobile phone/smartphone)

$O R=$ Odds Ratio $; \mathrm{CI}=$ confidence interval

${ }^{*} P<.05$

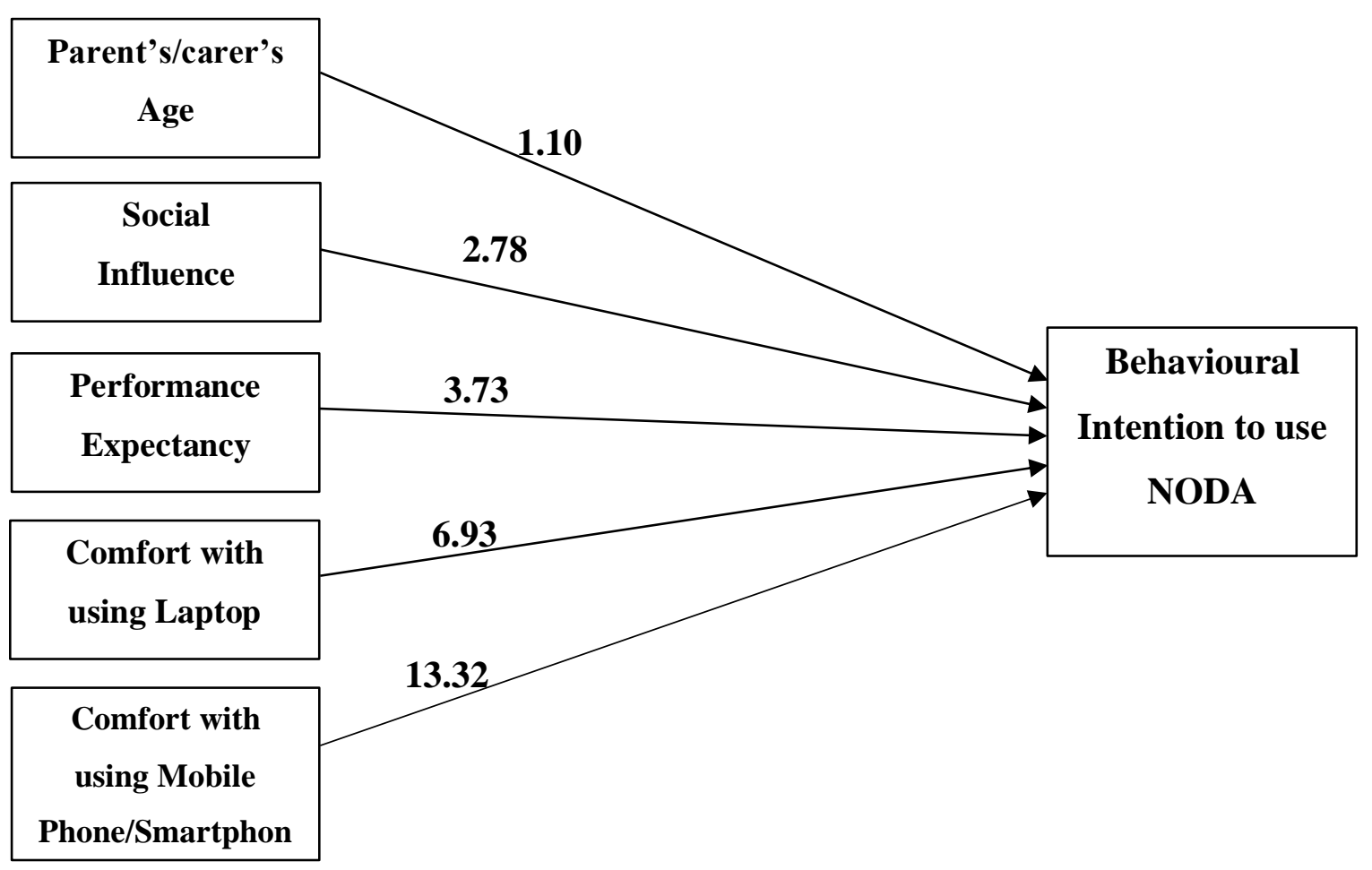

Figure 7-7: Parents/Carers Behavioural Intention to Use the NODA Model. 


\subsubsection{Parents/carers behavioural intention to use the NODA model (after imputation)}

The binary logistic regression was carried out with imputed data. As previously mentioned in Chapter 5, the missing values were assumed to be missing at random (MAR) which means that any difference between the observed values and the missing values might be described by differences in observed data (Sterne et al., 2009). This is because that the parents/carers maybe did not have enough time to complete the questionnaire. Therefore, the missing values of the dependent and independent variables that were included in the previous logistic regression model, were imputed by using multiple imputation (See Page 128). As presented in Table 7-7 the result was still the same when the binary logistic regression was carried out after imputation. Imputation did not change the findings suggesting data was missing completely at random and so missing data has the same distributions as the observed data and imputation will only increase sample size rather than needing to account for systemic differences in missingness.

Table 7-7: Binary Logistic Regression Model (with imputed data) of the factors that associate with the parents'/carers' intention to use the NODA for diagnostic assessment in Wales

\begin{tabular}{lccccc}
\hline \multicolumn{1}{c}{ Variable } & Beta & SE & Wald & OR(95\% CI) & $\begin{array}{c}\text { P- } \\
\text { value }\end{array}$ \\
\hline Parent's /carer's age & .099 & .040 & 6.190 & $1.10(1.02-1.19)$ & $.013^{*}$ \\
\hline $\begin{array}{l}\text { Performance } \\
\text { Expectancy }\end{array}$ & 1.317 & .360 & 13.385 & $3.73(1.84-7.56)$ & $.000^{*}$ \\
\hline Social Influence & 1.024 & .326 & 9.868 & $2.78(1.47-5.27)$ & $.002^{*}$ \\
\hline $\begin{array}{l}\text { Comfort with using } \\
\text { Laptop computer }\end{array}$ & 1.936 & .968 & 4.001 & $6.93(1.04-46.23)$ & $.045^{*}$ \\
\hline $\begin{array}{l}\text { Comfort with using } \\
\text { Mobile Phone }\end{array}$ & 2.589 & 1.069 & 5.868 & $13.322(1.64-108.27)$ & $.015^{*}$ \\
$\begin{array}{l}\text { Note. Variables examined include: parents'/carers' demographics (age, gender, educational level), } \\
\text { original UTAUT model and added related variables (performance expectancy, effort expectancy, social } \\
\text { influence, facilitating conditions, self-efficacy, anxiety), diagnostic process of ASD related variables } \\
\text { (time taken between seeking help and receiving a diagnosis of ASD, satisfaction with diagnostic } \\
\text { process), technology experience related variables ( internet/computer skills, comfort with using desktop } \\
\text { computer, comfort with using laptop computer, comfort with using tablet computer, comfort with using } \\
\text { mobile phone/smartphone) }\end{array}$ \\
$\begin{array}{l}\text { OR }=\text { Odds Ratio; CI = confidence interval } \\
{ }^{P} P<.05\end{array}$
\end{tabular}




\subsection{Summary}

This chapter presented the results of Study (3) that aimed to investigate the factors influencing the intention of parents/carers to use the NODA system for ASD diagnostic assessment in Wales. The chapter shared some descriptive data of their experience with technology, their perceptions on the NODA system using UTAUT/added constructs (performance expectancy, effort expectance, social influence, facilitating conditions, self-efficacy, and anxiety) and the expected usage of NODA for diagnostic assessment in Wales, if it had either been provided free by NHS or privately. The participants provided text responses to explain their reasons for acceptance of the NODA system. Univariate analysis was conducted to determine the variables associated with parent/carer behavioural intention to use NODA. A binary logistic regression analysis was also performed to examine which factors were predictive of their intention to use NODA for diagnostic assessment in Wales.

\section{Findings to be taken forward:}

- The majority of parents/carers have average computer/internet skills $(51.3 \%)$.

- The majority of parents/carers have a high level of comfort in using technological devices such as desktop computer (52.2\%), laptop computer (50.4\%) and mobile phone/smartphone (69.9\%).

- It is important to note that the overall response toward the behavioural intention to use the NODA for remote ASD diagnosis was positive (66.0\%), although $12.4 \%$ of the parents/carers felt they would not have used the NODA system.

- The findings from this study indicated that some parents/carers supported using NODA for the following reasons: speedier diagnosis, ease of use, facilitating the behavioural observations in a child's natural environment, providing a useful record to evidence behaviours, possibility of helping to receive early interventions and generally being less stressful.

- The findings from this study implied that some parents/carers did not support using NODA for the following reasons: lack of capturing complexity of issues, resistance to being filmed and behaviour masking, potential for parental recording bias, a preference by parents/carers for face 
to face interaction with professionals, privacy concerns, possibility of not suiting complex cases and uncertainty of using NODA in right way.

- It is worth noting that both parents/carers who felt they would use NODA and those who would not use it, suggested that NODA could be used in conjunction with face to face approach to improve the diagnostic process as a whole.

- The majority of respondents would have considered using the NODA system for diagnosis, if it had been provided by NHS (67\%). However, $41 \%$ would have considered using the NODA system if it had been provided privately and accepted by the NHS.

- The univariate analysis findings from this study indicate that performance expectancy, effort expectancy, social influence, facilitating conditions, and self-efficacy are associated with the behavioural intention to use NODA for diagnostic assessment.

- The logistic regression analysis model findings revealed that the age of the parent/carer, performance expectancy, social influence, comfort with using a laptop computer and comfort with using a mobile phone/smartphone play a major role in whether the parent/carer accepts the use of the NODA system for remote ASD diagnosis and assessment. This means that parents/carers would be more motivated to accept the NODA system if they felt more comfortable with using the laptop computer and mobile phone/smartphone, had higher expectations of the system's ability to reduce the time taken to obtain a diagnosis and believed that people who are important to them e.g. family members, friends, professionals) would recommend using the NODA system.

- The strongest predictor of reporting a behavioural intention to use NODA was comfort with using a mobile phone/smartphone.

- Although the Hosmer and Lemeshow goodness-of-fit test suggested that the model was a good fit for the data (chi-square: 6.714, $p=.568$ ), the model as whole explained between $38.4 \%$ and $53.2 \%$ of the variance in parents/ carers behavioural intention with regard to NODA. Thus, roughly half of what makes parents/carers positively inclined towards the NODA system is still unknown. 
Findings from the previous Chapter (6) indicate that parents/carers in Wales are dissatisfied with the ASD diagnostic process as a whole. Chapter (3) suggests that NODA has the potential to improve the diagnostic process. Taken together, these indicate that adoption of the NODA system as part of the diagnostic process would be highly beneficial for all concerned. However, user acceptance is essential for any successful implementation of telehealth (Davis, 1993; Hu et al., 1999). Therefore, findings from this study suggest that the overall response as to whether NODA would be accepted, is positive. Some parents/carers believed that NODA would be useful for providing remote ASD diagnosis. However, others considered that it may not be appropriate for all children. Hence, NODA has the potential to be used for ASD diagnosis and assessment but this varies on a case by case basis. Nonetheless, NODA might be used in conjunction with a face to face approach as screening tool for ASD to improve the diagnostic process as whole, even in complicated cases. The following chapter presents a discussion and summary of the main study findings. 


\section{Chapter 8: Discussion}

\subsection{Introduction}

The purpose of this chapter is to discuss and interpret the key findings of this thesis. The chapter will focus on explaining and investigating our findings showing how it relates to other published literature in the field. It will also highlight any new understanding or insights that have become apparent as a result of this thesis. It begins with an overview and brief findings of the scoping review (Study 1) that were previously discussed in Chapter 3. This is followed by a discussion of the Study (2) findings related to exploring parental experience with the diagnostic process in Wales. Then, Study (3) findings are discussed, which aimed to examine the behavioural intention of parents/carers to use the NODA system for ASD diagnostic assessment. Finally, it presents a summary of this chapter.

\subsection{Overview}

Previous research indicates that parents of children with ASD face a major challenge with the long delay between seeking professional help and receiving a formal diagnosis of Autism Spectrum Disorder (ASD) (Crane et al., 2015; Howlin \& Asgharian, 1999; Howlin \& Moore, 1997). Furthermore, the overall process involved in obtaining a diagnosis of ASD has often been identified as unsatisfactory for many parents (Crane et al., 2015a; Goin-Kochel et al., 2006; Howlin \& Moore, 1997; Potter, 2017; Siklos \& Kerns, 2007). Telehealth has the potential to improve access to ASD diagnostic services. In particular, telehealth offers a chance to accelerate the diagnostic process (Nazneen, Matthews et al., 2015; Reese, Braun et al., 2015; Reese, Jamison et al., 2015; Smith et al., 2017). However, new technology could not be successful if the proposed user (such as parent/carer) may not use it or is otherwise dissatisfied with it. Therefore, user acceptance has been considered to be the essential factor of the successful and sustained implementation of information system schemes, including telehealth (Davis, 1993; Dillon \& Morris, 1996; Hu et al., 1999). Determining what might affect the attitude of parents/carers toward remote diagnostic systems may help to predict future adoption of such systems and also suggest solutions that could address any potential barriers to telehealth being used for ASD diagnostic assessment. The 
purpose of this thesis was to examine if telehealth technology can be used (e.g. acceptance by parents/carers) to increase access to ASD diagnostic services and reduce the amount of time it takes for parents/carers of children with ASD in Wales, to obtain a confirmed diagnosis. As mentioned earlier, it was necessary to conduct three studies to fulfil this aim.

Firstly, a scoping review (Study 1) was conducted to examine which telehealth approaches are used in the diagnosis of ASD, whether they are feasible and acceptable, and how they compare with face to face methods. This review of the literature found there to be two methods of using telehealth: (a) a Real-Time method e.g. videoconferencing that enables teams in different areas to consult with the families and to assess the child in real time and (b) a Store-and-Forward method as Naturalistic Observation Diagnostic Assessment (NODA) system, to upload videos of the child's behaviours to a web portal that enables the clinicians to make an assessment remotely. Findings from this review of studies also found that existing telehealth systems already used in the diagnosis of autism, have been received positively by families, adults and clinicians alike and are regarded as an acceptable alternative method of diagnosis. Both remote and face to face methods produced similar diagnoses. The scoping review also suggests that the use of telehealth to diagnose ASD has the potential to greatly improve the efficiency of the diagnosis process as a whole, especially for those families living in rural area for whom access to services is far more difficult, and for those children who demonstrate some of the 'classic' behavioural characteristics of ASD. However, findings from this nascent body of literature indicate that to date, no study has examined the factors that drive the acceptance of telehealth by parents/carers of children with ASD, based on a theoretical foundation. Therefore, this study aims to fill this gap and examine the factors relating to the acceptance of telehealth, particularly the NODA system (as explained in Chapter 3) amongst parents/carers of children with ASD in Wales, using the Unified Theory of Acceptance and Use of Technology (UTAUT) model.

However, due to the supposed improvements to assessment and diagnosis services for children that were suggested as part of the Refreshed Autistic Spectrum Disorder Strategic Action Plan 2016 (Welsh Government, 2016), it was considered imperative to conduct an exploration into parental experiences of obtaining an ASD diagnosis in 
Wales, before examining the willingness of parents/carers to use the NODA system. The aim was to investigate their experience with the diagnostic process and whether they were any more satisfied with their children being diagnosed more rapidly and at earlier ages. Thus, following the scoping review, an online survey of 162 parents/carers of children with ASD in Wales was conducted comprising of two studies. The first study was conducted to explore the experience of parents/carers with the diagnostic process of ASD. The objective of the second study was to examine the factors that influence their intention to use the NODA system for ASD diagnostic assessment. The interpretations of these findings for each study, in comparison with other relevant research, is discussed in the following sections.

\subsection{Study (2): Exploring the Parent/Carer Experience in Wales of Receiving a Diagnosis of Autism Spectrum Disorder for their Children}

As we mentioned previously, the objectives of this study were to (1) explore the parent/carer experience of obtaining a confirmed diagnosis of ASD for their child in Wales, (2) examine the significant factors associated with parent/carer satisfaction with the overall diagnostic process and (3) examine predictors of parent/carer satisfaction with the overall diagnostic process. The next section considers the experience of parents/carers with the diagnostic process of ASD.

\subsubsection{Experience of parents/carers with the diagnostic process}

It was evident that most of parents/carers began to have concerns about their child's development at a very early age, with around $67.3 \%$ first noticing warning signs when their children were between 0-24 months. This percentage is higher than that reported by Crane et al. (2015) and Howlin and Moore (1997) who stated that about half of parents/carers recognised problems between 0-24 months. The key areas for parent/carer concern centred around behavioural difficulties, difficulties in playing or interacting with others, or playing alone "in his/her own world", and/or insistence on sameness. This also chimes with the earlier study (Crane et al., 2015) but differs from Howlin and Moore's (1997) research, where delay in talking or other language problems was the most commonly reported area of concerns. Almost $39 \%$ of 
parents/carers first sought help when their child was between 13-24 months. This percentage is also more than that was cited by Crane et al. (2015) where $25 \%$ of participants first began to seek help for their child between 1 and 2 years. It is possible that parents/carers in this study identified the problems and sought help at earlier ages due to the fact that their children may have had "classic" or more severe symptoms of ASD, or it might be because of an increased level of awareness of ASD within the general population. Efforts to raise the awareness of parents and improve their knowledge regarding child development, including early indications of ASD, might pave the way to receiving earlier diagnoses and intervention services.

Consistent with the previous research (Crane et al., 2015; Howlin \& Moore, 1997), this study found that most of parents/carers first turned to their health visitor to discuss their initial concerns about the development of their child, with a few initially approaching a paediatrician. When parents/carers first sought professional help and discussed their concerns, only $2 \%$ of children in the current research were given a diagnosis, however, this initial diagnosis was not certainly correct. This is similar to findings of other studies, where less than $10 \%$ of children obtained diagnoses during this initial contact (Crane et al., 2015; Howlin \& Asgharian, 1999; Howlin \& Moore, 1997). Also in line with these studies, the present study found that a substantial number of parents/carers (58.3\%) were referred to another professional after the first contact. Of concern however, is the number of parents/carers $(22.2 \%)$ who were informed that there was "no problem" or told not to worry (e.g., "they'll grow out of it"). This is comparable to the percentage of participants who cited such experience in Howlin and Moore's 1999 and Howlin and Moore's 1997 studies. The reason for such a response from the health professional is not clear, but it may suggest a lack of proper training and knowledge regarding autism spectrum disorders amongst primary healthcare providers. On the other hand, it might be due to the fact that some professionals tend to dismiss or minimise parental concerns about their child's development. Although these might be possible explanations, they do not decrease the potential negative impacts of such a response. Such findings may also reduce the possibility for access to the early intervention services. Some of the following comments were from parents/carers in the current study, with regard to their experience of discussing their first concerns with professionals: 
"Professionals unwilling to listen to concerns, as she is an intelligent girl she was seen as a child with behavioural issues, which they put down to the fact that I am a single mother and she has no contact with her father."

(Mother of a 16-year- old girl, diagnosed with Autism at the age of 15 years)

"Health visitor did not recognise my concerns. Said as a teacher I knew too much and was looking into things and he could give eye contact so definitely wasn't autistic!!!"”

(Mother of a 9-year- old boy, diagnosed with Autism at the age of 5 years)

The majority of parents/carers in this study saw five or more professionals before obtaining a formal diagnosis, which is similar to that cited by previous studies (Eggleston, Thabrew, Frampton, Eggleston, \& Hennig, 2019; Goin-Kochel et al., 2006). In the current study, children were given a confirmed diagnosis on average, at 6.93 years of age. This is slightly lower than the age cited by Crane et al. (2015) where the mean age was 7.5 years at the time of obtaining a diagnosis. This finding suggests that the average age of a child at time of diagnosis has not greatly reduced. However, it is higher than that reported in other previous studies (Eggleston et al., 2019; Howlin \& Asgharian, 1999; Howlin \& Moore, 1997). A major finding from this study was that parents/carers face a long delay of 46.27 months (3.9 years) between first seeking help and obtaining a confirmed diagnosis of ASD for their child. This finding indicates there to be a slight increase in the amount of time taken to get diagnosis, from the time of Crane et al.'s (2015) survey, where the delay was reported to be 3.5 years. The delay in this study is also longer than that found in previous studies (Chamak et al., 2011; Eggleston et al., 2019; Howlin \& Moore, 1997; Siklos \& Kerns, 2007). A possible explanation for this might be that some cases had mild symptoms that were not obvious, which meant that professionals might have needed more time to conduct the necessary assessments, in order to provide the correct diagnosis. In line with the findings of Crane et al. (2015) and Howlin and Asgharian (1999), children in this study who had been given a diagnosis of 'Asperger syndrome' faced a longer delay $(M=$ 56.67, $\mathrm{SD}=36.511)$ than those provided with the 'Autism' diagnosis $(M=46.43$, $\mathrm{SD}=43.847)$ or 'ASD' diagnosis $(M=40.02, \mathrm{SD}=31.770)$. Furthermore, these children received a final diagnosis at a later age (Asperger syndrome $M=9.28, \mathrm{SD}=2.886$; Autism $M=6.45, \mathrm{SD}=4.397$; ASD $\mathrm{M}=6.32, \mathrm{SD}=3.570$ ). Although the 'Asperger syndrome' diagnostic label has currently been excluded from the DSM-5 (American 
Psychiatric Association, 2013a), it is crucial to examine the long and challenging delay faced by those individuals who had been given the diagnostic label 'Asperger syndrome'. Another possible explanation for the longer delay in obtaining a formal diagnosis might be related to the shortage of professionals working within the field of ASD in Wales. Nevertheless, this remains a substantial period of time to wait for a confirmed diagnosis, which can have significant consequences on any early intervention services. The next section discusses the results of satisfaction levels of parents/carers with the diagnostic process, including associated factors.

\subsubsection{Factors associated with the satisfaction levels of parents/carers with the diagnostic process of ASD}

When parents/carers were asked about their satisfaction with the overall diagnostic process, $59.4 \%$ reported that they were not satisfied. This result is comparable to that described by Crane et al. (2015) where 52\% of parents were dissatisfied with their experiences. However, it differs from some published studies. For example, Eggleston et al. (2019) found that $30.8 \%$ of parents were dissatisfied. Howlin and Moore (1997) found that $49 \%$ of parents were not satisfied with the diagnostic process. Goin-Kochel et al. (2006) also described that $40 \%$ of participants were not satisfied. This suggests that most parents/carers had negative experiences with the overall diagnostic process of ASD in Wales and that the dissatisfaction rate is even higher than those found by some of the previous studies. Furthermore, when parents/carers were asked about the help/support they had been offered following diagnosis, $63.6 \%$ of them were dissatisfied and only $18.2 \%$ said that they were satisfied. Although, the percentage of those dissatisfied match that found by Crane et al.'s (2015) study, it was considerably higher than the 35\% identified by Howlin and Moor (1997). It may be that these parents/carers had high expectations regarding the post-diagnostic help, particularly those who had previously had a positive experience with the diagnostic process itself. It is crucial to notice that $44.4 \%$ of participants received no help/support during the process of seeking a diagnosis as well as the fact that $46.6 \%$ of parents/carers also received no help/support in follow-up appointments after diagnosis. However, around only $16 \%$ of parents/carers were offered direct help by professionals during the process of them seeking a diagnosis and $13.5 \%$ were provided with direct help after diagnosis. Parents/carers who had direct support during and post diagnosis, possibly 
unsurprisingly, were more satisfied with the services they had received. The results of our study show that no significant association was found between satisfaction with the post-diagnostic support and satisfaction with the overall diagnostic process. This reflects a difference from previous research, which has reported this association as being significant (Crane et al., 2015). It is likely that this finding was not replicated because of the limitations concerning around the sample used in this study, although clearly greater efforts are needed to improve the level of parent/carer satisfaction with the help/support provided following diagnosis. Providing direct and specific help to access pertinent support services e.g. arranging an appointment with an occupational therapist, instead of just signposting parents/carers to general services, is likely to result in more positive perceptions of the services offered.

Exploring the factors that were associated with satisfaction with the overall diagnostic process, the child's age when parents/carers first noticed concerns about development was found to play an important role, being related to the level of parent/carer satisfaction. Those parents/carers who first noticed atypicalities with their child development at early ages, they were more satisfied with the overall diagnostic process. This variable has not been explored by previous research. The classic symptoms of ASD may have helped some parents/carers to realise that their child had not attained the anticipated developmental milestones at early ages. These signs may have sparked their concerns and encouraged them to seek professional help early, which might have led to early diagnosis and earlier access to intervention services (McConkey, Truesdale-Kennedy, \& Cassidy, 2009; Ooi, Ong, Jacob, \& Khan, 2016). Correspondingly, this could have increased the satisfaction of those parents/carers with the overall diagnostic process. These findings highlight the importance of promoting awareness among parents/carers around the subject of child's development and the early signs of ASD, for the purpose of maximizing the chances of early diagnosis and intervention services. Another important finding was that the child's age when parents/carers first sought professional help was associated with their satisfaction with the overall diagnostic process. This finding suggests that parents/carers who sought professional help for their children when they were younger, tended to be more satisfied than those who sought help for their children at a later age. This variable has also not been tested by earlier studies. One possible explanation for this result might be that seeking help at a later age inevitably means 
receiving a confirmed diagnosis when the child is older. This may, in turn, reduce access to early intervention services, resulting in them viewing the experience more negatively. This would indicate that greater efforts are needed in order to encourage parents/carers to raise any concerns early on with the professionals, in order to increase the likelihood of the child obtaining both a diagnosis and access to early intervention services sooner. However, it is crucial to acknowledge that, although some children begin displaying autism symptoms as early as a few months old, others may start showing the signs later on in their life (Centers for Disease Control and Prevention, 2019). Consequently, parents/carers may not be in a position to seek help when their child is young, thus leading to a delayed diagnosis.

This study has been unable to demonstrate any significant association between satisfaction with the diagnostic process and a child's diagnosis, as was found in the previous research (Howlin \& Moore, 1997). Regarding the child's characteristics, current age of the child was also not associated with satisfaction with the overall diagnostic process. This result is consistent with the previous study (Howlin \& Moore, 1997). Moreover, no statistically significant association was found between satisfaction with the diagnostic process and the child's gender. One possible explanation for these results may be the lack of adequate sample size within this study. Furthermore, those characteristics of the child are maybe less relevant, when considering the wider experience of parents/carers with the overall diagnostic process of ASD.

In the current study, the majority of parents/carers (70.3\%) reported that they had experienced problems because of the numerous referrals between different professionals. The number of professionals consulted during the diagnostic process and whilst obtaining referrals, was associated with parental satisfaction with the overall diagnostic process. These findings suggest that those parents/carers who visited a greater number of professionals and experienced issues with obtaining referrals, reported higher levels of dissatisfaction. This also chimes with earlier studies (Eggleston et al., 2019; Goin-Kochel et al., 2006) and might also be related to the fact that some parents/carers were required to see many professionals in order to receive the necessary assessments for their child or might even have been provided with the incorrect diagnosis. More visits to professionals means more waiting time before 
obtaining a confirmed diagnosis, which might understandably increase levels of stress and frustration for parents/carers. One of the parents/carers shared the following comment that indicate how this was a particular problem for them:

"I was passed on a variety of professionals including an educational psychologist, an occupational therapist, locum paediatricians...I raised concerns but kept seeing different professionals so there was no continuity. Each professional referred me on to someone else. Auditory processing issues were seen as hearing problems. Fine motor skills were seen as something related to joint hypermobility. Speech problems were seen in isolation as relating to hypermobility. Toe walking was also treated by a podiatrist. No one (medically) was seeing the whole picture (except me)"

(Mother of a 9-year- old boy, diagnosed with Autism at the age of 7 years)

Satisfaction with information provided at diagnosis e.g. about the nature of ASD, suggested places to obtain support and how autism could impact the child throughout their life, also appears to be associated with the satisfaction with overall diagnostic process. That is, parents/carers who were satisfied with the information given at diagnosis were more likely to be satisfied with the overall diagnostic process than parents/carers who were dissatisfied with the information. This is similar to the findings of previous research in this regard (Crane et al., 2015; Hasnat \& Graves, 2000; Osborne \& Reed, 2008). The proportion of the current sample dissatisfied with information that they were given by professionals (40.6\%) was higher than the $33 \%$ noted by Crane et al., (2015). This result is likely due to the parents/carers having higher expectation of the information that they anticipated receiving at diagnosis. Therefore, one simple suggestion for a way in which professionals might improve parental experience with the diagnostic process, is to reconsider the ways in which they provide the information (e.g. written, verbal) and the amount and type of information. Furthermore, providing training to the professionals regarding the autism related information that should be offered to the parents/carers at diagnosis, might have an important role in enhancing parent/carer satisfaction.

Research conducted into families of children with ASD has found that the time they have to wait from reporting their initial concerns to receiving a confirmed diagnosis of ASD is very stressful (Ooi et al., 2016). The current study indicates that the majority of parents/carers found the diagnostic process stressful, with $68.4 \%$ reported that it to 
be very stressful and $21.8 \%$ as quite stressful, while only $7.5 \%$ described the process as not very stressful and $2.3 \%$ as not at all stressful. This corresponds with several studies that confirm the high level of stress experienced by parents/carers during the diagnostic process (Crane et al., 2015; Eggleston et al., 2019; Siklos \& Kerns, 2007). Furthermore, the stress of the diagnostic process was found to be associated with satisfaction with the overall diagnostic process. This finding suggests that parents/carers who found the diagnostic process stressful were more likely to be dissatisfied than parents/carers who did not find the process stressful. This is in accordance with the results of a previous surveys of parents of children with ASD, in which stress was noted as being associated with the ASD diagnostic process (Crane et al., 2015; Eggleston et al., 2019; Siklos \& Kerns, 2007). The long delay between having concerns about their child's development and receiving a formal diagnosis might be one of the reasons for this stress (Mansell \& Morris, 2004), particularly for those parents of children who without obvious ASD symptoms, as they may have experienced a longer diagnostic delay when compared to parents/carers of children with greater communication issues (Siklos \& Kerns, 2007).

Moreover, every part of the diagnostic process only serves to increase stress levels for the parents/carers involved - initially discussing their concerns regarding their child's development with the professionals, being provided with a diagnosis and then given information about the diagnosis. Indeed, the entire process of receiving an ASD diagnosis is very stressful for parents/carers and may raise their exposure to negative health outcomes, including anxiety and depression (Hayes \& Watson, 2013). Therefore, this study highlights that further work is required to make the ASD diagnostic process as helpful, comfortable and supportive as possible, from the time when a parent/carer first raises concerns with professional, through to the final diagnosis and beyond. Furthermore, an early diagnosis might be a primary contributor to reducing stress levels amongst parents/carers during the diagnostic process. This may, in turn, improve their experiences of and attitudes towards the diagnostic process as a whole.

In terms of the demographic related variables of parents/carers, the findings of our study show that age, gender, current marital status, number of children and number of children with ASD do not have significant associations with the overall diagnostic 
process. These variables have not previously been investigated. Education level was also not linked to satisfaction with the overall diagnostic process. However, this result does not support the previous research (Goin-Kochel et al., 2006). Furthermore, no statistically significant association was found between satisfaction with the diagnostic process and living area. This finding concurs with Howlin and Moore's (1997) research. It seems that demographic variables do not matter when considering satisfaction with the overall diagnostic process of ASD, especially amongst the parents/carers of children with ASD.

The current study also indicated that parents/carers had difficulties in accessing services because of a lack of eligibility (58.8\%), lack of services (45.8\%), delays in getting appointments and long waiting lists $(87.0 \%)$, difficulty obtaining information $(61.8 \%)$ and cost related problems (28.2\%). These percentages were higher than those reported in earlier research conducted by Daniels et al., 2017, who found that carers of children with ASD had difficulty in accessing services due to problems getting appointments (29\%), lack of eligibility (31\%), lack of services (35\%) and a lack of information $(31 \%)$. However, the percentage of parents/carers who reported that they had difficulty because of the costs involved was similar to that found in this study (31\%). This previous research (Daniels et al., 2017) found that some carers identified other difficulties that were related with having limited access to school as well as issues with teaching staff or school administration. However, the current study found that among those participants who provided free text responses to "other difficulties or delays for any other reason" difficulties were generally experienced because of the following reasons: waiting time, multiple referrals to different professionals, cancelled appointments, lack of services and shortage of professionals, inadequacies with Child and Adolescent Mental Health Services (CAMHS) provision, difficulties in finding the relevant information and issues related to professionals (e.g. paediatrician, GP, health visitor, speech and language therapist and educational psychologist) such as their reluctance to listen to parental concerns, non-recognition of parental concerns, delays in providing the statement of special educational needs (SEN), forgetting to make referrals, sending referrals to another professional with some errors, and losing paperwork or assessments required to make the diagnosis. Although the difficulties relating to lack of services, waiting time and issues in finding the necessary information were mentioned within the multiple-choice questions within the study's 
questionnaire, the fact that parents/carers highlighted them for a second time in their text responses, may suggest how hard it was for them to obtain the services they needed for their children. Overall, these findings suggest that parents/carers of children with ASD in Wales, face considerable barriers when attempting to access services.

The results also revealed that difficulties of accessing services due to problems in obtaining information was linked to satisfaction with the overall diagnostic process. Participants who had difficulties for this reason were, perhaps unsurprisingly, more likely to be dissatisfied than those who had not similar difficulties. This variable has not previously been investigated as being indicator of satisfaction levels with the diagnostic process. One of the parents/carers shared the following comment, which shows how difficult it was for them to obtain the information they needed:

"Finding the information you need is ridiculously hard. There is no one stop shop $\&$ no one point of contact to walk you through it all, which when your resources are already depleted is exhausting. Accessing services such as occupational therapist or respite is a lottery and extra work, when I feel it should be automatically offered following a diagnosis. You should not have to fight for everything you need and prove your worthiness to have it"

(Mother of an 8-year- old boy, diagnosed with ASD at the age of 5 years)

Therefore, it is clearly necessary to make the process of finding the right information easier for parents/carers in Wales, which may in turn improve their satisfaction with the overall diagnostic process.

Furthermore, significant association was found between the difficulties accessing services due to lack of services themselves, and satisfaction with the overall diagnostic process. In other words, parents/carers who reported that they had difficulties accessing services because there were so few of them, were more likely to be dissatisfied with the diagnostic process as a whole than those who reported that they had no such difficulties. This variable has also not previously been investigated. One possible explanation for this finding could be related to the limited supply of providers working within the field of autism in Wales. This may have increased the waiting time of parents/carers attempting to access services for their children e.g. diagnostic assessment, and could have meant a late diagnosis. This may, in turn, have decreased the satisfaction levels with the overall diagnostic process amongst parents/carers, some 
of whom shared the following comments, which indicate how difficult it was for them to access the services they needed:

"Did not see paediatrician for over a year as no doctor available"

(Mother of a 11-year- old boy, diagnosed with ASD at the age of 8 years)

"No paediatrician employed in our area"

(Mother of a 14-year- old boy, diagnosed with Asperger syndrome at the age of 11 years)

"Services are overworked or non-existent"

(Mother of a 10-year- old girl, diagnosed with ASD at the age of 3 years)

A shortage of professionals may result in decreased access to the autism-related services. As a result, families who have concerns about their child's development have to travel long distances or attend privet clinics in order to obtain assessment and evaluation (Oberleitner et al., 2007; Reese, Braun et al., 2015). As well as meaning increased travel and costs, such professionals might not have a clear awareness of the resources in the area where the families live (Reese, Braun et al., 2015). Clearly, parents/carers of children with ASD might well encounter a lack of essential services for their children. Such finding only serve to further highlight the need to increase the number of professionals working in the field of autism in Wales, both during and after the diagnostic process. This might well improve attitudes and experiences of parents/carers. Furthermore, use of telehealth could help in rapidly expanding autismrelated services to reach a greater population, particularly enabling early detection and assessment of ASD services in rural places (Oberleitner et al., 2007; Reese, Braun et al., 2015).

Furthermore, the study results revealed that a child's age at time of diagnosis was associated with parent/carer levels of satisfaction with the overall diagnostic process. That is, the parents/carers of children who were diagnosed earlier appeared to be more satisfied with the diagnostic process than those who obtained a diagnosis later. The findings of this research mirror those of previous studies that have examined the association between a child's age at diagnosis and parental satisfaction with the diagnostic process on the whole (Crane et al., 2015; Howlin \& Moore, 1997). The next section discusses the predictors of parent/carer satisfaction with the diagnostic process of ASD. 


\subsubsection{Predictors of parent/carer satisfaction with the diagnostic process of ASD}

A major finding from this survey was that the time taken to obtain a confirmed diagnosis was associated with satisfaction levels. It was also the only significant predictor of satisfaction with the overall diagnostic process that appears in the binary logistic regression analysis. This finding suggests that parents/carers who had longer wait times to get a diagnosis were more likely to be dissatisfied with the process than those who waited for a shorter period of time. This finding is in line with earlier research conducted in the area of diagnostic process of ASD (Crane et al., 2015; Howlin \& Moore, 1997; Siklos \& Kerns, 2007) which has consistently identified it as one of the significant variables influencing parental satisfaction with the diagnostic process as a whole. As previously mentioned, on average, parents/carers in the current study typically experienced a delay of 46.27 months between first seeking professional help and receiving a confirmation of ASD diagnosis for their child. It has been indicated that delay in obtaining a diagnosis of ASD could be more likely when the child's disabilities are less apparent (Siklos \& Kerns, 2007). Children in the current study with classic symptoms of ASD, such as language and behaviours impairments, may have received a diagnosis earlier than those children whose symptoms were less noticeable. As mentioned previously that, it was found that children who had been diagnosed with the Asperger syndrome diagnosis faced a longer delay than those diagnosed with autism or ASD. This supports findings from previous research in this area (Howlin \& Asgharian, 1999). Furthermore, some cases might have needed to be reassessed as the professionals were possibly unable to provide a correct diagnosis early on. A shortage of professionals working in the field of autism in Wales could also be one of the reasons for long waiting times between seeking help and obtaining a diagnosis.

The extended wait to receive a final diagnosis could well cause stress for the parents/carers and other family members (Mansell \& Morris, 2004), increasing the possibility of anxiety and depression (Hayes \& Watson, 2013; Siklos \& Kerns, 2007). Delay in diagnosis may also prevent access to early intervention services, which are critical for positive outcomes (Matson, 2008; McEachin et al., 1993; Rattazzi, 2014; Siegel, 2008; Smith et al., 2017). Furthermore, the long wait times might stir negative emotions in parents/carers, particularly with regard to their own uncertainty, confusion, as well as feelings of guilt that they cannot improve their child's behaviours 
and that their child may not be able to access early intervention services (Davidovitch, Glick, Holtzman, Tirosh, \& Safir, 2000; Wiggins et al., 2006). Parents/carers in this study who had experienced a long delay and whose children were diagnosed at a later age, may had experienced these same previous feelings, which in turn, may have increased their dissatisfaction about the diagnostic process as a whole. Clearly, more needs to be done to improve the experiences of parents/carers during the diagnostic process in Wales, and decreasing the time between seeking professional help and receiving a formal diagnosis would be a substantial step in the right direction. The sooner that parents/carers can receive a diagnosis for their child, the sooner they can access to services, particularly the early intervention services. Parents/carers feelings might also feel less anxious if they were able to receive an explanation for their child's problems sooner rather than later (Howlin \& Moore, 1997; Mansell \& Morris, 2004). Nevertheless, it is crucial to mention that in some cases, further time might be necessary in order to allow professionals to conduct the reassessments after a certain time, in order to ensure a correct diagnosis.

Telehealth could be one of the possible solutions that may improve access for parents/carers to the ASD diagnostic services and reduce the time required to secure a diagnosis, as we explored in the scoping review (Chapter 3). As we mentioned earlier that, although this review suggests that telehealth was acceptable for parents, findings from that particular body of literature indicated that to date, no study had examined the factors influence whether the parents/carers of children with ASD will accept telehealth as a means of diagnostic assessment, on a theoretical basis. The next section discusses the results of Study (3) which aims to examine the degree to which parents/carers will accept use of the NODA system to support diagnostic assessment of their children. 


\subsection{Study 3: Exploring the Acceptance Levels of Parents/Carers in Wales of Using NODA for ASD Diagnostic Assessment of their Children.}

The objective of this study was to examine the predictors of parent/carer intention to use the NODA system for ASD diagnostic assessment, by applying an extended UTAUT model. More crucial than modifying or extending an existing model, the main goal of this study was rather to provide the foundation for a fuller understanding of whether telehealth could be accepted for ASD diagnostic assessment by parents/carers. Although this current study aimed to examine existing relationships, it is important to simultaneously investigate other potential factors that may influence intention to use the NODA system. It is to be hopes that upcoming studies may benefit from what has been revealed in this research. For example, future scholars may decide not to include determinants that had only a relatively minor impact on the behavioural intention. Others may choose to examine new constructs that were not originally studied, and may provide new and interesting results.

This study suggests that the majority of parents/carers (66.0\%) felt positively about using the NODA system, if it had been available before their child's diagnosis. The high percentage of parents/carers who felt that they would have used the NODA system, as was found in our study, is a key finding. This is in line with previous study that aimed to explore the attitudes of parents and diagnosticians regarding their experience of the NODA system (Nazneen, Matthews et al., 2015). Similar positive findings were also suggested by earlier studies (Breil et al., 2019; Juarez et al., 2018; Van der Vaart et al., 2016; Zagzoug, 2015). The analysis yielded five significant predictors in the final extended UTAUT model, with $38.4 \%$ (Cox and Snell R Square) and $53.2 \%$ (Nagelkerke R Square) of the variance explained by the behavioural intention to use NODA by parents/carers. Although the explained variance is not as high as the $70 \%$ variance explained in the original UTAUT model, it is considered a good start towards a better understanding of acceptance of the NODA system amongst parents/carers. The following sections will discuss the findings that related to the existing direct determinants in the UTAUT model, along with additional determinants. The reasons for the perceptions of parents/carers toward the NODA system will also be discussed. 


\subsubsection{Existing direct determinants within UTAUT model}

The next section discusses the findings of existing direct determinants within the UTAUT model, including performance expectancy, social influence, effort expectancy and facilitating conditions.

\subsubsection{Performance Expectancy}

The majority of parents/carers felt that the NODA system would have been a useful way to obtain an ASD diagnosis, if it had been available prior to their child's diagnosis. This is similar to the findings of previous studies which aimed to examine parental willingness to use some of healthcare technologies (De Graaf et al., 2014; N. Nazneen et al., 2015b; Sobowale, Nguyen, Weiss, Van, \& Trung, 2016). Nazneen, Matthews et al. (2015) indicated that some parents found the NODA system to be a useful tool for conducting remote ASD diagnosis. Sobowale et al. (2016) also revealed that many parents suggested that internet intervention would be useful in order to obtain evidence-based psychotherapy treatment for their youth.

Performance expectancy was found to be a factor associated with parent/carer intention in the univariate analysis, and influenced how they felt about using the NODA system. This suggests that they would have considered using the NODA system if they had had a clear understanding of the associated benefits, such as a shorter waiting time to receive a diagnosis and increased chances of accessing early intervention services. This chimes with the Venkatesh et al. (2003) study and with previous findings of the effect of performance expectancy on the intention of such parents to adopt an online programme for Applied Behavioural Analysis (ABA) intervention methods (Zagzoug, 2015). This finding is also consistent with a number of previous studies conducted among different population, which aimed to also examine the intention to use certain healthcare technologies based on the UTAUT model (Breil et al., 2019; Cimperman et al., 2016; Hoque \& Sorwar, 2017; Lin et al., 2016; Quaosar et al., 2018; Rho et al., 2015). For example, Cimperman et al. (2016) revealed that performance expectancy has a direct impact on whether older users adopt home telehealth services. This is consistent with further evidence, which found that performance expectancy had a positive effect on whether patients accept the use of 
telehealth for management of diabetes mellitus (Rho et al., 2015) and electrocardiogram (ECG) monitoring (Lin et al., 2016).

As these findings confirm that performance expectancy has a significant influence on behavioural intention to use the NODA system, it is important to clarify the benefits of using NODA for parents/carers and to highlight any of its' characteristics which may support the users. This is likely to encourage prospective users to accept the NODA system and motivate them to use it to obtain a diagnostic assessment for their child. Referring back to the second chapter, which outlines the problems and challenges faced by parents/carers when seeking to obtain a diagnosis for their child, it is obvious why any benefits should be clearly communicated. If the advantages are not clearly outlined to the parents/carers, they may be reluctant to adopt such technologies.

\subsubsection{Effort Expectancy}

The majority of parents/carers in the current study felt that the NODA system would be easy to use. Similar findings came from earlier literature (Nazneen, Matthews et al., 2015; Nazneen, Rozga et al., 2015) pointed out that parents without prior experience were able to use NODA Smart Capture easily, during the in-field evaluation. A study by de Graaf et al. (2014) suggested that most of the parents found the Hemangioma Treatment Plan (HTP), a type of eHealth intervention which was developed to treat Infantile hemangiomas, was an easy to use. There are some inconsistences regarding the impact of effort expectancy. Some studies found there to be influence on behavioural intention based on the UTAUT model (Breil et al., 2019; Cimperman et al., 2016; Dai et al., 2020; Hollier et al., 2018; Hoque \& Sorwar, 2017; Quaosar et al., 2018; Rho et al., 2015; Van Houwelingen et al., 2018) while others none (Hu et al., 1999; Lin et al., 2016; Macdonald et al., 2019; Zagzoug, 2015). The findings of the current study were in line with latter group of studies, as the influence of effort expectancy on the intention of parents/carers to use NODA was insignificant, although it was associated with the intention in univariate analysis. It is interesting to note that this study produced results which corroborate the findings of previous research, which was conducted with the aim of examining the intention of using a telehealth training system among such population (Zagzoug, 2015). Macdonald et al. (2019) also showed 
that effort expectancy did not affect patients resolve to adopt smart insole technology, designed for self-management of diabetes. The finding from the current study suggests that difficulty or ease of use of the NODA system appears not to play an influential role on the population sample. The parents/carers might have been willing to use the NODA system if they felt that it would be helpful in obtaining an ASD diagnosis, regardless of how difficult it was. In the current study, the majority of parents/carers reported that they had average computer/internet skills $(51.3 \%)$ and $29.2 \%$ reported that they had advanced computer/internet skills. This could explain why effort expectancy was not a predictor of behavioural intention to use the NODA system for parents/carers.

\subsubsection{Social Influence}

The findings of the current study also show that social influence is a factor associated with behavioural intention in the univariate analysis, and a predictor of the intention to use NODA for diagnostic assessment. This suggests that family members, friends and professionals (e.g. GP, educational psychologist, clinical psychologist, psychiatrist, speech and language therapist, teacher) appear to have an influential role for the parents/carers. This is in line with the study by Venkatesh et al. (2003) amongst others, which also highlighted the significant effect of social influence (Dai et al., 2020; Hennemann et al., 2016; Hoque \& Sorwar, 2017; Kim et al., 2018; Lin et al., 2016; Quaosar et al., 2018; Zagzoug, 2015). A study by Dai et al. (2020) for example, revealed that effort expectancy was one of the predictors that was statistically significant in explaining the perceived behavioural intention to use healthcare wearable devices amongst patients with dementia, as reported by caregivers. However, this stands in contrast to previous evidence, where this predictor was not believed to be significant (Adenuga, Iahad, \& Miskon, 2017; Cimperman et al., 2016; Hoogenbosch et al., 2018). A study by Adenuga et al. (2017) demonstrated that social influence had no significant effect on the intention to use telehealth technology. However, this study was comprised of clinicians, who may have had a high level of autonomy. The nature of the population in the current study might be a reason for this contrary result. A positive attitude of important others towards the efficacy of telehealth and the willingness of professionals to recommend such technologies, can encourage parents/carers to accept and use such technology. Professionals, in particular, could play a significant role as the gatekeepers of adoption of telehealth for 
ASD diagnosis. Given that many parents/carers who will be recommended of NODA by providers, they could, in turn, be more willing to use NODA to obtain diagnostic assessment for their child. Facilitation of acceptance must therefore, involve pertinent stakeholders as important mediators for adoption of the NODA system.

\subsubsection{Facilitating Conditions}

The results showed that facilitating conditions were associated with behavioural intention in the univariate analysis, but it was not a predictor of their intention to use the NODA system. This outcome suggests that assistance, knowledge and resources that are necessary to use the system (e.g. Internet, computer, smartphone/mobile phone) did not significantly influence the intention of parents/carers to use the NODA system for diagnostic assessment. This finding is consistent with some acceptance studies that apply the UTAUT model, which show non-significant effect (Hennemann et al., 2016; Hoque \& Sorwar, 2017; Quaosar et al., 2018). Nevertheless, the influence of facilitating conditions on intention to use or actual use of technology has been shown to be significant in several previous studies (Cimperman et al., 2016; Dai et al., 2020; Kim et al., 2018; Lin et al., 2016; Rho et al., 2015; Van der Vaart et al., 2016; Zagzoug, 2015).

However, it should be noted that research by Venkatesh et al. (2003) found that a facilitating condition was significant in predicting actual usage rather than behavioural intention, and this becomes insignificant in predicting behavioural intention when effort expectancy and effort expectancy determinants are present in the same model. It has also been indicated that the effect of facilitating conditions decreased with more experience and/or knowledge of computers and Internet (Venkatesh et al., 2003). As mentioned previously, most of the parents/carers in this study reported that they have either average or advance computer/internet skills. In addition, it would seem that resources were widely available and accessible to the majority of the parents/carers, as $92 \%$ of them felt that they did have access to resources which would allow them to use the NODA system. This may explain why facilitating conditions were not a predictor of behavioural intention to use the NODA system for ASD diagnostic assessment. 


\subsubsection{Added direct determinants in amended UTAUT model}

The next sections discuss the results of the added direct constructs in the amended UTAUT model that include anxiety, self-efficacy, demographic related variables (age, gender, level of education), diagnostic process of ASD related variables (time taken to get diagnosis and satisfaction with overall diagnostic process) and technology experience variables (computer/internet skills, comfort with desktop computer, laptop computer, tablet computer and mobile phone/smartphone devices).

\subsubsection{Anxiety}

The majority of parents/carers in the current study gave negative responses (disagree, strongly disagree) regarding questions around anxiety. Most of them reported that they would not feel apprehensive about using the NODA system, would not feel scared about losing a lot of information by hitting the wrong key and would not hesitate to use it for fear of making mistakes they could not correct. The results indicated that anxiety was not associated with behavioural intention, as well as not influencing whether or not NODA system might be used among parents/carers. This suggests that anxiety level would not either reduce or increase the intention of parents/carers to use the NODA system. It is to be expected that outcome anticipations of using NODA surpassed the anxiety of using such an information technology system. This finding corresponds with the result obtained both when the UTAUT model was developed (Venkatesh et al., 2003) and with previous studies (John, 2013; Macdonald et al., 2019; Ryu, Kim, \& Lee, 2009). However, this is in contrast to some previous research which found that anxiety had a negative influence on the acceptance of using technology (Chang \& Im, 2014; Guo et al., 2013; Hoque \& Sorwar, 2017; Or \& Karsh, 2009). Earlier studies on computer anxiety point out that limited technological experience and computer skills may increase the anxiety of users (Chua, Chen, \& Wong, 1999; Glass \& Knight, 1988; John, 2013; Wagner, Hassanein, \& Head, 2010). In other words, without adequate experience with computers and internet, users are concerned that interacting with technology might lead to errors that could not be corrected. For example, Hoque and Sorware (2017) revealed that anxiety has a significant influence on the adoption of mHealth. The authors indicated that respondents have limited technological experience and showed that using technologies such as mobile phones for healthcare related purposes is still a relatively new conception in Bangladesh, 
which may have increased anxiety levels, which is in turn, likely to negatively influence their willingness to use such technology.

However, it is important to note that use of digital technology for health in Wales is not a new concept. As stated earlier, the National Survey for Wales reported that $87 \%$ of the population had access to the internet at home (Welsh Government, 2019). Furthermore, it has emphasised that two thirds of the Welsh population are using technology to support their health (Davies et al., 2019). The most frequently stated activities included finding information about general health, health services and using technology to self-diagnose a health condition. In addition, one third of the population are using technology to monitor their health. The most common reported actions were pertaining to physical health, heart rate and stress or anxiety levels (Davies et al., 2019). In particular, with reference to this study, most of the participants reported that they had either average or advanced computer/internet skills. This could be a possible explanation for these results.

\subsubsection{Self-Efficacy}

The majority of the participants gave positive responses (strongly agree, agree) towards the self-efficacy. They had positive belief in their own ability to collect and share video evidence of their child's behaviour, to collect and share video evidence if they could call someone for help if they got stuck (e.g. Help line) and to collect and share video evidence if they were provided with the built-in facility (e.g. manual, sample videos and instructions about the system) to help them. However, higher computer self-efficacy among parents/carers may not necessarily indicate an increased intention to use the NODA system. The results revealed that although self-efficacy was associated with intention in the univariate analysis, it was not found to be a predictor in explaining the acceptance of using NODA among parents/carers. This suggests that the belief of parents/carers in their own ability to collect and share video evidence of their child's behaviour from home, would not have an impact on their intention to use the NODA system. This finding is comparable to the results obtained when the UTAUT model was developed (Venkatesh et al., 2003) as well as those of other prior studies applying the UTAUT model in order to examine whether patients intended using some types of healthcare information technologies, such as mHealth apps for self-management of hypertension (Breil et al., 2019) and a web-based 
aftercare system (Hennemann et al., 2016). However, this runs contrary to the findings of some previous research, which found that self-efficacy plays a role in the behavioural intention to use videoconferencing as part of telehealth (Van Houwelingen et al., 2018) and in the use of smart house technology with an iPad (Alvseike \& Brønnick, 2012). Tellingly, the people taking part in these studies were elderly (65 years old and over), who may have had less control over such information communication technologies. It seems that these findings may have been influenced by the nature of population. Moreover, previous studies revealed that basic computer knowledge and experience is related to computer self-efficacy (John, 2013), suggesting that the individual or user with more computer knowledge is more likely to be confident in his ability to complete the task successfully. The nature of the population in the current study is different, as the average age of parents/carers was 38 years old. Moreover, the majority of respondents reported that they were comfortable with using various types of technological devices such as the desktop computer, laptop computer, tablet computer as well as mobile phone/smartphone. This may explain the fact that self-efficacy has no significance on the parents/carers intention to use the NODA system.

\subsubsection{Demographic related variables}

This section discusses the impact of demographic variables (age, gender, educational level) on the intention of parents/carers to use the NODA system for diagnostic assessment.

\section{Gender}

Although some studies have found that gender can influence whether or not a patient chooses to use certain types of healthcare communication technologies (Hennemann et al., 2016; Kontos et al., 2014), the current study revealed that gender does not play a significant role in the prediction of parent/carer intention to use the NODA system. This finding is consistent with many previous studies (Albrecht et al., 2017; Breil et al., 2019; Cimperman et al., 2016; Dou et al., 2017; Eichenberg et al., 2013; Kim et al., 2018; Zagzoug, 2015). For example, the study by Zagzoug (2015) found that gender did not influence whether the parents of children with ASD decide to use the 
online intervention program. Albrecht et al. (2017) revealed that gender was not a significant predictor of intention to use smart devices in health-related context among patients as well as general practitioners. Similar findings were noted in the study conducted by Eichenberg et al., (2013) which examined the readiness of participants to use online counselling and media-assisted psychotherapy. It is should be noted that most of the participants in the current study were females. Demographic characteristics of the sample indicated that $95 \%$ of respondents were female and only $4.9 \%$ were male.

\section{Age}

Contrary to the findings of prior research (Cimperman et al., 2016; Dou et al., 2017; Kim et al., 2018; Zagzoug, 2015), the current study revealed that age did have an influence on the intention of parents/carers to use the NODA system for ASD diagnostic assessment. This is in line with previous studies that were carried out on specific healthcare technologies in similar contexts (Albrecht et al., 2017; Breil et al., 2019; Czaja et al., 2006; Eichenberg et al., 2013; Kontos et al., 2014). However, what is surprising is that age in this study had a positive influence on behavioural intention to use telehealth and this, unlike previous findings that revealed a negative influence, suggests that older parents/carers were more likely than younger adults to accept use of the NODA system for diagnostic assessment. One possible explanation for this may be that older parents/carers have experienced more difficulties and have waited a lot longer for a confirmed diagnosis for their child, and because of this their behavioural intention was more positive toward using the NODA system. They may have been willing to try any diagnostic method that could identify their child's problem, get a swifter diagnosis and benefit from accessing early intervention services.

In our study, the actual system was not available for participants to try. Instead, videos and pictures that demonstrated how the NODA system works was presented to all participants taking part in the survey. Therefore, another possible explanation is that younger parents/carers may be more familiar with general technologies as a whole, and the description of the NODA system concept, including the videos and pictures provided in the questionnaire, may not be sufficient to persuade them to accept that such technology to be used for diagnosis of ASD. Hence, those responsible for 
designing telehealth services that aim to support the diagnostic assessment of ASD, such as the NODA system, should take into considerations the age of all possible users.

\section{Level of Education}

As earlier mentioned, $50 \%$ of participants left school at 18 or 16 years old with qualifications, $28.4 \%$ had a Bachelor degree, 9.3\% had Master degree, 2.5\% had a Doctorate degree and only $5.6 \%$ had no qualification at all. Contradicting the findings of previous studies (Albrecht et al., 2017; Eichenberg et al., 2013; Hennemann et al., 2016; Zagzoug, 2015), the results of this study indicate that level of education did not appear to be a significant predictor of the intention of parents/carers to use the NODA system. Cimperman et al. (2016) drew a comparable conclusion. In their study, education level was not found to significantly influence behavioural intention to use home telehealth services. It seems that the education level does not contribute to increase or decrease whether parents/carers will accept use of the NODA system for diagnostic assessment.

\subsubsection{Diagnostic process of ASD related variables}

This section discusses the impact of some diagnostic process of ASD related variables on the parent/carer intention to use the NODA system for diagnostic assessment, including the time taken to obtain a diagnosis and satisfaction with the overall diagnostic process. The current study revealed that satisfaction with the overall diagnostic process and the time taken to obtain a diagnosis did not influence parent/carer intention to use the NODA system for diagnostic assessment. Based on these findings, whether parents/carers decide to accept or reject the NODA system was not influenced by satisfaction with the diagnostic process and the time taken to obtain a diagnosis. This may suggest that even those parents/carers who were satisfied and who received a diagnosis for their child earlier may would have used the NODA system. 


\subsubsection{Technology experience related variables}

This section discusses the findings of the technology experience related variables including computer/internet skills, comfort with desktop computer, laptop computer, tablet computer and mobile phone/smartphone devices. Contrary to the findings of previous research (Berkowsky et al., 2018; Chang \& Im, 2014; John, 2013; Zagzoug, 2015; Zhang et al., 2012), in our study computer/internet skills did not significantly predict whether parents/carers would accept the NODA system. This suggests that the decision by parents/carers to accept or reject such a system is not influenced by computer/internet skills. However, this is not uncommon amongst studies of intention to use technology. In one systematic review of the acceptance of health information technology among patients (Or \& Karsh, 2009), computer/internet skills construct was not associated with increased acceptance in some of the reviewed studies. As mentioned earlier, the majority of parents/carers in this study rated their computer/internet skills as either average or advanced. In addition, the use of computers and accessing the internet for healthcare purposes is not a new concept in Wales (Davies et al., 2019). Therefore, computer/internet skills may not be a barrier that hinders acceptance of telehealth services to obtain a diagnosis for their child. This is contrary to findings from some research, which suggest that the users' acceptance of the web self-service application may be hampered by a lack of access to the internet and inadequate computer skills (Zhang et al., 2012). Berkowsky et al., (2018) also drew a similar conclusion.

Furthermore, the majority of the parents/carers felt that they were comfortable with using desktop computer, laptop computer, tablet computer and mobile phone/smartphone. However, this study revealed that comfort with laptops and comfort with mobile phones/smartphones were significant predictors of intention to use the NODA system. In particular, comfort with mobile phones/smartphones was the strongest predictor of parents/carers behavioural intention to adopt this system. Based on these findings, parents/carers who reported that they were comfortable with using laptops as well as mobile phones/smartphones, showed positive intention toward using NODA, when compared with parents/carers who reported that they were not comfortable with such devices. Our findings are consistent with the results of previous studies (Eichenberg et al., 2013; Koivumäki et al., 2008; Zagzoug, 2015). Zagzoug's 
study (2015) demonstrated that intention to use telehealth was significantly higher in those parents who were more familiar with using devices such as mobile phones/smartphones and laptop computers. Similarly, people who were already using the mobile phones and computer devices were more open to using online counselling and internet-assisted psychotherapy (Eichenberg et al., 2013). Providing adequate information and instructions that clearly explain the basic functions of such electronic devices as mobile phones/smartphones and laptop computers (e.g. where the on/off button is located and how it is used, capturing and sending images or videos) may help parents/carers to become more familiar with such devices, which in turn might produce favourable results regarding their behavioural intention to use the NODA system for ASD diagnostic assessment.

\subsubsection{Potential reasons for parent/carer perceptions toward the NODA system}

Some participants divulged some reasons behind their perceptions toward the NODA system. Speedier diagnosis was one of the main reasons given by those parents/carers who felt that they would have used the system, if it had been offered prior to their child's diagnosis. As the average delay between the parents/carers initially seeking help and the child receiving a diagnosis was 46.27 months, and the majority of our research sample were dissatisfied with the time taken to obtain a diagnosis (63.2\%), this could provide a possible explanation for this indication. It is worth emphasising that reducing waiting time is one of the essential benefits that is provided by telehealth (Caffery, Farjian, \& Smith, 2016). This is consistent with a previous study by Nazneen, Rozga et al. (2015) in which interviews were conducted to obtain feedback from stockholders about the concept of an asynchronous system of video collecting and sharing of in-home behavioural evidence. The parents anticipated that such a concept could better connect parents and clinicians, leading to a quicker assessment of the child. There were similar findings in evaluation of the NODA system, as some of the parents who had previously gone through the in-person diagnosis process for their older children, thought that the NODA system might enable families to receive a diagnosis sooner (Nazneen, Matthews et al., 2015). Furthermore, some participants in this study anticipated that the NODA system would be easy to use. This is in line with the previous work looking at the attitudes of adults towards the telehealth system for remote autism assessment (Parmanto et al., 2013). Nazneen, Matthews et al. (2015) 
and Nazneen, Rozga et al. (2015) also revealed that most of the parents found that the NODA system design allowed them to easily capture evidence of child behaviour and send it to the diagnosticians for them to complete the diagnostic assessment, even without any prior training. As parents/carers may potentially use this system on an everyday basis, a simple design is imperative, to ensure that this does not add burden to their already significant responsibility of care.

Consistent with the previous reserach (Nazneen, Matthews et al., 2015), parents/carers in this study who felt they would have used the system also thought that video recorded at home would allow clinicians to observe the child's behaviour in a natural environment which might not be possible in a clinic. It would also allow parents/carers to provide evidence of their child's behaviour. In addition to this, it is important to further highlight that with the NODA system, diagnostic assessment does not rely only on recorded behaviours from one or two undirected videos and any systems that try to do so might be less reliable. With NODA system, parents are directed as to how to record particular scenarios that occur naturally in their homes and are provided with instructions about how to make opportunities for their child to express their usual social interaction (Smith et al., 2017). Moreover, some participants in the current study who had a positive perception toward using NODA, thought that diagnostic assessment by using such a system might also be less stressful for children and families. As we mentioned in Study (2), the majority of parents/carers reported that they found the ASD diagnostic process stressful. Adoption of the NODA system might provide an answer. It has been highlighted that the process of obtaining a diagnosis by traditional face to face methods could be stressful for some parents as well as those individuals who might later be diagnosed with ASD (Crane et al., 2015a; Hayes \& Watson, 2013). The need to travel long distances to obtain a diagnostic assessment for their child because of a shortage of clinicians, can also lead to further stress (Oberleitner et al., 2007; Reese, Braun et al., 2015) as might long waiting times (Crane et al., 2015). Using such an approach may help to decrees the waiting time and this may, in turn, decrease stress levels amongst families. Another potential reason to use a remote diagnostic system that was highlighted by some parents/carers, was the fact that it may increase the opportunity for the child to receive early intervention serveries. Earlier studies have stressed the point that early intervention may reduce developmental delays and improve future learning capabilities (Dugger, 2012). 
However, concerns about privacy was one of the reasons behind negative parental perceptions toward the NODA system. This corroborates previous research, which suggests that 8 out of 44 parents had concerns around video sharing, but they did then agree to record their child's behaviour if policies regarding sharing made clear who would have a right to access such data (Nazneen, Matthews et al., 2015). Therefore, robust policies concerning video sharing are imperative to persuade parents/carers to use a remote ASD diagnostic system. Some participants were also concerned that some children might be able to change their behaviours if they realised they were being observed. While, Nazneen, Matthews et al. (2015) indicated that most of the parents fed back that children got used to the NODA SmartCapture after a short period of time and subsequently started to ignore it. These parents suggested that using the back camera rather than the front camera and mounting the device (e.g. on a smartphone) rather than carrying it, allowed them to manage any reactions to the device. However, as some diagnosticians have pointed out, it is crucial to emphasise that although reducing the child's reactivity is essential in order to observe natural behaviour, it does not certainly mean that a recorded video is invalid. Furthermore, chances of reactivity can even occur during face to face diagnostic assessments (Nazneen, Matthews et al., 2015; Smith et al., 2017).

Parent/carer concerns about recording improper videos for diagnostic assessment was another reason given, which might be because of unfamiliarity with working with remote diagnostic communication systems. However, as we stated previously, the NODA system has clear instructions to direct parents as to how they should interact with their child. It also enables parents/carers and clinicians to asynchronously communicate, and so if parents/carers upload a video that cannot be used for diagnostic assessment, the clinicians can guide them to record another video evidence that could be clinically meaningful (Nazneen et al., 2016). Moreover, some parents/carers highly valued face to face contact with the clinicians, which they thought was vital for effective discussion and feedback. The NODA system was not designed to eliminate the need for further appointments and assessments (Smith et al., 2017), and so they would be able to discuss any concerns they might have related to the diagnosis of their child. In addition, some enquiries could be asked asynchronously via the NODA system. Some other parents/carers anticipated that the NODA system could not capture 
all of the issues and may not be suitable for all children, particularly those who have mild conditions.

Interestingly, it has been indicated by parents/carers who felt both positively and negatively toward the NODA system, that it might be more useful used alongside the face to face diagnostic assessment, instead of replacing it. They pointed out that not all of the behaviours and difficulties can be observed during an appointment time, just as not all of the cases can be diagnosed by relying solely on the NODA system. They also suggested that the NODA system could be used as a screening tool, to decide whether the child required additional assessments. This supports previous research that showed that diagnosticians believed that children with classic signs of ASD could be successfully and easily diagnosed by the NODA system, while further evidence such as provision of a detailed developmental history might be required for children with mild conditions, and for some of cases face to face assessment might be necessary (Nazneen, Matthews et al., 2015; Smith et al., 2017). Cranen et al.'s (2012) study also pointed out that participants considered telerehabilitation to be beneficial as a complementary, rather than an autonomous treatment (Cranen et al., 2012). Overall, the possible reasons given by parents/carers as to their perceptions toward using NODA should be taken into consideration. This study suggests that using NODA as a Store-and-Forward telehealth system is one possible way of reducing the time taken to obtain a diagnosis, although this does depend on the child's condition. It could even be used as an early screening tool alongside the in-person assessment, for those children exhibiting mild behaviours.

Generally speaking, when parents/carers were asked about their experience with the diagnostic process, most of them were dissatisfied. Factors associated with their levels of satisfaction with this were the child's age when the parents first began to have concerns about their development, the child's age when parents sought help, the child's age at diagnosis, the number of professionals consulted before receiving an ASD diagnosis, stress of the diagnostic process, degree of satisfaction with the information given to them at time of diagnosis and difficulties due to a lack of services and information. The only significant predictor of satisfaction with the overall diagnostic process was the amount of time it took to obtain a diagnosis. This finding suggests that those parents/carers who waited longer to get a diagnosis were more likely to be dissatisfied with the process than those who waited less. Clearly, more 
needs to be done to improve the experiences of parents/carers seeking a diagnosis in Wales. Decreasing the amount of time it takes to obtain a formal diagnosis would be a substantial step towards improving how parents/carers feel about the diagnostic process. The sooner they receive a diagnosis for their child, the sooner they can access services, particularly the early intervention services.

As we found in the scoping review, telehealth could be one possible way to improve access ASD diagnostic services and reduce the time taken to get a diagnosis. As we mentioned earlier, although this review suggests that telehealth is acceptable to parents, findings from that body of literature indicated that to date, no study has examined the factors informing whether or not parents of children with ASD would accept telehealth as a means of diagnostic assessment, based on a theoretical foundation. Therefore, Study (3) aimed to examine parental acceptance of using the NODA system for diagnostic assessment. This study suggests that the majority of parents/carers had a positive perception towards use of the NODA system, if it had been available before their child's diagnosis. Potential reasons were provided by most of the parents/carers who felt they either would, or would not have used the NODA system. The results also make clear that the perceived usefulness, social conditions, comfort with using laptop and mobile phone/smartphone devices as well as age, all play important role and offer a good starting point for structuring and understanding parent/carer perceptions towards the NODA system. The findings of the current study suggest that parents/carers were more likely to use the NODA system if: (1) they had a clear understanding of its benefits, such as reduced waiting time for obtaining the final diagnosis and increased chances of accessing early intervention services, (2) "important others" such as family members, friends and professionals (e.g. GP, psychologist, teacher) supported their use of the system, (3) they were more comfortable with using laptop and mobile phone/smartphone devices. At the same time, this study suggests that older parents/carers were more likely than younger parents/carers to accept the use of the NODA system for diagnostic assessment (see Figure 8-1). 


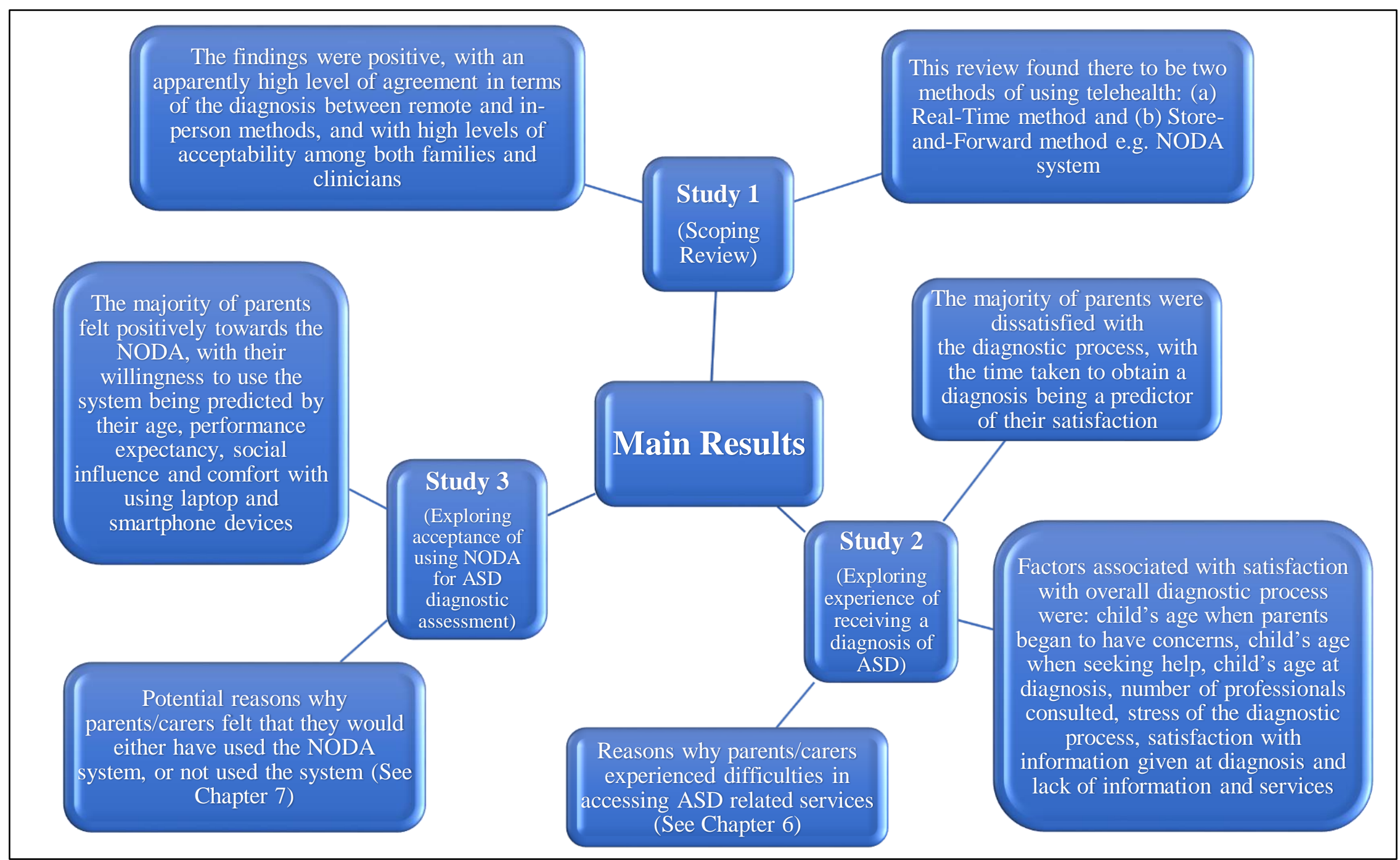

Figure 8-1: Summary of the main results. 


\subsection{Limitations}

As is the case with any research, there are some limitations to the present thesis which are briefly discussed. Firstly, although the online questionnaire was distributed electronically via several social media platforms as well as via numerous autismrelated services in Wales, the response rate was not high. As a result of this, one limitation to be aware of is that it is not possible to generalise any findings to the wider population, as they might not be applicable everywhere. Parents/carers of children with ASD are usually busy and may not have enough time to participate in research, so this may be the reason for them being unable to respond. However, significant and useful practical implications and recommendations were formulated, based on the findings from this research. Secondly, the fact that recruitment of participants and distribution of the survey was done via the Internet potentially contributes to a further unintentional limitation, because some parents of children diagnosed with ASD might not have access to the Internet, and therefore may not have the opportunity to participate in the study. Also, the findings that can be drawn from a questionnaire of this kind are reliant upon the participants who complete the survey. Investigation of respondent demographics demonstrates that the views presented in this questionnaire are largely those of mothers $(90.7 \%)$ which may cause bias. It is important for future research to sample the views of fathers regarding both the current diagnostic process and the remote diagnostic approach option. Furthermore, participants were asked to recall information which related back to when they first had concerns about the development of their child. For some participants, this might have only been a couple of years previous, but for others it might mean thinking back to more than a decade earlier. It is possible therefore, that some participants were not able to recall details accurately, which could be another limitation of the thesis.

An issue with any self-selecting sample is that the parents/carers who were unable to take part in the questionnaire may have had different perspectives. However, there was consistency across respondents' perceptions and the results therefore provide important insights both into current experiences of the ASD diagnostic process and attitudes towards using telehealth for diagnostic assessment. Also, because of time and resource limitations, the data presented in this thesis represents only partial views namely that of parents/carers which had been under-represented in previous literature. 
However, the views of other potential stakeholders, including adults and health professionals are also important and should be considered in the future research. As we stated previously that the data had some missing values, however each piece of raw data, including missing data, was reviewed and checked. It is assumed that the missing values are missing at random (MAR), and this is may be because some parents/carers did not have enough time to complete both parts of the questionnaire. Therefore, the missing values were imputed by using multiple imputation. When logistic regression analysis was conducted after imputation, in order to examine the predictors of parents/carers satisfaction with the overall diagnostic process of ASD as well as their

willingness to use NODA system for remote diagnostic assessment, the results were still the same. One final limitation that should be mentioned is that although the NODA system was introduced in the questionnaire and pictures and videos shown to the participants, they did not experience the system first hand, so may not have received a complete picture of the system, which may have affected their responses. Further research should seek to measure the actual acceptance of telehealth technology (the phase following behavioural intention) in order to analyse the conversion rate between the intention to use and actual use of this technology.

\subsection{Summary}

This chapter presents a discussion of the findings, particularly of Study (2) and Study (3), as a discussion of Study (1) is presented in Chapter 3. The key findings were explained and evaluated, showing how these relates to previous studies and research questions. This chapter also emphasised the novelty of some of the results, which appear for the first time. The discussion begins by presenting an overview of the research. Then, Study (2) findings related to the parental experience of the diagnostic process of ASD in Wales were discussed, along with the factors associated with a parent/carer satisfaction with the overall ASD diagnostic process as well as predictors of their satisfaction. This was followed by a discussion of the Study (3) findings that aimed to examine the predictors of parent/carer acceptance of using the NODA system for diagnostic assessment. To conclude, we will present the limitations of this thesis in the following chapter, provide any implications along with some recommendations for future research and concluding remarks. 


\section{Chapter 9: Conclusion}

\subsection{Introduction}

This chapter concludes the thesis by highlighting its limitations. It then provides some theoretical and practical implications. Finally, it offers some recommendations for future research and concluding remarks.

\subsection{Implications}

The various studies within this thesis have a variety of implications. From a theoretical perspective, the scoping review about the use of telehealth for facilitating the diagnostic assessment of ASD (Study 1) builds a foundation for inquiry into the telehealth approaches that are used in the diagnosis and assessment of ASD, and gives a scope as to whether they are feasible and acceptable, and how they compare with inperson diagnosis and assessment methods. Furthermore, the Study (2) results may assist in understanding the experiences of parents of children with ASD seeking to obtain a diagnosis of ASD in Wales, and contribute to identifying the factors associated with the satisfaction of parents/carers with the overall diagnostic process. This thesis has also explored the knowledge of the factors influencing acceptance of the NODA system in Wales (Study 3). The key theoretical contribution of this thesis is the development of the conceptual framework of acceptance of the NODA system, using the UTAUT model. The variables identified within this research can be used in future studies examining the adoption of other telehealth diagnostic technologies.

The thesis also has several practical implications for those working within the health service delivery system in Wales. The Study (2) suggests that professionals should be more considerate of the parents/carers early concerns about their child's development. It is imperative to ensure that primary healthcare providers, such as GP and health visitors, are knowledgeable about the signs of ASD and refer children for assessment when required. Increasing their access to adequate training may be necessary. Efforts should also be made to raise the general awareness of parent/carers and improve their knowledge about child development, including early signs of ASD, as that would help to obtain earlier diagnosis and interventions. This study suggests that parents/carers in 
Wales experience high levels of stress during the diagnostic process for ASD. Professionals need to be aware of the impact of this process on parents and families, in order to be able to provide appropriate help and support. This should begin with discussing parents/carers concerns about the development of their child until a confirmed diagnosis is received, and even post diagnosis. Furthermore, consistent with the previous research (Mansell \& Morris, 2004), an early diagnosis might be a main contributory factor in reducing stress associated with the diagnostic process for parents/carers. This may, in turn, improve their experiences of and perceptions towards the diagnostic process as a whole. This research also highlights the importance of providing parents with general information on the ASD diagnosis and the effects of early interventions, based on available evidence. Resources such as written lists of interventions and behavioural management strategies are crucial information that parents/carers may well find useful.

Furthermore, the findings showed that the greater number of professionals that parents/carers saw as they sought to secure a diagnosis, the more negatively they viewed the diagnostic experience. More professional visits might mean more waiting time, which could possibly increase levels of stress and frustration for parents/carers. Around half of parents reported that they had discussed their concerns with five or more professionals before obtaining a diagnosis. It is therefore recommended that the number of professionals needed to evaluate a child be kept to a minimum, which this may improve parental satisfaction with the diagnostic process. The results of this study also indicate that parents/carers may experience of a lack of necessary services for their children and a shortage of professionals in Wales. This may effect the waiting time for accessing services e.g. diagnostic assessment. Some families may have to travel long distances or otherwise attend private clinics in order to obtain a diagnosis. This underlines the need to increase the supply of providers who are working in the field of autism in Wales.

One of the major findings of this thesis was that on average, parents/carers face a long delay of around 3.9 years between first seeking help and obtaining a confirmed diagnosis of ASD for their child. This time taken to receive a diagnosis was also a predictor of the parents/carers satisfaction with the overall diagnostic process. The long waiting times to receive a final diagnosis might cause stress for the parents/carers 
and their families (Mansell \& Morris, 2004), which could in turn increase their exposure to anxiety and depression (Hayes \& Watson, 2013; Siklos \& Kerns, 2007). Delay in diagnosis may also result in a lack of early intervention services, which are critical for securing positive outcomes (Matson, 2008; McEachin et al., 1993; Rattazzi, 2014; Siegel, 2008; Smith et al., 2017). Evidently, more needs to be done to improve their experience of the diagnostic process for parents/carers in Wales. Reducing the time taken to obtain a diagnosis would be a considerable step forward in improving the diagnostic process and therefore the attitudes of parents/carers toward the process. The sooner parents/carers receive a diagnosis for their child, the sooner they can access services, particularly early intervention services. Parents/carers feelings might also be relieved if they receive early explanations for their child's problems (Howlin \& Moore, 1997; Mansell \& Morris, 2004).

Telehealth represents a potential solution that may improve access for parents/carers to ASD diagnostic services and help in reducing the time taken to get a diagnosis in Wales. Use of telehealth could also help in expanding diagnostic services rapidly to a greater population, to enable early detection and assessment, especially in the rural areas (Oberleitner et al., 2007; Reese, Braun et al., 2015). However, user (e.g. parent/carer) acceptance is considered to be an essential contributory factor for the success of any information system schemes, including telehealth (Davis, 1993; Dillon \& Morris, 1996; Hu et al., 1999). Highlighting what may influence whether parents/carers accept the remote diagnostic approach could help to predict whether it will be adopted in the future.

This thesis identified the predictors that might improve the chances that parents/carers of children with ASD in Wales would accept NODA as a telehealth system (Study 3). Therefore, health care organisations planning to develop a Store-and-Forward telehealth diagnostic ASD system such as this are advised to consider the predictors found in this study. Effort expectancy was found to be one of the significant determinants of willingness to use the NODA system. This implies that the potential benefits of such a system (e.g. decreased waiting time to obtain diagnosis and increased chances of accessing early intervention services) should be clearly presented to parents/carers. Social influence is another predictor that health care organisations should consider when introducing new telehealth ASD diagnostic system. 
Parents/carers were influenced by family members, friends and professionals (such as paediatricians, speech and language therapists, psychiatrists, occupational therapists) who may recommend using the technology. This indicates that if professionals took the lead in using the technology for remote ASD diagnosis, parents/carers might be expected to also use the system. Moreover, the age of parents/carers had a significant influence on whether or not they would accept NODA. Thus, telehealth services aiming to support diagnostic assessment of ASD, such as the NODA system, should be designed taking into considerations the age of all possible users. Comfort with using mobile phones/smartphones and laptops were also predictors of acceptance of the NODA system. This suggests that, when introducing a new telehealth ASD diagnostic system, health care organisations should provide adequate knowledge and instructions, clearly explaining the basic functions of the electronic devices such as mobile phones/smartphones and laptop computers. This may help parents/carers to improve their familiarity with devices, which in turn might improve the chances of them accepting the use of such telehealth systems for ASD diagnostic assessment.

This study also provided some potential reasons regarding the perceptions of parents/carers towards using the NODA system, that should be taken in the consideration. One of the main findings showed that parents/carers felt that NODA could compliment in-person diagnosis, instead of replacing it. Therefore, any future Welsh initiatives ought to consider using the telehealth ASD diagnostic approach in addition to the face to face diagnostic assessment. Telehealth alone could be sufficient to diagnose children with classic ASD symptoms, while for complicated cases, inperson diagnosis might be needed for a more comprehensive assessment. In such cases however, telehealth diagnostic methods would still be very useful as an early screener. As Wales has large rural areas (The Welsh NHS Confederation, 2018), the findings of this study suggest that the telehealth diagnostic approach could be used to connect isolated families with diagnosticians, in order for them to obtain a diagnosis. Even in more urban areas, it could also be used to triage cases on current waiting lists for diagnostic assessments, separating clear-cut cases from those children who will definitely require a face to face diagnostic assessment. 
The current COVID-19 crisis has meant that the NHS has been forced to rethink the delivery of services and has brought to the fore the ways in which technology, in particular on-line consultations and other telehealth tools, can be successfully used to deliver health services. These recent changes brought in by COVID-19 could also pave the way for an overhaul in the ways that ASD is diagnosed, to include the use of telehealth such as the NODA system.

\subsection{Recommendations for future research}

This section highlights some recommendations for future research, based on the main findings of this thesis, any limitations as well as areas worthy of additional consideration. One of the goals of remote diagnostic system is to reduce the time between parents raising concerns and receiving a diagnosis. Therefore, researchers may consider conducting randomised controlled trials to confirm that telehealth can improve the time taken to get an ASD diagnosis.

Since this research used a quantitative method to study the acceptance of telehealth (NODA system) for remote ASD diagnosis, future studies could consider expanding this method. In other words, the quantitative findings of this research could be investigated further, using qualitative methods such as interviews and/or focus groups. This would bring further understanding around the willingness to adopt and use the NODA system. The quantitative findings of exploring the parent/carer experience in obtaining an ASD diagnosis could also be further examined by using qualitative methods, as this may provide a "voice" for parent/carer concerns, which may in turn reveal more pertinent information. However, some open-ended questions were already used in both studies, to help participants elaborate their answers that were provided within closed questions. Furthermore, as explained previously, this research had a low response rate. Future studies should be conducted with larger samples, to allow for results to be generalised to other populations.

In terms of research sites, as this research only surveyed parents/carers of children with ASD in Wales, any future directions could include investigating other parts of the UK, such as England and Scotland. The research could be conducted in another part of the world, either in developed or developing countries. It would be particularly important 
to conduct such research in countries or cities where access to healthcare services (e.g. ASD diagnostic related services) is limited and difficult. In terms of telehealth technology, whereas this study examined the acceptance of Store-and-Forward technology (NODA system), a further study could consider studying the attitudes of parents of children with ASD towards using Real-Time technology for remote diagnostic assessment e.g. videoconferencing.

As mentioned previously in Chapter 3, user acceptance is considered as the essential factor to determine either the success or failure of new information systems, including telehealth (Davis, 1993; Hu et al., 1999). Therefore, it is recommended that future studies be conducted with other populations, including adults and health professionals (e.g. paediatricians, psychiatrists, occupational therapists, speech and language therapists, psychologists) so as to build up a comprehensive picture on the subject of the acceptance of using telehealth for remote ASD diagnosis.

Although the model was able to explain between $38.4 \%$ and $53.2 \%$ of the variance in acceptance, which is considered reasonable, particularly as a first step towards understanding the adoption of telehealth technology for ASD diagnosis in Wales, it is recommended that researchers investigate behavioural intention by extending the UTAUT model with other theoretical variables. Despite the fact that the UTAUT is a robust model, integrating a number of acceptance models and theories, future studies could also consider using other theoretical frameworks. Uncovering new factors may help in gaining more knowledge about the acceptance of using telehealth technology for remote diagnosis.

\subsection{Concluding remarks}

Overall, this thesis has fulfilled its overarching aim to examine whether telehealth technology can be used (the degree of acceptance by parents/carers) to increase access to ASD diagnostic services in Wales, UK and reduce the time taken to receive a diagnosis. Results of Study (2) indicated that most parents/ carers were dissatisfied with the diagnostic process of ASD in Wales. The time taken to obtain a confirmed ASD diagnosis was found to play a key role in the level of their satisfaction with such a process. Study (1) has provided early evidence about the use of telehealth for ASD 
diagnosis, that suggests that the systems available to date are feasible and acceptable to the families. Such systems also have the potential to improve the time required for diagnosis of ASD. Study (3) contributed to an understanding of how parents/carers felt about accepting the NODA system for remote ASD diagnostic purposes. Results indicate that most of the respondents had a positive perception towards NODA. Perceived usefulness, social influence, age of parent/carer and comfort with using laptop and mobile phone/smartphone devices were the predictors which impacted upon this decision.

Finally, it is necessary to reiterate that children with ASD do not live in isolation, but rather, within a family system. The lengthy delay in receiving a diagnosis has implications for both children and their families. It might impact upon the long-term development of a child, if the window of opportunity for the early interventions is missed. The long waiting times to receive a final diagnosis can also cause stress for families (Crane et al., 2015; Hayes \& Watson, 2013; Zwaigenbaum et al., 2016). This research supported this assertion, by identifying that the majority of parents/carers felt that the diagnostic process was very stressful. Therefore, use of telehealth approaches for remote ASD diagnosis might be one of the potential solutions for improving the process and reducing the overall time taken to obtain a final diagnosis. This may, in turn, make the parental experience more satisfactory. This research could be a useful starting point to understand what influences the acceptance of using the telehealth approach (e.g. NODA system) for ASD remote diagnostic assessment among parents/carers in Wales and, in doing so, has paved the way for future developments.

\subsection{Summary}

This chapter concludes the thesis by briefly describing its limitations. It also presented some theoretical and practical implications. Finally, it provided some suggestions for future research directions and concluding remarks. 


\title{
Appendices
}

\author{
Appendix 1: Research Article: Use of Telehealth for Facilitating the Diagnostic \\ Assessment of Autism Spectrum Disorder (ASD): A scoping Review
}

\section{PLOS ONE}

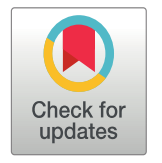

\section{G OPENACCESS}

Citation: Alfuraydan M, Croxall J, Hurt L, Kerr M, Brophy S (2020) Use of telehealth for facilitating the diagnostic assessment of Autism Spectrum Disorder (ASD): A scoping review. PLOS ONE 15 (7): e0236415. https://doi.org/10.1371/journal. pone. 0236415

Editor: Amanda A. Webster, University of Wollongong, AUSTRALIA

Received: January 6, 2020

Accepted: July 6, 2020

Published: July 23, 2020

Copyright: $\odot 2020$ Alfuraydan et al. This is an open access article distributed under the terms of the Creative Commons Attribution License, which permits unrestricted use, distribution, and reproduction in any medium, provided the original author and source are credited

Data Availability Statement: All relevant data are within the manuscript and its Supporting Information files.

Funding: This study was funded by Saudi Arabia Cultural Bureau in the UK. The funders had no role in study design, data collection and analysis, decision to publish, or preparation of the manuscript.

Competing interests: The authors have declared that no competing interests exist.
RESEARCH ARTICLE

\section{Use of telehealth for facilitating the diagnostic assessment of Autism Spectrum Disorder (ASD): A scoping review}

Manahil Alfuraydan $\bigodot^{1,2 *}$, Jodie Croxall ${ }^{1}$, Lisa Hurt ${ }^{3}$, Mike Kerr $^{4}$, Sinead Brophy ${ }^{5}$

1 School of Medicine, Swansea University, Swansea, United Kingdom, 2 College of Applied Medical Sciences, King Faisal University, Hofuf, Saudi Arabia, 3 Division of Population Medicine, School of Medicine, Cardiff University, Cardiff, United Kingdom, 4 Division of Psychological Medicine and Clinical Neuroscience, Cardiff University, Cardiff, United Kingdom, 5 National Centre for Population Health and Wellbeing, School of Medicine, Swansea University, Swansea, United Kingdom

*842703@swansea.ac.uk

\section{Abstract}

There is a significant delay between seeking help and a confirmed diagnosis of Autism Spectrum Disorder (ASD). This delay can lead to poor outcomes for both the families and individuals. Telehealth potentially offers a way of improving the diagnostic pathway for ASD. We conducted a scoping review examining which telehealth approaches are used in the diagnosis and assessment of ASD in children and adults, whether they are feasible and acceptable, and how they compare with face-to-face diagnosis and assessment methods. A search for all peer-reviewed articles, combining the terms of autism and telehealth was conducted from 2000 to 2019. A total of 10 studies were identified for inclusion in the review. This review of the literature found there to be two methods of using telehealth: (a) Real-Time method e.g. video conferencing that enables teams in different areas to consult with the families and to assess the child/adult in real time and (b) A Store-and-Forward method as Naturalistic Observation Diagnostic Assessment (NODA) system to upload videos of child's behaviors to a webportal that enables the clinicians to make an assessment remotely. The findings were positive, finding there to be high agreement in terms of the diagnosis between remote methods and face to face methods and with high levels of satisfaction among the families and clinicians. This field is in the very early stages and so only studies with small sample size using surveys and interviews were identified but the findings suggest that there is potential for telehealth methods to improve access to assessment and diagnosis of ASD used in conjunction with existing methods, especially for those with clear autism traits and adults with ASD. Larger randomised controlled trials of this technology are warranted.

\section{Introduction}

Autism Spectrum Disorder (ASD) is a developmental condition characterised by impairment in terms of social communication and social interaction and a repetitive and restricted pattern of interest, behaviour and activity [1]. Around 1 in 54 children in the United States has been 
diagnosed with ASD [2]. In respect of the diagnostic criteria, the latest revision of the Diagnostic and Statistical Manual of Mental Disorders "DSM-5", published in May 2013, approved the umbrella term ASD without any subtypes. It organised the impairments into two domains: difficulties in social communication and social interaction; and restricted and repetitive behaviour, interests, or activities [1]. The diagnosis of ASD may include physical examinations to rule out physical causes of difficulties, speech and language assessment, observation, and a history of the child's development and behaviour, focusing on features consistent with the DSM5 criteria [3, 4]. Commonly used tools to aid diagnosis include the Autism Diagnostic Interview-Revised (ADI-R) and Autism Diagnostic Observation Schedule (ADOS-2) [4]. The $\mathrm{ADI}-\mathrm{R}$ is a semi-structured interview that is conducted by clinician with a parent/carer who is familiar with developmental history as well as child' current behaviour [5]. The ADOS-2 has 5 modules which involve 40-60 minute protocols of activities that are based on playing, communication and social interactions [6].

Research reports a delay of approximately 20 months to 5 years between seeking help and a confirmed diagnosis of ASD [7-10]. This may result in delayed access to early intervention services, which are critical for positive outcomes [11-15]. The long wait times also cause stress to individuals and families $[7,16]$. Some of the reasons for the delays in diagnosis include the shortage of healthcare professionals [17] and several specialist and treatment appointments are often needed to make a diagnosis. Multiple meetings with a variety of professionals in different locations can also be very stressful for individuals who might later be diagnosed with ASD. This is as people with ASD are very sensitive to changes in their situations and excessively reliant on routines [1]. Some families have to travel long distances to access these services [18]. This is not only a cost to the family but also to the specialist healthcare teams who travel to reach different areas. Recent research found that healthcare and educational systems work independent from each other with little crossover of activity [19], therefore it may be difficult to get multi-disciplinary teams together, especially when they span different disciplines.

Overall, there seems to be some evidence to indicate that parents of children with ASD and adults face difficulties in getting ASD diagnosis. Therefore, more information is needed as to how to improve diagnostic services to address the well-being of the child and improve the experiences of the parents and adults with ASD. Telehealth can provide help and support to populations with specific health and well-being needs. Telehealth is identified as a "mechanism that enables individuals to receive professional services and support at a distance" [20 p.2953]. This may involve a Real-Time or Store-and-Forward methods. Real-Time interaction allows patient to communicate in real time with a health care provider. Videoconferencing is viewed a main form of Real-Time communication for telehealth programs. While, Store-and-Forward interaction does not depend on the concurrent presence of parties (e.g. healthcare provider and patient) [21].

Telehealth may have advantages over traditional face-to-face approaches, including improving access to healthcare services especially for individuals and families living in rural and underserved areas [22]. There is growing evidence to suggest that telehealth approaches could decrease providers' and patients' costs (e.g. travel time, transportation expenses, missed work) and increase coverage area to providers $[23,24,25]$. Users of such programs can also interact directly with clinicians as well as with instructional content via video, email and apps systems $[26,27]$. By offering families with a chance to play an important role in the child's development, telehealth technology could expedite diagnostic process and early intervention services $[26,28]$. However, limited access to the required technologies such as computers and mobile phones could hamper the widespread use of telehealth [29]. In prior telehealth evaluations for children with ASD, parents required good internet access [20, 26, 30]. Such access could be challenging for some communities. Especially, individuals in rural and underserved 
areas may be disadvantaged by lack of mobile coverage [31]. Slow internet speed, poor quality and unreliable connectivity problems may result in frustration and unwillingness to use telehealth approaches among families and adults with ASD [29]. Some families have concerns that children may change their behaviours if they know they are observed distantly [28, 32]. A further challenge to the use of telehealth seems to be lack of skills and confidence in using technology among some individuals [31]. Reluctance of service providers is also considered as one of the barriers. Limited experience with remote communication systems and concerns about interactions with adults, children and their families have been revealed as potential reasons for their reluctance $[32,33]$.

The previous literature reported that telehealth is currently used by numerous clinical applications with positive results, such as tele-radiology [34], tele-cardiology [35], and looking into the condition of home-based patients with diabetes and hypertension [21] as well as tele-mental health services [36]. Prior studies also showed that telehealth may be a promising means to facilitate providing a diagnosis in different conditions. For instance, in establishing a diagnosis of dementia, it has been found that telehealth was as accurate as a face to face clinical examination with a high degree of satisfaction correlated with using telehealth [37]. The research also demonstrates that using telemedicine for a congenital heart disease diagnosis is both accurate and safe [38]. Furthermore, telehealth using slit lamp images was suggested to be reliable and accurate method for diagnosis eye problems in accident and emergency departments [39].

Because of the benefits these systems deliver, the notion was moved to the world of ASD [40]. The position of telehealth in the field of ASD is small but growing. Telehealth approaches have recently been explored as a way of supporting the delivery of a range of services for people with ASD and their families. Telehealth might be used to improve individuals' access to behavioural intervention services. Several studies used telehealth to coach parents of children with ASD in order to conduct behavioural assessments such as functional analyses (FA), functional communication training (FCT) via the use of videoconferencing, either parents and their child located at regional clinic, home or school [41-47]. The findings suggested that parents can successfully conduct FA and FCT through telehealth when behaviour analyst offer consultation remotely. It has also been found reductions in problem behaviour of children. For example, Wacker et al. [46] found the average reduction in problem behaviour was $93.5 \%$. Similarly, Lindgren et al. [41] revealed that problem behaviour was decreased by an average of $90 \%$. It has also been demonstrated the preliminary efficacy of telehealth-delivered parent mediated interventions that are intended to increase parent knowledge and the use of ASD behavioural intervention strategies with the child in their daily life. Several studies suggested that use of telehealth program, combining web-based instructional content with weekly video-conferencing coaching sessions may support parental learning and improve child's social communication skills [20, 26, 30, 48, 49]. Parents also indicated that such systems were effective, acceptable and useable.

Findings from these studies provide initial evidence for the feasibility, acceptability and effectiveness of telehealth technologies to serve as models for delivering parental training to support conducting the behavioural interventions and helping parents understand and use intervention practices in their daily interaction with children. Given this success, it is possible that telehealth may also be useful in improving the diagnostic pathway of ASD.

So far, very limited research has been conducted in the literature regarding the use of telehealth in ASD. For example, a review by Boisvert et al. [50] included only the studies that used telepractice directly to individuals with ASD, but did not include studies that involved others such as parents of children with ASD. Other previous reviews [51,52] have focused on studies that centred around the use of telehealth and Internet-based interventions for parental or caregiver training purposes. However, no previous literature review has examined specifically the use of telehealth to support ASD diagnostic assessment. Our review aims to fill this gap and 
extends prior work by providing a scoping review to examine which telehealth approaches have been used in the diagnosis and assessment of ASD in children and adults, whether they are feasible and acceptable, and how they compare with face-to-face diagnosis and assessment methods. A scoping review was conducted to broadly find out what is known about using telehealth for ASD diagnostic assessment. Contrary to a systematic review which mainly focuses on identifying and appraising the quality of the evidence that is relevant to a particular question/questions [53], a scoping review investigates an area of literature more broadly regardless of methodological quality with intention of examining the extent, nature, and range of research activity $[53,54]$.

\section{Method}

The PRISMA extension for scoping reviews (PRISMA-ScR) checklist was used to ascertain the key items to report for a scoping review (see S1 Table) [55]. The scoping review was not registred previously.

\section{Search strategy}

The following electronic bibliographic databases were used: MEDLINE, CINAHL Plus with Full text, Business Sources Complete, Web of Science, Scopus and PsycINFO and trail and systematic review databases including Cochrane Library, Health Technology Assessment, Database of Abstracts and Reviews of Effectiveness and NHS Economic Evaluation. Online sources searched included guidelines, government health sites, and ASD related associations. Searches were limited to the English Language from January 2000 to December 2019 inclusive.

The rationale for not including studies prior to 2000 is that telehealth technology was not involved in the ASD field until 2004 [40], however the literature search was conducted from January 2000 to make sure that there is no study that could be consistent with our inclusion criteria. The terms for autism were combined with terms for telehealth to identify eligible studies (see Box 1 an example of a full search in Medline).

\section{Inclusion and exclusion criteria}

Inclusion criteria specified that studies should involve telehealth technology and should report outcomes (not merely describe a service). We define the telehealth technology as any technology that provides ASD diagnostic assessment services at a distance. In respect of the outcomes, as this review is comprehensive, we are considering all of the outcomes that are reported when telehealth has been used in the diagnosis and assessment of ASD in children and adults, e.g. the feasibility and acceptability of telehealth approaches in the diagnosis and assessment of ASD, as well as the accuracy of remote diagnosis compared with in-person methods. As this is a developing field, we included all study designs to fully understand the current state of the literature. Only primary studies were included. The study population was anyone going through an ASD diagnosis process with any kind of healthcare practitioner. Any study that considered only technical issues, editorials or opinions or those concerned with medical education were excluded. In studies of children (younger than 18 years old), information was usually reported by parents/carers, and in studies of adults ( 18 years old and older), the information could be given by the adults themselves or their carers.

\section{Data extraction and synthesis}

Articles, abstracts or titles identified from the search strategy were reviewed by MA to determine if they met the inclusion criteria. In cases where the eligibility of the study was unclear, it 
Box 1. Example of search strategy used in MEDLINE database.

"Autism Spectrum Disorder" OR "Autistic Disorder" OR autis* OR asperger* OR ASD OR neurodevelopmental disabilit" OR developmental disorder* OR Pervasive developmental disorder

AND

telemedicine OR telehealth OR telemonitoring OR ehealth OR mhealth OR m-health OR Telepractice OR Teletherapy OR Telecare OR Telediagnostic OR Teleconference OR Teleassessment OR videoconf* OR mobile tech* OR mobile app* OR web portal OR internet OR web-based OR web-delivered OR Web conference OR online OR Skypeß OR iChat $\beta$

AND

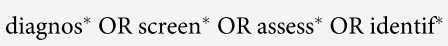

Limits: from January 2000 to December 2019, English Language.

Results: 774 studies

was reviewed by a second reviewer (SB or JC). The full texts were retrieved and reviewed in full by two independent researchers (MA, SB or JC). There was no disagreement in terms of agreement and a third person did not need to adjudicate. Author, year of publication, country, participant characteristics, study design, technology utilised, services delivered, and outcomes were extracted for each paper using a standard form in Excel.

\section{Quality assessment}

Independent assessment of the methodological quality was conducted by two authors (MA, SB or JC) for included studies. In case of differences, consensus was reached by discussion or through consulting (SB or JC). The National Heart, Lung, and Blood Institute (NHLBI) Quality Assessment for Observational Cohort and Cross-Sectional Studies tool [56] was used for five studies $[9,59,60,61,63]$. The items of the tool were evaluated by using "yes", "no", "not applicable", "cannot determine", or "not reported". This was used to guide the overall rating for the quality of each study as "good", "fair", or "poor". The Diagnostic research Critical Appraisal Skills Program (CASP) tool [57] was also used for four studies [15, 22, 58, 62] and Qualitative research Critical Appraisal Skills Program (CASP) tool [57] for one study [28]. The items of the tools were assessed by using "yes", "no", or "cannot tell". As there are no quality scores in CASP tool, we have decided to use the same quality scores of NHILBI quality assessment tools ("good", "fair", or "poor") [56] for each study.

\section{Results}

\section{Study characteristics}

A total of 3698 articles were identified using the search strategy, of which 124 were selected for abstract screening based on title screening. Of these, 37 were selected for full text review and 10 papers were selected for inclusion in the review and data extraction (Fig 1). The studies that were rejected had mainly examined treatment of ASD using telehealth rather than diagnosis. 


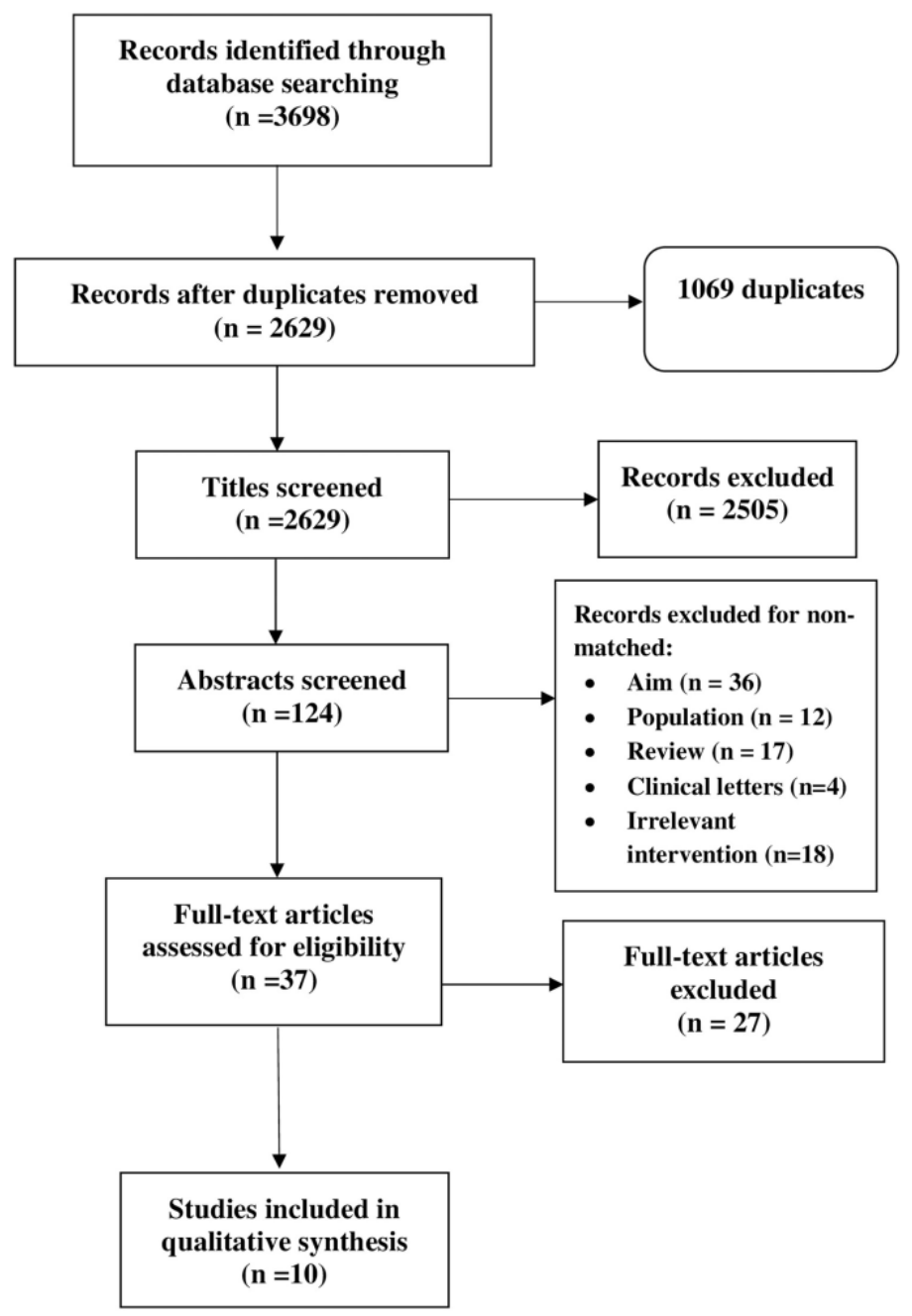

Fig 1. Flowchart of study selection process.

https://doi.org/10.1371/journal.pone.0236415.g001

In addition, studies that were reviews, clinical letters, or including a non ASD population or not using telehealth technologies were rejected. All 10 studies were based in the USA and published between 2013 and 2018 (Table 1). There were 5 independent research teams as 3 papers were by Reese et al. [22, 58, 59], 2 were by Nazeen et al. [9, 28], 2 were with Parmanto and team $[60,61], 2$ were with Juarez and team $[62,63]$ and one study was by Smith et al. [15]. A 
Table 1. Summary of study characteristics.

\begin{tabular}{|c|c|c|c|c|c|c|}
\hline Author, year & Country & Participants Characteristics & Study design & Technology utilized & Service delivered & Outcomes \\
\hline $\begin{array}{l}\text { Nazneen } \\
\text { et al., } 2015 \\
{[28]}\end{array}$ & USA & $\begin{array}{l}43 \text { parents, } 51 \text { target children } \\
\text { and their siblings, and } 10 \\
\text { diagnosticians. Children were } \\
\text { between the ages of } 18 \text { months } \\
\text { and } 6 \text { years, } 11 \text { months. }\end{array}$ & $\begin{array}{l}\text { Qualitative study } \\
\text { Interview }\end{array}$ & NODA & $\begin{array}{l}\text { Remote ASD } \\
\text { diagnosis }\end{array}$ & $\begin{array}{l}\text { Parents and diagnosticians } \\
\text { found that NODA is easy to use } \\
\text { and effective approach to } \\
\text { address the obstacles to autism } \\
\text { diagnosis. They reported } \\
\text { possible barriers such as privacy } \\
\text { concerns and reactivity of } \\
\text { behavior. }\end{array}$ \\
\hline $\begin{array}{l}\text { Nazneen } \\
\text { et al., } 2015 \\
{[9]}\end{array}$ & USA & $\begin{array}{l}\text { Stage 1: one-on-one } \\
\text { interview: } 7 \text { parents of } \\
\text { children with autism and } 11 \\
\text { clinicians. Stage } 2: \text { iterative } \\
\text { development: } 8 \text { families, } 18 \\
\text { children and their siblings } \\
\text { Stage3: in-field evaluation: } 4 \\
\text { families with at least } 1 \text { child } \\
\text { with previous ASD diagnosis } \\
\text { and } 1 \text { family with a typically } \\
\text { developing child and } 2 \\
\text { diagnosticians. Children were } \\
\text { between } 2 \text { and } 6 \text { years of age. }\end{array}$ & $\begin{array}{l}\text { Pilot study } \\
\text { Interview, } \\
\text { development, and } \\
\text { evalution. }\end{array}$ & NODA & $\begin{array}{l}\text { Remote ASD } \\
\text { diagnosis }\end{array}$ & $\begin{array}{l}\text { Parents can easily record videos } \\
\text { of their children behaviors. The } \\
\text { clinical validity ratings of the } \\
\text { collected videos were } 96 \% \text {. For } 4 \\
\text { out of } 5 \text { children were remotely } \\
\text { diagnosed by both } \\
\text { diagnosticians that matched } \\
\text { with the child's actual diagnosis. }\end{array}$ \\
\hline $\begin{array}{l}\text { Smith et al., } \\
2016[15]\end{array}$ & USA & $\begin{array}{l}40 \text { families seeking an ASD } \\
\text { assessment for their children } \\
\text { and } 11 \text { families of typically } \\
\text { developing children. Children } \\
\text { were between the ages of } 18 \\
\text { months and } 6 \text { years } 11 \text { months }\end{array}$ & $\begin{array}{l}\text { Diagnostic } \\
\text { accuracy study (In- } \\
\text { person assessment/ } \\
\text { NODA) }\end{array}$ & NODA & $\begin{array}{l}\text { ASD remote } \\
\text { diagnosis }\end{array}$ & $\begin{array}{l}\text { There was substantial } \\
\text { agreement between NODA and } \\
\text { in-person assessment (IPA) }\end{array}$ \\
\hline $\begin{array}{l}\text { Reese et al., } \\
2013 \text { [58] }\end{array}$ & USA & $\begin{array}{l}11 \text { children with autism and } 10 \\
\text { with an existing diagnosis of } \\
\text { developmental delay }(n=21) \\
\text { Ages: } 3-5 \text { years old. }\end{array}$ & $\begin{array}{l}\text { Pilot study } \\
\text { Diagnostic } \\
\text { accuracy study }\end{array}$ & Video- conferencing & \begin{tabular}{|l|} 
Conducting ASD \\
assessment (ADOS- \\
2 and ADI-R) \\
Diagnosis
\end{tabular} & $\begin{array}{l}\text { No significant difference in the } \\
\text { diagnostic accuracy, ratings for } \\
\text { ADI-R, ADOS observations, } \\
\text { and parent satisfaction between } \\
\text { IVC and InP conditions. }\end{array}$ \\
\hline $\begin{array}{l}\text { Reese, et al., } \\
2015[22]\end{array}$ & USA & $\begin{array}{l}17 \text { families of young children } \\
\text { requesting an evaluation for } \\
\text { ASD. Ages:2.5-6 years old. }\end{array}$ & $\begin{array}{l}\text { Diagnostic } \\
\text { accuracy study }\end{array}$ & Video-conferencing & \begin{tabular}{|l|} 
Conducting ASD \\
assessment (ADOS- \\
2 and ADI-R) \\
Diagnosis
\end{tabular} & $\begin{array}{l}\text { Results revealed excellent } \\
\text { interrater agreement on } \\
\text { diagnoses between clinicians in } \\
\text { the VC setting and } \\
\text { interdisciplinary assessment } \\
\text { team. }\end{array}$ \\
\hline $\begin{array}{l}\text { Reese, et al., } \\
2015 \text { [59] }\end{array}$ & USA & 370 families & Retrospective study & ISUT & $\begin{array}{l}\text { Examining } \\
\text { telehealth's impact } \\
\text { on families' access } \\
\text { to diagnostic } \\
\text { services }\end{array}$ & $\begin{array}{l}\text { ISUT provided families } \\
\text { increased access to diagnostic } \\
\text { services. }\end{array}$ \\
\hline $\begin{array}{l}\text { Parmanto } \\
\text { et al., 2013 } \\
\text { [60] }\end{array}$ & USA & $\begin{array}{l}5 \text { clinicians } 10 \text { adults with } \\
\text { disability Age: } 17 \text { years old }\end{array}$ & Case study & Videoconferencing & $\begin{array}{l}\text { Remote assessment } \\
\text { of adults with ASD } \\
\text { (ADOS Module 4) }\end{array}$ & $\begin{array}{l}\text { The patients were satisfied with } \\
\text { the developed telehealth system } \\
\text { and provided feedback that it } \\
\text { was easy to use }\end{array}$ \\
\hline $\begin{array}{l}\text { (Schutte } \\
\text { et al., 2015) } \\
{[61]}\end{array}$ & USA & $\begin{array}{l}23 \text { adults with an ASD } \\
\text { diagnosis. Ages: } 22 \text { years old }\end{array}$ & $\begin{array}{l}\text { Within-subject } \\
\text { crossover study. } \\
\text { Questionnaire. }\end{array}$ & $\begin{array}{l}\text { Versatile and Integrated } \\
\text { System for Tele- } \\
\text { rehabilitation (VISYTER). It } \\
\text { consists of } \\
\text { videoconferencing and web } \\
\text { portal. }\end{array}$ & $\begin{array}{l}\text { Remote assessment } \\
\text { of adults with ASD } \\
\text { (ADOS Module 4) }\end{array}$ & $\begin{array}{l}\text { Participant satisfaction was } \\
\text { high. It demonstrated that an } \\
\text { adult autism assessment can be } \\
\text { administered distantly with high } \\
\text { levels of reliability. }\end{array}$ \\
\hline
\end{tabular}

(Continued) 
Table 1. (Continued)

\begin{tabular}{l|l|l|l|l|l|l}
\hline Author, year & Country & Participants Characteristics & Study design & Technology utilized & Service delivered & Outcomes \\
\hline $\begin{array}{l}\text { Juarez et al., } \\
\text { 2018 [62] }\end{array}$ & USA & $\begin{array}{l}\text { Study1: } 20 \text { children and their } \\
\text { caregivers (16 boys, } 4 \text { girls) } \\
\text { Ages: between 20 and 34 } \\
\text { months. Study2: } 45 \text { children } \\
\text { and their caregivers (35 boys, } \\
10 \text { girls) Ages: between 19 and } \\
\text { 32 months. }\end{array}$ & $\begin{array}{l}\text { Study1: Diagnostic } \\
\text { accuracy study } \\
\text { Study2: Feasibility } \\
\text { study }\end{array}$ & Video-conferencing & $\begin{array}{l}\text { ASD remote } \\
\text { diagnosis }\end{array}$ & $\begin{array}{l}\text { Study1: all of the children } \\
\text { (n=15) who received ASD } \\
\text { diagnosis via telehealth were } \\
\text { confirmed by face to face } \\
\text { assessment. Study2: clinicians } \\
\text { were satisfied with the } \\
\text { telemedicine in } 80 \% \text { of cases } \\
\text { (n=36) but would rather to } \\
\text { assess a child in person 24\% of } \\
\text { the time (n=11). }\end{array}$ \\
\hline $\begin{array}{l}\text { Stainbrook } \\
\text { et al., 2018 } \\
\text { [63] }\end{array}$ & USA & 63 families & Retrospective study & Video-conferencing & $\begin{array}{l}\text { Measuring } \\
\text { Telediagnosis } \\
\text { impact on referrals }\end{array}$ & $\begin{array}{l}\text { Telemedicine diagnostic } \\
\text { consultation may positively } \\
\text { impact referrals for diagnostic } \\
\text { evaluation }\end{array}$ \\
\hline
\end{tabular}

ASD = Autism Spectrum Disorder, USA = United States of America, NODA = Naturalistic Observation Diagnostic Assessment, ADOS = Autism Diagnostic Observation Schedule, ADOS-2 = Autism Diagnostic Observation Schedule, Second Edition, ADI-R = Autism Diagnostic Interview-Revised, IVC = Interactive Videoconferencing, ISUT = Integrated System Using Telemedicine, VISYTER = Versatile and Integrated System for Tele-rehabilitation .

https://doi.org/10.1371/journal.pone.0236415.t001

total of 997 people participated across the 10 papers and of these 629 were parents, 291 were children (with ASD) 33 were adults (with ASD) and 44 were clinicians.

\section{Quality assessment}

Quality assessment tools from the NHLBI and CASP were used to assess the methodological quality of the included studies. The overall quality was rated as good, fair, or poor. Eight studies were rated as "fair" quality $[9,15,22,58,59,61,62,63]$. Whereas, two studies were rated as poor-quality assessment $[28,60]$. In general, most of the studies had small sample size. There were also issues in some studies related to lack of clarity in research questions and objectives; insufficient details provided about sampling, recruitment strategies and inclusion and exclusion criteria; lack of clarity in the rigor of the data analysis and reporting of ethics (see S2-S4 Tables).

\section{Telehealth technologies}

Regarding the assessed technologies, 7 papers using Real-Time method with 5 of these using Videoconferencing and with 2 of these using the Versatile and Integrated System for Telerehabilitation (VISYTER) system, and 3 papers used the Store-and-Forward method particularly the Naturalistic Observation Diagnostic Assessment (NODA) software.

\section{Outcomes}

The outcomes are structured and reported according to the telehealth methods (Real-Time/ Store-and-Forward) as each study had different results.

Real-time method. Videoconferencing. Videoconferencing software enables the families, medical teams and educational teams to meet and discuss care in real time from multiple locations. Three of the studies were by Reese et al. $[22,58,59]$ and this series of studies made a connection via Videoconferencing between the specialist hospital and the local health centre or school. Similarly, two studies $[60,61]$ used videoconferencing. The three studies by Reese et al. $[22,58,59]$ used simple videoconferencing to examine (1) the ability of the clinician to assess autism using videoconferencing, (2) the feasibility of clinicians to coach the patients using 
videoconferencing to correctly complete the assessment activities with their child and (3) the satisfaction of the families with the telemedicine assessment compared to standard face to face assessment. In terms of diagnostic accuracy, these studies found that the diagnosis assessment agreed with the evaluation team diagnosis; $82.5 \%(\mathrm{n}=28)$ of the time (for the face to face assessment) and $86 \%(n=24)$ (for the videoconference assessment) $[58,59]$. There was no significant difference in the ratings for the ADI-R and ADOS observations between those conducted face to face and those conducted online [58]. There was also excellent agreement between the clinicians across the diagnostic DSM-5 ASD criteria in person and when using the video conferencing approaches [22]. Therefore, these results indicated that there was no signif icant difference between the face to face and videoconferencing conditions. The final study by Reese et al. [59] found that access was improved and that there was an increase in the diagnostic services for families living in rural and underserved areas. There were 405 families who had the standard face to face assessment and 370 who had the telemedicine assessment. Only $16 \%$ returned the satisfaction survey. The children from the telemedicine method were from a wider area ( 74 counties compared to 35 in the face to face standard method), were diagnosed 3 years earlier and this method also identified older children who had not been previously diagnosed. The travel costs for those who used the telemedicine system would have been substantially more if they had needed to travel for each visit ( $\$ 100$ average cost if the video conferencing families had needed to travel versus $\$ 35$ average cost for face to face family). The parents were equally satisfied with the video conferencing and the face to face approaches. Two studies by Juarez et al. [62] and Stainbrook et al. [63] examined the accuracy of diagnosis using telehealth (videoconferencing) compared to the in-person assessment in 20 cases and feedback from both the families and clinicians on the acceptability of telemedicine [62]. Furthermore, the impact that telehealth had on the tertiary care referrals has been examined [63]. In terms of the accuracy of the diagnosis, using remote telemedicine procedures, the clinicians identified $75 \%$ of cases $(n=15 / 20)$ as having ASD. The clinicians rated themselves as "certain" or "very certain" about the classification for $75 \%$ of cases. Nineteen of 20 children were diagnosed with ASD by the in-person clinician. All of the children $(100 \%, n=15)$ who received the ASD diagnosis by telehealth were verified by a face to face assessment. However, $20 \%$ of cases $(n=4)$ diagnosed by the in-person assessment were not diagnosed remotely. In the evaluation of the feasibility and acceptability of the ASD diagnosis via telehealth ( $\mathrm{n}=45$ children), the clinicians reported that they were 'certain' or 'very certain' of their assessments in $86.67 \%$ of cases $(n=39)$. The clinicians were satisfied' or 'very satisfied' with the telemedicine in $80 \%$ of cases $(n=36)$ but they would rather to assess a child using an in-person assessment $24 \%$ of the time $(n=11)$, mainly for children who were then referred for a full evaluation $(n=6)$. This was because some psychosocial factors such as trauma history, complicated diagnostic profiles or technical issues with the telehealth platform ( $23.8 \%$ audio, $4.8 \%$ video quality concerns). However, $98 \%$ of caregivers were very satisfied with telemedicine experience. The telehealth assessment saved an average of $3.92 \mathrm{~h}$ of the anticipated families' travel time compared to going to the hospital-based ASD clinic.

The study examining the impact of telehealth (videoconferencing) on tertiary care referrals [63] found that the telehealth diagnostic consultation could positively impact referrals for the diagnostic assessment. Telehealth accelerated access to the diagnostic assessment compared to waiting at the tertiary centre. For example, in 2017, the system referred 323 children for evaluation which had a 15-month waiting time. In contrast, the families waited 11 weeks for a telemedicine visit and families kept more appointments. In addition, families were able to schedule and attend appointments. Most families (56 out of 63) preferred the remote method over travelling to the tertiary centre. The referrals to the centre for a complete evaluation were more aimed at the children with different presentations or it was down to family preference. 
Versatile and Integrated System for Tele-rehabilitation (VISYTER). Two studies [60, 61] used VISYTER, which incorporates videoconferencing, layout control, image and video presentations, an electronic scoring system and session recording/archiving. This was developed to support the diagnosis of adolescents and adults with possible ASD. It is an off the shelf hardware and it has its own secure server system. There is one desk at the clinician's site and the other at the local clinic (so the patient needs to attend the clinic in order to use the system). Two cameras are on the patient's side to capture their eye contact and hand movements. The clinician can zoom and control the camera and take snap shots.

These two studies $[60,61]$ examined the use of the VISYTER system for the assessment of adults. The participants were between the ages of 19 to $30 ; 26$ out of 46 met the inclusion/ exclusion criteria and of these, 14 used the remote method after the face to face method [61]. Of these, the majority had no preference and found both methods to be equal, 5 preferred face to face and 2 preferred remote. In this study, $75 \%$ of 14 were comfortable using the remote system, $78 \%$ felt the online system correctly represented their typical behaviours and interactions with others. The results supported using telehealth to conduct ADOS remotely. The majority of observations were comparable. However, there were differences in the "Empathy/Comments on others' Emotions", "Hand and Finger and other Complex Mannerisms" and "Asks for Information" which may be harder to assess remotely. In the team's second study [60], they found that the patients $(n=10)$ were satisfied with the remote system (average score of 6.5 out of 7) and that it scored high for learnability (6.14 out of 7), interface quality (6.5), interaction quality (6.3), that they were very comfortable with the system (6.5) and that they would use it again (6.17). However, the acceptability of telehealth as a substitute for face-to-face was lower (5.86 out of 7 )

Store-and-forward method. Naturalistic Observation Diagnostic Assessment (NODA). Three studies ( 2 by the same author (Nazneen) $[9,15,28]$ used the NODA system which includes NODA SmartCapture and NODA connect [28]. NODA SmartCapture enables the parents to upload short videos from their smart phones of their child's behaviour in specific situations (e.g. family meal, play time with others, playtime alone and parental concerns) and remotely share them with a clinician. The first three situations offered opportunities for the child to show play-based behaviours and social-communication skills. Each situation has instructions that contain recording instructions and a sample video to direct the parents about the environment setup. The medical professionals can send additional recording instructions to the parents and it is all uploaded to a webportal. The developmental history of the child is included in the webportal as well. NODA Connect has a list of tags that allows the clinician to determine the presence or absence of behaviours (e.g. no eye contact). These tags are automapped to the (DSM-5) diagnostic criteria. The assessment can be shared with other clinicians and the diagnostic report can be shared with the parents.

The two studies by Nazneen $[9,28]$ both evaluate the system for development. They involved interviewing the clinicians and families and an in-field evaluation in order to describe the experience of the clinicians and parents as they use NODA. The NODA development study [9] found that parents reported that it was easy to use with a score of 4 on a 5-point scale. The parents were able to follow the instructions and collected the right videos of the right length; $96 \%$ of videos recorded by the parents were reported as being clinically useful according to the clinicians. In total, 10 out of 11 of the assessments were given a diagnostic outcome that matched the child's previous diagnosis. The experience of the stakeholder's study [28] reported very positive findings for both the parents and clinicians which highlighted that the videos recorded at home allowed the clinicians to observe the evidence of naturalistic behaviours in children which is not possible in clinical settings and is thus otherwise inaccessible. Clinicians reported that the complete assessment of one child required one hour. They felt 
that the children with classical ASD symptoms would be easily and successfully diagnosed using NODA but children with different presentations would need an additional assessment It was suggested that NODA could be an effective early screener in these cases [28]. Cases where NODA would be most useful were identified as being with rural families and divorced or separated families; this is as both parents could upload and share videos separately. Therefore, the papers examining the usability of NODA were positive with 40 out of 44 parents feeling that they could use the system and that it would improve the time to diagnosis.

A study by Smith et al. [15] compared NODA (remote approach) with the gold standard (in person assessment) for 40 families with ASD children and 11 with non-ASD children. There was substantial agreement in the diagnosis, with a diagnostic agreement of $88.2 \%$ in the full sample and $85 \%$ in the subsample. The sensitivity was $84.5 \%$ and the specificity was between $94.4 \%$ (full sample) and $85.7 \%$ (subsample). The Kappa coefficients for inter-rater reliability showed $85 \%$ to $90 \%$ accuracy between the raters. Therefore, NODA was felt to be a reliable system that performed well compared to the gold standard. It was reported that NODA is not aimed at removing the need for upcoming assessments but that it is intended to accelerate the pathway to early interventions and treatment.

Collectively, these studies concluded that NODA has the potential to improve the efficiency of the ASD diagnostic process.

\section{Discussion}

The literature review found 10 papers ( 5 independent research teams) that used telehealth in the diagnosis of ASD. Eight of the studies were rated to be of "fair" quality, whereas two studies were rated "poor" using the NHLBI and CASP assessment tools. Most of the studies had small sample sizes (with the range of samples being 10 to 45) with descriptive analysis including a survey design and qualitative experience of the systems. Thus, future high-quality studies are needed to shed more light on this important area. There were 2 methods of using telehealth in the diagnosis of ASD; (1) Real-Time method e.g. Videoconferencing that enables a range of health professionals in different areas to meet in real time with the family to assess the child or adult, thus eliminating the issues of geographical spread. This means that access to specialists might be better facilitated. (2) A Store-and-Forward method as NODA system to upload videos of child's behaviours and interactions by parents/carers in order to enable the clinicians making an assessment remotely and sharing with parents/carers or other health professionals. This might allow the observations to be conducted by health professionals in the family home remotely, eliminating the need for the family to travel and also enabling for observations to be made in more naturalistic settings. Nearly all the systems identified are designed for ASD to enable the clinicians to undertake an assessment, e.g. the ability to score or mark videos, the ability to zoom in or out to observe facial expressions. This review of the literature found evidence that telehealth methods allow for collaboration and the sharing of experiences between the family, education, ASD experts and the university medical centres and could be equal to face to face methods in terms of satisfaction for the patient, family and clinician. They enable rural families to be seen at lower cost and reduce the time to diagnosis, particularly for those with more severe autism where there is in good agreement in terms of the diagnosis compared to the face to face methods. The findings from the review suggest that telehealth can potentially improve the efficiency of the diagnosis process for ASD.

All of the studies were from the US. However, implementing telehealth systems may facilitate the ASD assessment and diagnosis in other settings with different healthcare systems. In the UK, the multi-disciplinary team approach is one of the assessment methods used for ASD diagnosis, although it is not available in all areas $[3,64]$. Research has shown that it is difficult 
to get a multi-disciplinary team together, especially when they span different disciplines such as education and health [19]. Therefore, telehealth may provide support and help to a specialist ASD team, allowing them to meet remotely and provide a diagnosis regardless of their locations. Moreover, there are critical consequences of a delay in diagnosis as there has been reported a delay of approximately 3.5 years between seeking help and a confirmed diagnosis of ASD [7]. Telehealth technology may help to provide a timely diagnosis and, although it may not appropriate for complicated cases, it could be used as a screening tool for ASD. Consequently, this may increase the opportunity for early intervention as well as reducing the stress for individuals and their families.

\section{The strengths, limitations and future direction}

The strength of this review was in its comprehensive search strategy, intending to find all published studies regardless of method and research quality. However, there were some inconsistencies in the terminology across the studies and a general lack of precision in the descriptions of the telehealth technologies. Furthermore, this review only included papers that were published or written in English. This field is very new and only early design evaluations of the software and the clinician and family experiences of the system are available. There is a need for future high-quality studies using large sample sizes. More clarity in research questions, adequate details about sampling and inclusion and exclusion criteria, more clarity in the rigor of data analysis and reporting of ethics should be taken in the consideration. However, in this early stage, telehealth appears to offer the potential to improve the time to diagnosis of ASD. To date, the evidence suggests that the Real-Time and Store-and-Forward methods such as Videoconferencing and NODA system: (1) are acceptable to both the families and clinicians; (2) have good diagnostic accuracy; (3) enable families from a wider area to access professionals; (3) reduce costs for accessing care; (4) enable the natural behaviours in the home setting to be observed; and (5) may enable both parents in divorced families to contribute to the diagnostic process. This approach to diagnosis is complementary to other developments in this field such as the DAWBA (Development and Well-Being Assessment), which is a computerised assessment using the Strengths and Difficulties Questionnaire to reduce waiting times and inappropriate referrals [65]. The use of the existing telehealth methods could also greatly improve the validity of diagnosis in trials of interventions for ASD in terms of enabling the same team of health professions to see every patient in the trial regardless of location. Finally, the use of telehealth methods seems to be most helpful for those who have clear ASD characteristics as opposed to those whose characteristics may be borderline, and may be useful for the diagnosis of adults with ASD.

\section{The implications of the findings for practice}

This is the first step in examining the potential of using telehealth in conjunction with the current practices in order to improve the time to diagnosis for people with ASD. Larger studies with a randomised design will be needed to confirm that telehealth can improve the diagnosis times.

\section{Conclusion}

Early evidence about the use of telehealth for ASD diagnosis suggests that the systems available to date are acceptable to the families and clinicians. These systems have the potential to improve the time to diagnosis for the families and the people with ASD but warrant further study. 


\section{Supporting information}

S1 Table. (PRISMA-ScR) checklist.

(PDF)

S2 Table. Results of quality assessment tool (from CASP) for diagnostic studies.

(PDF)

S3 Table. Results of quality assessment tool (from the NHLBI) for observational cohort and cross-sectional studies.

S4 Table. Results of quality assessment tool (from CASP) for qualitative study. (PDF)

\section{Acknowledgments}

This research was supported by the National Centre for Population Health and Wellbeing Research.

\section{Author Contributions}

Conceptualization: Manahil Alfuraydan.

Investigation: Manahil Alfuraydan.

Methodology: Manahil Alfuraydan, Jodie Croxall, Sinead Brophy,

Supervision: Jodie Croxall, Sinead Brophy.

Writing - original draft: Manahil Alfuraydan.

Writing - review \& editing: Manahil Alfuraydan, Jodie Croxall, Lisa Hurt, Mike Kerr, Sinead Brophy.

\section{References}

1. American Psychiatric Association. Diagnostic and statistical manual of mental disorders (DSM-5). 5th ed. Arlington: American Psychiatric Association; 2013

2. Maenner MJ, Shaw KA, Baio J. Prevalence of autism spectrum disorder among children aged 8 years -autism and developmental disabilities monitoring network, 11 sites, United States, 2016. MMWR Surveill Summ. 2020; 69(4):1-12. https://doi.org/10.15585/mmwr.ss6904a1 PMID: 32214087

3. National Health Service. Getting diagnosed-Autism 2019. https://www.nhs.uk/conditions/autism/ getting-diagnosed/

4. The National Institute for Health and Care Excellence (NICE). Autism spectrum disorder in under 19s: recognition, referral and diagnosis 2019. https://www.nice.org.uk/guidance/cg128

5. Lord C, Rutter M, Le Couteur A. Autism Diagnostic Interview-Revised: a revised version of a diagnostic interview for caregivers of individuals with possible pervasive developmental disorders. J Autism Dev Disord. 1994; 24(5):659-85. https://doi.org/10.1007/bf02172145 PMID: 7814313

6. Lord C, Rutter M, DiLavore P, Risi S, Gotham K, Bishop S. Autism Diagnostic Observation Schedule, Second Edition (ADOS-2) Manual (Part II): Toddler Module. Torrance, CA: Western Psychological Services. 2012

7. Crane L, Chester JW, Goddard L, Henry LA, Hill E. Experiences of autism diagnosis: A survey of over 1000 parents in the United Kingdom. Autism. 2016; 20(2):153-62. https://doi.org/10.1177/ 1362361315573636 PMID: 25810370

8. Howlin $P$, Asgharian $A$. The diagnosis of autism and Asperger syndrome: findings from a survey of 770 families. Dev Med Child Neurol. 1999; 41(12):834-9 https://doi.org/10.1017/s0012162299001656 PMID: 10619282 
9. Nazneen N, Rozga A, Smith C, Oberleitner R, Abowd G, Arriaga R. A novel system for supporting autism diagnosis using home videos: iterative development and evaluation of system design. JMIR Mhealth Uhealth. 2015; 3(2). https://doi.org/10.2196/mhealth.4393 PMID: 26085230

10. Wiggins $L D$, Baio $\mathrm{J}$, Rice $C$. Examination of the time between first evaluation and first autism spectrum diagnosis in a population-based sample. J Dev Behav Pediatr. 2006; 27(2):S79-S87. https://doi.org/10. 1097/00004703-200604002-00005 PMID: 16685189

11. Matson JL. Clinical assessment and intervention for autism spectrum disorders. 1st ed. Amesterdam: Elsevier; 2011.

12. Siegel B. Getting the best for your child with autism: An expert's guide to treatment: New York: The Guilford Press; 2008 .

13. McEachin JJ, Smith T, Ivar Lovaas $O$. Long-term outcome for children with autism who received early intensive behavioral treatment. Am J Ment Retard. 1993; 97(4):359-72. PMID: 8427693

14. Rattazzi $A$. The importance of early detection and early intervention for children with autism spectrum conditions. Vertex. 2014; 25(116):290-4. PMID: 25546644

15. Smith CJ, Rozga A, Matthews N, Oberleitner R, Nazneen N, Abowd G. Investigating the accuracy of a novel telehealth diagnostic approach for autism spectrum disorder. Psychol Assess. 2017; 29(3):24552. http://doi.org/10.1037/pas0000317 PMID: 27196689

16. Zwaigenbaum L, Nicholas DB, Muskat B, Kilmer C, Newton AS, Craig WR, et al. Perspectives of Health Care Providers Regarding Emergency Department Care of Children and Youth with Autism Spectrum Disorder. J Autism Dev Disord. 2016; 46(5):1725-36. https://doi.org/10.1007/s10803-016-2703-y PMID: 26780909

17. Thomas KC, Ellis AR, McLaurin C, Daniels J, Morrissey JP. Access to care for autism-related services. J Autism Dev Disord. 2007; 37(10):1902-12. https://doi.org/10.1007/s10803-006-0323-7 PMID: 17372817

18. Oberleitner R, Elison-Bowers $P$, Reischl $U$, Ball J. Optimizing the personal health record with special video capture for the treatment of autism. J Dev Phys Disabil. 2007; 19(5):513-8.

19. Hurt L, Langley K, North $\mathrm{K}$, Southern $\mathrm{A}$, Copeland L, Gillard J, et al. Understanding and improving the care pathway for children with autism. Int J Health Care Qual Assur. 2019; 32(1):208-23. https://doi. org/10.1108/ijhcqa-08-2017-0153 PMID: 30859873

20. Vismara LA, McCormick C, Young GS, Nadhan A, Monlux K. Preliminary findings of a telehealth approach to parent training in autism. J Autism Dev Disord. 2013; 43(12):2953-69. https://doi.org/10. 1007/s10803-013-1841-8 PMID: 23677382

21. Maheu $M$, Whitten $P$, Allen A. E-Health, Telehealth, and Telemedicine: a guide to startup and success: San Francisco: Jossey-Bass; 2001

22. Reese R, Jamison T, Braun M, Wendland M, Black W, Hadorn M, et al. Brief Report: Use of Interactive Television in Identifying Autism in Young Children: Methodology and Preliminary Data. J Autism Dev Disord. 2015; 45(5):1474-82. https://doi.org/10.1007/s10803-014-2269-5 PMID: 25331323

23. Gros DF, Morland LA, Greene CJ, Acierno R, Strachan M, Egede LE, et al. Delivery of evidence-based psychotherapy via video telehealth. J Psychopathol Behav Assess. 2013; 35(4):506-21.

24. Dunn BE, Choi H, Almagro UA, Recla DL, Davis CW. Telepathology networking in VISN-12 of the Veterans Health Administration. Telemed J E Health. 2000; 6(3):349-54. https://doi.org/10.1089/ 153056200750040200 PMID: 11110638

25. Trott $P$, Blignault I. Cost evaluation of a telepsychiatry service in northern Queensland. J Telemed Telecare. 1998; 4(1_suppl):66-8. https://doi.org/10.1258/1357633981931515 PMID: 9640742

26. Wainer AL, Ingersoll BR. Increasing access to an ASD imitation intervention via a telehealth parent training program. J Autism Dev Disord. 2015; 45(12):3877-90. https://doi.org/10.1007/s10803-0142186-7 PMID: 25035089

27. Ingersoll B, Wainer A. Initial efficacy of Project ImPACT: A parent-mediated social communication intervention for young children with ASD. J Autism Dev Disord. 2013; 43(12):2943-52. https://doi.org/10. 1007/s10803-013-1840-9 PMID: 23689760

28. Nazneen N, Matthews N, Smith CJ, Rozga A, Abowd GD, Oberleitner R, et al. Use of a Novel Imaging Technology for Remote Autism Diagnosis: A Reflection on Experience of Stakeholders. Procedia Manuf. 2015; 3:293-300.

29. Alkhalifah $\mathrm{S}$, Aldhalaan $\mathrm{H}$. Telehealth services for children with autism spectrum disorders in rural areas of the Kingdom of Saudi Arabia: Overview and recommendations. JMIR Pediatr Parent. 2018; 1(2): e11402. https://doi.org/10.2196/11402 PMID: 31518306

30. Vismara LA, Young GS, Rogers SJ. Telehealth for expanding the reach of early autism training to parents. Autism Res Treat. 2012; 2012. https://doi.org/10.1155/2012/121878 PMID: 23227334 
31. lacono T, Dissanayake C, Trembath D, Hudry K, Erickson S, Spong J. Family and practitioner perspectives on telehealth for services to young children with autism. Stud Health Technol Inform. 2016 231:63-73. PMID: 27782017

32. Chedid RJ, Dew A, Veitch C. Barriers to the use of Information and Communication Technology by 2013; 60(3):197-205. https://doi.org/10.1111/1440-1630.12016 PMID: 23730785

33. Dunkley $\mathrm{C}$, Pattie L, Wilson L, McAllister L. A comparison of rural speech-language pathologists' and residents' access to and attitudes towards the use of technology for speech-language pathology servic delivery. Int J Speech Lang Pathol 2010; 12(4):333-43. https://doi.org/10.3109/1754950090345660 PMID: 20590518

34. Al-Safadi L. The Effects of Real-Time Interactive Multimedia Teleradiology System. Biomed Res Int 2016; 2016. https://doi.org/10.1155/2016/4126841 PMID: 27294118

35. Hsieh J-C, Li A-H, Yang C-C. Mobile, cloud, and big data computing: contributions, challenges, and new directions in telecardiology. Int J Environ Res Public Health. 2013; 10(11):6131-53. https://doi.org/ 10.3390/jerph10116131 PMID: 24232290

36. Hilty DM, Ferrer DC, Parish MB, Johnston B, Callahan EJ, Yellowlees PM. The effectiveness of telemental health: a 2013 review. Telemed J E Health. 2013; 19(6):444-54. https://doi.org/10.1089/tmj. 2013.0075 PMID: 23697504

37. Shores MM, Ryan-Dykes $P$, Williams RM, Mamerto B, Sadak T, Pascualy M, et al. Identifying undiagnosed dementia in residential care veterans: comparing telemedicine to in-person clinical examination. Int J Geriatr Psychiatry. 2004; 19(2):101-8. https://doi.org/10.1002/gps.1029 PMID: 14758575

38. McCrossan BA, Grant B, Morgan GJ, Sands AJ, Craig B, Casey FA. Diagnosis of congenital heart disease in neonates by videoconferencing: an eight-year experience. J Telemed Telecare. 2008; 14 (3):137-40. https://doi.org/10.1258/jtt.2008.003011 PMID: 18430281

39. Bowman R, Kennedy C, Kirwan J, Sze P, Murdoch I. Reliability of telemedicine for diagnosing and managing eye problems in accident and emergency departments. Eye (Lond). 2003; 17(6):743-6. https:/ doi.org/10.1038/sj.eye.6700489 PMID: 12928688

40. Aresti-Bartolome N, Garcia-Zapirain B. Technologies as support tools for persons with autistic spectrum disorder: a systematic review. Int J Environ Res Public Health. 2014; 11(8):7767-802. https://doi.org/ 10.3390/ijerph110807767 PMID: 25093654

41. Lindgren S, Wacker D, Suess A, Schieltz K, Pelzel K, Kopelman T, et al. Telehealth and autism: Treating challenging behavior at lower cost. Pediatrics. 2016; 137(Supplement 2): S167-S75. https://doi.org/ ing challenging behavior at lower cost. Pediat
10.1542/peds.2015-28510 PMID: 26908472

42. Machalicek W, Lequia J, Pinkelman S, Knowles C, Raulston T, Davis T, et al. Behavioral telehealth consultation with families of children with autism spectrum disorder. Behav Interv. 2016; 31(3):223-50.

43. Simacek J, Dimian AF, McComas JJ. Communication intervention for young children with severe neurodevelopmental disabilities via telehealth. J Autism Dev Disord. 2017; 47(3):744-67. http://doi.org/10. 1007/s10803-016-3006-z PMID: 28093677

44. Suess AN, Romani PW, Wacker DP, Dyson SM, Kuhle JL, Lee JF, et al. Evaluating the treatment fidelity of parents who conduct in-home functional communication training with coaching via telehealth. $J$ Behav Educ. 2014; 23(1):34-59.

45. Suess AN, Wacker DP, Schwartz JE, Lustig N, Detrick J. Preliminary evidence on the use of telehealth in an outpatient behavior clinic. J Appl Behav Anal. 2016; 49(3):686-92. http://doi.org/10.1002/jaba.305 PMID: 27001117

46. Wacker DP, Lee JF, Dalmau YCP, Kopelman TG, Lindgren SD, Kuhle J, et al. Conducting functional communication training via telehealth to reduce the problem behavior of young children with autism. $J$ Dev Phys Disabil. 2013; 25(1):35-48. https://doi.org/10.1007/s10882-012-9314-0 PMID: 23543855

47. Wacker DP, Lee JF, Dalmau YCP, Kopelman TG, Lindgren SD, Kuhle J, et al. Conducting functional analyses of problem behavior via telehealth. J Appl Behav Anal. 2013; 46(1):31-46. https://doi.org/10. 1002/jaba.29 PMID: 24114083

48. Ingersoll B, Berger NI. Parent Engagement With a Telehealth-Based Parent-Mediated Intervention Program for Children With Autism Spectrum Disorders: Predictors of Program Use and Parent Outcomes. J Med Internet Res. 2015; 17(10):e227. https://doi.org/10.2196/jmir.4913 PMID: 26443557

49. Pickard KE, Wainer AL, Bailey KM, Ingersoll BR. A mixed-method evaluation of the feasibility and acceptability of a telehealth-based parent-mediated intervention for children with autism spectrum disorder. Autism. 2016; 20(7):845-55. https://doi.org/10.1177/1362361315614496 PMID: 26862084

50. Boisvert M, Lang R, Andrianopoulos M, Boscardin ML. Telepractice in the assessment and treatment of individuals with autism spectrum disorders: A systematic review. Developmental neurorehabilitation. 2010; 13(6):423-32. https://doi.org/10.3109/17518423.2010.499889 PMID: 20887200 
51. Boisvert $\mathrm{M}, \mathrm{Hall} \mathrm{N}$. The use of telehealth in early autism training for parents: a scoping review. Smart Homecare Technology and Telehealth. 2014; 2:19-27.

52. Meadan $\mathrm{H}$, Daczewitz ME. Internet-based intervention training for parents of young children with disabilities: a promising service-delivery model. Early child development and care. 2015; 185(1):155-69.

53. Munn Z, Peters MD, Stern C, Tufanaru C, McArthur A, Aromataris E. Systematic review or scoping review? Guidance for authors when choosing between a systematic or scoping review approach. BMC Med Res Methodol. 2018; 18(1):143. https://doi.org/10.1186/s12874-018-0611-x PMID: 30453902

54. Arksey H, O'Malley L. Scoping studies: towards a methodological framework. Int J Soc Res Methodol. $2005 ; 8(1): 19-32$

55. Tricco AC, Lillie E, Zarin W, O'Brien KK, Colquhoun H, Levac D, et al. PRISMA extension for scoping reviews (PRISMA-ScR): checklist and explanation. Ann Intern Med. 2018; 169(7):467-73. https://doi. org/10.7326/M18-0850 PMID: 30178033

56. National Heart, Lung, and Blood Institute. Study Quality Assessment Tools. https://www.nhlbi.nih.gov/ health-pro/\%20guidelines/in-develop/cardiovascular-\%20risk-reduction/tools.

57. Critical Appraisal Skills Programme (CASP). CASP Checklists. https://casp-uk.net/casp-toolschecklists/.

58. Reese RM, Jamison R, Wendland M, Fleming K, Braun MJ, Schuttler JO, et al. Evaluating interactive videoconferencing for assessing symptoms of autism. Telemed J E Health. 2013; 19(9):671-7. https:// doi.org/10.1089/tmj.2012.0312 PMID: 23870046

59. Reese RM, Braun MJ, Hoffmeier S, Stickle L, Rinner L, Smith C, et al. Preliminary Evidence for the Integrated Systems Using Telemedicine. Telemed J E Health. 2015; 21(7):581-7. https://doi.org/10.1089/ grated Systems Using Telemedicine

60. Parmanto B, Pulantara IW, Schutte JL, Saptono A, McCue MP. An integrated telehealth system for remote administration of an adult autism assessment. Telemed J E Health. 2013; 19(2):88-94. https:// doi.org/10.1089/tmj.2012.0104 PMID: 23230821

61. Schutte JL, McCue MP, Parmanto B, McGonigle J, Handen B, Lewis A, et al. Usability and reliability of a remotely administered adult autism assessment, the autism diagnostic observation schedule (ADOS) a remotely administered adult autism assessment, the autism diagnostic observation schedule (ADO 25569603

62. Juarez AP, Weitlauf AS, Nicholson A, Pasternak A, Broderick N, Hine J, et al. Early Identification of ASD Through Telemedicine: Potential Value for Underserved Populations. J Autism Dev Disord. 2018; 48(8):2601-10. https://doi.org/10.1007/s10803-018-3524-y PMID: 29527626

63. Stainbrook JA, Weitlauf AS, Juarez AP, Taylor JL, Hine J, Broderick N, et al. Measuring the service system impact of a novel telediagnostic service program for young children with autism spectrum disorder. tem impact of a novel telediagnostic service program for young children with autism spectrum dicer

64. National Autistic Society. All about diagnosis 2018. https://www. autism.org.uk/about/diagnosis.aspx

65. Goodman R, Ford T, Richards $\mathrm{H}$, Gatward R, Meltzer $\mathrm{H}$. The development and well-being assessment: Description and initial validation of an integrated assessment of child and adolescent psychopathology. J Child Psychol Psychiatry. 2000; 41(5):645-55. PMID: 10946756 


\section{Appendix 2: (PRISMA-ScR) Checklist}

\begin{tabular}{|c|c|c|c|}
\hline SECTION & ITEM & PRISMA-ScR CHECKLIST ITEM & $\begin{array}{l}\text { REPORTED } \\
\text { ON PAGE \# }\end{array}$ \\
\hline \multicolumn{4}{|l|}{ TITLE } \\
\hline Title & 1 & Identify the report as a scoping review. & Title, page 49 \\
\hline \multicolumn{4}{|l|}{ ABSTRACT } \\
\hline $\begin{array}{l}\text { Structured } \\
\text { summary }\end{array}$ & 2 & $\begin{array}{l}\text { Provide a structured summary that includes (as } \\
\text { applicable): background, objectives, eligibility } \\
\text { criteria, sources of evidence, charting methods, } \\
\text { results, and conclusions that relate to the review } \\
\text { questions and objectives. }\end{array}$ & $\begin{array}{l}\text { Abstract, page } \\
238\end{array}$ \\
\hline \multicolumn{4}{|l|}{ INTRODUCTION } \\
\hline Rationale & 3 & $\begin{array}{l}\text { Describe the rationale for the review in the context } \\
\text { of what is already known. Explain why the review } \\
\text { questions/objectives lend themselves to a scoping } \\
\text { review approach. }\end{array}$ & $\begin{array}{l}\text { Introduction, } \\
\text { page } 49-53\end{array}$ \\
\hline Objectives & 4 & $\begin{array}{l}\text { Provide an explicit statement of the questions and } \\
\text { objectives being addressed with reference to their } \\
\text { key elements (e.g., population or participants, } \\
\text { concepts, and context) or other relevant key } \\
\text { elements used to conceptualize the review } \\
\text { questions and/or objectives. }\end{array}$ & $\begin{array}{l}\text { Introduction, } \\
\text { page } 53\end{array}$ \\
\hline \multicolumn{4}{|l|}{ METHODS } \\
\hline $\begin{array}{l}\text { Protocol and } \\
\text { registration }\end{array}$ & 5 & $\begin{array}{l}\text { Indicate whether a review protocol exists; state if } \\
\text { and where it can be accessed (e.g., a Web } \\
\text { address); and if available, provide registration } \\
\text { information, including the registration number. }\end{array}$ & $\mathrm{N} / \mathrm{A}$ \\
\hline Eligibility criteria & 6 & $\begin{array}{l}\text { Specify characteristics of the sources of evidence } \\
\text { used as eligibility criteria (e.g., years considered, } \\
\text { language, and publication status), and provide a } \\
\text { rationale. }\end{array}$ & $\begin{array}{l}\text { Method, page } \\
55-56\end{array}$ \\
\hline $\begin{array}{l}\text { Information } \\
\text { sources* }\end{array}$ & 7 & $\begin{array}{l}\text { Describe all information sources in the search } \\
\text { (e.g., databases with dates of coverage and contact } \\
\text { with authors to identify additional sources), as } \\
\text { well as the date the most recent search was } \\
\text { executed. }\end{array}$ & $\begin{array}{l}\text { Method, page } \\
54-56\end{array}$ \\
\hline Search & 8 & $\begin{array}{l}\text { Present the full electronic search strategy for at } \\
\text { least } 1 \text { database, including any limits used, such } \\
\text { that it could be repeated. }\end{array}$ & $\begin{array}{l}\text { Methods; Box } \\
1 \text {, page } 55\end{array}$ \\
\hline $\begin{array}{l}\text { Selection of } \\
\text { sources of } \\
\text { evidence } \uparrow\end{array}$ & 9 & $\begin{array}{l}\text { State the process for selecting sources of evidence } \\
\text { (i.e., screening and eligibility) included in the } \\
\text { scoping review. }\end{array}$ & $\begin{array}{l}\text { Method, page } \\
54-56\end{array}$ \\
\hline $\begin{array}{l}\text { Data charting } \\
\text { process }\end{array}$ & 10 & $\begin{array}{l}\text { Describe the methods of charting data from the } \\
\text { included sources of evidence (e.g., calibrated } \\
\text { forms or forms that have been tested by the team } \\
\text { before their use, and whether data charting was } \\
\text { done independently or in duplicate) and any } \\
\text { processes for obtaining and confirming data from } \\
\text { investigators. }\end{array}$ & $\begin{array}{l}\text { Method, page } \\
54-56\end{array}$ \\
\hline Data items & 11 & $\begin{array}{l}\text { List and define all variables for which data were } \\
\text { sought and any assumptions and simplifications } \\
\text { made. }\end{array}$ & N/A \\
\hline $\begin{array}{l}\text { Critical appraisal } \\
\text { of individual } \\
\text { sources of } \\
\text { evidence }\end{array}$ & 12 & $\begin{array}{l}\text { If done, provide a rationale for conducting a } \\
\text { critical appraisal of included sources of evidence; } \\
\text { describe the methods used and how this } \\
\text { information was used in any data synthesis (if } \\
\text { appropriate). }\end{array}$ & $\begin{array}{l}\text { Method, page } \\
56\end{array}$ \\
\hline Synthesis of & 13 & Describe the methods of handling and & Method, page \\
\hline
\end{tabular}




\begin{tabular}{|c|c|c|c|}
\hline results & & summarizing the data that were charted. & 56 \\
\hline \multicolumn{4}{|l|}{ RESULTS } \\
\hline $\begin{array}{l}\text { Selection of } \\
\text { sources of } \\
\text { evidence }\end{array}$ & 14 & $\begin{array}{l}\text { Give numbers of sources of evidence screened, } \\
\text { assessed for eligibility, and included in the review, } \\
\text { with reasons for exclusions at each stage, ideally } \\
\text { using a flow diagram. }\end{array}$ & \begin{tabular}{|l} 
Results, page \\
59; Figure 3-1
\end{tabular} \\
\hline $\begin{array}{l}\text { Characteristics of } \\
\text { sources of } \\
\text { evidence }\end{array}$ & 15 & $\begin{array}{l}\text { For each source of evidence, present } \\
\text { characteristics for which data were charted and } \\
\text { provide the citations. }\end{array}$ & \begin{tabular}{|l|} 
Results, page \\
$58 ;$ Table 3-1, \\
page 60-64 \\
\end{tabular} \\
\hline $\begin{array}{l}\text { Critical appraisal } \\
\text { within sources of } \\
\text { evidence }\end{array}$ & 16 & $\begin{array}{l}\text { If done, present data on critical appraisal of } \\
\text { included sources of evidence (see item 12). }\end{array}$ & $\begin{array}{l}\text { Results, page } \\
65 ; \text { Table3-2, } \\
\text { Table 3-3, } \\
\text { Table 3-4, } \\
\text { page } 66-69\end{array}$ \\
\hline $\begin{array}{l}\text { Results of } \\
\text { individual sources } \\
\text { of evidence }\end{array}$ & 17 & $\begin{array}{l}\text { For each included source of evidence, present the } \\
\text { relevant data that were charted that relate to the } \\
\text { review questions and objectives. }\end{array}$ & $\begin{array}{l}\text { Results, page } \\
\text { 70-75; Table } \\
\text { 3-1, page } 60- \\
64\end{array}$ \\
\hline $\begin{array}{l}\text { Synthesis of } \\
\text { results }\end{array}$ & 18 & $\begin{array}{l}\text { Summarize and/or present the charting results as } \\
\text { they relate to the review questions and objectives. }\end{array}$ & $\begin{array}{l}\text { Results, page } \\
70-75\end{array}$ \\
\hline \multicolumn{4}{|l|}{ DISCUSSION } \\
\hline $\begin{array}{l}\text { Summary of } \\
\text { evidence }\end{array}$ & 19 & $\begin{array}{l}\text { Summarize the main results (including an } \\
\text { overview of concepts, themes, and types of } \\
\text { evidence available), link to the review questions } \\
\text { and objectives, and consider the relevance to key } \\
\text { groups. }\end{array}$ & $\begin{array}{l}\text { Discussion, } \\
\text { page 76-78 }\end{array}$ \\
\hline Limitations & 20 & $\begin{array}{l}\text { Discuss the limitations of the scoping review } \\
\text { process. }\end{array}$ & $\begin{array}{l}\text { Discussion, } \\
\text { page } 77\end{array}$ \\
\hline Conclusions & 21 & $\begin{array}{l}\text { Provide a general interpretation of the results with } \\
\text { respect to the review questions and objectives, as } \\
\text { well as potential implications and/or next steps. }\end{array}$ & $\begin{array}{l}\text { Conclusion, } \\
\text { page } 78\end{array}$ \\
\hline \multicolumn{4}{|l|}{ FUNDING } \\
\hline Funding & 22 & $\begin{array}{l}\text { Describe sources of funding for the included } \\
\text { sources of evidence, as well as sources of funding } \\
\text { for the scoping review. Describe the role of the } \\
\text { funders of the scoping review. }\end{array}$ & - \\
\hline
\end{tabular}




\section{Swansea University Medical School Ysgol Feddygaeth Prifysgol Abertawe}

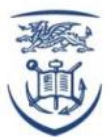

Swansea University Prifysgol Abertawe

\section{Are you Parent or Carer of a Child with Autism Spectrum Disorder (ASD) Living in Wales?}

\section{We Would Like to Reduce the Time to Get the Diagnosis! We Need Your Help!}

This will involve completing an online questionnaire. The answers you give could help to improve the diagnostic services in Wales. All information will be anonymous and kept confidential.

All completed questionnaires will be entered into a prize draw to win a $£ 25$ Amazon voucher (8 participants).

Who can participate?
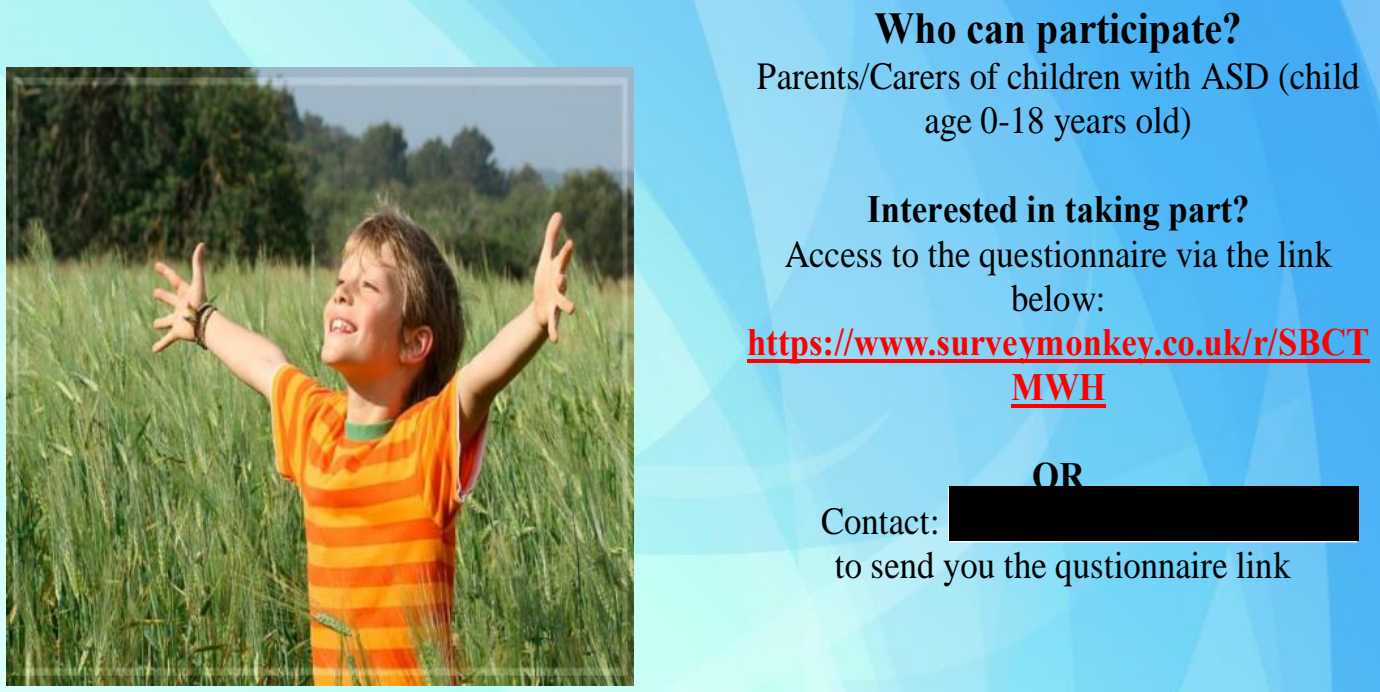

Thank you very much for your time

Manahil Alfuraydan is a PhD student at the Swansea University Medical School, supervised by Dr Jodie Croxall and Prof. Sinead Brophy. The study has been approved by Research Ethics Committee in Swansea University, to protect your safety, rights, wellbeing, and dignity. 


\section{Appendix 4: Participant Information Sheet}

Exploring Parents'/Carers' Experience in Obtaining a Diagnosis of ASD for their Children and their Acceptance of Using an Online Diagnostics System (NODA)

Participant Information Sheet

Invitation to participate in the study You are invited to consider participating in a study that is being conducted as part of a Doctoral Degree in the Swansea University Medical School. Before deciding to participate in this study, we recommend that you take time to read the following information which will provide you with details about the project and what your involvement would entail if you decide to take part in the study.

What is the purpose?

Part (1): aims to give the parents/carers an opportunity to explore their thoughts on their experience with their children's Autism Spectrum Disorder (ASD) diagnosis in Wales.

Part (2): aims to find out how parents/carers of children with ASD would respond to using NODA system for remote ASD diagnosis.

Do I have to take part? No, taking part in the study is voluntary and you can withdraw at any time, without having to give a reason.

What will happen to me if I take part? If you agree to participate in the study, make sure you read this sheet and provide consent to participate in the study. The questionnaire will take about 20 minutes to complete.

What will happen if I do not want to carry on with the study?There are no implications for you if you withdraw. It is your right to withdraw from the study at any time.

What are the possible benefits of taking part? The answers you provide could help to understand the situation and find the ways in which diagnostic services to parents can be better delivered and accessed.

What happens when the study finishes? Access to the questionnaire will be removed and the data will be analysed. If you are interested in the results of the questionnaire, please contact ata will be analysed. if you

Will my taking part in the study be kept confidential?Yes. The information collected from you during the course of the research study will be anonymous, ensuring your privacy and confidentiality at all time.

Who is organising and funding the research? This research is organised by the Swansea University Medical School as part of a PhD research and the student is funded by Saudi Arabian Cultural Bureau in London. The study has been reviewed by Research Ethics Committee in Swansea University, to protect your safety, rights, wellbeing, and dignity.

Further information and contact details: if you have any questions regarding this study, or would like more information, please contact: Manahil Alfuraydan, Researcher or Dr Jodie Croxall, PhD research supervisor

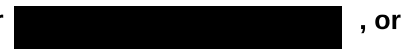


Prof. Sinead Brophy, PhD research supervisor ( 


\section{Appendix 5: Consent Form}

Exploring Parents'/Carers' Experience in Obtaining a Diagnosis of ASD for their Children and their Acceptance of Using an Online Diagnostics System (NODA)

\section{Consent Form}

I can confirm that I have read the participant information sheet for the online questionnaire. I have had time to consider, ask any questions that I may have regarding the contents of the questionnaire and I am satisfied with the answers given.

I understand that my participation is voluntary and I am free to withdraw from participating from this study without being penalised or disadvantaged in any way. This will not affect my legal rights, dignity or freedom in doing so.

I understand the information that I give as part of this questionnaire will be viewed by researcher and her supervisors; I give permission for them to access my data for this research.

* 1. Do you consent to participate in this study?

Yes

No 


\section{Appendix 6: Debriefing Form}

Exploring Parents'/Carers' Experience in Obtaining a Diagnosis of ASD for their Children and their Acceptance of Using an Online Diagnostics System (NODA)

\section{Debriefing Form}

Thank you for your participation in this study. The data will be used for research purposes only and all information produced by this study will be anonymous. All participants will be treated confidentially, and all information will be stored securely, thus, protecting privacy, confidentiality and anonymity.

If you would like to receive a copy of the results at the end of the study, please contact: 842703@swansea.ac.uk

If your participation in this study has caused you some concerns, anxiety, or distress, you may contact NAS Helpline (08088004104).

If you have any further questions about the studv. please contact:

Manahil Alfuraydan, Researcher (4), or

Dr Jodie Croxall, PhD research supervisor (

Prof. Sinead Brophy, PhD research supervisor

THANK YOU AGAIN FOR YOUR PARTICIPATION. 


\section{Appendix 7: Online Questionnaire}

Exploring Parents'/Carers' Experience in Obtaining a Diagnosis of ASD for their Children and their Acceptance of Using an Online Diagnostics System (NODA)

Welcome to My Survey

Thank you for participating in our survey. Your feedback is important.

We would like to ask you to share:

Part (1): Your experience in obtaining a diagnosis of ASD for your child.

Part (2): Your thoughts on using an online ASD diagnostic system.

The questionnaire will take around 20 minutes to complete.

All completed questionnaires will be entered into a prize draw to win a £25 Amazon voucher (8 participants). If you wish to be entered into the draw, please enter your email at the end of the survey.

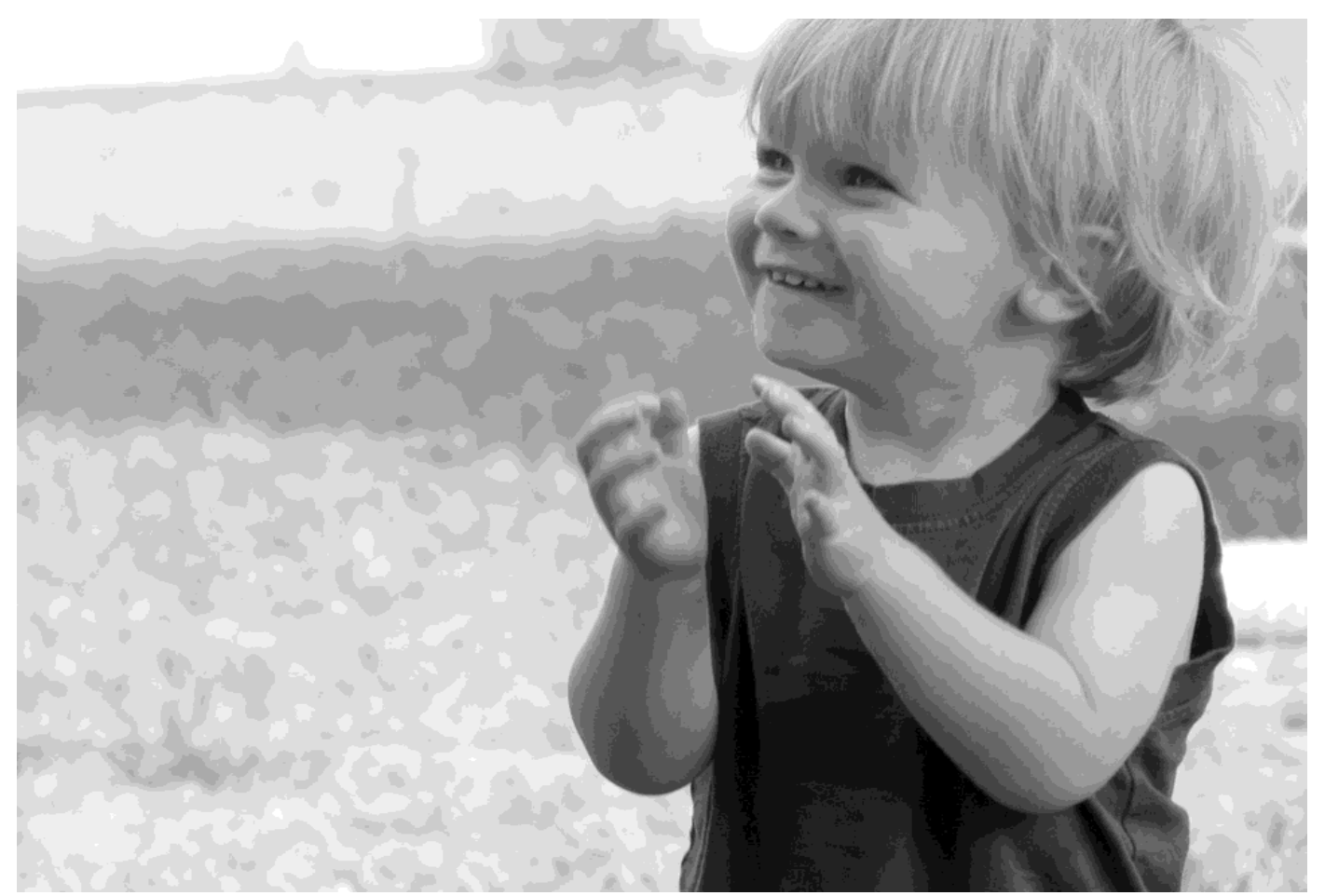


Exploring Parents'/Carers' Experience in Obtaining a Diagnosis of ASD for their Children and their Acceptance of Using an Online Diagnostics System (NODA)

\section{Participant Information Sheet}

Invitation to participate in the study You are invited to consider participating in a study that is being conducted as part of a Doctoral Degree in the Swansea University Medical School. Before deciding to participate in this study, we recommend that you take time to read the following information which will provide you with details about the project and what your involvement would entail if you decide to take part in the study.

What is the purpose?

Part (1): aims to give the parents/carers an opportunity to explore their thoughts on their experience with their children's Autism Spectrum Disorder (ASD) diagnosis in Wales.

Part (2): aims to find out how parents/carers of children with ASD would respond to using NODA system for remote ASD diagnosis.

Do I have to take part? No, taking part in the study is voluntary and you can withdraw at any time, without having to give a reason.

What will happen to me if I take part? If you agree to participate in the study, make sure you read this sheet and provide consent to participate in the study. The questionnaire will take about 20 minutes to complete.

What will happen if I do not want to carry on with the study?There are no implications for you if you withdraw. It is your right to withdraw from the study at any time.

What are the possible benefits of taking part? The answers you provide could help to understand the situation and find the ways in which diagnostic services to parents can be better delivered and accessed.

What happens when the study finishes? Access to the questionnaire will be removed and the data will be analysed. If you are interested in the results of the questionnaire, please contact

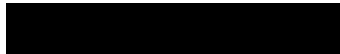

Will my taking part in the study be kept confidential?Yes. The information collected from you during the course of the research study will be anonymous, ensuring your privacy and confidentiality at all time.

Who is organising and funding the research? This research is organised by the Swansea University Medical School as part of a PhD research and the student is funded by Saudi Arabian Cultural Bureau in London. The study has been reviewed by Research Ethics Committee in Swansea University, to protect your safety, rights, wellbeing, and dignity.

Further information and contact details: if you have any questions regarding this study, or would like more information, please contact:

Manahil Alfuraydan, Researcher or

Dr Jodie Croxall, PhD research supervisor or or 
Prof. Sinead Brophy, PhD research supervisor ( 


\section{Consent Form}

I can confirm that I have read the participant information sheet for the online questionnaire. I have had time to consider, ask any questions that I may have regarding the contents of the questionnaire and I am satisfied with the answers given.

I understand that my participation is voluntary and I am free to withdraw from participating from this study without being penalised or disadvantaged in any way. This will not affect my legal rights, dignity or freedom in doing so.

I understand the information that I give as part of this questionnaire will be viewed by researcher and her supervisors; I give permission for them to access my data for this research.

* 1. Do you consent to participate in this study?

Yes

No 
Exploring Parents'/Carers' Experience in Obtaining a Diagnosis of ASD for their Children and their Acceptance of Using an Online Diagnostics System (NODA)

\section{PART (1) Experience of your Child's ASD Diagnosis}

\section{Section 1: Demographic of the respondent (you)}

*2. What is your gender?

Male

Female

* 3 . What is your age?

* 4 . What is your current marital status?
Single with partner
Sperated
Single without partner
Divorced
Married
Widowed
Other (please specify)

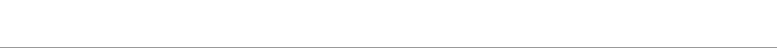

${ }^{*} 5$. What is the highest level of education completed?

No qualification

Bachelor Degree

Left School at 16 with qualification

Master Degree

Left School at 18 with qualification

Doctorate Degree

Other (please specify)

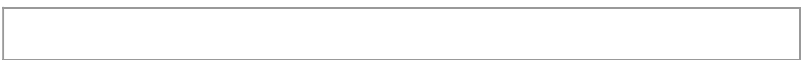

*6. Which area in Wales do you live?

South Wales

Dyfed-Powys

Gwent

North Wales

* 7. Please provide the first part of your post code? 
Exploring Parents'/Carers' Experience in Obtaining a Diagnosis of ASD for their Children and their Acceptance of Using an Online Diagnostics System (NODA)

* 8 . What is your relationship to the child?

\begin{tabular}{ll} 
Mother & $\bigcirc$ Grandparent \\
Father & $\bigcirc$ Legal Guardian \\
Other (please specify) & \\
\hline
\end{tabular}


Exploring Parents'/Carers' Experience in Obtaining a Diagnosis of ASD for their Children and their Acceptance of Using an Online Diagnostics System (NODA)

* 9 . How many children do you have?
1
4
2
5
3
$6+$

* 10. How many children currently living in your family household have autism- ASD?
1 1
3
- 2
4+ 
Exploring Parents'/Carers' Experience in Obtaining a Diagnosis of ASD for their Children and their Acceptance of Using an Online Diagnostics System (NODA)

\section{Section 2: General Information about the child with Autism Spectrum Disorder (ASD)}

* 11 . What is the child's age? (if there is more than one child with ASD, provide the age of the youngest)

* 12. What is your child's gender?

Male

Female

* 13 . What is the verbal ability of your child?

Does not talk

Uses single words only (e.g. "Bubbles")

Uses two- or three-word phrases (e.g. "Daddy shoe")

Uses sentences with four or more words (e.g. "I want more juice")

Uses complex sentences (e.g. "I am tired and I want to sleep")

* 14. How old was your child when you began to have concerns about his/her development?(Please enter the age in years e.g. under 1 year, 2 years, 3 years.....etc).

* 15. There are many reasons why a parent/carer might be concerned about a child's development. Below is a list of behaviors that can cause a parent/carer to be concerned. Think about the first concerns you had and tick all that apply below.
Had medical problems such as seizures, lack of physical growth, or stomach problems
Some speech skills that he/she had already developed were lost
Didn't make eye contact when talking or playing with others
Didn't seem to understand what you or other adults said to him/her
Didn't respond when called or didn't respond to sound
Didn't seem to understand nonverbal communication, such as understanding what you meant by the tone of voice you used or your facial expressions or other body language cues
Had problems with fine motor skills such as using scissors or drawing with crayons
Had difficulty playing or interacting with others, or played alone "in her/her own world"
Had behavioral difficulties such as sleeping or eating problems, high activity level, wandering, tantrums, aggressive or destructive behavior
Insisted on sameness or had difficulties with change
Had difficulty learning new skills such as toilet training or getting dressed
Had problems with coordination or gross motor skills such walking
Had difficulty learning new things such as the alphabet or numbers
Talked later than usual for most children
Was not talking at all
Had unusual gestures or movements such as hand-
flapping, toe-walking, or self- spinning
Did not talk as well as other children that were the same
age 
Exploring Parents'/Carers' Experience in Obtaining a Diagnosis of ASD for their Children and their Acceptance of Using an Online Diagnostics System (NODA)

\section{Section 3: The diagnostic process}

* 16. How old was your child when you first sought help?(Please enter the age in years e.g. under 1 year, 2 years, 3 years.....etc).

* 17 . Who did you see at this time?

GP (family doctor)

Neurologist

Health Visitor

Teacher

Paediatrician

Nurse

Child Psychiatrist

Social worker

Psychologist

Unsure or don't know

Other (please specify)

*18. What happened then?

Diagnosis made

Referred to other professional

Sent for tests

Told "no problem" or told not to worry (e.g., "they'll grow out of it")

Told to return if problems did not improve

Other (please specify)

* 19. How many professionals did you discuss your concerns with before receiving an ASD diagnosis for your child?
1
4
$\bigcirc 2$
5 and more
3
Unsure or don't know 


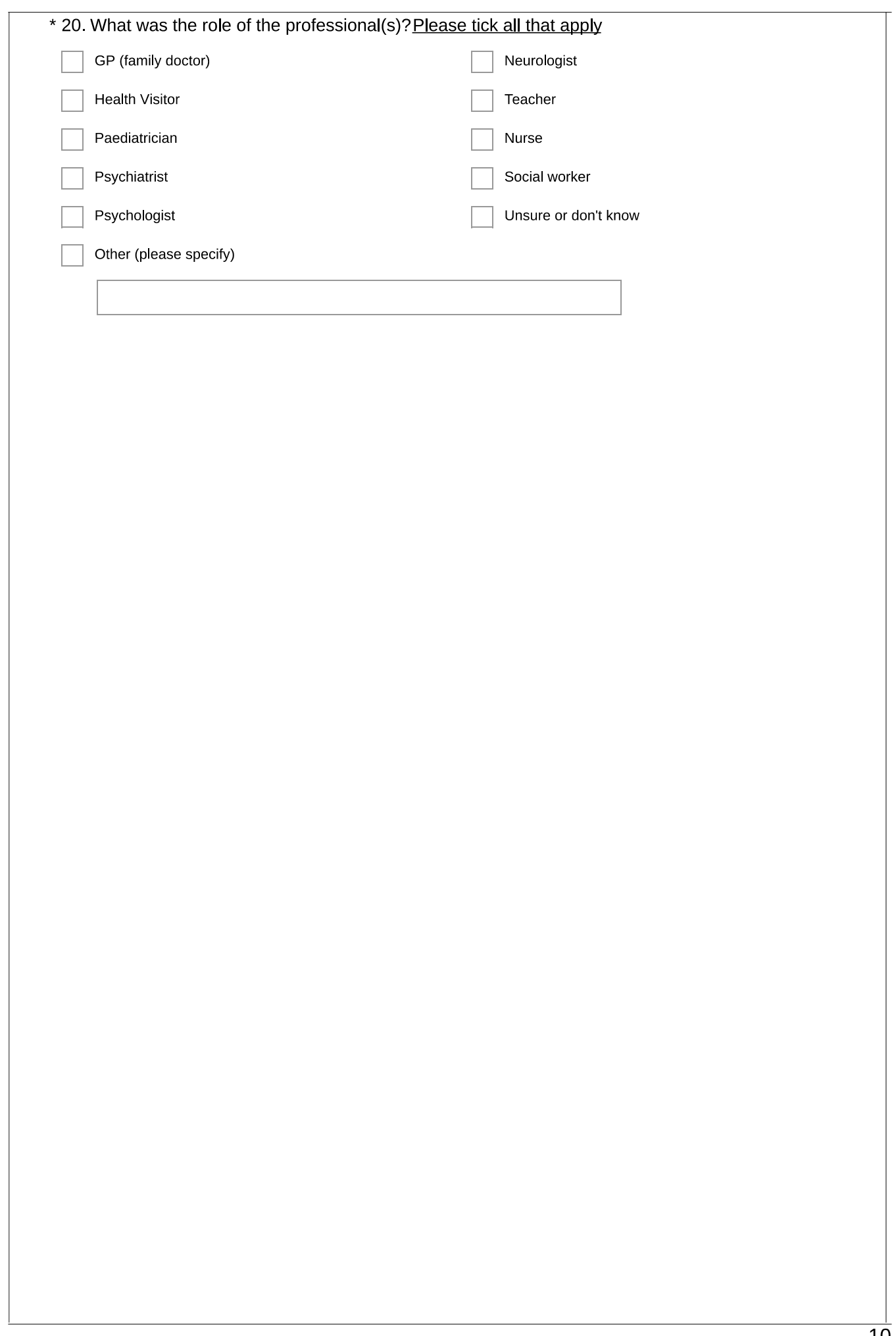


Exploring Parents'/Carers' Experience in Obtaining a Diagnosis of ASD for their Children and their Acceptance of Using an Online Diagnostics System (NODA)

\section{Section 4: Final Diagnosis and Satisfaction}

* 21 . What is your child's current diagnosis?

Autism

Asperger syndrome

Autism Spectrum Disorder

Pervasive Developmental Disorder:Not Otherwise Specified

Childhood Disintegrative Disorder

Rett syndrome

Other (please specify)

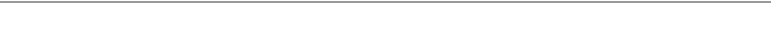

* 22. What was the role of the person who diagnosed your child? Please tick all that apply.

Paediatrician

Speech and language therapist

Speech therapist

Occupational therapist

Clinical psychologist

Other (please specify)

\section{Educational psychologist}

Psychiatrist

Neurologist

Unsure or don't know

* 23. How old was the child at the time of diagnosis of ASD?(Please enter the age in years e.g. under 1 year, 2 years, 3 years.....etc).

* 24. How long (months/years) did it take between first seeking help and receiving a diagnosis of ASD? (Please enter the time in months or years e.g 10 months, 2 years....etc)

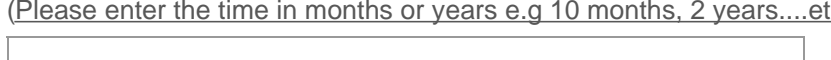

* 25. Did you receive a written report on the diagnosis?

Yes

Not sure

No 
* 26. Was there a follow-up appointment with the same professional following the diagnosis?

Yes

Not sure

No

* 27. Were you offered practical help/support by health professionals during the process of seeking a diagnosis?

Yes, offered directly (i.e., if assistance was provided by, for $\bigcirc$ No

example, arranging appointments for you)

Yes, signposted towards (i.e., if information was provided but it was left to you to arrange contact)

Other (please specify)

* 28. Were you offered practical help/support by health professionals in follow-up appointments after diagnosis?

Yes, offered directly (i.e., if assistance was provided by, for $\bigcirc$ No example, arranging appointments for you)

Yes, signposted towards (i.e., if information was provided but it was left to you to arrange contact)

Other (please specify)

* 29. How satisfied were you with the Time taken to get diagnosis?

Very dissatisfied

Quite satisfied

Quite dissatisfied

Very satisfied

Neither satisfied nor dissatisfied

* 30 . How satisfied were you with the Information given at diagnosis?

Very dissatisfied

Quite satisfied

Quite dissatisfied

Very satisfied

Neither satisfied nor dissatisfied

* 31. Overall, how satisfied were you with the Diagnostic Process AS A WHOLE?

Very dissatisfied

Quite satisfied

Quite dissatisfied

Very satisfied

Neither satisfied nor dissatisfied 


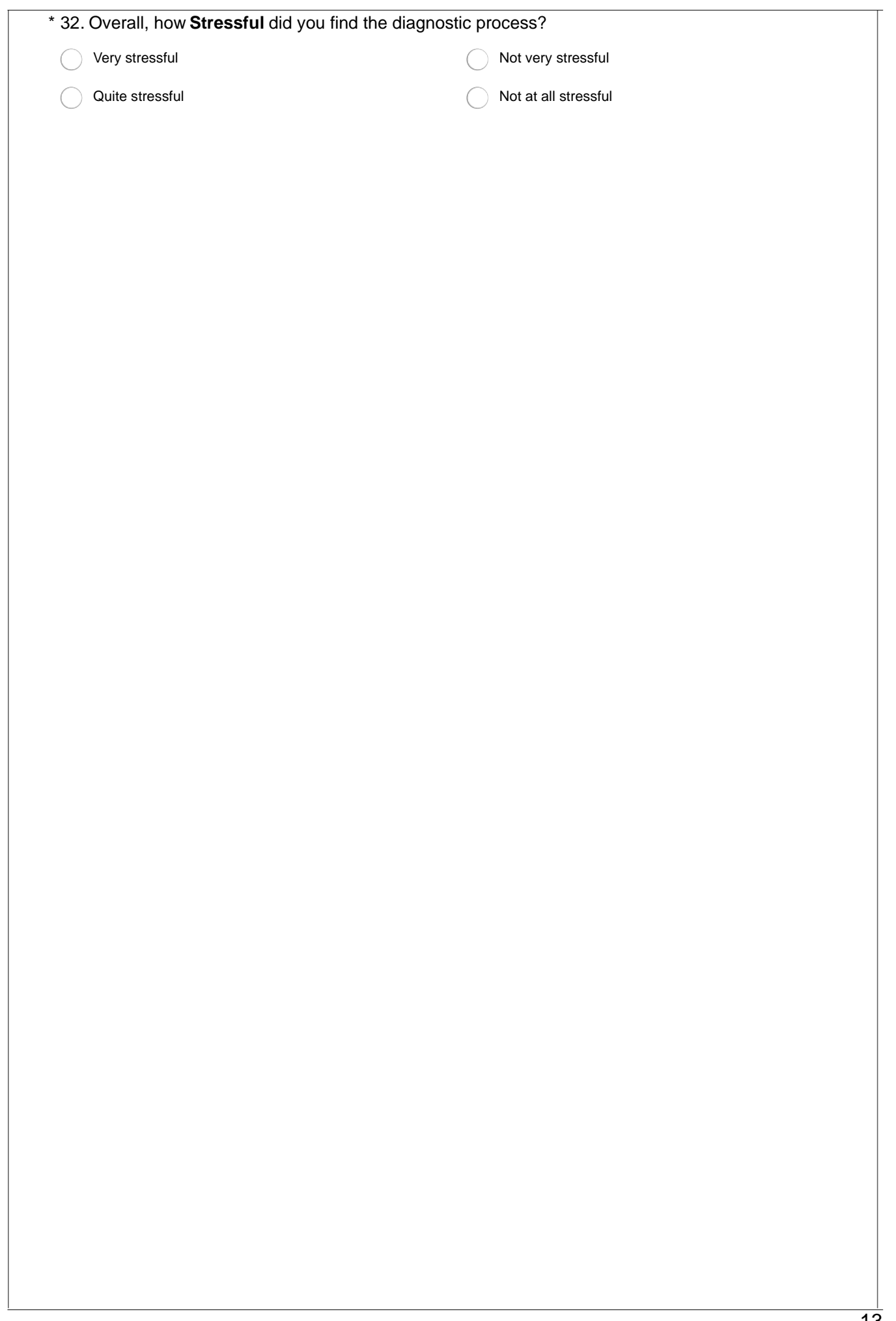


Exploring Parents'/Carers' Experience in Obtaining a Diagnosis of ASD for their Children and their Acceptance of Using an Online Diagnostics System (NODA)

\section{Section 5: Post-diagnostic Support}

* 33. What sort of help/support were you offered after your child was diagnosed? Please tick all that apply.
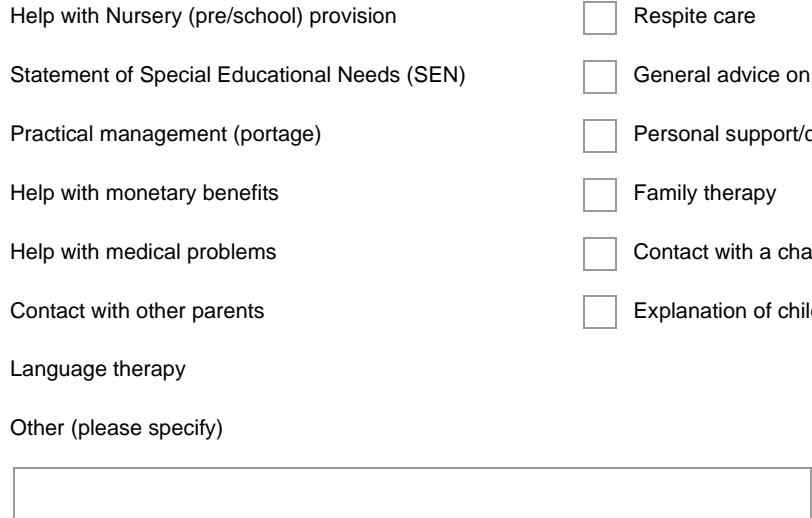

* 34. Overall, how satisfied were you with theHELP/SUPPORT OFFERED after your Child was diagnosed?
Very dissatisfied
Quite satisfied
Quite dissatisfied
Very satisfied
Neither satisfied nor dissatisfied 
Exploring Parents'/Carers' Experience in Obtaining a Diagnosis of ASD for their Children and their Acceptance of Using an Online Diagnostics System (NODA)

\section{Section 6: Access to services}

The next questions are about all the types of services children may need or use, such as diagnostic services, medical care, specialised therapy, counselling, special education, and early intervention. These services can be in clinics, schools, child care centres, through community programs, at home, and other places.

* 35 . Since [his/her] birth, did you need a referral to see any doctors or receive any services for your child ?
$\bigcirc$ Yes
Don't know

No

* 36 . Was getting referrals a big problem, a small problem, or not a problem?

Big problem

Not a problem

Small problem

Don't know

* 37 . Since [his/her] birth, did you have any difficulties or delays getting services for your child because [he/she] was not eligible for the services?

$\bigcirc$ Yes

Don't know

No

* 38 . Since [his/her] birth, did you have any difficulties or delays because of issues related to cost?

Yes

Don't know

No

* 39 . Since [his/her] birth, did you have any difficulties or delays because there were waiting lists, backlogs, or other problems getting appointments?

Yes

Don't know

No

* 40. Since [his/her] birth, did you have any difficulties or delays because the services for your child needed were not available in your area?

Yes

Don't know

No 


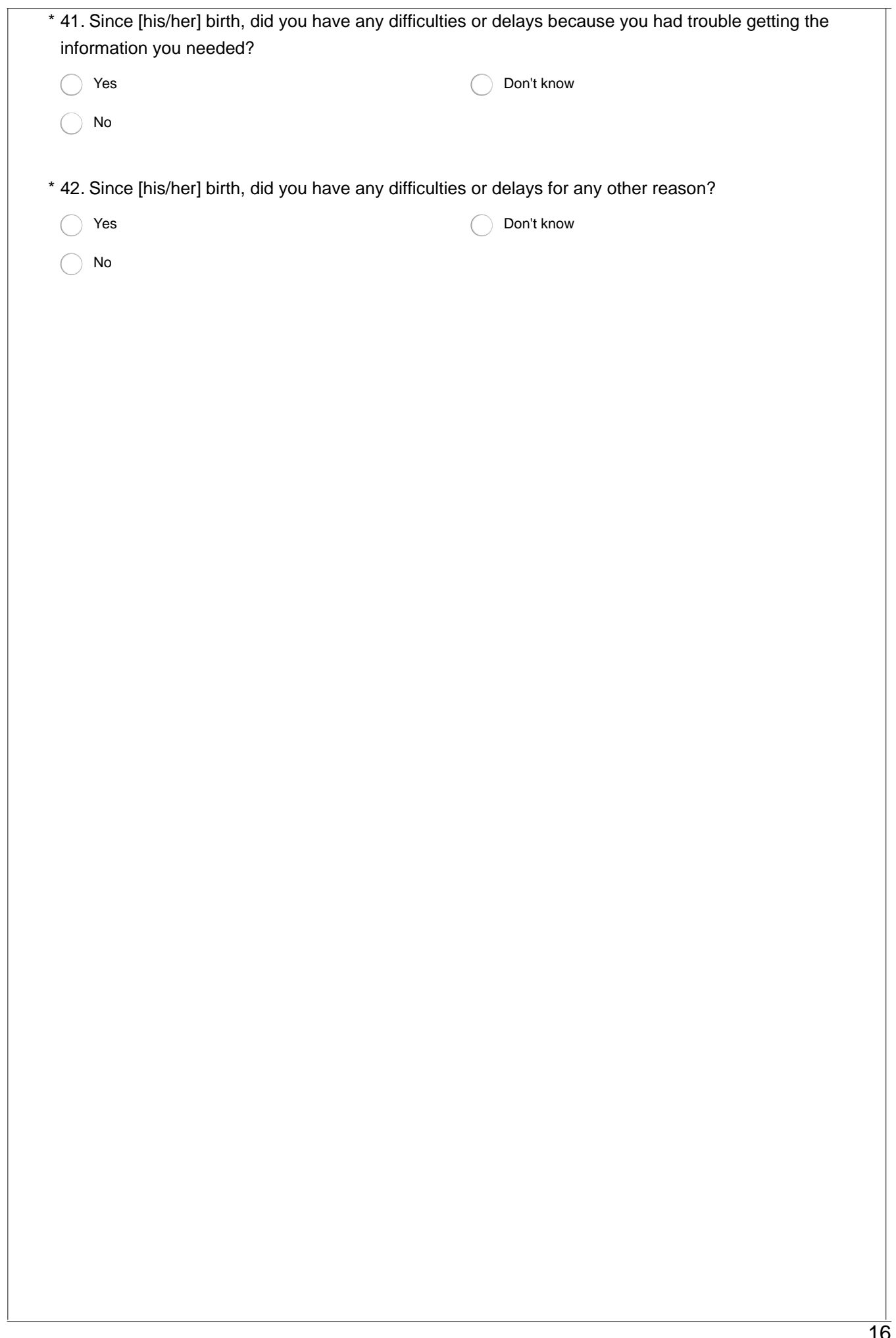


Exploring Parents'/Carers' Experience in Obtaining a Diagnosis of ASD for their Children and their Acceptance of Using an Online Diagnostics System (NODA)

* 43. If you answered Yes, Please specify? 
Exploring Parents'/Carers' Experience in Obtaining a Diagnosis of ASD for their Children and their Acceptance of Using an Online Diagnostics System (NODA)

* 44. Since [his/her] birth, how often have you been frustrated in your efforts to get services for your child?

Never

Always

Sometimes

Don't know

O Usually 
Exploring Parents'/Carers' Experience in Obtaining a Diagnosis of ASD for their Children and their Acceptance of Using an Online Diagnostics System (NODA)

PART (2) Parents'/Carers' acceptance of using an online ASD diagnostic system

Naturalistic Observation Diagnostic Assessment (NODA)

(Asynchronous Telehealth Remote Autism Diagnostic System)

Important Note: to understand the NODA system, please read the following information and see the short videos as shown below, before answering the survey questions.

\section{What is NODA?}

NODA is a Remote Autism Diagnostic System that guides parents to collect short videos of the child's behaviour at home and remotely share them with clinicians who conduct a diagnostic assessment for ASD without the need to have face to face communication. This system can facilitate sharing of both the current child's behaviour and developmental history with diagnostic professionals.

\section{What does NODA include?}

1. NODA Smart Capture: it is a smartphone app that enables parents to collect and share video evidence of their child's behavior at home (See Figures1-4 below). It allows parents to record and upload four 10-minute NODA scenarios. NODA scenarios include the child playing alone, the child playing with others, a family mealtime, and a behavior of parent's concern. The next part will show some short videos (each one around one minute) for sample videos and recording instructions for these scenarios.

2. NODA Connect: it is a web portal for diagnosticians to conduct a remote diagnostic assessment based on in-home videos, a brief developmental history and their clinical judgment. The resulting report can be shared with the parent (See Figure 5).

\section{Why NODA is developed?}

Research reveals that there is a long time between when parents first become concerned and receiving a diagnosis. This delay can hinder early interventions that have been noted to improve developmental outcomes. Therefore, NODA has been developed to reduce the time between parental concern and diagnosis. 


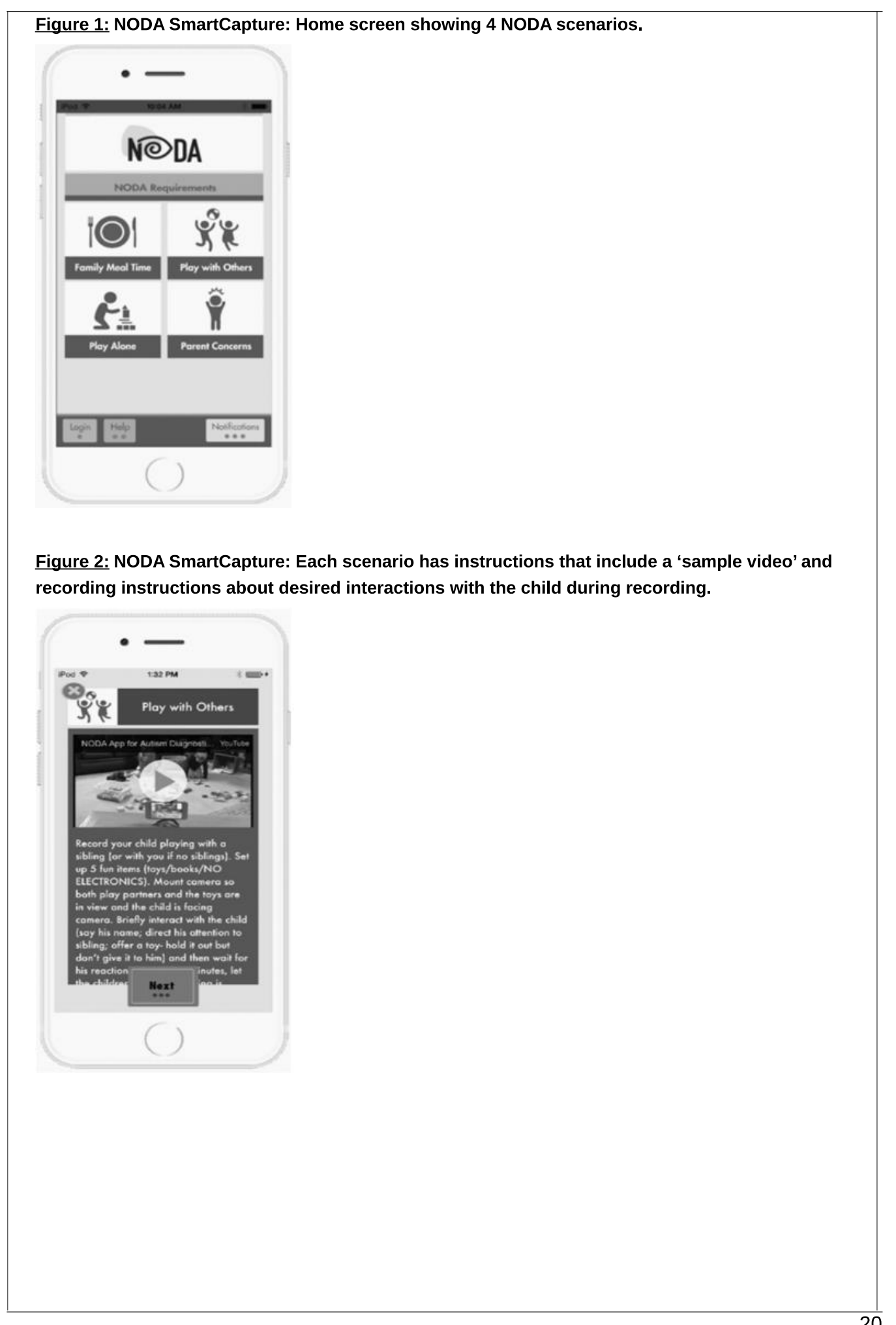


Figure 3: NODA SmartCapture: After going throughout instructions, parents can start video recording. Video can be stopped any time or it stops automatically after 10 minutes.

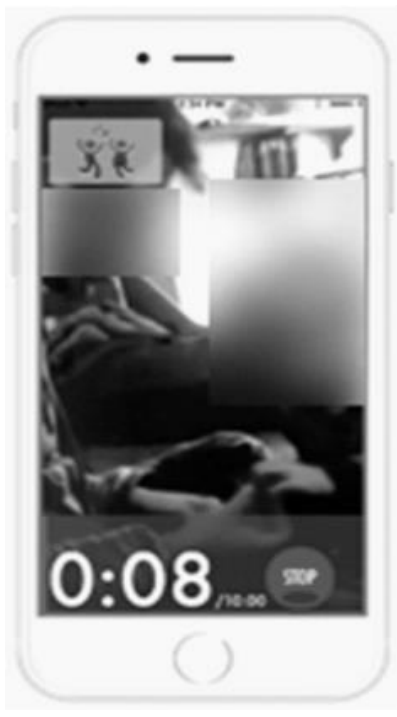

Figure 4: NODA SmartCapture: Home screen shows status of the recorded scenario with options to view, delete or send video to diagnostician. Once video is sent, it uploads to NODA Connect.

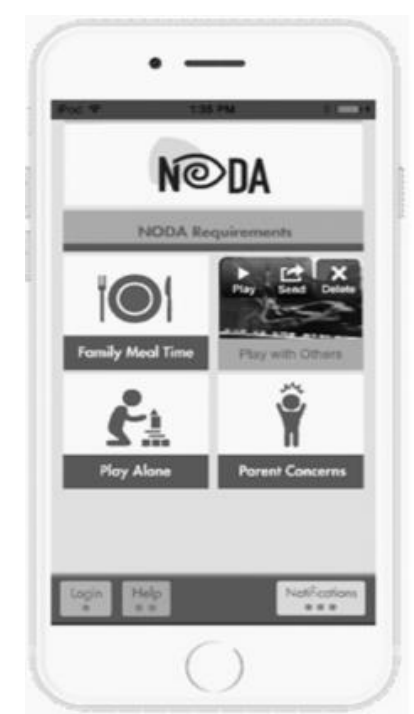


Figure 5: NODA Connect: Diagnostic report.

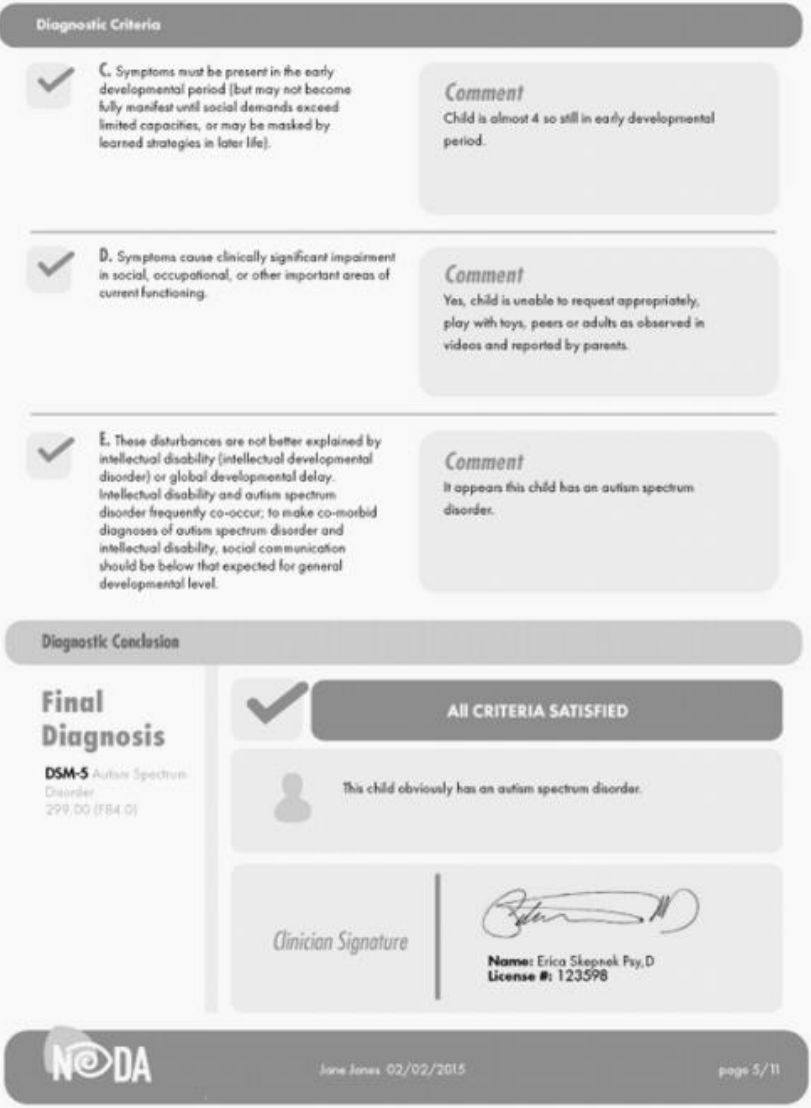

Please see the short videos as shown below before answering the survey questions (each one around one minute). 


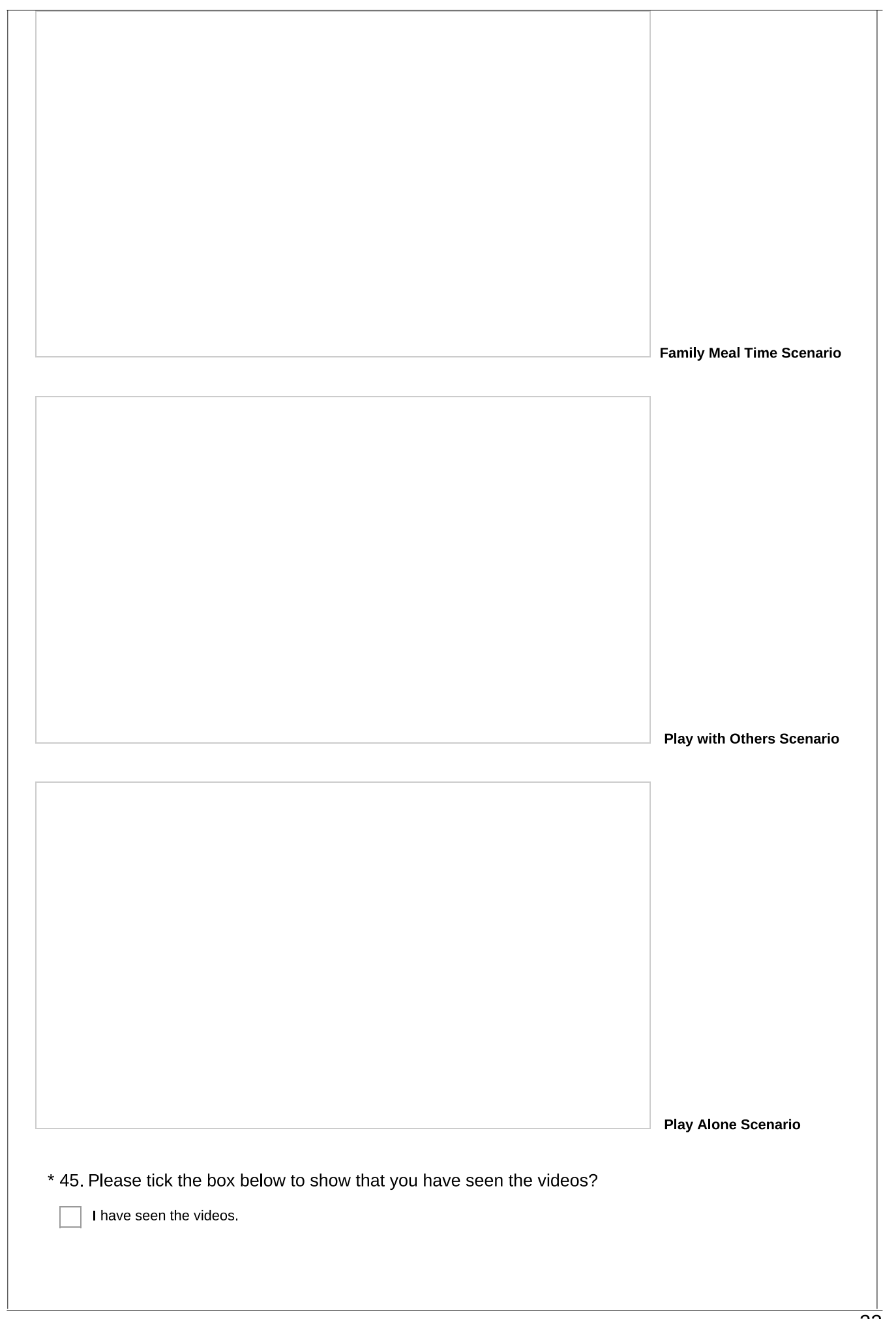


Exploring Parents'/Carers' Experience in Obtaining a Diagnosis of ASD for their Children and their Acceptance of Using an Online Diagnostics System (NODA)

\section{Section 1: Technology Experience}

* 46. How would you rate your computer/internet skills?
Novice ( none)
Advance
Basic
Expert
Average

* 47. How comfortable are you with using the following:

$\begin{array}{llll}\text { Very comfortable } & \begin{array}{r}\text { Somewhat } \\ \text { comfortable }\end{array} & \text { Natural } & \begin{array}{c}\text { Somewhat } \\ \text { uncomfortable }\end{array} \\ \text { Desktop Computer } & & & \end{array}$


Exploring Parents'/Carers' Experience in Obtaining a Diagnosis of ASD for their Children and their Acceptance of Using an Online Diagnostics System (NODA)

\section{Section 2: Your thoughts on using (NODA) for remote ASD diagnosis}

How would you answer the questions?

We know that your child has already been diagnosed with ASD. Therefore, we would like to explore your opinions about the NODA system. When answering the questionnaire below it is important to consider your answers, as if you had been asked these questions prior to receiving the diagnosis. Your views will be valuable and would help other parents who are trying to get a diagnosis for their children.

* 48. Here are (25) statements related to various factors that may be involved in your acceptance of using the NODA System for remote ASD diagnosis for your child. Please, indicate your level of agreement with each of the following statements.

\begin{tabular}{l} 
1. I would find the \\
NODA system useful in \\
the diagnosis of my \\
child. \\
2. Using the NODA \\
system would enable \\
me to receive the \\
diagnosis quickly. \\
3. Using the NODA \\
system to obtain a \\
diagnosis, for my child \\
would be productive in \\
accessing services. \\
4. If I use the NODA \\
system, it would \\
increase chances of \\
getting an earlier \\
diagnosis for my child. \\
5. From watching the \\
video, I think I would \\
have clear \\
understanding of how I \\
would interact with the \\
NODA system. \\
6. If I have training, I \\
think it would be easy \\
for me to become skilful \\
at using the NODA \\
system. \\
7. I think I would find \\
the NODA system easy \\
to use. \\
\hline
\end{tabular}




\begin{tabular}{|c|c|c|c|c|c|}
\hline & Strongly Agree & Agree & Disagree & Strongly Disagree & Don't know \\
\hline $\begin{array}{l}\text { 8. I think learning how } \\
\text { to use the NODA } \\
\text { system would be easy } \\
\text { for me. }\end{array}$ & & & & & \\
\hline $\begin{array}{l}\text { 9. My family members } \\
\text { would think that I } \\
\text { should use the NODA } \\
\text { system. }\end{array}$ & & & & & \\
\hline $\begin{array}{l}\text { 10. My friends would } \\
\text { think that I should use } \\
\text { the NODA system. }\end{array}$ & & & & & \\
\hline $\begin{array}{l}\text { 11. I would like to think } \\
\text { that professionals } \\
\text { would support the use } \\
\text { of the NODA system } \\
\text { (e.g. GP, physiologists, } \\
\text { health professionals, } \\
\text { teachers). }\end{array}$ & & & & & \\
\hline $\begin{array}{l}\text { 12. I would have the } \\
\text { resources necessary to } \\
\text { use the NODA system } \\
\text { (e.g. internet, computer, } \\
\text { smart phone, iPad). }\end{array}$ & & & & & \\
\hline $\begin{array}{l}\text { 13. I would have the } \\
\text { knowledge necessary } \\
\text { to use the NODA } \\
\text { system. }\end{array}$ & & & & & \\
\hline $\begin{array}{l}\text { 14. I think my family } \\
\text { and friends would be } \\
\text { available for assistance } \\
\text { with NODA system } \\
\text { difficulties. }\end{array}$ & & & & & \\
\hline $\begin{array}{l}\text { 15. I think I could } \\
\text { collect and share video } \\
\text { evidence of my child's } \\
\text { behaviour using the } \\
\text { NODA system on my } \\
\text { own. }\end{array}$ & & & & & \\
\hline $\begin{array}{l}\text { 16. I think I could } \\
\text { collect and share video } \\
\text { evidence of my child's } \\
\text { behaviour using the } \\
\text { NODA system if I could } \\
\text { call someone for help if } \\
\text { I got stuck (e.g. Help } \\
\text { line). }\end{array}$ & & & & & \\
\hline
\end{tabular}




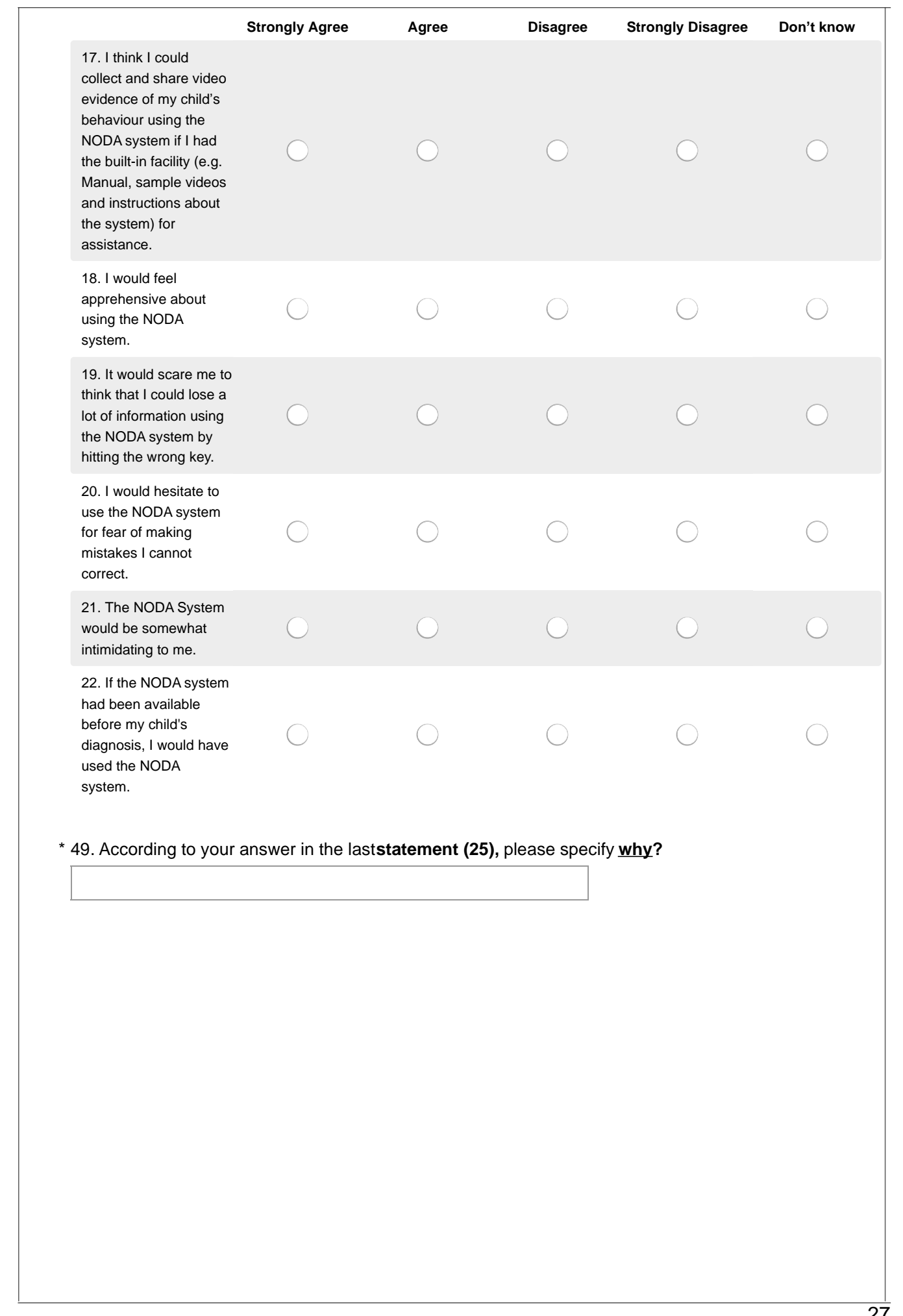


Exploring Parents'/Carers' Experience in Obtaining a Diagnosis of ASD for their Children and their Acceptance of Using an Online Diagnostics System (NODA)

\section{Section 3}

* 50. Would you have considered using the NODA system to receive a diagnosis for your child if it had been offered free by the NHS?
Yes
I don't know
No

51. Would you have considered using the NODA system to receive a diagnosis for your child if it had been offered privately and accepted by the NHS as an official diagnosis, (the price would be around $£ 180)$ ?

Yes

No

I don't know

52. If you wish to be entered into the prize draw, please provide your email in the box below. 
Exploring Parents'/Carers' Experience in Obtaining a Diagnosis of ASD for their Children and their Acceptance of Using an Online Diagnostics System (NODA)

Debriefing Form

Thank you for your participation in this study. The data will be used for research purposes only and all information produced by this study will be anonymous. All participants will be treated confidentially, and all information will be stored securely, thus, protecting privacy, confidentiality and anonymity.

If you would like to receive a copy of the results at the end of the study, please contact:

If your participation in this study has caused you some concerns, anxiety, or distress, you may contact NAS Helpline (08088004104).

If you have any further questions about the study, please contact: Manahil Alfuraydan, Researcher ), or

Dr Jodie Croxall, PhD research supervisor

, or

Prof. Sinead Brophy, PhD research supervisor

THANK YOU AGAIN FOR YOUR PARTICIPATION. 


\section{Appendix 8: Ethical Approval}

\section{Ethical Approval}

Ethics Committee Use Only

\begin{tabular}{|l|l|}
\hline $\begin{array}{l}\text { Principal } \\
\text { Investigator }\end{array}$ & Manahil Alfuraydan \\
\hline $\begin{array}{l}\text { Title of Proposed } \\
\text { Research }\end{array}$ & $\begin{array}{l}\text { Exploring Parents'/Carers' Experience in Obtaining a Diagnosis of ASD } \\
\text { for their Children and their Acceptance of Using Online Diagnostics } \\
\text { System (NODA) in Wales }\end{array}$ \\
\hline $\begin{array}{l}\text { RESC Project } \\
\text { reference number }\end{array}$ & 2018-0013A \\
\hline
\end{tabular}

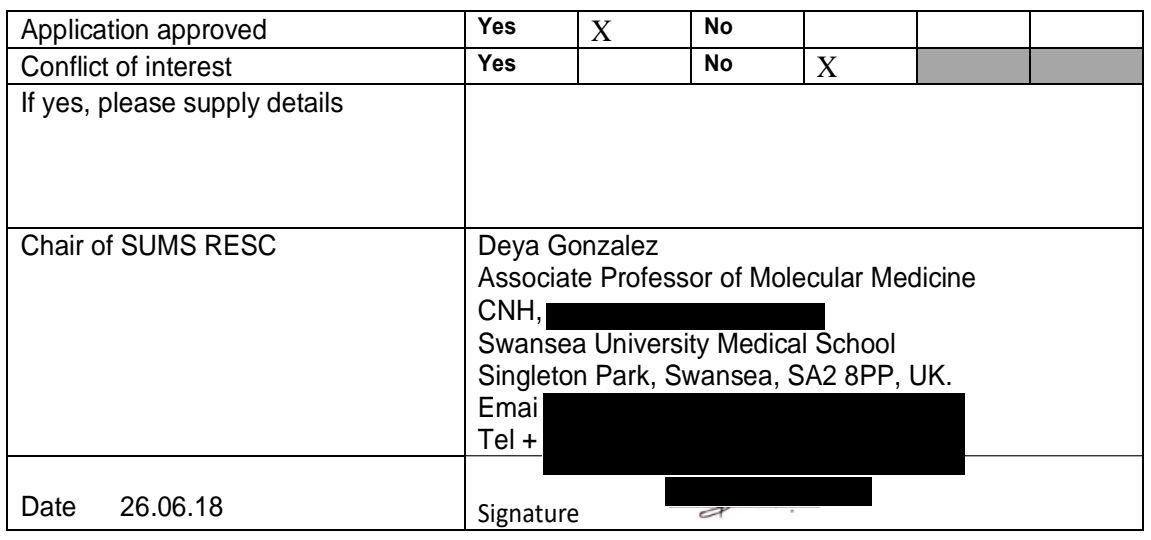

This application has been granted ethical approval in its current form.

Please ensure that you quote project reference number 2018-0013A in any correspondence with the SUMS RESC

\begin{tabular}{|l|l|}
\hline Time limit for applicant to respond & $\begin{array}{l}\text { (two months from receipt of email from ethics } \\
\text { panel) }\end{array}$
\end{tabular}




\section{References}

Aboujaoude, E., Salame, W., \& Naim, L. (2015). Telemental health: a status update. World Psychiatry, 14(2), 223-230.

Adenuga, K. I., Iahad, N. A., \& Miskon, S. (2017). Towards reinforcing telemedicine adoption amongst clinicians in Nigeria. International Journal of Medical Informatics, 104, 84-96.

Agarwal, R., \& Prasad, J. (1999). Are individual differences germane to the acceptance of new information technologies? Decision Sciences, 30(2), 361-391.

Ajibade, P. (2018). Technology acceptance model limitations and criticisms: Exploring the practical applications and use in technology-related studies, mixed-method, and qualitative researches. Library Philosophy and Practice, 1941, 1-14.

Ajzen, I. (1985). From Intentions to actions: A theory of planned behaviour. In J. Kuhl \& J. Beckman (Eds.), Action-control: From cognition to behaviour (pp. 1139). Heidelberg: Springer.

Ajzen, I. (1991). The theory of planned behaviour. Organisational Behaviour and Human Decision Processes, 50(2), 179-211.

Ajzen, I., \& Fishbein, M. (1980). Understanding attitudes and predicting social behaviour. Englewood Cliffs: Prentice-Hall.

Ajzen, I., \& Fishbein, M. (2005). The influence of attitudes on behaviour. In D. Albarracín, B. T. Johnson, \& M. P. Zanna (Eds.), The handbook of attitudes (pp. 173-221). New York: Lawrence Erlbaum Associates.

Al-Safadi, L. (2016). The Effects of Real-Time Interactive Multimedia Teleradiology System. BioMed Research International, 2016, 1-9. 
Albrecht, U.-V., Afshar, K., Illiger, K., Becker, S., Hartz, T., Breil, B., . . von Jan, U. (2017). Expectancy, usage and acceptance by general practitioners and patients: exploratory results from a study in the German outpatient sector. Digital Health, 3, 1-22.

Alkhalifah, S., \& Aldhalaan, H. (2018). Telehealth services for children with autism spectrum disorders in rural areas of the Kingdom of Saudi Arabia: Overview and recommendations. JMIR Pediatrics and Parenting, 1(2), 1-7.

Alvseike, H., \& Brønnick, K. (2012). Feasibility of the iPad as a hub for smart house technology in the elderly; effects of cognition, self-efficacy, and technology experience. Journal of Multidisciplinary Healthcare, 5, 299-306.

American Psychiatric Association. (2000). Diagnostic and Statistical Manual of Mental Disorders $(D S M-I V-R T)$ (4th ed.). Washington, DC: American Psychiatric Association.

American Psychiatric Association. (2013a). Diagnostic and statistical manual of mental disorders $\left(5^{\text {th }}\right.$ ed.). Arlington, VA: American Psychiatric Association.

American Psychiatric Association. (2013b). What is Autism Spectrum Disorder? Retrieved from https://www.psychiatry.org/patients-families/autism/what-isautism-spectrum-disorder

American Telemedicine Association. (2017). About Telemedicine. Retrieved May 15, 2016, from http://www.americantelemed.org/about/about-telemedicine

Anguera, M. T., Blanco-Villaseñor, A., Losada, J. L., Sánchez-Algarra, P., \& Onwuegbuzie, A. J. (2018). Revisiting the difference between mixed methods and multimethods: Is it all in the name? Quality \& Quantity, 52(6), 2757-2770. 
Aresti-Bartolome, N., \& Garcia-Zapirain, B. (2014). Technologies as support tools for persons with autistic spectrum disorder: A systematic review. International Journal of Environmental Research and Public Health, 11(8), 7767-7802.

Arksey, H., \& O'Malley, L. (2005). Scoping studies: Towards a methodological framework. International Journal of Social Research Methodology, 8(1), 1932.

Attuquayefio, S., \& Addo, H. (2014). Review of studies with UTAUT as conceptual framework. European Scientific Journal, 10(8), 249-258.

Baghdadli, A., Picot, M. C., Pascal, C., Pry, R., \& Aussilloux, C. (2003). Relationship between age of recognition of first disturbances and severity in young children with autism. European Child \& Adolescent Psychiatry, 12(3), 122-127.

Bandura, A. (1989). Human agency in social cognitive theory. American Psychologist, 44(9), 1175-1184.

Bashshur, R. L., Bashshur, R., Shannon, G. W., \& Sanders, J. H. (1997). Telemedicine: Theory and practice. Springfield: Charles C. Thomas.

Baudouin, S. J. (2014). Heterogeneity and convergence: The synaptic pathophysiology of autism. European Journal of Neuroscience, 39(7), 1107-1113.

Bawack, R. E., \& Kala Kamdjoug, J. R. (2018). Adequacy of UTAUT in clinician adoption of health information systems in developing countries: The case of Cameroon. International Journal of Medical Informatics, 109, 15-22.

Berkowsky, R. W., Sharit, J., \& Czaja, S. J. (2018). Factors predicting decisions about technology adoption among older adults. Innovation in Aging, 1(3), 1-12.

Bewick, V., Cheek, L., \& Ball, J. (2005). Statistics review 14: Logistic regression. Critical Care, 9(1), 112-118. 
Boisvert, M., \& Hall, N. (2014). The use of telehealth in early autism training for parents: a scoping review. Smart Homecare Technology and Telehealth, 2, 1927.

Boisvert, M., Lang, R., Andrianopoulos, M., \& Boscardin, M. L. (2010). Telepractice in the assessment and treatment of individuals with autism spectrum disorders: A systematic review. Developmental Neurorehabilitation, 13(6), 423-432.

Book, L. A. (2009). Early Red Flags for Autism Spectrum Disorders in Toddlers in the Home Environment. (Doctoral dissertation ). Retrieved from Florida State University Digital repository database.

Boulet, S. L., Boyle, C. A., \& Schieve, L. A. (2009). Health care use and health and functional impact of developmental disabilities among US children, 19972005. Archives of Pediatrics and Adolescent Medicine, 163(1), 19-26.

Bowman, R., Kennedy, C., Kirwan, J., Sze, P., \& Murdoch, I. (2003). Reliability of telemedicine for diagnosing and managing eye problems in accident and emergency departments. Eye, 17(6), 743.

Braun, V., \& Clarke, V. (2006). Using thematic analysis in psychology. Qualitative Research in Psychology, 3(2), 77-101.

Breil, B., Kremer, L., Hennemann, S., \& Apolinário-Hagen, J. (2019). Acceptance of mHealth apps for self-management among people with hypertension. Studies in Health Technology and Informatics, 267, 282-288.

Brewer, J., \& Hunter, A. (1989). Multimethod Research: A synthesis of styles. Thousand Oaks: Sage Publications, Inc.

Brewer, J., \& Hunter, A. (2006). Foundations of multimethod research: Synthesizing styles. Thousand Oaks: Sage Publications, Inc. 
Brunetti, N. D., Scalvini, S., Acquistapace, F., Parati, G., Volterrani, M., Fedele, F., \& Molinari, G. (2015). Telemedicine for cardiovascular disease continuum: a position paper from the Italian Society of Cardiology Working Group on Telecardiology and Informatics. International Journal of Cardiology, 184, $452-458$

Bryman, A. (2012). Social Reserach Methods (4 ${ }^{\text {th }}$ ed.). New York: Oxford University Press.

Bursac, Z., Gauss, C. H., Williams, D. K., \& Hosmer, D. W. (2008). Purposeful selection of variables in logistic regression. Source Code for Biology and Medicine, 3(1), 1-8.

Caffery, L. J., Farjian, M., \& Smith, A. C. (2016). Telehealth interventions for reducing waiting lists and waiting times for specialist outpatient services: A scoping review. Journal of Telemedicine and Telecare, 22(8), 504-512.

Carr, E. G., \& Durand, V. M. (1985). Reducing behaviour problems through functional communication training. Journal of Applied Behaviour Analysis, 18(2), 111126.

Centers for Disease Control and Prevention. (2011). National Survey of Children with Special Health Care Needs. Retrieved from https://www.cdc.gov/nchs/slaits/cshen.htm

Centers for Disease Control and Prevention. (2018). Data and Statistics on Autism Spectrum Disorder. Retrieved from https://www.cdc.gov/ncbddd/autism/data.html

Centers for Disease Control and Prevention. (2019). Signs and Symptoms of Autism Spectrum Disorders. Retrieved from https://www.cdc.gov/ncbddd/autism/signs.html 
Chamak, B., Bonniau, B., Oudaya, L., \& Ehrenberg, A. (2011). The autism diagnostic experiences of French parents. Autism, 15(1), 83-97.

Chang, S. J., \& Im, E.-O. (2014). A path analysis of Internet health information seeking behaviours among older adults. Geriatric Nursing, 35(2), 137-141.

Charman, T., \& Gotham, K. (2013). Measurement Issues: Screening and diagnostic instruments for autism spectrum disorders-lessons from research and practise. Child and Adolescent Mental Health, 18(1), 52-63.

Chau, P. Y., \& Hu, P. J. H. (2001). Information technology acceptance by individual professionals: A model comparison approach. Decision Sciences, 32(4), 699719.

Chedid, R. J., Dew, A., \& Veitch, C. (2013). Barriers to the use of Information and Communication Technology by occupational therapists working in a rural area of New South Wales, Australia. Australian Occupational Therapy Journal, 60(3), 197-205.

Choo, D., Dettman, S., Dowell, R., \& Cowan, R. (2019). Wearable Technology to Support Early Child Language Experiences: What's Important to Parents and Clinicians? Studies in Health Technology and Informatics, 266, 51-56.

Chua, S. L., Chen, D.-T., \& Wong, A. F. (1999). Computer anxiety and its correlates: a meta-analysis. Computers in Human Behaviour, 15(5), 609-623.

Cimperman, M., Brenčič, M. M., \& Trkman, P. (2016). Analyzing older users' home telehealth services acceptance behaviour-applying an Extended UTAUT model. International Journal of Medical Informatics, 90, 22-31.

Clarke, V., \& Braun, V. (2013). Teaching thematic analysis: Overcoming challenges and developing strategies for effective learning. The Psychologist, 26(2), 120123. 
Cohen, L., Manion, L., \& Morrison, k. (2007). Reserach Methods in Education (6 ${ }^{\text {th }}$ ed.). New York: Routledge.

Corsello, C. M. (2005). Early intervention in autism. Infants \& Young Children, 18(2), $74-85$.

Crane, L., Chester, J. W., Goddard, L., Henry, L. A., \& Hill, E. (2015). Experiences of autism diagnosis: A survey of over 1000 parents in the United Kingdom. Autism, 20(2), 153-162.

Cranen, K., Drossaert, C. H. C., Brinkman, E. S., Braakman-Jansen, A. L. M., Ijzerman, M. J., \& Vollenbroek-Hutten, M. M. R. (2012). An exploration of chronic pain patients' perceptions of home telerehabilitation services. Health Expectations: An International Journal of Public Participation in Health Care and Health Policy, 15(4), 339-350.

Creswell, J. W. (2009). Research Design Qualitative, Quantitative, and Mixed Methods Approaches. ( ${ }^{\text {rd }}$ ed.). Thousand Oaks: SAGE.

Creswell, J. W. (2014). Research Design Qualitative, Quantitative, \& Mixed Methods Approches ( $4^{\text {th }}$ ed.). Thousand Oaks: SAGE.

Critical Appraisal Skills Programme. (2018). CASP Checklists. Retrieved from https://casp-uk.net/casp-tools-checklists/

Czaja, S. J., Charness, N., Fisk, A. D., Hertzog, C., Nair, S. N., Rogers, W. A., \& Sharit, J. (2006). Factors predicting the use of technology: findings from the Center for Research and Education on Aging and Technology Enhancement (CREATE). Psychology and Aging, 21(2), 333-352.

Dai, B., Larnyo, E., Tetteh, E. A., Aboagye, A. K., \& Musah, A.-A. I. (2020). Factors Affecting Caregivers' Acceptance of the Use of Wearable Devices by Patients With Dementia: An Extension of the Unified Theory of Acceptance and Use 
of Technology Model. American Journal of Alzheimer's Disease \& Other Dementias, 35, 1-11.

Daniel, J. (2012). Sampling Essentials: Practical Guidelines for Making Sampling

Choices. Retrieved from http://methods.sagepub.com/book/samplingessentials.

Daniels, A. M., Como, A., Hergüner, S., Kostadinova, K., Stosic, J., \& Shih, A. (2017). Autism in Southeast Europe: A Survey of Caregivers of Children with Autism Spectrum Disorders. Journal of Autism and Developmental Disorders, 47(8), 2314-2325.

Davidovitch, M., Glick, L., Holtzman, G., Tirosh, E., \& Safir, M. P. (2000). Developmental regression in autism: maternal perception. Journal of Autism and Developmental Disorders, 30(2), 113-119.

Davies, A., Sharp, C., Homolova, L., \& Bellis, M. (2019). Population health in a digital age: The use of digital technology to support and monitor health in Wales. Retrieved from https://phw.nhs.wales/files/research/population-healthin-a-digital-age/

Davis, F. D. (1989). Perceived usefulness, perceived ease of use, and user acceptance of information technology. Management Information Systems Quarterly, 13(3), 319-340.

Davis, F. D. (1993). User acceptance of information technology: system characteristics, user perceptions and behavioural impacts. International Journal of Man-Machine Studies, 38(3), 475-487.

Davis, F. D., Bagozzi, R. P., \& Warshaw, P. R. (1989). User acceptance of computer technology: a comparison of two theoretical models. Management Science, 35(8), 982-1003. 
Davis, F. D., Bagozzi, R. P., \& Warshaw, P. R. (1992). Extrinsic and intrinsic motivation to use computers in the workplace. Journal of Applied Social Psychology, 22(14), 1111-1132.

Dawson, G., Rogers, S., Munson, J., Smith, M., Winter, J., Greenson, J., . . V Varley, J. (2010). Randomized, controlled trial of an intervention for toddlers with autism: the Early Start Denver Model. Pediatrics, 125(1), e17-e23.

De Graaf, M., Totté, J. E., Van Os-Medendorp, H., Van Renselaar, W., Breugem, C. C., \& Pasmans, S. G. (2014). Treatment of infantile hemangioma in regional hospitals with eHealth support: evaluation of feasibility and acceptance by parents and doctors. JMIR Research Protocols, 3(4), e52.

De Vaus, D. A., \& de Vaus, D. (2001). Research design in social research. London: Sage.

Dillman, D. A., Smyth, J. D., \& Christian, L. M. (2014). Internet, phone, mail, and mixed-mode surveys: The tailored design method ( $4^{\text {th }}$ ed.). New Jersey: John Wiley \& Sons.

Dillon, A. (2001). User acceptance of information technology. In W. Karwowski (Ed.), Encyclopedia of Human Factors and Ergonomics. London: Taylor and Francis.

Dillon, A., \& Morris, M. (1996). User acceptance of new information technology theories and models. In M. Williams (Ed.), Annual Review of Information Science and Technology (pp. 3-32). Medford NJ: Information Today.

Dou, K., Yu, P., Deng, N., Liu, F., Guan, Y., Li, Z., . . . Duan, H. (2017). Patients' acceptance of smartphone health technology for chronic disease management: A theoretical model and empirical test. JMIR Mhealth Uhealth, 5(12), e177. 
Dugger, C. E. (2012). The Effects of Early Intervention on Children with Autism Spectrum Disorders. (Master's thesis). Retrieved from Southern Illinois University digital repository database.

Dunkley, C., Pattie, L., Wilson, L., \& McAllister, L. (2010). A comparison of rural speech-language pathologists' and residents' access to and attitudes towards the use of technology for speech-language pathology service delivery. International Journal of Speech-Language Pathology, 12(4), 333-343.

Dunn, B. E., Choi, H., Almagro, U. A., Recla, D. L., \& Davis, C. W. (2000). Telepathology networking in VISN-12 of the Veterans Health Administration. Telemedicine Journal and e-Health, 6(3), 349-354.

Eggleston, M. J., Thabrew, H., Frampton, C. M., Eggleston, K. H., \& Hennig, S. C. (2019). Obtaining an autism spectrum disorder diagnosis and supports: New Zealand parents' experiences. Research in Autism Spectrum Disorders, 62, 1825.

Eichenberg, C., Wolters, C., \& Brähler, E. (2013). The internet as a mental health advisor in Germany—results of a national survey. PloS one, 8(11).

Esmaeilzadeh, P., Sambasivan, M., Kumar, N., \& Nezakhati, H. (2011). Adoption of technology applications in healthcare: The influence of attitude toward knowledge sharing on technology acceptance in a hospital. In T. Kim. et al. (Eds), Procedings of the International Conference on U-and E-Service, Science and Technology: Vol. 264. Communication in Computer and Information Scince (pp. 17-30). Berlin: Springer.

Falkmer, T., Anderson, K., Falkmer, M., \& Horlin, C. (2013). Diagnostic procedures in autism spectrum disorders: a systematic literature review. European Child \& Adolescent Psychiatry, 22(6), 329-340. 
Fatehi, F., \& Wootton, R. (2012). Telemedicine, telehealth or e-health? A bibliometric analysis of the trends in the use of these terms. Journal of Telemedicine and Telecare, 18(8), 460-464.

Fichman, R. G., Kohli, R., \& Krishnan, R. (2011). Editorial overview-the role of information systems in healthcare: current research and future trends. Information Systems Research, 22(3), 419-428.

Field, A. (2005). Discovering statistics using SPSS (2 ${ }^{\text {nd }}$ ed.). London: Sage Publications, Inc.

Field, A. (2013). Discovering statistics using IBM SPSS statistics (4 ${ }^{\text {th }}$ ed.). London: Sage.

Fishbein, M., \& Ajzen, I. (1975). Belief, attitude, Intention and Behaviour: An Introduction to Theory and Research. Reading, Mass: Addison-Wesley Pub. Co.

Garavand, A., Mohseni, M., Asadi, H., Etemadi, M., Moradi-Joo, M., \& Moosavi, A. (2016). Factors influencing the adoption of health information technologies: a systematic review. Electronic Physician, 8(8), 2713-2718.

Garson, G. D. (2015). Missing Values Analysis and Data Imputation. Asheboro, NC: Statistical Associates Publishers.

Ghazizadeh, M., Lee, J. D., \& Boyle, L. N. (2012). Extending the Technology Acceptance Model to assess automation. Cognition, Technology \& Work, 14(1), 39-49.

Gillberg, C., Nordin, V., \& Ehlers, S. (1996). Early detection of autism. Diagnostic instruments for clinicians. European Child \& Adolescent Psychiatry, 5(2), 6774.

Gillham, B. (2008). Developing a questionnaire (2 ${ }^{\text {nd }}$ ed.). London: Continuum. 
Glass, C. R., \& Knight, L. A. (1988). Cognitive factors in computer anxiety. Cognitive Therapy and Research, 12(4), 351-366.

Goin-Kochel, R. P., Mackintosh, V. H., \& Myers, B. J. (2006). How many doctors does it take to make an autism spectrum diagnosis? Autism, 10(5), 439-451.

Goodman, R., Ford, T., Richards, H., Gatward, R., \& Meltzer, H. (2000). The development and well-being assessment: Description and initial validation of an integrated assessment of child and adolescent psychopathology. Journal of Child Psychology and Psychiatry, 41(5), 645-655.

Gottfried, C., Bambini-Junior, V., Francis, F., Riesgo, R., \& Savino, W. (2015). The impact of neuroimmune alterations in autism spectrum disorder. Frontiers In Psychiatry, 6, 1-16.

Granić, A., \& Marangunić, N. (2019). Technology acceptance model in educational context: A systematic literature review. British Journal of Educational Technology, 50(5), 2572-2593.

Greenhalgh, T., Robert, G., Bate, P., Macfarlane, F., \& Kyriakidou, O. (2005). Diffusion of innovations in health service organisations: a systematic literature review. Malden, Mass: Blackwell.

Gros, D. F., Morland, L. A., Greene, C. J., Acierno, R., Strachan, M., Egede, L. E., . . . Frueh, B. C. (2013). Delivery of evidence-based psychotherapy via video telehealth. Journal of Psychopathology and Behavioural Assessment, 35(4), 506-521.

Guljas, R., Ahmed, A., Chang, K., \& Whitlock, A. (2014). Impact of telemedicine in managing type 1 diabetes among school-age children and adolescents: an integrative review. Journal of Pediatric Nursing, 29(3), 198-204. 
Guo, X., Sun, Y., Wang, N., Peng, Z., \& Yan, Z. (2013). The dark side of elderly acceptance of preventive mobile health services in China. Electronic Markets, 23(1), 49-61.

Hansen, S. N., Schendel, D. E., \& Parner, E. T. (2015). Explaining the increase in the prevalence of autism spectrum disorders: the proportion attributable to changes in reporting practices. JAMA Pediatrics, 169(1), 56-62.

Happé, F., \& Ronald, A. (2008). The 'fractionable autism triad': a review of evidence from behavioural, genetic, cognitive and neural research. Neuropsychology Review, 18(4), 287-304.

Harst, L., Lantzsch, H., \& Scheibe, M. (2019). Theories predicting end-user acceptance of telemedicine use: systematic review. Journal of Medical Internet Research, 21(5), 1-19.

Hasnat, M., \& Graves, P. (2000). Disclosure of developmental disability: A study of parent satisfaction and the determinants of satisfaction. Journal of Paediatrics and Child Health, 36(1), 32-35.

Hayes, S. A., \& Watson, S. L. (2013). The impact of parenting stress: A meta-analysis of studies comparing the experience of parenting stress in parents of children with and without autism spectrum disorder. Journal of Autism and Developmental Disorders, 43(3), 629-642.

Hennemann, S., Beutel, M. E., \& Zwerenz, R. (2016). Drivers and Barriers to Acceptance of Web-Based Aftercare of Patients in Inpatient Routine Care: A Cross-Sectional Survey. Journal of Medical Internet Research, 18(12), 1-17.

Hennemann, S., Witthöft, M., Bethge, M., Spanier, K., Beutel, M. E., \& Zwerenz, R. (2018). Acceptance and barriers to access of occupational e-mental health: cross-sectional findings from a health-risk population of employees. 
International Archives of Occupational and Environmental Health, 91(3), 305316.

Hesse-Biber, S. N., \& Johnson, R. B. (2015). The Oxford handbook of multimethod and mixed methods research inquiry. Oxford: Oxford University Press.

Hilty, D. M., Ferrer, D. C., Parish, M. B., Johnston, B., Callahan, E. J., \& Yellowlees, P. M. (2013). The effectiveness of telemental health: a 2013 review. Telemedicine and e-Health, 19(6), 444-454.

Hinton, P. R., McMurray, I., \& Brownlow, C. (2004). SPSS explained. London: Routledge Taylor \& Francis Group

Hollier, J. M., Vaughan, A. O., Liu, Y., van Tilburg, M. A., Shulman, R. J., \& Thompson, D. I. (2018). Maternal and Child Acceptability of a Proposed Guided Imagery Therapy Mobile App Designed to Treat Functional Abdominal Pain Disorders in Children: Mixed-Methods Predevelopment Formative Research. JMIR Pediatrics and Parenting, 1(1), 1-16.

Hoogenbosch, B., Postma, J., de Man-van Ginkel, J. M., Tiemessen, N. A., van Delden, J. J., \& van Os-Medendorp, H. (2018). Use and the Users of a Patient Portal: Cross-Sectional Study. Journal Medical Internet Research, 20(9), e262. doi:10.2196/jmir.9418

Hoque, R., \& Sorwar, G. (2017). Understanding factors influencing the adoption of mHealth by the elderly: An extension of the UTAUT model. International Journal of Medical Informatics, 101, 75-84.

Howlin, P., \& Asgharian, A. (1999). The diagnosis of autism and Asperger syndrome: findings from a survey of 770 families. Developmental Medicine \& Child Neurology, 41(12), 834-839. 
Howlin, P., \& Moore, A. (1997). Diagnosis in autism: A survey of over 1200 patients in the UK. Autism, 1(2), 135-162.

Hsieh, J.-C., Li, A.-H., \& Yang, C.-C. (2013). Mobile, cloud, and big data computing: contributions, challenges, and new directions in telecardiology. International Journal of Environmental Research and Public Health, 10(11), 6131-6153.

Hu, P. J., Chau, P. Y., Sheng, O. R. L., \& Tam, K. Y. (1999). Examining the technology acceptance model using physician acceptance of telemedicine technology. Journal of Management Information Systems, 16(2), 91-112.

Hurt, L., Langley, K., North, K., Southern, A., Copeland, L., Gillard, J., \& Williams, S. (2019). Understanding and improving the care pathway for children with autism. International Journal Health Care Quality Assurance, 32(1), 208-223.

Hyman, S. L., Rodier, P. M., \& Davidson, P. (2001). Pervasive developmental disorders in young children. Journal of the American Medical Association, 285(24), 3141-3142.

Iacono, T., Dissanayake, C., Trembath, D., Hudry, K., Erickson, S., \& Spong, J. (2016). Family and practitioner perspectives on telehealth for services to young children with autism. The Promise of New Technologies in an Age of New Health Challenges, 63-73.

Ingersoll, B., \& Berger, N. I. (2015). Parent Engagement With a Telehealth-Based Parent-Mediated Intervention Program for Children With Autism Spectrum Disorders: Predictors of Program Use and Parent Outcomes. Journal Medical Internet Research, 17(10), 1-15.

Ingersoll, B., \& Wainer, A. (2013). Initial efficacy of Project ImPACT: A parentmediated social communication intervention for young children with ASD. Journal of Autism and Developmental Disorders, 43(12), 2943-2952. 
Ingersoll, B., Wainer, A. L., Berger, N. I., Pickard, K. E., \& Bonter, N. (2016). Comparison of a self-directed and therapist-assisted telehealth parent-mediated intervention for children with ASD: A pilot RCT. Journal of Autism and Developmental disorders, 46(7), 2275-2284.

Inzucchi, S. E., Bergenstal, R. M., Buse, J. B., Diamant, M., Ferrannini, E., Nauck, M., . . . Matthews, D. R. (2012). Management of hyperglycemia in type 2 diabetes: a patient-centered approach: position statement of the American Diabetes Association (ADA) and the European Association for the Study of Diabetes (EASD). Diabetes Spectrum, 25(3), 154-171.

Jakobsen, J. C., Gluud, C., Wetterslev, J., \& Winkel, P. (2017). When and how should multiple imputation be used for handling missing data in randomised clinical trials-a practical guide with flowcharts. BMC Medical Research Methodology, 17(1), 1-10.

Jewer, J. (2018). Patients' intention to use online postings of ED wait times: A modified UTAUT model. International Journal of Medical Informatics, 112, 34-39.

John, S. P. (2013). Influence of computer self-efficacy on information technology adoption. International Journal of Information Technology, 19(1), 1-13.

Juarez, A. P., Weitlauf, A. S., Nicholson, A., Pasternak, A., Broderick, N., Hine, J., . . . Warren, Z. (2018). Early Identification of ASD Through Telemedicine: Potential Value for Underserved Populations. Journal of Autism and Developmental Disorders, 48(8), 2601-2610.

Pallant, J. (2016). SPSS Survival Manual. A step By Step Guide To Data Analysis Using IBM SPSS (6 ed.). London: McGraw-Hill Education. 
Kammerer, F. J., Hammon, M., Schlechtweg, P. M., Uder, M., \& Schwab, S. A. (2015). A web based cross-platform application for teleconsultation in radiology. Journal of Telemedicine and Telecare, 21(6), 355-363.

Kanner, L. (1943). Autistic disturbances of affective contact. Nervous Child, 2(3), 217250.

Katharaki, M. (2006). Editorial-Drivers and Supporting Technology to Move Telemedicine into Mainstream Practice. The Journal on Information Technology in Healthcare, 353-355.

Khan, H., Qurashi, M., \& Hayee, I. (2007). Tele-health. The Modern Face of Healthcare. Islamabad: COMSATS Headquarters.

Kijsanayotin, B., Pannarunothai, S., \& Speedie, S. M. (2009). Factors influencing health information technology adoption in Thailand's community health centers: applying the UTAUT model. International Journal of Medical Informatics, 78(6), 404-416.

Kim, E. h., Gellis, Z. D., Bradway, C. K., \& Kenaley, B. (2018). Depression care services and telehealth technology use for homebound elderly in the United States. Aging \& Mental Health, 1-10. doi:10.1080/13607863.2018.1481925

Klin, A., Klaiman, C., \& Jones, W. (2015). Reducing age of autism diagnosis: developmental social neuroscience meets public health challenge. Revue Neurologique, 60, 1-14.

Koch, S. (2006). Home telehealth—current state and future trends. International Journal of Medical Informatics, 75(8), 565-576.

Kohler, F. W. (1999). Examining the services received by young children with autism and their families: A survey of parent responses. Focus on Autism and Other Developmental Disabilities, 14(3), 150-158. 
Koivumäki, T., Ristola, A., \& Kesti, M. (2008). The perceptions towards mobile services: an empirical analysis of the role of use facilitators. Personal and Ubiquitous Computing, 12(1), 67-75.

Kontos, E., Blake, K. D., Chou, W.-Y. S., \& Prestin, A. (2014). Predictors of eHealth usage: insights on the digital divide from the Health Information National Trends Survey 2012. Journal Medical Internet Research, 16(7), 1-16.

Kowitlawakul, Y. (2011). The technology acceptance model: predicting nurses' intention to use telemedicine technology (eICU). CIN: Computers, Informatics, Nursing, 29(7), 411-418.

Kruse, C. S., Soma, M., Pulluri, D., Nemali, N. T., \& Brooks, M. (2017). The effectiveness of telemedicine in the management of chronic heart disease-a systematic review. Journal of the Royal Society of Medicine, 8(3), 1-7.

Lai, M.-C., Lombardo, M. V., \& Baron-Cohen, S. (2014). Autism. The Lancet, 383(9920), 896-910. doi:10.1016/S0140-6736(13)61539-1

Lai, M.-C., Lombardo, M. V., Chakrabarti, B., \& Baron-Cohen, S. (2013). Subgrouping the autism "spectrum": reflections on DSM-5. PLoS Biology, 11(4), 1-7.

Langarizadeh, M., Tabatabaei, M. S., Tavakol, K., Naghipour, M., Rostami, A., \& Moghbeli, F. (2017). Telemental health care, an effective alternative to conventional mental care: A systematic review. Acta Informatica Medica, 25(4), 240-246.

Lee, P. A., Greenfield, G., \& Pappas, Y. (2018). The impact of telehealth remote patient monitoring on glycemic control in type 2 diabetes: a systematic review and meta-analysis of systematic reviews of randomised controlled trials. $B M C$ Health Services Research, 18(1), 1-10. 
Lee, S.-J., Choi, M. J., Rho, M. J., Kim, D.-J., \& Choi, I. Y. (2018). Factors Affecting User Acceptance in Overuse of Smartphones in Mobile Health Services: An Empirical Study Testing a Modified Integrated Model in South Korea. Frontiers In Psychiatry, 9, 1-9. doi:10.3389/fpsyt.2018.00658

Leff, P. T., \& Walizer, E. H. (1992). The uncommon wisdom of parents at the moment of diagnosis. Family Systems Medicine, 10(2), 147-168.

Levy, S. E., Giarelli, E., Lee, L.-C., Schieve, L. A., Kirby, R. S., Cunniff, C., . . Rice, C. E. (2010). Autism spectrum disorder and co-occurring developmental, psychiatric, and medical conditions among children in multiple populations of the United States. Journal of Developmental \& Behavioural Pediatrics, 31(4), 267-275.

Li, J., Talaei-Khoei, A., Seale, H., Ray, P., \& MacIntyre, C. R. (2013). Health care provider adoption of eHealth: systematic literature review. Interactive Journal of Medical Research, 2(1), 1-19.

Lin, B.-S., Wong, A. M., \& Tseng, K. C. (2016). Community-Based ECG Monitoring System for Patients with Cardiovascular Diseases. Journal of Medical Systems, 40(4), 80. doi:10.1007/s10916-016-0442-4

Lindgren, S., Wacker, D., Suess, A., Schieltz, K., Pelzel, K., Kopelman, T., . . . Waldron, D. (2016). Telehealth and autism: Treating challenging behaviour at lower cost. Pediatrics, 137, 167-175.

Lord, C., Risi, S., Lambrecht, L., Cook, E. H., Jr., Leventhal, B. L., DiLavore, P. C., . . . Rutter, M. (2000). The autism diagnostic observation schedule-generic: a standard measure of social and communication deficits associated with the spectrum of autism. Journal of Autism and Developmental Disorders, 30(3), 205-223. 
Lord, C., Rutter, M., DiLavore, P., Risi, S., Gotham, K., \& Bishop, S. (2012). Autism Diagnostic Observation Schedule Second Edition. Torrance, CA: Western Psychological Services.

Lord, C., Rutter, M., \& Le Couteur, A. (1994). Autism Diagnostic Interview-Revised: a revised version of a diagnostic interview for caregivers of individuals with possible pervasive developmental disorders. Journal of Autism and Developmental Disorders, 24(5), 659-685.

Lovell, N. H., Redmond, S. J., Basilakis, J., Shany, T., \& Celler, B. G. (2010). Telehealth technologies for managing chronic disease-experiences from Australia and the UK. Proceedings of the Annual International Conference of the IEEE Engineering in Medicine and Biology, Aregntina, 5267-5269. doi:10.1109/IEMBS.2010.5626312

Macdonald, E. M., Perrin, B. M., Hyett, N., \& Kingsley, M. I. (2019). Factors influencing behavioural intention to use a smart shoe insole in regionally based adults with diabetes: a mixed methods study. Journal of Foot and Ankle Research, 12(1), 1-9.

Machalicek, W., Lequia, J., Pinkelman, S., Knowles, C., Raulston, T., Davis, T., \& Alresheed, F. (2016). Behavioural telehealth consultation with families of children with autism spectrum disorder. Behavioural Interventions, 31(3), 223250.

Maenner, M. J., Shaw, K. A., \& Baio, J. (2020). Prevalence of autism spectrum disorder among children aged 8 years — autism and developmental disabilities monitoring network, 11 sites, United States, 2016. MMWR Surveillance Summaries, 69(4), 1-12. 
Maheu, M., Whitten, P., \& Allen, A. (2001). E-health, telehealth, and telemedicine: A guide to start-up and success. San Francisco: Jossey-Bass.

Mandell, D. S., Novak, M. M., \& Zubritsky, C. D. (2005). Factors associated with age of diagnosis among children with autism spectrum disorders. Pediatrics, 116(6), 1480-1486.

Mansell, W., \& Morris, K. (2004). A survey of parents' reactions to the diagnosis of an autistic spectrum disorder by a local service: Access to information and use of services. Autism, 8(4), 387-407.

Matson, J. L. (2008). Clinical assessment and intervention for autism spectrum disorders ( $1^{\text {st }}$ ed.). Amsterdam: Elsevier.

McConkey, R., Truesdale-Kennedy, M., \& Cassidy, A. (2009). Mothers' recollections of early features of autism spectrum disorders. Child and Adolescent Mental Health, 14(1), 31-36.

McCrossan, B. A., Grant, B., Morgan, G. J., Sands, A. J., Craig, B., \& Casey, F. A. (2008). Diagnosis of congenital heart disease in neonates by videoconferencing: an eight-year experience. Journal of Telemedicine and Telecare, 14(3), 137-140.

McEachin, J. J., Smith, T., \& Ivar Lovaas, O. (1993). Long-term outcome for children with autism who received early intensive behavioural treatment. American Journal of Mental Retardation, 97, 359-359.

McLeod, B. D., Jensen-Doss, A., \& Ollendick, T. H. (2013). Diagnostic and behavioural assessment in children and adolescents: A clinical guide. New York: Guilford Press.

McMorris, C. A., Cox, E., Hudson, M., Liu, X., \& Bebko, J. M. (2013). The diagnostic process of children with autism spectrum disorder: Implications for early 
identification and intervention. Journal on Developmental Disabilities, 19(2), $42-49$.

Meadan, H., \& Daczewitz, M. E. (2015). Internet-based intervention training for parents of young children with disabilities: a promising service-delivery model. Early Child Development and Care, 185(1), 155-169.

Miller, E. A. (2007). Solving the disjuncture between research and practice: telehealth trends in the 21st century. Health Policy, 82(2), 133-141.

Moh, T. A., \& Magiati, I. (2012). Factors associated with parental stress and satisfaction during the process of diagnosis of children with autism spectrum disorders. Research in Autism Spectrum Disorders, 6(1), 293-303.

Momani, A. M., Jamous, M. M., \& Hilles, S. M. (2017). Technology Acceptance Theories: Review and Classification. International Journal of Cyber Behaviour, Psychology and Learning (IJCBPL), 7(2), 1-14.

Montani, S., Bellazzi, R., Quaglini, S., \& D'Annunzio, G. (2001). Meta-analysis of the effect of the use of computer-based systems on the metabolic control of patients with diabetes mellitus. Diabetes Technology \& Therapeutics, 3(3), 347-356.

Moore, G. C., \& Benbasat, I. (1991). Development of an instrument to measure the perceptions of adopting an information technology innovation. Information Systems Research, 2(3), 192-222.

Morse, M. J. (2003). Principles of mixed methods and multi-method research design. In A. Tashakkori \& C. Teddlie (Eds.), Handbook of mixed methods in social and behavioural research (pp. 189-208). Thousand Oaks: SAGE.

Munn, Z., Peters, M. D., Stern, C., Tufanaru, C., McArthur, A., \& Aromataris, E. (2018). Systematic review or scoping review? Guidance for authors when 
choosing between a systematic or scoping review approach. BMC Medical Research Methodology, 18(1), 143.

Nachmias, C. F., \& Nachmias, D. (1996). Research Methods in the Social Sciences (5 $5^{\text {th }}$ ed.). London: Arnold Publication Inc.

National Autistic Society. (2017a). Autism facts and history. Retrieved April 3, 2018, from https://www.autism.org.uk/007874

National Autistic Society. (2017b). Sevices and support in Wales. Retrieved from https://www.autism.org.uk/directory

National Autistic Society. (2018a). What is autism? Retrieved from

\section{https://www.autism.org.uk/advice-and-guidance/what-is-autism}

National Autistic Society. (2018b). Diagnosis. Retrieved from

https://www.autism.org.uk/advice-and-guidance/topics/diagnosis

National Health Service. (2019). Getting diagnosed. Retrieved from

https://www.nhs.uk/conditions/autism/getting-diagnosed/

National Institute for Health and Care Excellence. (2005). Who we are. Retrieved from

\section{https://www.nice.org.uk/about/who-we-are}

National Institute for Health and Care Excellence. (2017). Autism spectrum disorder in under 19s: recognition, referral and diagnosis. Retrieved from https://www.nice.org.uk/guidance/cg128

Nazneen, N., Matthews, N., Smith, C. J., Rozga, A., Abowd, G. D., Oberleitner, R., . .. Arriaga, R. I. (2015). Use of a novel imaging technology for remote autism diagnosis: a reflection on experience of stakeholders. Procedia Manufacturing, 3, 293-300.

Nazneen, N., Oberleitner, R., \& Reischl, U. (2016). Asynchronous Telemedicine System for the Remote Diagnosis of ASDTelemedicine (pp. 1-14): SMGroup. 
Retrieved from http://www.smgebooks.com/telemedicine/chapters/TELMED16-02.pdf.

Nazneen, N., Rozga, A., Smith, C., Oberleitner, R., Abowd, G., \& Arriaga, R. (2015). A novel system for supporting autism diagnosis using home videos: iterative development and evaluation of system design. JMIR Mhealth Uhealth, 3(2).

NHLBI. (n.d.). Study Quality Assessment Tools. Retrieved from https://www.nhlbi.nih.gov/health-topics/study-quality-assessment-tools

Norris, A. C. (2002). Essentials of telemedicine and telecare. West Sussex, England: J. Wiley \& Sons.

Norton, K., Longley, M., \& Ponton, M. (2012). The best configuration of hospital services for wales: A review of the evidence. Retrieved from http://www.wales.nhs.uk/sitesplus/documents/902/ACCESS FINAL.pdf

O'Cathain, A., \& Thomas, K. J. (2004). " Any other comments?" Open questions on questionnaires-a bane or a bonus to research? BMC Medical Research Methodology, 4(1), 25.

Oberleitner, R., Elison-Bowers, P., Reischl, U., \& Ball, J. (2007). Optimizing the personal health record with special video capture for the treatment of autism. Journal of Developmental and Physical Disabilities, 19(5), 513-518.

Office for National Statistics. (2016). Wales. Retrieved from https://www.ons.gov.uk/methodology/geography/ukgeographies/administrati vegeography/wales

Oliver, C., Petty, J., Ruddick, L., \& Bacarese-Hamilton, M. (2012). The association between repetitive, self-injurious and aggressive behaviour in children with severe intellectual disability. Journal of Autism and Developmental Disorders, 42(6), 910-919. 
Ooi, K. L., Ong, Y. S., Jacob, S. A., \& Khan, T. M. (2016). A meta-synthesis on parenting a child with autism. Neuropsychiatric Disease and Treatment, 12, $745-762$.

Opoku, M. O., \& Francis, E.-K. (2020). Relevance of the technology acceptance model (TAM) in information management research: A Review of selected empirical evidence. Research Journal of Business and Management, 7(1), 34-44.

Or, C. K., \& Karsh, B.-T. (2009). A systematic review of patient acceptance of consumer health information technology. Journal of the American Medical Informatics Association, 16(4), 550-560.

Ortiz, E., \& Clancy, C. M. (2003). Use of information technology to improve the quality of health care in the United States. Health Services Research, 38(2), 112. doi:10.1111/1475-6773.00127

Osborne, L. A., \& Reed, P. (2008). Parents' perceptions of communication with professionals during the diagnosis of autism. Autism, 12(3), 309-324.

Oshlyansky, L., Cairns, P., \& Thimbleby, H. (2007). Validating the Unified Theory of Acceptance and Use of Technology (UTAUT) tool cross-culturally. Paper presented at the Proceedings of the 21st BCS HCI Group Conference, 2, 8386.

Osterling, J. A., Dawson, G., \& Munson, J. A. (2002). Early recognition of 1-year-old infants with autism spectrum disorder versus mental retardation. Development and Psychopathology, 14(2), 239-251.

Parmanto, B., Pulantara, I. W., Schutte, J. L., Saptono, A., \& McCue, M. P. (2013). An integrated telehealth system for remote administration of an adult autism assessment. Telemedicine and e-Health, 19(2), 88-94. doi:10.1089/tmj.2012.0104 
Peat, J. K. (2002). Health science research. London: SAGE Publications, Ltd

Pickard, K. E., Wainer, A. L., Bailey, K. M., \& Ingersoll, B. R. (2016). A mixedmethod evaluation of the feasibility and acceptability of a telehealth-based parent-mediated intervention for children with autism spectrum disorder. Autism, 20(7), 845-855.

Potter, C. A. (2017). "I received a leaflet and that is all": Father experiences of a diagnosis of autism. British Journal of Learning Disabilities, 45(2), 95-105.

Quaosar, G. A. A., Hoque, M. R., \& Bao, Y. (2018). Investigating factors affecting Elderly's intention to use m-health services: An empirical study. Telemedicine and e-Health, 24(4), 309-314.

Randall, M., Egberts, K. J., Samtani, A., Scholten, R. J., Hooft, L., Livingstone, N., . . . Williams, K. (2018). Diagnostic tests for autism spectrum disorder (ASD) in preschool children. Cochrane Database of Systematic Reviews(7), 1-102.

Rattazzi, A. (2014). The importance of early detection and early intervention for children with autism spectrum conditions. Vertex (Buenos Aires, Argentina), 25(116), 290-294.

Rawstorne, P., Jayasuriya, R., \& Caputi, P. (2000). Issues in predicting and explaining usage behaviours with the technology acceptance model and the theory of planned behaviour when usage is mandatory. Proceedings of the twenty first International Conference on Information Systems, 5, 35-44.

Reed, P., \& Osborne, L. A. (2012). Diagnostic practice and its impacts on parental health and child behaviour problems in autism spectrum disorders. Archives of Disease in Childhood, 97(10), 927-931.

Reese, R., Jamison, T., Braun, M., Wendland, M., Black, W., Hadorn, M., . . Prather, C. (2015). Brief Report: Use of Interactive Television in Identifying Autism in 
Young Children: Methodology and Preliminary Data. Journal of Autism \& Developmental Disorders, 45(5), 1474-1482. doi:10.1007/s10803-014-2269-5

Reese, R. M., Braun, M. J., Hoffmeier, S., Stickle, L., Rinner, L., Smith, C., . . Jarrett, L. (2015). Preliminary Evidence for the Integrated Systems Using Telemedicine. Telemedicine and e-Health, 21(7), 581-587.

Reese, R. M., Jamison, R., Wendland, M., Fleming, K., Braun, M. J., Schuttler, J. O., \& Turek, J. (2013). Evaluating interactive videoconferencing for assessing symptoms of autism. Telemedicine Journal And E-Health: The Official Journal Of The American Telemedicine Association, 19(9), 671-677. doi:10.1089/tmj.2012.0312

Rho, M. J., Kim, H. S., Chung, K., \& Choi, I. Y. (2015). Factors influencing the acceptance of telemedicine for diabetes management. Cluster Computing, 18(1), 321-331.

Rogers, E. (1995). Diffusion of Innovations (4 ${ }^{\text {th }}$ ed.). New York: Free Press.

Rogers, E. (2003). Diffusion of Innovations (5 ${ }^{\text {th }}$ ed.). New York: Free Press.

Rousseeuw, P. J., \& Hubert, M. (2018). Anomaly detection by robust statistics. Wiley Interdisciplinary Reviews: Data Mining and Knowledge Discovery, 8(2), 1-14.

Ruble, L. A., Heflinger, C. A., Renfrew, J. W., \& Saunders, R. C. (2005). Access and service use by children with autism spectrum disorders in Medicaid managed care. Journal of Autism and Developmental Disorders, 35(1), 3-13.

Ryu, M.-H., Kim, S., \& Lee, E. (2009). Understanding the factors affecting online elderly user's participation in video UCC services. Computers in Human Behaviour, 25(3), 619-632.

Salomone, E., Charman, T., McConachie, H., \& Warreyn, P. (2016). Child's verbal ability and gender are associated with age at diagnosis in a sample of young 
children with ASD in Europe. Child: Care, Health and Development, 42(1), 141-145.

Sarantakos, S. (2012). Social research (4th ed.). London: Red Globe Press.

Schutte, J. L., McCue, M. P., Parmanto, B., McGonigle, J., Handen, B., Lewis, A., . . . Saptono, A. (2015). Usability and reliability of a remotely administered adult autism assessment, the autism diagnostic observation schedule (ADOS) module 4. Telemedicine and e-Health, 21(3), 176-184.

Sheppard, B. H., Hartwick, J., \& Warshaw, P. R. (1988). The theory of reasoned action: A meta-analysis of past research with recommendations for modifications and future research. Journal of Consumer Research, 15(3), 325343.

Shores, M. M., Ryan-Dykes, P., Williams, R. M., Mamerto, B., Sadak, T., Pascualy, M., . . Peskind, E. R. (2004). Identifying undiagnosed dementia in residential care veterans: comparing telemedicine to in-person clinical examination. International Journal of Geriatric Psychiatry, 19(2), 101-108. doi:10.1002/gps.1029

Siegel, B. (2008). Getting the best for your child with autism: An expert's guide to treatment ( $1^{\text {st }}$ ed.). New York: The Guilford Press.

Siklos, S., \& Kerns, K. A. (2006). Assessing need for social support in parents of children with autism and Down syndrome. Research in Developmental Disabilities, 36(7), 921-933.

Siklos, S., \& Kerns, K. A. (2007). Assessing the diagnostic experiences of a small sample of parents of children with autism spectrum disorders. Research in Developmental Disabilities, 28(1), 9-22. 
Simacek, J., Dimian, A. F., \& McComas, J. J. (2017). Communication intervention for young children with severe neurodevelopmental disabilities via telehealth. Journal of Autism and Developmental Disorders, 47(3), 744-767.

Simonoff, E., Pickles, A., Charman, T., Chandler, S., Loucas, T., \& Baird, G. (2008). Psychiatric disorders in children with autism spectrum disorders: prevalence, comorbidity, and associated factors in a population-derived sample. Journal of the American Academy of Child \& Adolescent Psychiatry, 47(8), 921-929.

Smith, C. J., Rozga, A., Matthews, N., Oberleitner, R., Nazneen, N., \& Abowd, G. (2017). Investigating the accuracy of a novel telehealth diagnostic approach for autism spectrum disorder. Psychological Assessment, 29(3), 245-252.

Sobowale, K., Nguyen, M., Weiss, B., Van, T. H., \& Trung, L. (2016). Acceptability of internet interventions for youth mental health in Vietnam. Global Mental Health, 3, 1-9.

Soke, G., Maenner, M., Christensen, D., Kurzius-Spencer, M., \& Schieve, L. (2018). Prevalence of co-occurring medical and behavioural conditions/symptoms among 4-and 8-year-old children with autism spectrum disorder in selected areas of the United States in 2010. Journal of Autism and Developmental Disorders, 48(8), 2663-2676.

Sood, S., Mbarika, V., Jugoo, S., Dookhy, R., Doarn, C. R., Prakash, N., \& Merrell, R. C. (2007). What is telemedicine? A collection of 104 peer-reviewed perspectives and theoretical underpinnings. Telemedicine Journal and eHealth, 13(5), 573-590. doi:10.1089/tmj.2006.0073

Stainbrook, J. A., Weitlauf, A. S., Juarez, A. P., Taylor, J. L., Hine, J., Broderick, N., . . . Warren, Z. (2018). Measuring the service system impact of a novel 
telediagnostic service program for young children with autism spectrum disorder. Autism, 1-6. doi:10.1177/1362361318787797

Sterne, J. A., White, I. R., Carlin, J. B., Spratt, M., Royston, P., Kenward, M. G., . . Carpenter, J. R. (2009). Multiple imputation for missing data in epidemiological and clinical research: Potential and pitfalls. British Medical Journal, 338, b2393. doi:10.1136/bmj.b2393

Steventon, A., Bardsley, M., Doll, H., Tuckey, E., \& Newman, S. P. (2014). Effect of telehealth on glycaemic control: analysis of patients with type 2 diabetes in the Whole Systems Demonstrator cluster randomised trial. BMC Health Services Research, 14(1), 1-12.

Stoltzfus, J. C. (2011). Logistic regression: a brief primer. Academic Emergency Medicine, 18(10), 1099-1104.

Stone, M. A., Charpentier, G., Doggen, K., Kuss, O., Lindblad, U., Kellner, C., . . . Trento, M. (2013). Quality of care of people with type 2 diabetes in eight European countries: findings from the Guideline Adherence to Enhance Care (GUIDANCE) study. Diabetes Care, 36(9), 2628-2638.

Straub, E. T. (2009). Understanding technology adoption: Theory and future directions for informal learning. Review of Educational Research, 79(2), 625-649.

Sue, V. M., \& Ritter, L. A. (2012). Conducting online surveys (2nd ed.). Los Angeles: Sage.

Suess, A. N., Romani, P. W., Wacker, D. P., Dyson, S. M., Kuhle, J. L., Lee, J. F., . . . Waldron, D. B. (2014). Evaluating the treatment fidelity of parents who conduct in-home functional communication training with coaching via telehealth. Journal of Behavioural Education, 23(1), 34-59. doi:10.1007/s10864-013-9183-3 
Suess, A. N., Wacker, D. P., Schwartz, J. E., Lustig, N., \& Detrick, J. (2016). Preliminary evidence on the use of telehealth in an outpatient behaviour clinic. Journal of Applied Behaviour Analysis, 49(3), 686-692. doi:10.1002/jaba.305

Taherdoost, H. (2018). A review of technology acceptance and adoption models and theories. Procedia Manufacturing, 22, 960-967.

Tang, D., \& Chen, L. (2011). A review of the evolution of research on information Technology Acceptance Model. Proceedings of the 2011 International Conference on Business Management and Electronic Information, 2, 588591. doi:10.1109/ICBMEI.2011.5917980

Taylor, S., \& Todd, P. A. (1995). Understanding information technology usage: A test of competing models. Information Systems Research, 6(2), 144-176.

The Welsh NHS Confederation. (2018). Rural Health and Care Services in Wales. Retrieved from https://www.nhsconfed.org//media/Confederation/Files/Wales-Confed/Rural-Health-and-Care-Servicesin-Wales.pdf

Thomas, K. C., Ellis, A. R., McLaurin, C., Daniels, J., \& Morrissey, J. P. (2007). Access to care for autism-related services. Journal of Autism and Developmental Disorders, 37(10), 1902-1912.

Thompson, R. L., Higgins, C. A., \& Howell, J. M. (1991). Personal computing: toward a conceptual model of utilization. Management Information Systems Quarterly, 15, 125-143.

Tiger, J. H., Hanley, G. P., \& Bruzek, J. (2008). Functional communication training: A review and practical guide. Behaviour Analysis in Practice, 1(1), 16-23. 
Tricco, A. C., Lillie, E., Zarin, W., O'Brien, K. K., Colquhoun, H., Levac, D., . . . Weeks, L. (2018). PRISMA extension for scoping reviews (PRISMA-ScR): checklist and explanation. Annals of Internal Medicine, 169(7), 467-473.

Trott, P., \& Blignault, I. (1998). Cost evaluation of a telepsychiatry service in northern Queensland. Journal of Telemedicine and Telecare, 4, 66-68.

Turner, M., Kitchenham, B., Brereton, P., Charters, S., \& Budgen, D. (2010). Does the technology acceptance model predict actual use? A systematic literature review. Information and Software Technology, 52(5), 463-479.

United Psychiatric Association. (1980). Diagnostic and Statistical Manual of Mental Disorders iii. Washington, DC: American Psychiatric Association.

Van der Vaart, R., Atema, V., \& Evers, A. W. M. (2016). Guided online selfmanagement interventions in primary care: a survey on use, facilitators, and barriers. BMC Family Practice, 17, 1-9. doi:10.1186/s12875-016-0424-0

Van Houwelingen, C. T., Ettema, R. G., Antonietti, M. G., \& Kort, H. S. (2018). Understanding Older People's Readiness for Receiving Telehealth: MixedMethod Study. Journal Medical Internet Research, 20(4), 1-16. doi:10.2196/jmir.8407

Venkatesh, V., \& Bala, H. (2008). Technology acceptance model 3 and a research agenda on interventions. Decision Sciences, 39(2), 273-315.

Venkatesh, V., \& Davis, F. D. (2000). A theoretical extension of the technology acceptance model: Four longitudinal field studies. Management Science, 46(2), 186-204.

Venkatesh, V., Morris, M. G., Davis, G. B., \& Davis, F. D. (2003). User acceptance of information technology: Toward a unified view. MIS Quarterly, 425-478. 
Vismara, L. A., McCormick, C., Young, G. S., Nadhan, A., \& Monlux, K. (2013). Preliminary findings of a telehealth approach to parent training in autism. Journal of autism and developmental disorders, 43(12), 2953-2969. doi:10.1007/s10803-013-1841-8

Vismara, L. A., Young, G. S., \& Rogers, S. J. (2012). Telehealth for expanding the reach of early autism training to parents. Autism Research and Treatment, 2012, 1-12.

Vohra, R., Madhavan, S., Sambamoorthi, U., \& St Peter, C. (2014). Access to services, quality of care, and family impact for children with autism, other developmental disabilities, and other mental health conditions. Autism, 18(7), 815-826.

Wacker, D. P., Berg, W. K., Harding, J. W., Derby, K. M., Asmus, J. M., \& Healy, A. (1998). Evaluation and long-term treatment of aberrant behaviour displayed by young children with disabilities. Journal of Developmental and Behavioural Pediatrics, 19(4), 260-266.

Wacker, D. P., Lee, J. F., Dalmau, Y. C. P., Kopelman, T. G., Lindgren, S. D., Kuhle, J., Pelzel, K.E., Dyson, S. . . Waldron, D. B. (2013). Conducting functional communication training via telehealth to reduce the problem behaviour of young children with autism. Journal of Developmental and Physical Disabilities, 25(1), 35-48.

Wacker, D. P., Lee, J. F., Dalmau, Y. C. P., Kopelman, T. G., Lindgren, S. D., Kuhle, J., Pelzel, K. E. \& Waldron, D. B. (2013). Conducting functional analyses of problem behaviour via telehealth. Journal of Applied Behaviour Analysis, 46(1), 31-46. 
Wagner, N., Hassanein, K., \& Head, M. (2010). Computer use by older adults: A multi-disciplinary review. Computers in Human Behaviour, 26(5), 870-882.

Wainer, A. L., \& Ingersoll, B. R. (2015). Increasing access to an ASD imitation intervention via a telehealth parent training program. Journal of Autism and Developmental Disorders, 45(12), 3877-3890.

Walliman, N. (2006). Social Research Methods. London: SAGE Publications.

Waterhouse, L. (2013). Rethinking Autism Variation and Complexity ( $1^{\text {st }}$ ed.). London: ELSEVIER.

Welsh Government. (2015). Informed health and care - A digital health and social care strategy for Wales. Retrieved from https://gov.wales/sites/default/files/publications/2019-03/informed-healthand-care-a-digital-health-and-social-care-strategy-for-wales.pdf

Welsh Government. (2016). Refreshed Autistic Strategic Action plan 2016 for Wales. Retrieved from https://gov.wales/sites/default/files/publications/201903/refreshed-autistic-spectrum-disorder-strategic-action-plan.pdf

Welsh Government. (2017). Prosperity for All: The National Strategy: Taking Wales Forward. Retrieved from https://gov.wales/sites/default/files/publications/2017-10/prosperity-for-allthe-national-strategy.pdf

Welsh Government. (2018). A Healthier Wales: Our Plan for Health and Social Care. Retrieved from https://www.wwcp.org.uk/wp-content/uploads/2018/07/1807-13-ITEM-5-A-Healthier-Wales-Summary-June-2018.pdf

Welsh Government. (2019). National Survey for Wales, 2018-19 Internet use and digital skills. Retrieved from https://gov.wales/sites/default/files/statistics- 
and-research/2019-09/internet-use-and-digital-skills-national-survey-walesapril-2018-march-2019-207.pdf

Welsh Government. (2020). Statistical First Release. Retrieved from https://gov.wales/sites/default/files/statistics-and-research/2020-05/summarystatistics-regions-wales-2020-629.pdf

Wetherby, A. M., \& Woods, J. J. (2006). Early social interaction project for children with autism spectrum disorders beginning in the second year of life: A preliminary study. Topics in Early Childhood Special Education, 26(2), 67-82.

Wiggins, L. D., Baio, J., \& Rice, C. (2006). Examination of the time between first evaluation and first autism spectrum diagnosis in a population-based sample. Journal of Developmental \& Behavioural Pediatrics, 27(2), S79-S87.

Williams, M. D., Rana, N. P., \& Dwivedi, Y. K. (2015). The unified theory of acceptance and use of technology (UTAUT): a literature review. Journal of Enterprise Information Management, 28(3), 443-488.

Wilson, N., \& McLean, S. (1994 ). Questionnaire Design: A Practical Introduction. Newtownabbey: University of Ulster Press.

Wong, V. C., \& Kwan, Q. K. (2010). Randomized controlled trial for early intervention for autism: a pilot study of the Autism 1-2-3 Project. Journal of Autism and Developmental Disorders, 40(6), 677-688.

Woolley, H., Stein, A., Forrest, G., \& Baum, J. (1989). Imparting the diagnosis of life threatening illness in children. British Medical Journal, 298(6688), 1623-1626.

World Health Organisation. (2018). ICD-11. Retrieved from https://www.who.int/classifications/icd/en/

World Health Organisation. (2019). Autism spectrum disorders. Retrieved from https://www.who.int/news-room/fact-sheets/detail/autism-spectrum-disorders 
World Health Organisation. (1993). The ICD-10 classification of mental and behavioural disorders: Diagnostic criteria for research. Geneva: World Health Organisation.

World Health Organisation. (2010). Telemedicine: opportunities and developments in Member States: report on the second global survey on eHealth. Retrieved from https://www.who.int/goe/publications/goe_telemedicine_2010.pdf

WorldAtlas. (2020). Wales. Retrieved from

https://www.worldatlas.com/webimage/countrys/europe/wales/ukwlandst.htm Zagzoug, M. S. (2015). Exploring Acceptance of Using an Online Platform to Teach Parents of Children with Autism Methods in Applied Behaviour Analysis (ABA). (Doctoral dissertation). Retrieved from Seton Hall University Dissertations and Theses (ETDs).

Zhang H, Cocosila M, Archer N. (2010). Factors of adoption of mobile information technology by homecare nurses: a technology acceptance model 2 approach. Computers Informatics Nursing, 28(1), 49-56.

Zhang, X., Yu, P., Yan, J., Hu, H., \& Goureia, N. (2012). Patients' perceptions of web self-service applications in primary healthcare. Studies in Health Technology \& Informatics, 242-249. , 46(5), 1725-1736.

Ziats, M. N., \& Rennert, O. M. (2016). The evolving diagnostic and genetic landscapes of autism spectrum disorder. Frontiers in Genetics, 7, 1-6.

Zwaigenbaum, L., Bauman, M. L., Choueiri, R., Kasari, C., Carter, A., Granpeesheh, D., . . Fein, D. (2015). Early intervention for children with autism spectrum disorder under 3 years of age: recommendations for practice and research. Pediatrics, 136, 60-81. 
Zwaigenbaum, L., Nicholas, D. B., Muskat, B., Kilmer, C., Newton, A. S., Craig, W. R., . . Roberts, W. (2016). Perspectives of Health Care Providers Regarding Emergency Department Care of Children and Youth with Autism Spectrum Disorder. Journal of Autism and Developmental Disorders, 46(5), 1725-1736. 